\title{
Prediction of the effects of compositional mixing in a reservoir on conversion to natural gas storage
}

\author{
Alan Wallace Brannon \\ West Virginia University
}

Follow this and additional works at: https://researchrepository.wvu.edu/etd

\section{Recommended Citation}

Brannon, Alan Wallace, "Prediction of the effects of compositional mixing in a reservoir on conversion to natural gas storage" (2011). Graduate Theses, Dissertations, and Problem Reports. 3032.

https://researchrepository.wvu.edu/etd/3032

This Dissertation is protected by copyright and/or related rights. It has been brought to you by the The Research Repository @ WVU with permission from the rights-holder(s). You are free to use this Dissertation in any way that is permitted by the copyright and related rights legislation that applies to your use. For other uses you must obtain permission from the rights-holder(s) directly, unless additional rights are indicated by a Creative Commons license in the record and/ or on the work itself. This Dissertation has been accepted for inclusion in WVU Graduate Theses, Dissertations, and Problem Reports collection by an authorized administrator of The Research Repository @ WVU.

For more information, please contact researchrepository@mail.wvu.edu. 


\author{
Alan Wallace Brannon \\ Dissertation submitted to the \\ College of Engineering and Mineral Resources \\ at West Virginia University \\ in partial fulfillment of the requirements \\ for the degree of
}

Doctor of Philosophy in Petroleum and Natural Gas Engineering

\author{
Khashayar Aminian, Ph.D., Chair \\ Samuel Ameri, M.S. \\ James Ammer, \\ H. Ilkin Bilgesu, Ph.D. \\ Shahab Mohaghegh, Ph.D. \\ Morgantown, West Virginia \\ 2011
}

Keywords: Gas Condensate; Natural Gas storage; Compositional Reservoir Modeling

Copyright 2011 Alan Wallace Brannon 


\section{ABSTRACT \\ Conversion of a Natural Gas Condensate Reservoir to Gas Storage by Alan W. Brannon}

The increased interest in the development of new Gas Storage Fields over the last several decades has created some interesting challenges for the industry. Most existing gas storage fields have been developed from depleted natural gas and oil production reservoirs. It is not uncommon for gas storage fields to be developed in water bearing aquifer zones where the presence of conventional type reservoirs is limited. Natural Gas Condensate reservoirs are typically not found in the eastern United States where most gas storage facilities are developed close to the colder market and increased population centers. Therefore, data is limited on how the lean dry storage gas will interact with the remaining residual gas condensate liquid and wet gas at reservoir pressure and temperature conditions.

When a depleted gas storage reservoir is being evaluated for storage potential many questions arise that if addressed during the depletion phase would help in the storage evaluation process. However, the main focus of the production phase is to maximize production at the lowest possible cost. As a result of this data concerning fluid analysis and production records can be somewhat difficult. Gas Condensate reservoirs contain multi-component hydrocarbon mixtures that require equation of state models to predict phase behavior under differing conditions. Therefore, adequate initial gas and liquid sampling is necessary that can then have laboratory PVT analysis performed to establish fluid properties such as (dew point, z-factor, viscosity, oil relative volume, etc.). Obtaining this data can sometimes be a challenge due to divesture company's poor recordkeeping and not wanting to incur the expense of extended analysis. Also, acquiring accurate production history records on the liquid and gas production volumes can sometimes be difficult to obtain and assimilate. These are just a few of the problems that can surface when a storage company wants to study the conversion process from a gas condensate reservoir to a viable storage project.

Another objective of this study and research is to look at some of the development challenges in the conversion of a gas condensate reservoir to gas storage and how these challenges affects the overall design in storage facilities.

In this study compositional reservoir simulators coupled with equation of state models was used to evaluate a natural gas condensate reservoir under storage development and operating conditions. The Equation of State (EOS) models was used to perform initial fluid analysis and compare to what laboratory data is available to initialize the reservoir fluid set in the model. The compositional reservoir simulator was then built utilizing a certain geologic characterization for the reservoir properties. The phase behavior model can then be used along with the reservoir simulator to validate the primary production history matching and then perform storage scenario analysis. 
After an accurate compositional reservoir model was developed and the model has validated the original production history, the storage scenarios were simulated to look at how well configurations and rate profiles affect mixing and the impact on condensate yields.

Since this project will be in service and operating, the actual storage operating data can be integrated into the reservoir design model to further enhance the predictive capabilities of the reservoir model. It is estimated that full development will take several years allowing the model to be calibrated and improved over time.

When a gas storage field is developed, typically there is a need to drill more active injection and withdrawal wells to meet the deliverability design parameter. When a field is discovered there is no immediate need to drill excess wells into the field when for production purposes a few wells will suffice. However, when a gas storage field is being developed depending the areal extent and volume of the reservoir a significant number of new wells may be required to meet the deliverability design. When the wells are designed they can be completed in three ways: vertically, directional and horizontally. Each completion has its pro and cons that need to be considered and with a condensate depleted reservoir more emphasis needs to be placed on how the individual well completions will affect field deliverability performance and residual condensate recovery.

Another consideration in the development of storage is an accurate prediction of the condensate yield rates and the ultimate condensate recovery under gas storage cycling operations. This is important due to the requirement to build adequate surface facilities and project the anticipated yearly condensate production. After a reliable composition reservoir simulator was built the reservoir properties were adjusted to evaluate the impact the storage scenarios have on mixing and condensate recovery. With the calibrated model the effects of resident time on fluid diffusion as a result of mixing were evaluated.

The reservoir simulator was able to predict the impact of surface facilities on condensate recovery and gas deliverability. Condensate blockage around the wellbore is a common problem with mature condensate fields. The wellbore representations in the model were evaluated for the effects of this problem on storage operations.

The reservoir simulator was also used to optimize the number of wells needed to achieve the desired storage field design requirements. The simulator was used to estimate the number of years that condensate production will be a limiting factor in reaching $100 \%$ storage field development. 


\section{ACKNOWLEDGMENTS}

The author would like to first thank god for giving me the strength to complete my research and doctoral work.

I sincerely appreciate Dr. Khashayar Aminian, my graduate advisor and Doctoral committee chairman who without his patience and support this body of work would not have been possible.

Words cannot express the gratitude I have for Chairman Sam Ameri. I have been a student, colleague and most important friend to Mr. Ameri for over thirty years and will always remember him saying you must complete this advanced degree. His support and advice serving on my committee has been invaluable.

I would like to express my appreciation to all of my committee members for taking the time from their busy schedules and lives to work with me to complete this dissertation work.

Also, I would especially like to thank Dominion Transmission Inc. for allowing me the opportunity to complete this work.

It is my hope that my dedication and perseverance in working to obtain this Doctoral Degree can someday inspire or provide a seed for my loving children (Alexis, Jacob and Lacey) to work hard and strive to be best at whatever they choose to do in life.

Most importantly, I would like to thank my wife Barbara for without her support and patience this long endeavor would not have been possible to complete. 


\section{TABLE OF CONTENTS}

LIST OF TABLES

LIST OF FIGURES

ABSTRACT

CHAPTER 1 INTRODUCTION

Introductory Comments 1

Research Goal $\quad 2$

Problem Statement 3

Objectives $\quad 4$

Background $\quad 5-7$

Gas Storage $\quad 7-11$

CHAPTER 2 LITERATURE REVIEW

2.1 Gas cycling/modeling literature $\quad 12-16$

2.2 EOS/Fluid Characterization/Sampling literature 16-23

CHAPTER 3 METHODOLOGY AND OBJECTIVES

Discussion $\quad 25$

$\begin{array}{lr}\text { Comparison with Laboratory PVT Study } & \text { 26-27 }\end{array}$

Comparison with Primary Production History $\quad 27$

Material Balance Study $\quad 27$

Simulation of Storage $\quad 28$

Compositional Model Development 29-30

Reservoir Fluid Phase Behavior Prediction $\quad 31$

Simulation of Primary Production $\quad$ 32-35

Simulation of Storage Performance with Vertical Wells $\quad 35$

Simulation of Storage Performance with Horizontal Wells 36

Storage Operational Design Evaluation 36-37

Reservoir and Fluid Properties Evaluation 37-38

\section{CHAPTER 4 RESERVOIR MODEL DEVELOPMENT}

Reservoir History

$39-42$

Geologic Description $\quad 43$

Early Seismic Work $\quad$ 44-51

Open Hole Log Analysis $\quad$ 52-59

Compositional Reservoir Models $\quad$ 60-61

Reservoir and EOS Model Selection $\quad$ 62-63

Reservoir Model Development $\quad 63$ 
Fluid Model Characterization $\quad 64$

Initial Reservoir Fluid Evaluation (PR-EOS) 64-67

Comparison with Laboratory PVT Study 68-71

Comparison with Primary Liquids Production History $\quad 72-73$

Initial Fluid Evaluation (WinProp) $\quad 73-80$

Material Balance Analysis $\quad$ 80-82

Simulation of Storage Gas Cycling 82-83

Compositional Model Development 84-91

History Matching Discussion $\quad$ 92-95

Simulation of Primary Production 95-99

Compositional Reservoir Simulator Comparison 99-100

CHAPTER 5 STORAGE PERFORMANCE PREDICTION AND ANALYSIS 101

Simulation of Storage Performance with Vertical Wells 101

Simulation of Storage Performance with Horizontal Wells $102-108$

Reservoir and Fluid Properties Evaluation 109-112

Affects of changing the Perm in Top Two Layers of the Original History Match 113-114

Affect of Primary Production Well Location on Original History Match 115-116

Evaluation of Wellbore Effects and Condensate Influence on Condensate Yields 117

Evaluation of Mixing Effects and in the reservoir 118

Storage Operational Design Evaluation 119-120

Evaluation of High Rate and Peaking Tests and Condensate Recovery 120-121

Evaluation of Surface Facility Efficiency 122-123

CHAPTER 6 SUMMARY RESULTS AND DISCUSSION 124-126

$\begin{array}{ll}\text { REFERENCES } & 127-129\end{array}$

APPENDICES 130

Simulator Spatial Properties Views 131-152

EOS Regression Graphs 


\section{LIST OF TABLES}

\section{CHAPTER 3 METHODOLOGY AND OBJECTIVES}

Table 3.1 Comparison of Model Output versus Actual Field Data

\section{CHAPTER 4 RESERVOIR MODEL DEVELOPMENT}

Table 4.1 Sampling Conditions (1979 PVT data)

Table 4.2 Constant Volume Depletion PVT Results

Table 4.3 Constant Composition Expansion

Table 4.4 PR-EOS Case 33 composition breakdown

Table 4.5 Constant Volume Depletion Component Analysis

Table 4.6 Constant Composition Expansion Analysis

Table 4.7 Constant Composition Expansion Analysis after Regression $\quad 75$

Table 4.8 Constant Volume Depletion Analysis after Regression 76

Table 4.9 Material Balance Data 81

Table 4.10 P/z against cumulative wet gas production (Material Balance Plot) 82

\section{CHAPTER 5 STORAGE PERFORMANCE AND PREDICTION}

Table 5.1 Vertical vs. Horizontal Wells Liquid Recovery Comparison 104

Table 5.2 Comparison of Permeability Reductions in Grid Layers 111

Table 5.3 Comparison of Results w/ Original Production Well Location change 116

Table 5.4 Details of High Rate Peaking Schedules Scenarios 121

Table 5.5 Separator Efficiency Evaluation Results 122 


\section{LIST OF FIGURES}

\section{CHAPTER 1 INTRODUCTION}

Figure 1.1 UGS World Working Gas Distribution by Region 8

Figure 1.2 UGS World Working Gas Distribution by Storage Type 8

Figure 1.3 UGS North American Storage- Reservoir Geology 9

Figure 1.4 US North American Storage Fields by Type 10

\section{CHAPTER 2 LITERATURE REVIEW}

Figure 2.1 Gas condensate phase diagram (Schlumberger Oilfield Review 2005) 21

Figure 2.2 Rich and Lean gas liquid-oil saturation curves

\section{CHAPTER 3 METHODOLOGY AND OBJECTIVES}

Figure 3.1 Model Development Flowchart $\quad 24$

Figure 3.2 Two (2) dimensional grid map showing Net Pay of formation 32

Figure 3.3 Three (3) dimensional grid map showing Grid Top of formation 33

Figure 3.4 Cross Section of structure showing Depth of Pay 33

Figure 3.5 Three (3) dimensional grid map showing New Well Locations 35

\section{CHAPTER 4 RESERVOIR MODEL DEVELOPMENT}

Figure 4.1 Quinlan Field Geographic Location 39

Figure 4.2 Monthly Gas production from Quinlan field 1978-2005 40

Figure 4.3 Cumulative Gas production from Quinlan field 1978-2005 41

Figure 4.4 Monthly Condensate production from Quinlan field 1978-2005 41

Figure 4.5 Cumulative Condensate production from Quinlan field 1978-2005 42

Figure 4.6 Onondaga Reef Cross Section 43

Figure 4.7 2D Seismic Lines $(1979,1981$, and \& 1983)

Figure 4.8 2D Seismic Interpretation (1979, 1981, and \& 1983) 45

Figure 4.9 3D seismic proposed areal grid (1998 proposed) 46

Figure 4.10 Three (3D) 730 ms Time Slice Structure Onondaga Formation 46

Figure 4.11 Three (3D) Time Slice of Onondaga Formation 47

Figure 4.12 Summary Core Permeabiltiy, Porosity, Gamma Ray and Lithologic

Description for the Onondoga Formation (Westport Geological Services) 48

Figure 4.13 Whole Core Analysis Onondaga Formation 49

Figure 4.14 Structure Map Top of Onondoga Formation 50

Figure 4.15 Net Isopach Map/Porosity Zones of Onondaga Formation 50

Figure 4.16 NY Geographic Map of Gas Storage $\quad 51$

Figure 4.17 Platform Express Density/ Neutron/ Gamma Ray 54

Figure 4.18 HALS Laterolog/Micro Gamma Ray $\quad 55$

Figure 4.19 Elemental Capture Spectroscopy Tool (ECS) 56

Figure 4.20 Array Digital Sonic $\quad 57$

Figure 4.21 Combinable Magnetic Resonance Tool (CMR) 58

Figure 4.22 PEX/ECS/HADL/CMR ELAN Log 59

Figure 4.23a \% Gas In Place Produced $\quad 69$

Figure 4.23b Produced Gas Gravity $\quad 70$

Figure 4.23c M.W. of C7+ $\quad 70$

Figure 4.23d 2- Phase z factor $\quad 71$ 
Figure 4.23e \% Retrograde Liquid in Reservoir $\quad 71$

Figure 4.24 Comparison of EOS-Predicted and Field Liquid Yields $\quad 72$

Figure 4.25 P WinProp EOS Fluid Properties Analysis Graphs $\quad 77$

Figure 4:26 WinProp CVD Calc Regression Summary 78

Figure 4.27 Original PR-EOS Vol \% Liquid in Reservoir 79

Figure 4.28 Original PR-EOS \% Gas in Reservoir 79

Figure 4.29 EOS Simulation of P/Z versus Cumulative Wet Gas production 81

Figure 4.30 Predicted Storage Liquid Yields 83

Figure 4.31 2D IJ Areal Grid Dimension View 85

Figure 4.32 3D IJK Areal Grid Dimension View 85

Figure 4.33 2D J Layer:17 Cross Section View 86

Figure 4.34 2D I Layer:13 Cross Section View 86

Figure 4.35 3-Dimensional Grid Map of the Reservoir (Subsea Elevation, ft) 87

Figure 4.36 Net Isopach Map/Porosity Zones of Onondaga Formation 88

Figure 4.37 Core Derived Net Pay-Permeability Cross plot 89

Figure 4.38 Core Derived Porosity-Permeability Cross plot 89

Figure 4.39 Porosity Zones of Onondoga Formation 90

Figure 4.40 Liquid-Gas Relative Permeability Curve 90

Figure 4.41 Three Phase Diagram for Fluid Description $\quad 91$

Figure 4.42 History Match Actual vs. Field Data pressures 94

Figure 4.43 Pressure vs. Inv- Actual Field data compared to Model Output 94

Figure 4.44 History Match Actual vs. Field Gas Rate 96

Figure 4.45 History Match Actual vs. Field Oil Rate 96

Figure 4.46 History Match Actual vs. Field Combined Oil \& Gas 97

Figure 4.47 Cumulative CGR (STB/MMSCF) vs. Pressure 98

Figure 4.48 Cumulative STB (bbls/MMSCF) vs. Time 99

Figure 4.49 Condensate Yield during Primary Depletion 100

\section{CHAPTER 5 STORAGE PERFORMANCE AND PREDICTION}

Figure 5.1 3-DGrid Map of the Reservoir with Vertical I/W Wells 101

Figure 5.2 3-D Grid Map of the Reservoir with Horizontal I/W Wells 102

Figure 5.3 Predicted Liquid Production Rates during Storage Operations
with Horizontal I/W Wells

Figure 5.4 Results of Storage Operations Simulation Study with
Horizontal/Lateral I/W Wells

Figure 5.5 Comparison of Liquid Yields during Storage Operation 105

Figure 5.6 Reservoir Pressure as a Function of Gas-in-Place 107

Figure 5.7 Comparison of the Liquid Yields during First Storage Cycle 109

Figure 5.8 Impact of Heterogeneity on the Predicted Liquid Yields during First
Storage Cycle

Figure 5.9 Comparison of Model Predictions and Revised Liquid Yields during First Storage Cycle 113

Figure 5.10 Original Production History Match Comparison w/ Perm Reduction $\begin{array}{ll}\text { in top two layers } & 114\end{array}$

Figure 5.11 Map of Original Production New Well Location change $(14,16,1) \quad 115$

Figure 5.12 Comparison of results with Original Production Well Location change 116

Figure 5.13 Comparison of $\mathrm{CH} 4$ concentrations changes due to Storage Operations

Figure 5.14 Peaking vs. Constant Withdrawal Rate Yield Comparison 121 


\section{CHAPTER 1 INTRODUCTION}

\section{Introductory Comments}

The increased interest in the development of new Gas Storage Fields over the last several decades has created some interesting challenges for the industry. Most existing gas storage fields have been developed from depleted oil and natural gas reservoirs. It is not uncommon for gas storage fields to be developed in water bearing aquifer zones where the presences of conventional type reservoirs are limited. Natural gas condensate reservoirs are typically not found in the eastern United States where most gas storage facilities have been developed close to the colder climates and increased population centers. Therefore, research data is limited on how partially depleted natural gas condensate reservoirs can be developed into dependable natural gas storage reservoirs. Some of the factors that must be considered when targeting depleted oil and natural gas reservoirs for storage development are: effects of mixing of storage solvent gas with residual reservoir fluids, performance of reservoir under storage operations, amount of investment necessary to meet storage contract obligations and surface facility design considerations. A condensate gas reservoir behaves much differently than a depleted dry natural gas or oil reservoir because the residual wet gas liquid is left in the reservoir only to be revaporized and produced by the storage operations much like a secondary recovery operation. Until now documentation of the results of the conversion of natural gas condensate systems to functional natural gas storage reservoirs has been limited due to :

- Lack of available data for analysis due to the small number of natural gas condensate reservoirs converted to natural gas storage reservoirs

- Where available date exists for such reservoirs the use of numerical simulation has not been fully documented and understood. 


\section{Research Goal}

The overall goal of this research study is to look at challenges in the converting a natural gas condensate reservoir into gas storage reservoir and how these affect the overall design of the storage facilities. When a gas storage field is developed typically there is a need to drill more active injection and withdrawal wells to meet the deliverability design parameter. When a field is discovered there is no immediate need to drill excess wells into the field since for production purposes a few wells will suffice. However, when a gas storage field is being developed depending the areal extent and volume of the reservoir a significant number of new wells may be required to meet the deliverability requirements. The wells can be completed in three ways: vertically, directional and horizontally. Each well completion configuration has its benefits and consequences that need to be considered. With a depleted gas condensate reservoir more emphasis needs to be placed on how the well completion will affect field deliverability performance and residual condensate recovery. This research proposes to use a compositional reservoir simulator to predict storage field behavior of a depleted condensate reservoir.

An equation of state (EOS) program was used to initialize the reservoir model with a representative fluid composition that existed before the field was discovered. The initial fluid PVT laboratory results were used in identifying the fluid properties at discovery conditions. After the

fluid was characterized using the equation of state (EOS) model a primary production history match was obtained. The model was then used to perform storage performance predictions using several different design and operational scenarios.

A secondary objective of this study was to utilize the calibrated compositional reservoir model applied to the natural gas storage conversion process to identify operational inefficiencies that can affect storage performance. The ability to use the model to identify these issues will allow for further facility optimization resulting in increased performance. And gain a better understanding how future gas condensate reservoirs will perform as natural gas storage reservoirs. 


\section{Problem Statement}

As it is documented in the literature (Katz et al, 1981) problems arise when a condensate reservoir is converted to storage. These problems are associated with condensate revaporization which complicates the storage field operations and requires special surface facility design. In addition, accurate prediction of condensate yields cannot be achieved by traditional methods. Therefore, it is necessary to better understand the extent and the impact of condensate revaporization on storage performance before the field can be put into service.

The ability to build accurate reservoir models in depleted condensate reservoirs is further complicated because of the pre requisite to develop a fluid characterization model that can be used to simulate storage operations. The reliability of these fluid characterizations coupled with reservoir heterogeneities can make it difficult to provide the answers needed to build a viable storage project.

To address the above described problems this research study will evaluate the issues that can cause unreliable predictions from compositional reservoir simulators and their relative impact on these predictions. The issues with wellbore configurations, operational constraints and factors affecting fluid mixing are just some of the parameters studied in this work.

Another important issue that has to be addressed with gas condensate is the problem of gas quality issues. Natural gas supplied gas storage fields has typically been stripped of the heavier hydrocarbons before delivery to meet requirements. Gas quality continues to be a primary concern in the natural gas storage business because of standards that must be met for gas heating value and maximum impurity limits. Further studies must be performed on gas condensate reservoirs concerning the resultant mixing of the lean storage gas and the rich wet gas. This leads to varying gas compositions that affect the remaining within gas quality tariff standards. These varying gas compositions must be well understood and predicted so that reliable gas stream compositions can be delivered to the customers. 


\section{Objectives}

After the reservoir simulator has been developed and the model has validated the original production history, the storage scenarios can be ran to evaluate the items listed above. Since this project will be in service and operating, the actual storage operating data can be integrated into the reservoir design model to further enhance the predictive capabilities of the reservoir model. It is estimated that full field development will take several years allowing the model to be calibrated and improved over time. The research objectives proposed in this study aim to identify the specific elements that can be related to the development of future gas storage projects from depleted gas condensate reservoirs:

- Develop an understanding of the impact of well configuration particularly horizontal versus vertical designs on the fluid mixing in the reservoir.

- Determine the impact of the reservoir properties on fluid mixing in the reservoir.

- Study how changing operational practices affects the compositional fluid mixing and recovery.

The gas and condensate fluid properties and composition can then be evaluated throughout the primary production period. The effects of storage operations on the reservoir fluids can be simulated to evaluate the degree of mixing and estimated condensate recovery. The reservoir simulator will also be used to optimize the number of wells needed to achieve the desired storage field design requirements. The simulator will also be used to estimate the number of years that condensate production will be a limiting factor in reaching $100 \%$ storage field development.

Other research objectives proposed in this study aim to identify the specific elements that can be related to the development of future gas storage projects from depleted gas condensate reservoirs they are the following:

1. how condensate recovery can be optimized without the need of full gas-liquid compositional analysis.

2. how changing gas compositions during storage operations affect capacity and deliverability projections. 
3. answer if the design of a storage field is impacted by intermediate production history or gas composition of a condensate reservoir.

4. to accurately predict the number of storage cycles and condensate production required with converting to full storage operations.

5. How can condensate production be successfully controlled during storage operations.

6. how does future plant design and key operational parameters affect the hydrocarbon mixing and improve the quality of withdrawn storage gas.

7. How can condensate reservoirs be successfully developed into gas storage reservoirs.

\section{Background}

An Onondaga Reef Limestone (Middle Devonian geologic age) production field was discovered in 1977 and located in the Appalachian Basin. The reef in question is a typical bioherm covering an area of approximately 221 acres with an estimated thickness of 190 feet at a depth of 4500 feet. These bioherms are extinct submarine knolls that were later covered by the Marcellus shales. These reefs present good porosity and well developed vertical and lateral permeabilities.

The initial production well was drilled based on seismic data indicating a reef build up at a depth of 4332 feet. Due to unexpected flows of natural gas only the top few feet of the reef was penetrated with the initial well. The initial well was the only well drilled into the pool and drained the entire acreage over the life of the field. The well had an original production rate of 2.5-3.0 MMSCFD and approximately 100 BPD of condensate. The original shut in reservoir pressure was estimated at $2710 \mathrm{psi}$. The well blew out while drilling due to the high gas volumes encountered in the top of the reef structure. The field has been classified as a cold retrograde condensate reservoir with a bottom hole temperature estimated at $120 \mathrm{~F}$. Most condensate reservoirs have temperatures in excess of $200 \mathrm{~F}$. This phenomenon causes the liquid drop out in the reservoir to be higher in colder reservoir temperatures thus reducing the amount recoverable liquids. An earlier PVT Constant Volume depletion study estimated a recovery of $32 \%$ based on recombined separator liquid products. There is relatively no water production associated with this production field thus the reservoir exhibits no water drive effects. The reservoir fluid properties are much leaner than rich gas condensate fluids increasing the recovery and lowering the percentage amount of condensate dropping out in the reservoir. However, the low oil saturation decreases any mobility and prevents any flow of the fluid in the pore spaces. 
As of August 2005 the total wet gas production was 7.078 BCF and the total condensate recovery was estimated to be 115,507 BBLS. An Equation of State model was used to simulate the primary production where an estimate of the two-phase $z$ factor could be obtained to construct a $\mathrm{p} / \mathrm{z}$ versus cumulative gas production to determine the actual original gas in place and remaining wet gas reserves. The EOS simulation of the P/Z versus cumulative production and regression parameters estimate that the original wet gas in place was 8.056 BCF. The results of the original PVT fluid study, the recent Equation of State(EOS) study and recent compositional reservoir simulation study all indicate that approximately one third of the original condensate liquid in place has been produced. It estimated that there was between 180,000 and 230,000 remaining stock tank barrels in the reservoir.

There was insufficient data to make an estimate of the volumetric initial gas in place because the only well drilled only penetrated the top 20 feet making it difficult to estimate the reservoir pay thickness. There were some estimates of the gross pay thickness based on the earlier seismic data.

The lack of data has made the storage conversion project challenging because many of the design parameters depend on accurate reservoir and fluid characterization. Accurate reservoir was not possible due to only one well was required to drain the entire prospect. This initial well was not cored and was unable to be logged due to problems with drilling the well and the significant flows that resulted.

The geologic reef structure also made it difficult to differentiate reservoir properties since hydrocarbon bearing reefs are not very common in the area and also can have very different reservoir properties and fluid compositions.

After the initial well was drilled and production was started within a couple of months of production an extended compositional PVT studies was conducted. The following tests were conducted: 1) Constant Compositional Expansion and 2) Constant Volume Depletion. One of the main conclusions that came out of the study was that the reservoir was discovered at the dew point pressure of 2317 psi. The maximum liquid phase volume reservoir conditions were estimated to be 2.68\% @ 900 psi and 120 F. There was focused interest in the area after the QE-2 Middle Devonian Onondaga reef well was discovered in 1978. There was speculation on where QE-2 was in located in relation to the reef structure. In 1979 a two dimensional seismic survey (ESI 12-79) was shot from a southwest to northeast orientation that showed that QE-2 
was located on the northeast flank of the reef and to the south and southwest there were more extensive buildups of reef structure. Two (2) more two dimensional seismic lines (ESI 16-81) and (ESI 15-83) were shot in 1981 and 1983.

In 1991 it additional three dimensional (3D) seismic was shot over the entire prospect to further delineate the reservoir boundary limits. This proposal was based on decision to develop the reservoir for storage. Some of the objectives justifying the 3D seismic project were:

1. determine the reservoir boundaries

2. accurate reservoir property characterization

3. determine the best possible place to drill a test well that could be cored

4. aid the directional drill drilling of development wells

In 1999 a test well was drilled and cored. The core analysis indicated a porosity ranging from $3.2 \%$ to $18.4 \%$ with an average of $9.1 \%$. The porosity has been enhanced by dissolution of the reef material forming vuggy pores. The horizontal permeability ranged from 0 to greater than 2 darcies and vertical permeability ranged from less than a millidarcy to 8 darcies.

\section{Gas Storage}

The International Gas Union(IGU) Triennium 2006 - 2009 Underground Gas Storage(UGS) report by Wallbrecht reports that there are a total of 630 UGS facilities in the World of which 389 are in the United States. Of the 389 total 307 or $79 \%$ are from Depleted oil and gas fields with the majority being depleted natural gas reservoirs. The remaining are from Aquifers and Salt Caverns. The United states has $3.9 \operatorname{TCF}\left(110.7 \times 10^{\wedge} 6 \mathrm{m3}\right)$ or $34 \%$ of working gas out of world total of $11.4 \mathrm{TCF}\left(322.5 \times 10^{\wedge} 6 \mathrm{~m} 3\right)$. The total world deliverability withdrawal rate is 183.7 BCF/day $\left(216.810^{\wedge} 3 \mathrm{~m}^{3} / \mathrm{h}\right.$ ) from approximately 22,728 storage wells. See Figure $\mathbf{1 . 1}$ below for the UGS Working Gas Volume Distribution by Region. 


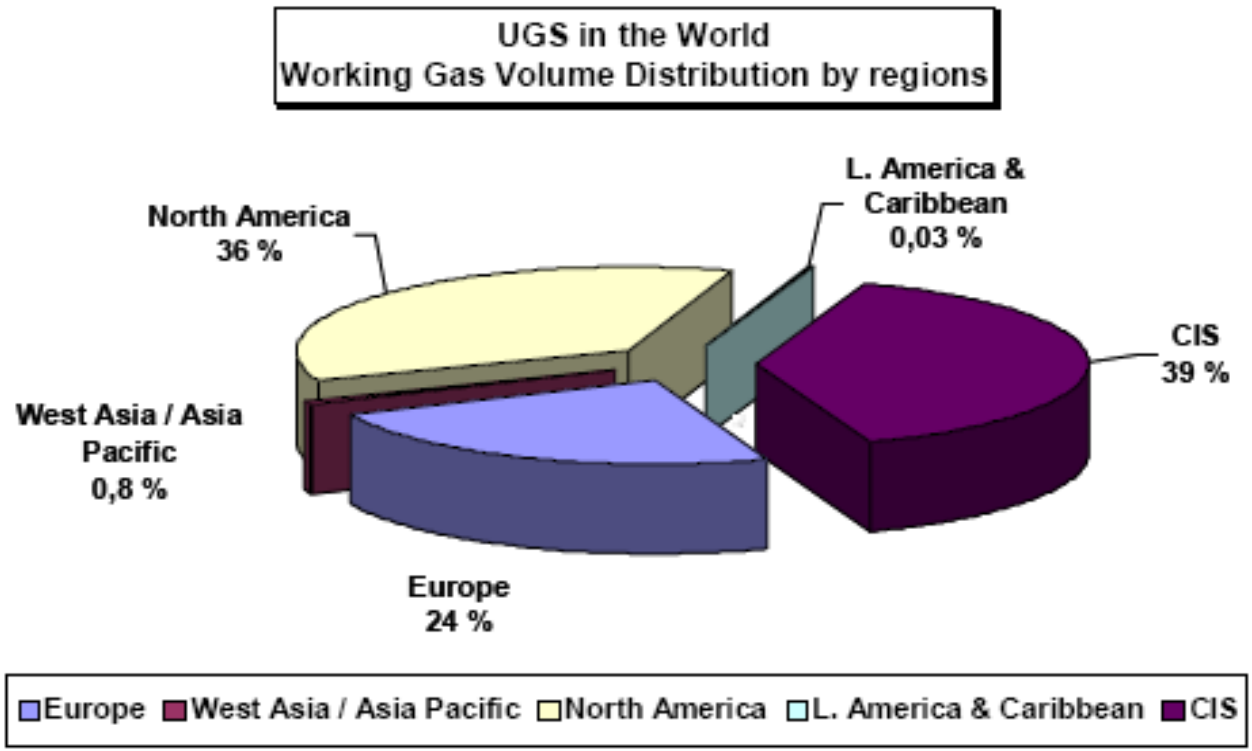

Figure 1.1 UGS World Working Gas Distribution by Region

It has been estimated that the global working gas volume distribution of Underground Gas Storage (UGS) reservoirs types in the world with the majority (78\%) being in depleted gas reservoirs and $5 \%$ in depleted oil reservoirs. The remaining is in aquifer $(12 \%)$ or either salt or mined caverns (5\%). See Figure 1.2 for a chart showing UGS World Working Gas Distribution by Storage Type:

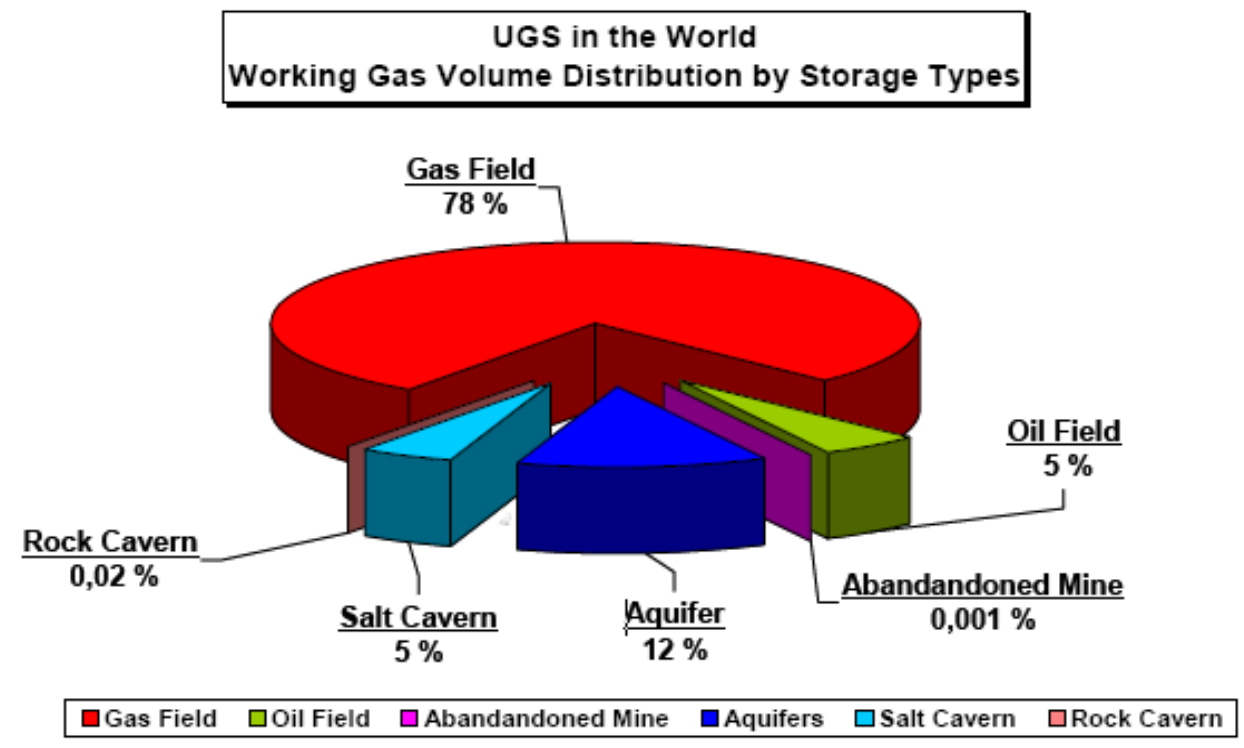

Figure 1.2 UGS World Working Gas Distribution by Storage Type 
Carbonate reservoirs typically have better reservoir qualities and are preferred for storage development over traditional sandstone sedimentary structures. Carbonate reservoir geologic structures comprise approximately 40 \% (1.3 TCF/to 3.1 TCF) of all storage reservoirs in North America. Figure $\mathbf{1 . 3}$ below shows the North American Storage- Reservoir Geology distribution.

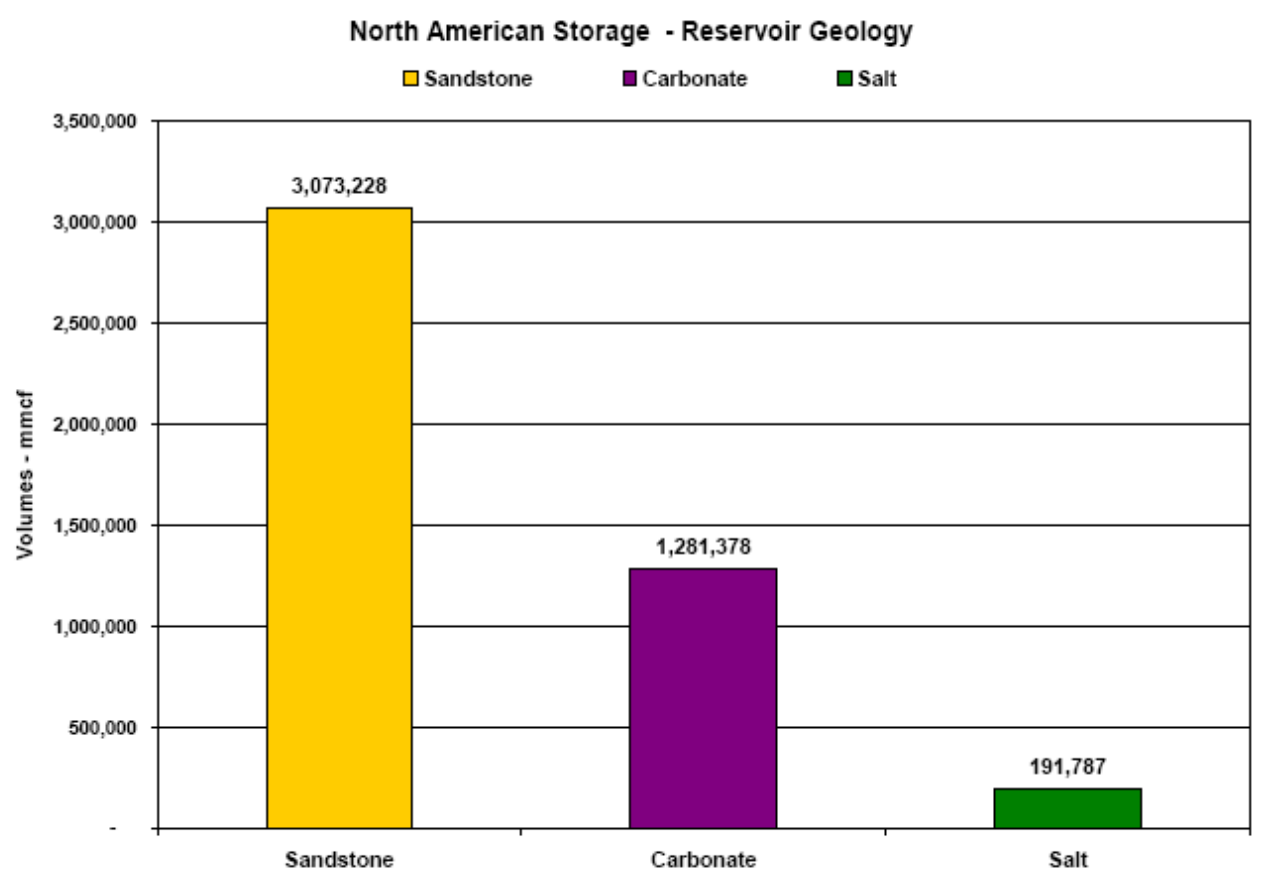

Figure 1.3 UGS North American Storage- Reservoir Geology

The Survey of Underground Storage of Natural Gas in the U.S. \& Canada, (2007) indicates that there are a total of seventy-six (76) designated carbonate storage fields in the United States. They comprise approximately $20 \%$ of all storage reservoirs in the United States. Therefore their occurrence is substantially less than sandstone reservoirs.

The current ratio of working gas to total gas(cushion and working) in North America is 2.89 TCF/5.62 TCF or $51.5 \%$ for Depleted Oil and gas and 11.4 TCF/24.8 TCF or $45.8 \%$ for all storage facilities in the world. The carbonate reef reservoirs will allow for the above average ratio of Working gas to Cushion gas since these type reservoirs are usually very prolific. The same statistics for carbonate fields in the United States shows the current ratio of working gas to total gas(cushion and working) in the United States is 0.91 TCF/1.43 TCF or $63.6 \%$. 
Of 307 total United States Gas storage fields developed from Depleted Oil and Gas Fields only seventy-six(76) are in Carbonate type reservoir structures. Only nine (9) of the carbonate storage fields have been developed in depleted natural gas condensate reservoirs. Figure 1.4 shows the current US North American Storage- reservoir geology type.

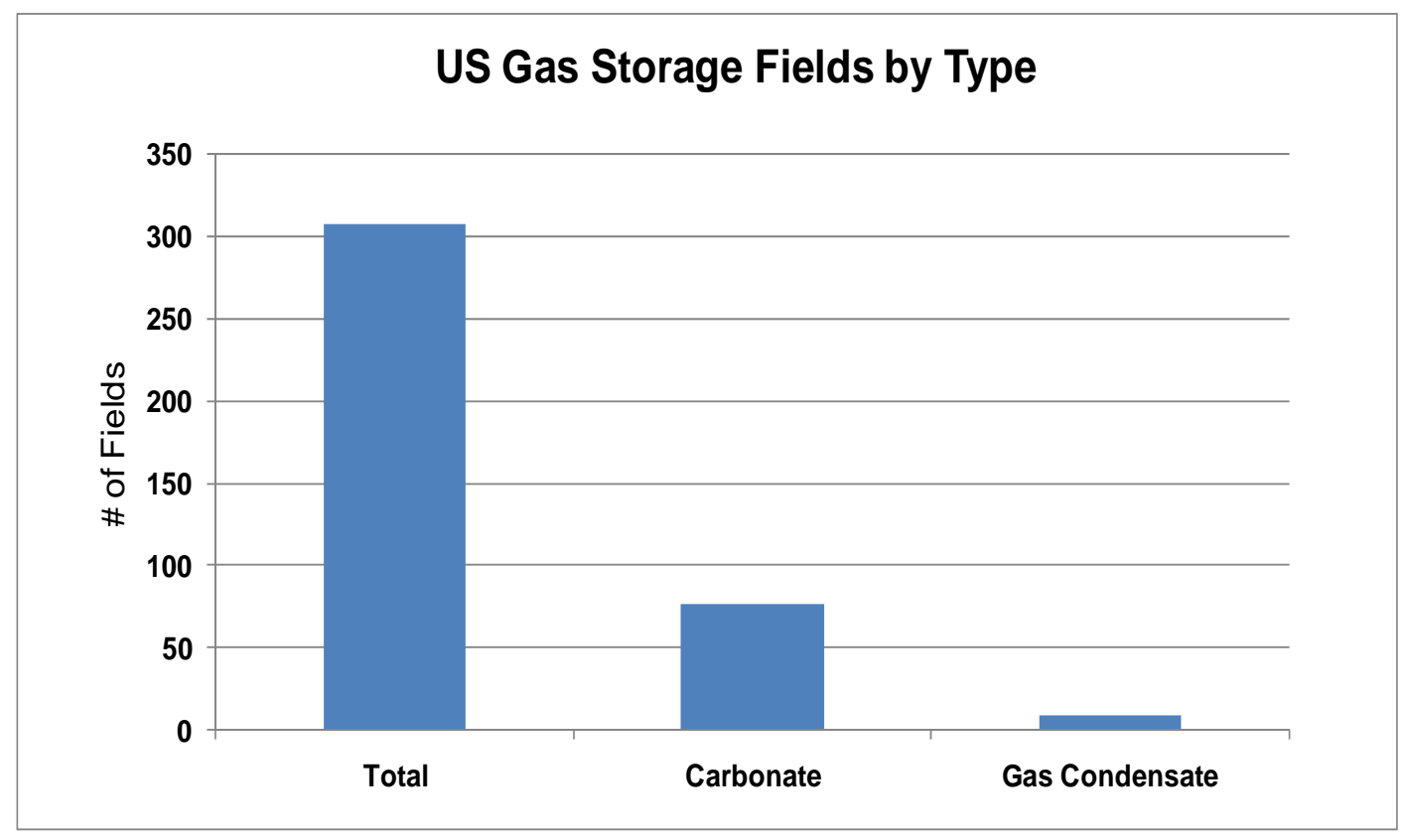

Figure 1.4 US North American Storage Fields by Type

Most are developed in the Salina-Niagaran pinnacle carbonate reef formations found in the Michigan Basin on North America and south central Ontario region of Canada. All of these make up the total carbonate storage reservoirs developed in depleted natural gas condensate reservoirs in North America. Some of these formations are the A-2 Carbonate, Dundee, Niagaran/Guelph and Salina Niagaran reef formations.

Carbonate gas condensate fields in the United States show the current ratio of working gas to total gas(cushion and working) in the United States is 0.16 TCF/0.20 TCF or $80.0 \%$. The requirement for less base or cushion gas makes carbonate gas condensate extremely attractive for storage development. 
There are only seventy-eight (78) injection /withdrawal wells in these carbonate gas condensate storage pools. This is one indication of how prolific the carbonate reefs and how well they perform as storage reservoirs. Horizontal and directional drilling strategies utilized in the development of these fields are another reason for the small number of wells required.

One important trend cited in the IGU UGS Database Study (2009) is the increased use of horizontal wells to meet growing deliverability requirements of future and existing storage facilities. This study seeks to research the effects of wellbore configuration on deliverability from the facility.

Internationally according to Wallbrecht in the IGU UGS Triennium 2006 - 2009 Report only two(2) other countries: Germany and CIS/Ukraine have natural gas storage in depleted gas carbonate condensate reservoirs. The reservoir as part of the dissertation study is the Devonian age Onondaga limestone reservoir.

Although carbonate reservoirs make prolific storage reservoirs their relative small numbers make them some of the least understood technologically. The items described above concerning gas quality, horizontal well performance, working gas/base gas ratios and fluid mixing effects are addressed in this study. 


\section{CHAPTER 2 LITERATURE REVIEW}

The research on the conversion of depleted natural gas condensate fields to natural gas storage projects indicated that there have been very few papers dealing with the conversion process. However, there have been several papers and work done in the field of phase behavior associated with gas condensate production fields. Some of this work has been indirectly applied to gas storage through the secondary process of gas re-injection and cycling for improved condensate recovery. There are few papers that directly address the conversion process from gas condensate to gas storage.

The approach taken to review all applicable literature pertatining to the research topic presented here will look at the following:

1. use of compositional simulators to study natural gas condensate reservoirs in production or gas cycling applications

2. reservoir fluid charcterization associated with natural gas condensate reservoirs documented work

\subsection{Gas cycling/modeling literature}

One of the most important papers SPE-10166 by Katz et. al titled "Predicting Yield of Revaporized Codensate in Gas Storage" (1981) is one of the most important papers in existence today dealing with Gas Storage affects on Condensate reservoirs. This paper looked at the problems associated with developing an adequate phase behavior model and the problems with not having a reservoir model to use to study the mixing affects on condensate recovery.

In Katz et. al SPE Paper(1981) the data from two (2) Niagaran bioclastic carbonate reef pools in Michigan were used with phase behavior models for gas condensate systems to predict condensate yield and recovery during storage operations. The initial results indicated that mixing and revaporization of the condensate in the reservoir was affecting the liquid yield recoveries. The reservoir properties of the Salina-Niagaran reefs in this paper are very similar to the Devonian age reef reservoir presented in this dissertation. The use of phase behavior packages have been successfully used for sometime to predict condensate gas ratios and liquid yield recoveries during primary production. There use in predicting mixing of the storage gas with residuel liquid condensate is less understood. Katz et al concluded that acurate 
compositions of the resevoir gas and retrograde liquid are necessary to make reliable gas storage operation revaporization and resulting liquid yield recovery predictions. Furthermore, accurate recombination of the seperator gas and liquid well streams and extended compositional analysis is very important before any phase behavior and mixing effects can be analyzed. The retrograde liquid in the two(2) fields in the study at the time of conversion was 4$5 \%$ much like the amount left in the reservoir in this dissertation.

The primary condensate gas ratios(50-10 bbls/mm )for the two(2) Niagran reefs are very close to the CGR's for the Quinlan reservoir presented in this disseration(40-15 bbls/mm). The reservoir pressure was approximately 3500 psi and the gas content was between 15 and 26 bcf compared to the $8 \mathrm{BCF}$ and 2300 psi pressure in the Quinlan resevoir. The primary condensate recovery was 427,000 and 255,000 STB for the reservoir in this paper compared to the 100,000 bbls condensate production in th reservoir in this dissertation. The reservoir temperature of the two(2) Niagran reefs(114 F and $118 \mathrm{~F}$ ) in Katz et al(1981) papar were similar to the reservoir temperature in the Quinlan reservoir(120 F). This is relatively cold for most condensate reservoirs found in the world.

The paper by Katz et al in this study modeled the affects of using alternate modes of operation and how this would affect the mixing of the gas and liquids in the reservoir. Mode 1 looked at using the same wells for injection and withdrawal to study the condensate recovery and mixing effects. Mode 2 looked at the sweeping affect by injecting in one part of the reservoir and withdrawaing from the opposite end of where the injections took place. A final Mode 3 could be studied to see if withdrawing mixed storage from the main part of the pool for peaking purposes could be achieved with lower condensate yields as a result of combining with Mode 2 operations.

The general finding of the study indicated that the phase behavior model used in the study overestimated the condensate production under actual storage injection and subsequent withdrawal operations. The difference in predicted and actual condensate recoveries in this paper was thought to be due to inadequate mixing in the reservoir under different modes of operations.

The Katz SPE paper did not take into account the affects of the reservoir properties such as permeability and geologic structure characterization on the liquid recovery. 
The second paper by R.A Herzog's was "Retrograde Vaporization of Residual Condensation in Storage Field Development "Operating Section Proceedings, American Gas Association Baltimore, Maryland (1980) T187. R.A. Herzog worked for ANR Storage Company and was instrumental in the development of their Niagaran Reef Storage fields located in Michigan he concluded that since retrograde liquid can form as a result of pressure depletion then the fluid should be re-vaporized on re-pressurization by gas storage operations. He further explained that the depletion phase envelope will determine how much condensate will be present at the time of conversion to storage. He also stated that the phase envelope will not be constant and will change with decreasing pressure as a result of production and increasing pressure as a result of re-pressurizing for storage. The number storage cycles will affect the reservoir fluid composition and mixing scenarios. At some point in each pressure depletion cycle the condensate liquid will drop out in the reservoir depending on the phase envelope. It was also stated that the storage gas will continually be enriched by the revaporization and production process making it necessary to monitor the heating value of the gas. The reservoir properties were thought to have an effect on the condensation and revaporization processes acting as a separator. The mixing effects were first presented as a factor in determining the produced gas compositions and the resulting need for adequate surface facilities to handle the fluids. This dissertation seeks to explain this phenomenon due to the increased gas compressibility as a result of the removal of the heavier hydrocarbons and the conversion to $100 \%$ storage gas.

In SPE Paper 9995 "Overview of Phase Behavior in Oil and Gas Production “ Donald L. Katz, SPE University of Michigan SPE 9995(1982) identifies the use of the Peng Robinson Equation of State model developed in 1976 to be an improved way of determining multi component system phase behavior. Again Katz discusses the Gas Storage cycling affects on the partially depleted condensate reservoirs caused by gas storage operations but does not study the effects of the reservoir properties. This can only be done using compositional reservoir model proposed in this dissertation. Another important factor for consideration is the viscosity of the condensate liquid in the reservoir. It was shown that interfacial tension caused be viscosity variation in the fluid can cause relative permeabilities of the various phases to be affected.

Katz also again talks about the significance in the capturing the revaporized condensate in the well streams associated with gas storage operations. His experience with quantifying the condensate production was troubling because of the inefficiencies in the two phase gathering system related to liquid and gas measurement issues. 
In "Simulation of Gas Condensate Reservoir Performance" SPE Paper 10512 by Keith H. Coats published in $1983 \mathrm{JPT}$ the first indications of attempting to explain gas condensate phase behavior coupled with reservoir simulation. Gas cycling was used to study the EOS and mixing affects of revaporization on the condensate fluid in the reservoir. An important outcome of Coats work was that full compositional modeling is necessary for accuracy where cycling pressures are below the initial dewpoint pressure of the system. He concluded that the black oil models and full compositional model show good agreement for very rich condensate fluids. Also, it was shown that black oil simulation can be used where the fluid composition is reduced into a two component system where multi component fluid phase behavior can be ignored. However, the work was only in 1D dimension and did not consider the 2D and 3D dimensional affects of spatial reservoir property variations.

The most current paper addressing the use of a full compositional reservoir model was SPE Paper 106341 written in 2006 "Simulation of Underground Natural Gas Storage in Sarajeh Gas Field, Iran" by E. Khamechi and F. Rashid, Tehran Polytechnic. This was a study to look at the conversion of the Sarajeh natural gas condensate reservoir located in Iran to Underground Natural Gas Storage(UGS). Also, the Sarajeh reservoir was thought to have a Gas Water contact unlike the reservoir proposed in this study that produced no water during the production history. The limestone reservoir in the Qom formation was discovered in 1959 at a pressure of 5699 psi at $5800 \mathrm{ft}$ and $228 \mathrm{~F}$. Based on the available production data the field had produced 59.5 BCF and 2.78 million bbls of condensate until 2003.

A coarse grid model was proposed to be built and simulations ran to history match the reservoir production history. The studies also proposed to determine how many vertical and horizontal wells would be required to meet the deliverability requirements from the storage facility. A compositional reservoir simulator was used and the Peng Robinson EOS was used for the studies. The conclusions were that they could achieve a good history match, determine the number wells required for a certain deliverability requirement and calculate ultimate recoveries of gas and condensate from the field using the simulator. The pressure support strength of the aquifer could not be integrated because of the need for fluid level data for into the model. 
The Third SPE Comparative Solution Project: Gas Cycling of Retrograde Condensate Reservoirs performed in August 1987 published in the Journal of Petroleum Technology by Douglas E. Kenyon and G Alda Behle presented: The Numerical Simulation Symposium sought a compositional modeling problem. Numerical comparison of PVT data was thought to be very important. Nine companies participate in the study of gas cycling in a rich retrograde gas condensate reservoir. In summary surface oil rate predictions differed initially but agreed better later. There are two major parts to a compositional reservoir model study; the PVT data and the reservoir grid. In this paper a major conclusion was there was considerable disagreement about the condensate saturation near the producing nodes. This was thought to have been caused by convection and subsequent deposition near the lower pressure areas.

There also was a wide range of variance in oil liquid saturation calculations versus pressure under CVD(Constant Volume Depletion) tests. The peak dew point saturation was calculated to be approximately 2500 psi and varied by 18 to $22 \%$ from the initial PVT Laboratory data analysis. The final data had about the same discrepancy however the initial data matched fairly well.

Abbas Firoozabadi SPE Reservoir Engineering Research Institiute and Mashhad Fahes. Imperial College presented in paper from December 2007 SPE Journal Page 407 "The wettabiltiy alteration to Intermediate Gas Wetting in Gas Condnesaste Reservoirs at High Temperatures". They discussed that in many gas condensate reservoirs that liquid accumulation around the wellbore at higher temperature can significantly reduce deliverability. Also, they studied to see if at the liquid saturations below $5 \%$ in the reservoir well below the point of fluid flow and mobiltiy in the reservoir could be affected by condensate adsorption to the porous media. Both near wellbore and at the reservoir extents there is a concen that condensate blockage would affect our deliverability requirements and total capacity estimates.

\subsection{EOS/Fluid Characterization/Sampling literature}

In "Predicting Phase Behavior of Condensate/Crude-Oil Systems Using Methane Interaction Coefficients" SPE 6721 in 1978 by D. L. Katz, SP\&AIME, U. of Michigan and A. Fioozabadi, Abadan Institute of Technology it was concluded that for gas-condensate systems, the PengRobinson-AGA procedure with extended analysis and interaction coefficients for methane and C6+constituents correlated with density seems to give the most reliable results. 
In "Gas Condensate PVT - What's Really Important and Why?" Curtis H. Whitson, Øivind Fevangb and Tao Yanga from Norwegian U. of Science and Technology (NTNU) and PERA presented at the IBC Conference(1999) said the following about gas condensate reservoirs“ from an engineering point of view two additional issues which must be addressed in a gas condensate reservoir " they are:

- How the condensate "yield" will vary during the life of a reservoir, and

- How two-phase gas/oil flow "near" the wellbore affects gas productivity.

He said the above two issues are very important relating to the PVT properties of the fluid system but the productivity is more related to the reservoir properties and relative permeability fluctuations. Some of the important PVT properties of condensate gas systems that must be considered are; gas compressibility factor, gas viscosity. Compositional (C7+) and the effect pressure has on the composition along with the effect of oil viscosity on liquid percipitation must area other properties that need studied before accurate analysis can be made.

- Material balance equations, estimation of hydrocarbon in place, dry gas, wet gas, gas condensate, depletion, active aquifer, high pressure, high temperature.

- Modeling gas condensate reservoir fluid systems with an equation of state is discussed, as is EOS modeling of complex fluid systems with strongly varying compositions and PVT properties.

Dewpoint or saturation pressure of a condensate system is implicitly defined by the pressure dependence of composition of the system. It has been said that the dewpoint of the system is less critical than previosly thought because the PVT properties listed above are more important in reservoirs where pressure depletion is occuring. It is more important to understand the phase behavior affects of revaporization and liquid formation below the dewpoint because the pressures are much less than the initial dewpoint of the systems.Accurate PVT modeling is very important to the success in modeling fluid communciation or adsorption and how this affects oil and gas surface volume predictions.

Whitson explains that: A PVT model may not be capable of accurately describing all PVT properties with equal accuracy over a fairly wide range of conditions. EOS models have been shown to not be very effective in matching the retrograde phenomena specifically gas 
compostional behavior and liquid formation of the system near or just below the initial dewpoint. The retrograde behavior of the fluid is not well established at the fringe of the gas liquid interface affecting where the actual condensation of liquid begins occur. This phenomna can have a either a well defined or have very little effect. Therefore the determination of the initial dewpoint can be shown to not have a significant impact on the phase behavior studies.

In summary, Whitson explains that

- For gas cycling projects above the dewpoint, PVT properties have essentially no effect on condensate recovery because the displacement will always be miscible. Only the definition of initial condensate in place is important. Gas viscosity has only a minor effect on gas cycling.

- For gas cycling below the dewpoint, the key PVT properties are Z-factor variation during depletion, $\mathrm{C} 7+$ content in the reservoir gas during depletion, and $\mathrm{C} 7+$ vaporized from the reservoir condensate into the injection (displacement) gas.

Whitson and Torp in SPE Paper 10067 March 1983 "Evaluating Constant Volume Depletion Data" said that predicting condensate recovery is problematic due to the mixing of the two phases. The flowing gas containing retrograde liquid mixes with existing liquids when the pressure declines and the flowing oil has solution gas dissolved that when the pressure is reduced vaporizes and mixes with the existing gas phase. This phenomenon cannot be simulated in the laboratory or with complex component phase behavior models. The Peng Robinson EOS calculated liquid densities from the simulated CVD (Constant Volume Depletion) data were always lower than the laboratory measured values. This is one of the reasons they concluded that the EOS programs overestimate liquid volumes from reservoir fluids. Another conclusion from the study was that the PR EOS overestimated Solution gas Oil ratios (Rso, SCF/STB) because of the lower or underestimated liquid densities. This is another reason this dissertation embarked on using a reservoir to model physical flow in the reservoir in an attempt account for this and not solely on that liquid and gas compositions are only dependent on pressure. 
Gradient models that designate a composition at a specified reference depth at a given pressure and temperature cannot be used in very low oil saturated gas condensate reservoirs where the mobility of the fluids is minimal. Their ability to predict phase behavior and fluid flow in porous media is limited.

In SPE 67283 "Experimental Investigation Into Revaporization of Retrograde Condensate" 2001 Kai Luo, Shi Li, Xitan Zheng, Gang Chen, Ning Liu, Wenyue Sun, Research Institute of Petroleum Exploration and Development indicated the following findings:

- The gas injection experiment after depletion both in the long-core apparatus and in the PVT cell shows that the revaporization of gas condensate in the reservoir condition is effective, the dry gas injected will vaporize efficiently not only those intermediate hydrocarbons but also some heavy hydrocarbons (C20+). For gas condensate reservoirs with high dew point pressure, it is possible to use gas injection to vaporize the retrograde condensed oil produced in the depletion exploitation.

- The cumulative condensate recovery in long-core apparatus are higher than that in empty PVT cell at the same gas injection volume, which indicates that the porous media may be helpful to the revaporization process.

- The long-core gas injection experiment shows that the oil produced are heavier in the gas injection above the saturation pressure than that in gas injection below the saturation pressure.

- During the gas injection at the reservoir pressure, the dry gas injected will exchange components with the original gas condensate and lead to a rise in dew point pressure and retrograde condensation, which will lower the gas condensate recovery to some extent. 
From Schlumberger "Understanding Gas Condensate Reservoirs" Oilfield Review Winter 200506 a case study was presented concerning condensate blockage in the Vuktyl gas condensate field in Komi Republic, Russia. The field has been in production since 1968 and had an initial reservoir pressure was 5200 psi and $142 \mathrm{~F}$ with 15 TCF total gas reserves and 1.2 Billion bbls of total condensate reserves. Condensate blockage was thought to have reduced the deliverability from the carbonate reservoir. Gazprom decided to try to produce the field with no gas cycling utilization. In 2006 the approximate recoveries were estimated to be $83 \%$ of the gas and $32 \%$ of the condensate at a reservoir pressure of approximately 500 psi. It was decided to start a secondary recovery process where a mixture of propane and butane would be injected into the reservoir as a form of a solvent bank. Production logging indicated that the two phase gas flow was present and the solvent bank had only effectively recovery $0.4 \%$. Another pilot project was started where dry gas was injected but this became ineffective due to the large amounts of gas that had to be injected to recover minimal amounts of liquid condensate. The most promising recovery technique seems to be the combination of injecting a solvent bank followed by dry gas. The thought is when the mixture is produced back the dry gas will have reduced the condensate blockage issues near the wellbore. This dissertation will look at the problem associated with condensate blockage and see if this interferes with gas storage deliverability requirements.

From Schlumberger "Understanding Gas Condensate Reservoirs" Oilfield Review Winter 200506 explains that Gas Condensate reservoirs are called "retrograde" condensate reservoirs because they exhibit the opposite or reverse fluid behavior than expected. When production begins the reservoir pressure decreases isothermally at the dewpoint and crosses the equilibrium line of $100 \%$ gas and starts to drop out a percentage of the liquid phase in the reservoir. The amount of this liquid is primarily dictated by whether the original multi component system is classified as rich or lean. The lean system would drop out a minimal amount of liquid in the reservoir as the pressure is decreased. The amount of the liquid phase in the reservoir is a function of the pressure, temperature and composition of the fluid. 
Figure 2.1 shows the gas condensate phase diagram and Figure 2.2 shows the liquid drop or oil saturation curve for lean and rich gas condensate systems:

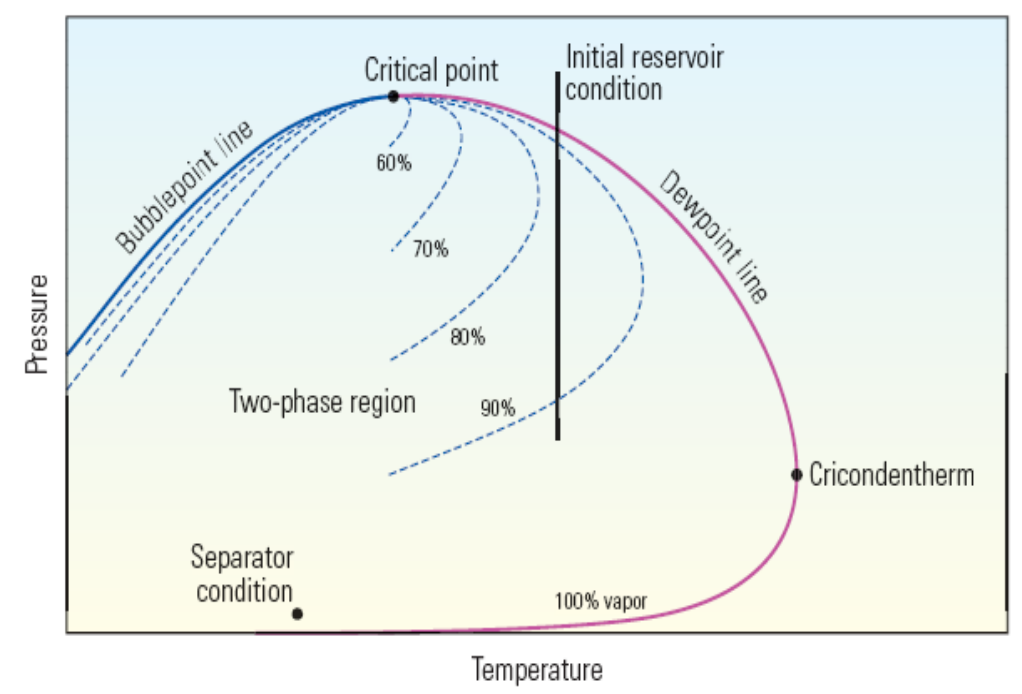

Figure 2.1 Gas condensate phase diagram(Schlumberger Oilfield Review 2005)

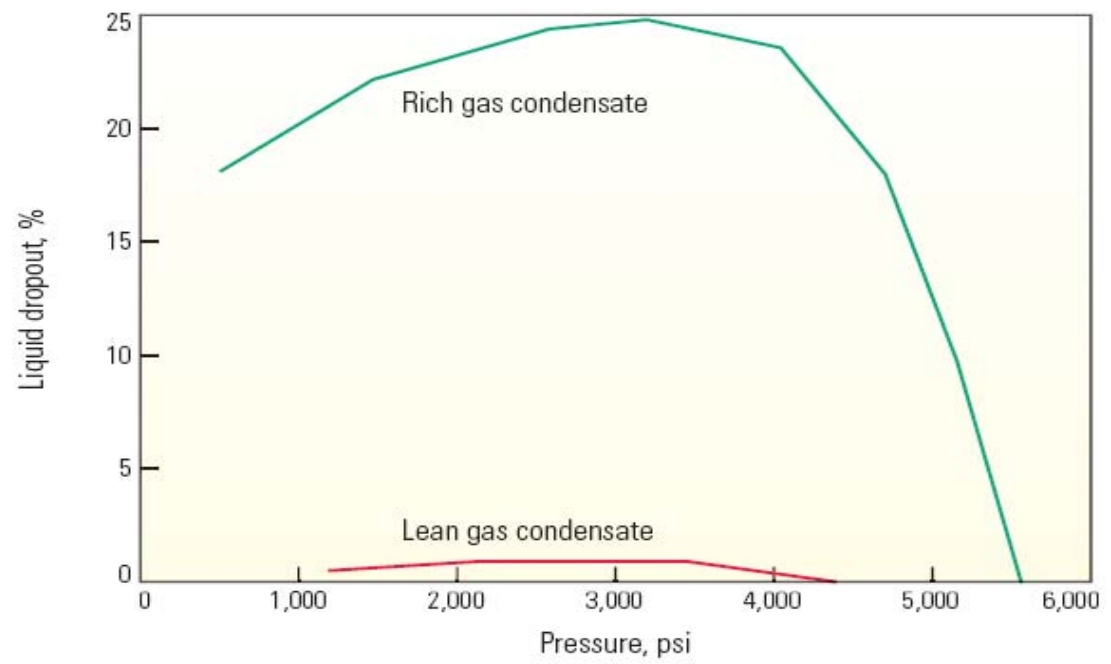

Figure 2.2 Rich and Lean gas liquid-oil saturation curves(Schlumberger Oilfield Review 2005) 
It is very difficult to obtain a sample of the reservoir fluid at reservoir conditions. However it is easy to collect gas and liquid samples from the surface separators and recombine them into a representative well stream composition for analysis in the laboratory. There are problems that can arise from trying to recombine the surface collected samples into a representative reservoir fluid.

1) the sample can become contaminated due to mishandling in obtaining the samples

2) depending on the time of sampling rate fluctuations can provide false samples

3) the pressure at the time of collection can affect the gas and fluid phases

4) condensation in the production strings will affect the ratio

5) evaporation of the liquid in collection

6) samples must be obtained at pressures above the dewpoint

7) large drawdowns drop out condensate around the wellbore artificially increasing the gas oil ratio.

8) Separator issues dealing with changing rates resulting in liquid in the gas outlet and gas in then liquid outlet.

In the case of the work in this dissertation there was a question about whether the laboratory analysis of the initial gas liquid composition was correct. Our fluid property analysis did not agree as did previous intermediate studies leading to the belief the initial reservoir fluid samples may have been compromised. This work will show that it is possible to achieve accurate predictions for the primary production history matching and condensate yields as a result of storage operations.

Newer methods such as split stream and in line separation can be used today to provide better samples for laboratory recombination analysis. Split stream sampling uses a smaller sample flowed through a manifold that eliminates the separator problems. Isokinetic sampling can also be used where a separate mixing chamber is utilized. 
There have been few papers written that directly address the conversion process of a natural gas condensate resevoir to gasstorage. This doctoral study topic was chosen primarily because of the present need to convert an existing natural gas condensate field to storage requiring more current and comprehensive study that does not exist in today's expansive literature. The work in this study will address some of the major concerns with the development of these carbonate gas condensate reservoirs and describe how they affect the overall development plan. A full compositional reservoir model along with an Equation of State model to capable of characterizing the reservoir fluids and mixing interactions as a result of gas storage operations will be used. Many of the topics of the collection of papers described in this literature review shows that only these full compositional reservoir models can be used to simulate the near well bore effects and effect of reservoir parameters on the results.

In summary, there has not been very much literature and studies performed on the effects of revaporization of the residual condensate by re-pressurizing and converting a gas condensate reservoir to natural gas storage. Specifically, there is limited work where the reservoir effects are considered on the retrograde behavior of the reservoir fluids and the resulting mixing process. 


\section{CHAPTER 3 METHODOLOGY}

The primary objective in this work was to develop a compositional reservoir simulator that could be history matched to the primary production of both in gas and liquid volumes of a gas condensate reservoir. The first step in creating a compositional reservoir model is to develop an initial reservoir characterization from available previous initial PVT studies and extended compositional analysis. A secondary objective of the study was to demonstrate that the model could be used to evaluate certain facility operational changes. The following flowchart Figure 3.1 was used in study as a guide to develop a fully functional compositional reservoir model to be used for storage scenario predictions.

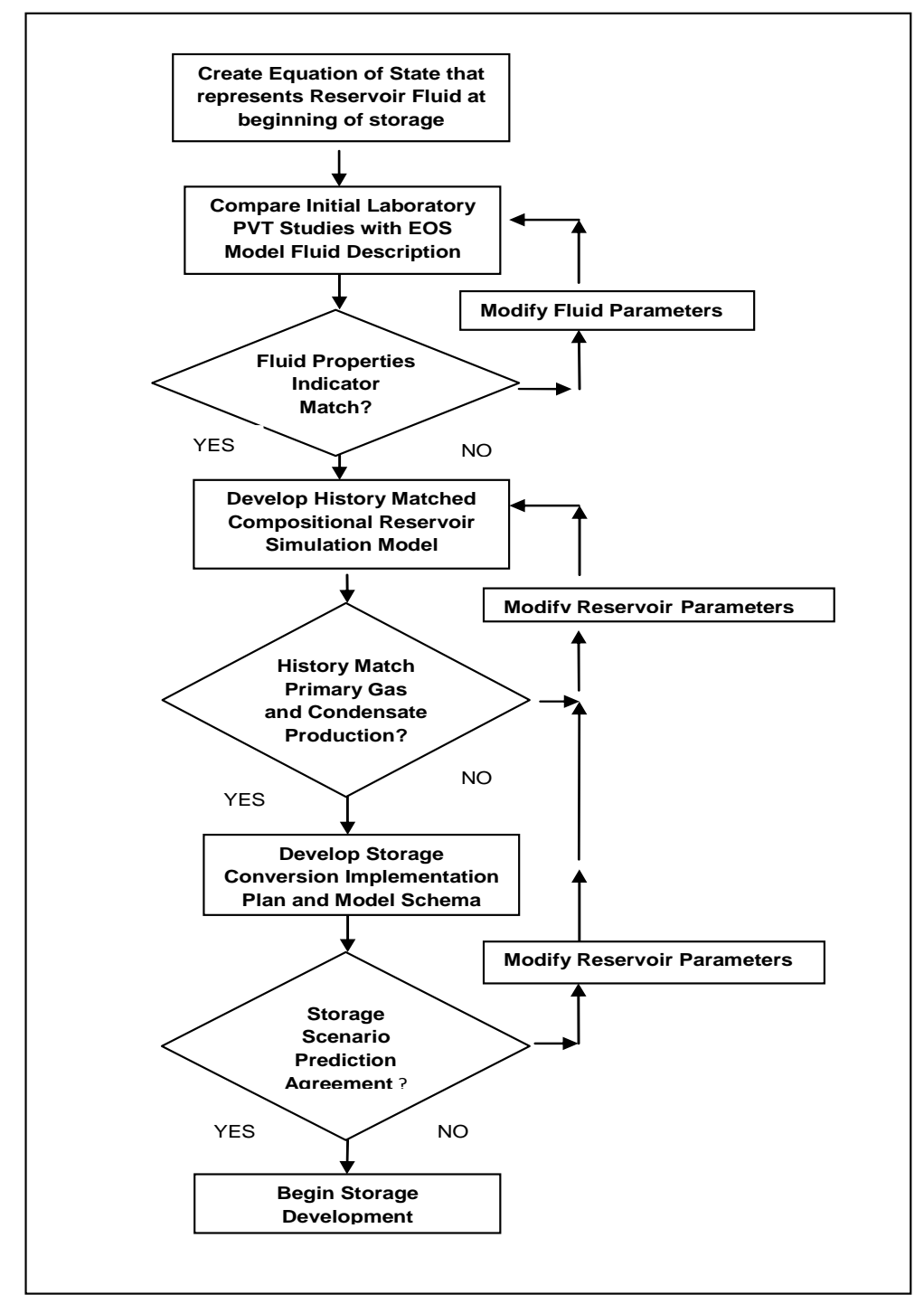

Figure 3.1 Model Development Flowchart 
The Methodology for this study has been broken down into the following steps:

1. Use an Equation of State model to simulate retrograde condensation and revaporization in the reservoir and to predict the liquid yield during storage withdrawal cycles

2. Develop a compositional numerical reservoir simulator and an Equation of State (EOS Model) for the multi-component system. Use these tools to evaluate the degree of mixing. The degree of mixing is dependent on a number of factors including well configuration, residence time, reservoir structure, injection and withdrawal rate schedules/patterns.

3. Evaluate several storage designs and scenarios and study how these impact condensate yields during storage operations. This will enable operations to efficiently manage the storage field.

Katz et. al SPE Paper(1981) concluded, as described in the literature review chapter that understanding the critical parameters that affect the mixing of the fluids in the reservoir is necessary to describe how they have a direct affect on the predicted and actual condensate yield recoveries. These parameters have been studied in this research and shown to influence the predicted condensate yields.

One of the key parameters for storage facility design and operation is the liquid yield during the storage withdrawal cycles. A Peng-Robinson PR-EOS phase behavior package was utilized to simulate retrograde condensation in the reservoir and to predict the liquid yield during storage withdrawal cycles. Literature research indicated in Katz et al. (1978) that the PR-EOS is the best solution for natural gas condensate systems. The following steps were followed in this part of the study:

1. Comparison with Laboratory PVT Study

2. Comparison with Primary Production History

3. Material Balance Study

4. Simulation of Storage 


\section{Comparison with Laboratory PVT Study}

The volumetric performance of gas condensate reservoirs is an essential requirement for optimum design and operation of equipment involved in production, transportation, and processing facilities. The performance of gas-condensate reservoir is strongly compositiondependent therefore, it is necessary to predict reservoir fluid compositional changes under varying pressures and depletion processes. Normally, a PVT study is performed on the reservoir fluid to define the fluid properties at reservoir conditions and at surface separation conditions. For gas condensate, laboratory studies generally conducted are Constant Composition Expansion (CCE) and Constant Volume Depletion (CVD) tests. CVD tests are considered to be representative of the primary depletion and retrograde condensation behavior of gas condensate reservoirs. Data generally obtained from this test are dew point pressure, gas produced, liquid dropout and gas deviation factor at various pre-determined pressure steps ranging from discovery pressure to separator pressure. Detailed PVT studies are costly, tedious and time consuming. Consequently, an Equation of State (EOS) is used to match the reported experimental data. Once matched, the EOS can be used to predict the fluid properties over a wide range of pressure and temperature conditions. The EOS approach is most commonly used for natural gas systems due to its applicability at high pressures for both liquid and vapor phases. Probably the most successful EOS for natural gas property calculation is the one proposed by Peng and Robinson (PR).

The primary input data to the EOS is the composition of the reservoir fluid in terms of mole percent. It naturally follows that if the input data set is incomplete or inaccurate, the subsequent matching with EOS will be problematical. The PVT study includes the compositional analysis of the reservoir fluid in terms of mole percent. The compositional analysis usually ends up with the undefined plus fractions, commonly known as the C7+ fractions, which contain an indefinite number of components with a carbon number higher than six. The molecular weight and specific gravity of the plus fraction may be the only measured data available. A number of papers report comparisons of PR-EOS and laboratory PVT results for gas condensate. Most of these reports emphasize the C7+ characterization as the key element in attaining agreement between EOS and laboratory results. Numerous authors have published papers about how to characterize the plus fraction. A general procedure consists of splitting the plus fraction into certain carbon numbers and regrouping the split fractions into certain pseudocomponents. 
The results of a laboratory PVT study which had been conducted on a re-constituted original reservoir fluid were available. The laboratory measurements provided dew point pressure, gas produced, liquid dropout, gas deviation factor, and compositional information at various pre-determined pressure steps. However, the extended compositional analysis of the original reservoir fluid was not available. A method proposed by (Ahmed et al 1985) for characterizing $\mathrm{C} 7+$ was utilized to estimate extended compositional analysis for the original reservoir fluid. The extended compositional analysis was then used as input for PREOS phase behavior package to simulate the laboratory study.

\section{Comparison with Primary Production History}

Data on gas and liquid production at various measured reservoir pressures was available. The dry gas and liquid condensate production data were utilized to determine cumulative wet gas production and liquid yield at various reservoir pressures. The measured reservoir pressures were used in conjunction with the PR-EOS phase behavior package to simulate cumulative wet gas production and liquid yield at various pressures. The results were then used for comparison against available field data.

\section{Material Balance Study}

The first step in evaluating a potential reservoir for development is to perform a comprehensive material balance study. These studies typically involve using reservoir pressures along with gas and liquid primary production volumes. This data is used to estimate the initial hydrocarbons in place, characterize the fluids in place and estimate the reservoir size. If material balance solutions using the "tank model" approach cannot be validated then moving to more advanced reservoir simulation will be problematic.

A material balance study was conducted using two (2) phase $z$ gas deviation factors and cumulative wet gas production to determine the Initial Gas-in-Place (IGIP). The results were then utilized to estimate residue gas and liquid quantities at the conversion pressure. 


\section{Simulation of Storage}

Depleted gas or oil reservoirs after primary production are routinely used for gas storage. After refurbishing the facilities, natural gas is injected when demand for natural gas is low (summer time) and withdrawn when demand for natural gas is high (cold winter months). For dry gas fields, the withdrawn gas is normally dehydrated and sent to distribution systems. When depleted gas-condensate reservoirs are used for storage, the withdrawn gas will have a different composition than injected gas. This is due to the fact that retrograde liquid condenses out in the gas-condensate reservoir during the depletion process. This liquid is non-recoverable due to low liquid saturation in the reservoir. To prevent loss of retrograde liquid in gas-condensate reservoirs, gas cycling may be employed to displace rich gas phase, strip the liquids followed by re pressurization for gas injection in the reservoir to maintain its pressure. Alternatively, a depleted gas-condensate can be pressurized by gas injection to re-vaporize the retrograde liquid. Neither of these approaches is usually economically feasible due to high cost of gas and compression requirements. Therefore, most depleted gas-condensate reservoirs contain retrograde liquid at the conclusion of the primary production. Consequently, the gas withdrawn from a gas-condensate storage reservoir contains heavy hydrocarbons as a result of retrograde liquid evaporation. To prevent retrograde condensation in the gathering and transmission distribution systems, the withdrawn gas must be processed to remove these heavy hydrocarbons. The proper design of surface and processing facilities is essential in reliable operation of the gas-condensate storage fields. Significant volumes of liquid can be produced from the withdrawn gas, even with relatively low liquid yield, as results of high gas rates associated with storage operations.

In order to predict the composition of the reservoir fluid during storage cycles, the residue gas and liquid reservoir pressure were then completely mixed with pipeline gas in a proportion to return the reservoir pressure to discovery pressure. Finally, the performance of the reservoir for three (3) cycles of storage operations was simulated assuming $50 \%$ of gas in reservoir as top gas. It was thought that since the reservoir property affects were not considered in the initial tank model solution that the condensate yield predictions would be overly optimistic. This was thought not be a problem because the aim was to properly design the surface facilities to adequately handle the gas and liquid production. 


\section{COMPOSITIONAL MODEL DEVELOPEMENT}

Due to complex phase behavior, mixing, and reservoir rock-fluid interactions, it was necessary to utilize a compositional reservoir simulator to study the performance of the reservoir during storage operations. These type reservoir simulators are typically used for studies that require phase equilibrium models, such as, equation of state for oil and gas associate with gas cycling and recycling projects. The more complex compositional model uses the equation of state (EOS) model to determine the PVT (Pressure-Volume and Temperature) properties of oil and gas phases. The simulator then uses the tuned EOS equation of state model to determine the properties of the mixture of components for certain reservoir conditions. The compositional models differ in this respect from black oil models where the composition of the hydrocarbons is held constant.

It should be noted that significant volume of liquid can be produced from the withdrawn gas even though the liquid yield during storage withdrawal cycle is significantly lower than the liquid yield during the primary production. This mainly due to high gas withdrawal rates during storage operation. Consequently, the proper design of the surface and processing facilities to handle the produced liquid is essential for the reliable operation of the storage field. The condensate yield during storage withdrawal cycle is the key parameters for the storage facility design and operation. The condensate yield depends on the composition of the withdrawn gas from the storage. The degree of mixing among residue gas, evaporated condensate, and the injected gas determines the withdrawn gas composition. The degree of mixing will depend on a number of factor including the well configuration, residence time, reservoir structure, and injection-withdrawal schedule. To accurately evaluate the degree of mixing a compositional numerical reservoir simulator was employed in this study.

The next step involved developing the compositional numerical reservoir simulator for use in evaluating the effects of the reservoir properties on the degree of mixing at reservoir conditions. The use of compositional simulators requires an accurate fluid characterization to initialize the beginning state of the model. An Equation of State (EOS) Model for the multicomponent system must be used in conjunction with the reservoir simulator. 
After the compositional model was developed, the objective of this part of the study was to analyze the results of the first storage cycle and gain better understanding of the various processes that influence condensate production during storage operations in order to operate and manage the storage field efficiently. As was indicated earlier, the degree of mixing depends on a number of factors including the well configuration, residence time, reservoir structure, and injection-withdrawal schedule/patterns determines the compositional changes in the withdrawn storage gas and the condensate yields. The ability to develop a reliable prediction of the condensate yield during storage withdrawal cycles would help in the effectively designing the storage surface and treatment facilities for storage operations.

The degree of mixing among residue gas, evaporated condensate and the injected gas would determine the withdrawn gas composition and the condensate yield. The degree of mixing depends on a number of factor including the well configuration, residence time, reservoir structure, and injection-withdrawal schedule. To accurately evaluate the degree of mixing a compositional numerical reservoir simulator was employed. The reservoir simulation software used in this study was GEM(Generalized Equation-of-State Model) Reservoir Simulator developed by the Computer Modeling Group (CMG). GEM is a robust, general equation-of- state (EOS) based compositional simulator that can model any type of reservoir where the fluid composition and their interactions are essential to the recovery process. GEM can effectively model complicated phase behavior interactions such as gas condensate storage reservoir and provides extensive well management options and a flexible set of surface facilities. A key component of GEM is WinProp that can be used to create the complete PVT data fluid characterization. The simulation study consisted of four(4) steps that will be discussed below:

1. Reservoir Fluid Phase Behavior Prediction

2. Simulation of Primary Production

3. Simulation of Storage Performance with Vertical Wells

4. Simulation of Storage Performance with Horizontal Wells

5. Storage Operational Design Evaluation

6. Impact of Reservoir and Fluid Properties 


\section{Reservoir Fluid Phase Behavior Prediction}

To define the fluid properties at the reservoir and surface conditions, usually a laboratory PVT analysis, such as constant volume depletion (CVD) test, is performed on the original reservoir fluid. The results of a laboratory PVT study performed on the original reservoir fluid for the reservoir under study were available. The laboratory measurements provided dew point pressure, gas produced, liquid dropout, and 2-phase deviation factor at a series of pressures. The extended compositional analysis for the original reservoir fluid however was not available. An extended compositional analysis for the original reservoir fluid was estimated by obtaining reasonable agreements with the laboratory measurements. Subsequently, the available pressure and wet gas production data and 2-phase deviation factors were utilized to perform a material balance study to determine the Initial Gas-inPlace (IGIP).

In order to use the compositional reservoir simulator, GEM, it is necessary to first generate the phase behavior prediction model for the reservoir fluid. WinProp is an EOS-based phase behavior simulator that is incorporated in GEM for this purpose. WinProp can split the heavy end (C7+ fraction) into a number of pseudo components and has a flexible component lumping option. WinProp also has a robust adaptive regression algorithm for tuning the EOS parameters to match laboratory PVT data. The estimated extended original fluid compositional analysis and the results of the laboratory PVT study were used as inputs to WinProp. The predicted phase behavior utilizing component lumping and the regression analysis provided similar results as those previously obtained by PR-EOS phase behavior simulation package. This confirmed the reliability of the estimated extended compositional analysis and also provided the necessary phase behavior input for GEM compositional model. 


\section{Simulation of Primary Production}

A reliable model of the reservoir was necessary to predict the degree of fluid mixing and storage performance. Detailed reservoir description obtained from geological data, seismic map, and core and well log data were utilized to develop a 3-dimensional model of the reservoir. There are only three (3) wells in the reservoir; one production well, one observation well and one dry hole. As a result, the available core and log data were very limited. The core-log data were utilized to develop a correlation for permeability distribution prediction. The pore volume of the reservoir was adjusted to match initial gas in place (IGIP) obtained in previous work from the material balance calculations.

In Figure 3.2 a Two (2) dimensional representation of the grid spacing with the Net Pay grid property shown. This help to visualize the reservoir structure as interpreted from the geologic characterization. The Three (3) dimensional grid map in Figure $\mathbf{3 . 3}$ illustrates the reservoir model used in this study with original production well location and recently drilled observation well. In Figure $\mathbf{3 . 4}$ the Cross Section through the main part of center of the well defined structure shows the Grid Pay Depth.

Net Pay (ft) 1979-05-01 Klayer: 1

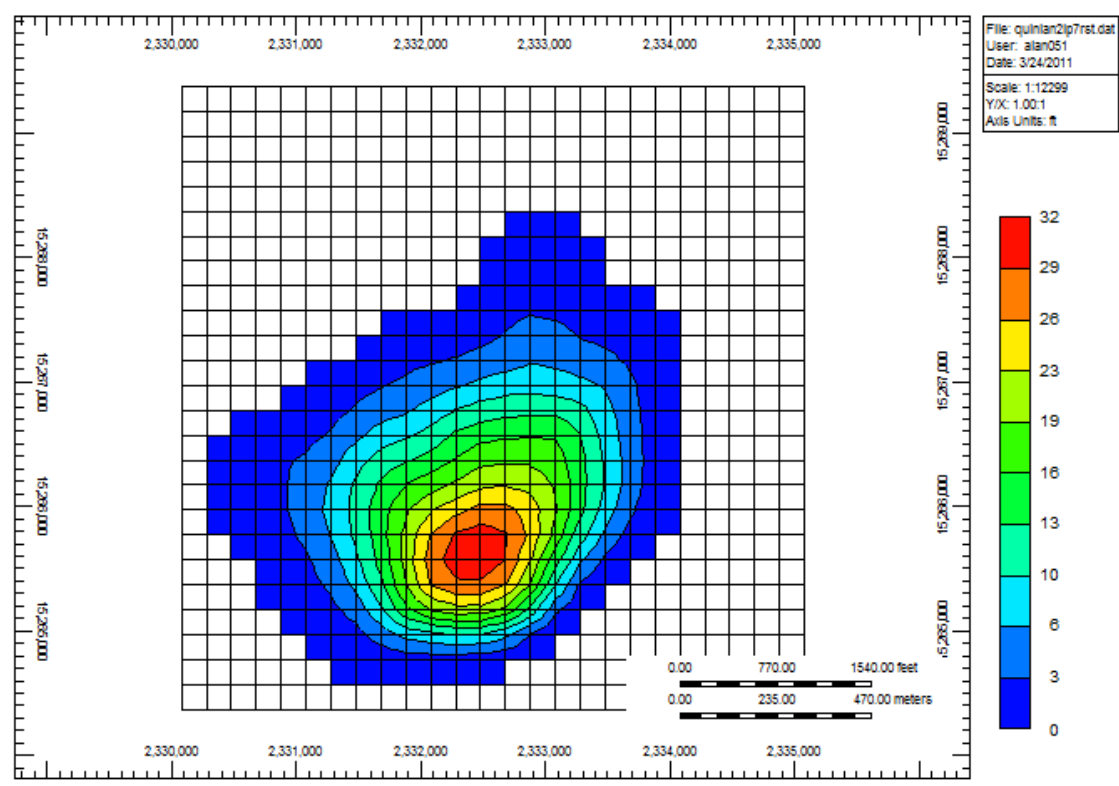

Figure 3.2. Two(2) dimensional grid map showing Net Pay of formation 
Grid Top (ft) 1979-05-01

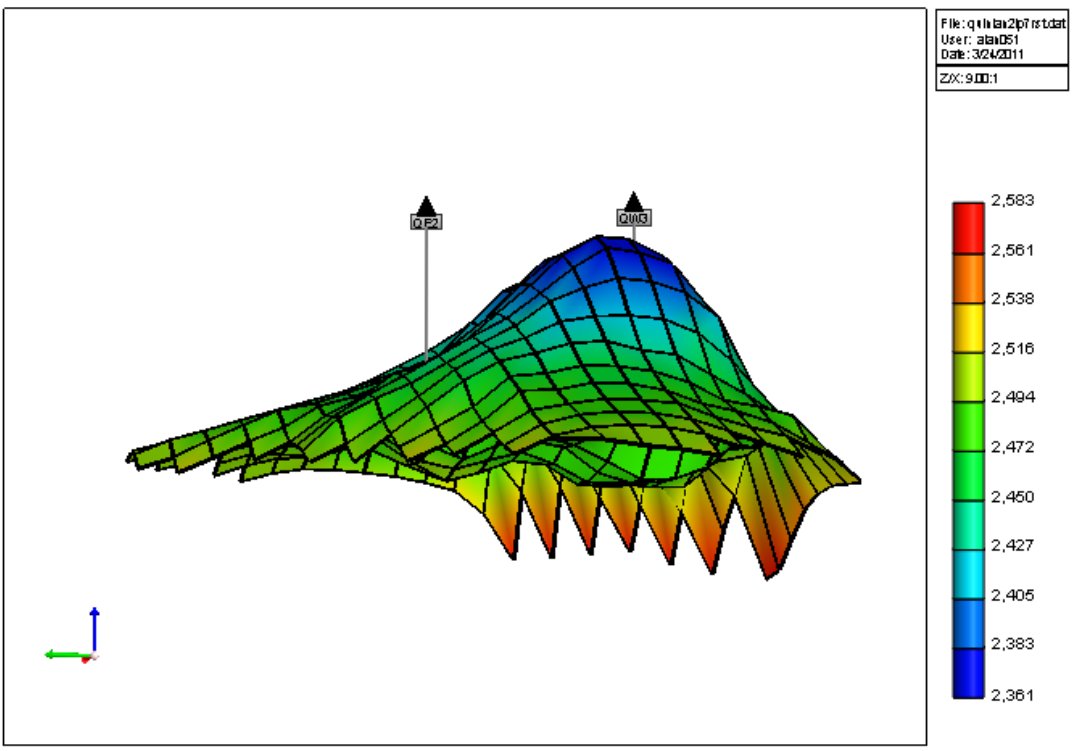

Figure 3.3. Three(3) dimensional grid map showing Grid Top of formation

Grid Paydepth (ft) 1979-05-01

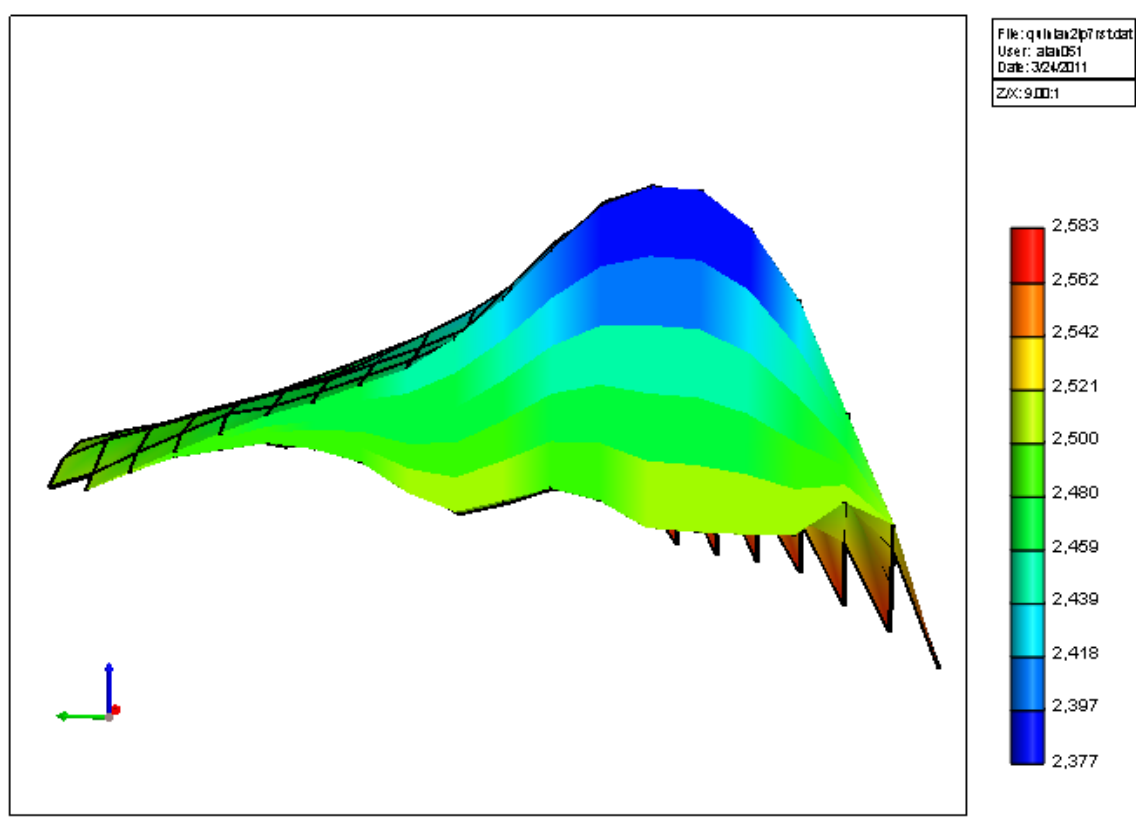

Figure 3.4. Cross Section of structure showing Depth of Pay 
The model was then produced by assigning a constant wellhead (separator) pressure similar to actual field operations. Later in the production history the reservoir pressure had declined to below the sales line pressure resulting in declining production volumes.

An 85 HP compressor was installed to lower the wellhead pressure and increase the production volumes. This was easily implemented with GEM because of the flexibility of modeling multiple surface plant separators and stages. Another strong point of GEM is the ability to optimize separator conditions for maximum liquid recovery.

The permeability prediction correlation had to be slightly modified to match the dry gas production during the primary depletion period. The table below Table 3.1 shows the CMG output results from the history matching versus the actual production and pressure data from available historical data.

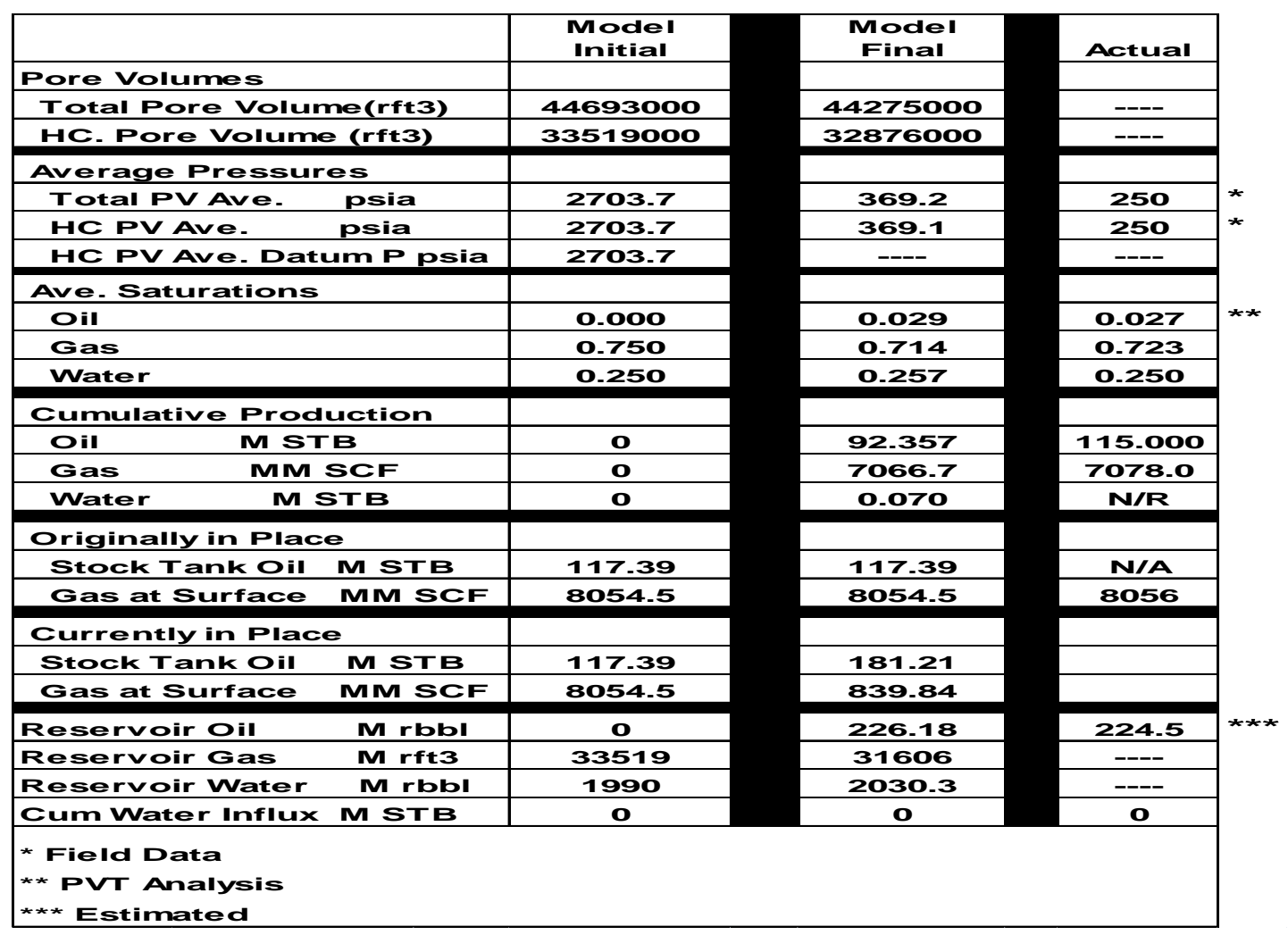

Table 3.1. Comparison of Model Output versus Actual Field Data 
Table 3.1 compares the predicted liquid yields by the EOS, the simulator, and the field data. The results clearly indicated that the predictions by the reservoir simulator are superior to EOS predictions. The reservoir simulator accounts for rock-fluid interaction that EOS, which is basically a tank model, cannot account. The close match between the simulator results and observed production performance provided the confidence in the model to be used for storage predictions. For reference the Initial model data is resented that shows that the reservoir was at the saturation pressure with no liquid saturation

\section{Simulation of Storage Performance with Vertical Wells}

The model developed in the previous section was utilized to predict the performance of the reservoir during storage operations. The restart option was utilized to initiate the simulation at the conclusion of the primary depletion and after a satisfactory history match had been obtained. Lean pipeline gas was injected into the reservoir utilizing six (6) new injection/withdrawal (I/W) wells. Figure $\mathbf{3 . 5}$ is the 3- dimensional grid map of the reservoir model with original and new well locations. The reservoir was pressurized back to the discovery pressure using a constant injection rate over a period of 4 months (April-August). The wells were then shut-in for 3 months (August-November). Subsequently, the wells were produced at constant rate to simulate the withdrawal cycle. A second injection-withdrawal cycle was also simulated.

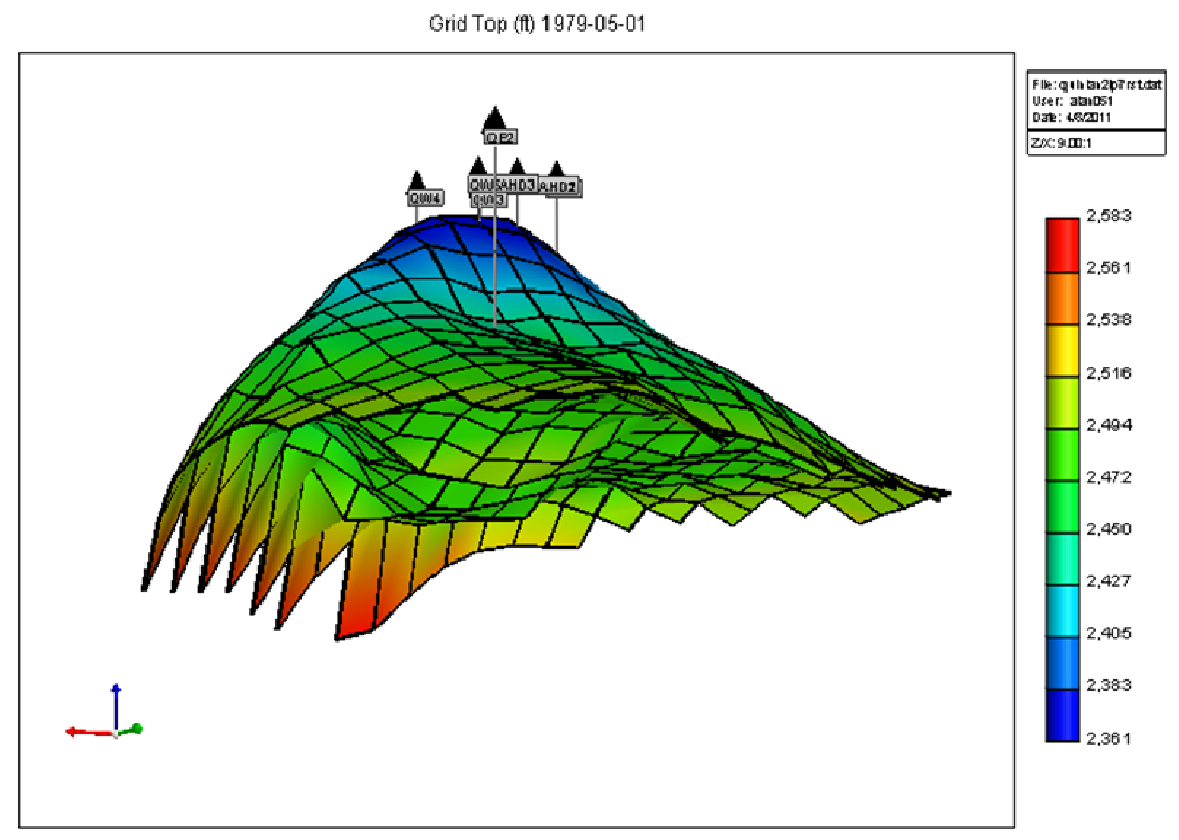

Figure 3.5. Three(3) dimensional grid map showing New Well Locations 


\section{Simulation of Storage Performance with Horizontal Wells}

The model developed for primary performance simulation was utilized to predict the performance of the reservoir during storage operation. The restart option was utilized to initiate the simulation at the conclusion of the primary depletion. Pipeline gas was injected into the reservoir utilizing four (4) new lateral/horizontal injection/withdrawal (I/W) wells. The lateral/horizontal wells were considered because of the reservoir's limited surface access. The two existing vertical wells in the reservoir were used as observation wells. The injection and withdrawal scheme used in the previous section was also implemented here. In addition, a constant pressure withdrawal case was also simulated.

One of the wells has two multilateral that had to be represented in the well trajectory recurrent data section of the software. The two existing wells in the reservoir were used as observation wells.

The final step to describe in the methodology was to evaluate several storage designs and scenarios and study how these impact condensate yields during storage operations. This will enable operations to efficiently manage the storage field. Changes at the reservoir level as well as changes to surface facility would be evaluated depending on what engineering solutions were required. The ability to continue storage history matching based on actual operating data facilitated this part of the study.

\section{Storage Operational Design Evaluation}

The objective of this part of the study was also to analyze the results of the first storage cycle so a better understanding of the various processes that influence condensate production during storage operation in order to operate and manage the storage field efficiently. As was indicated earlier, degree of mixing which depends on a number of factors including the well configuration, residence time, reservoir structure, and injection-withdrawal schedule determines the compositional changes in the withdrawn gas and the condensate yields. Due to complex phase behavior, mixing, and reservoir rock-fluid interactions, it was necessary to utilize a compositional reservoir simulator to study the performance of the reservoir during storage operations. The reservoir model that had been developed in our previous steps was utilized for analyzing the results of the first storage cycle. The rate 
schedules were adjusted to reflect certain low and high constant rate designs along with periods of high peaking demands. It was thought that the liquid condensate yields might be affected by increased rates in the peaking designs.

When the design for the storage project was being evaluated the surface facilities were not designed to operate as an efficient extraction plant for removing fluids, specifically hydrocarbon fluids. Generally when a gas condensate field is being developed considerable amount time and money may be needed to capture the valuable liquids condensed from the gas streams. However, when a storage facility is being designed the most important factors are capacity and deliverability designs. The condensate associated with storage operations are generally considered a waste stream depending on the quality, quantity and volumes. Depending on the revenue generated from the sale of the condensate it can be marginal on whether the volumes should be minimized or maximized. These factors can enter into the decisions to design expensive surface facilities capable of adequately capturing the maximum amount of the liquids. If it is not profitable to remove these liquids might better be left in the gas stream in the form heavier hydrocarbons and richer gas.

Another objective of these studies was to evaluate certain surface facilities and their affect on condensate recovery and sales outlet gas streams. Understanding that the surface facilities required for storage operations will not operate as efficiently as in production operations, gas and liquid recovery are still paramount.

\section{Impact of Reservoir and Fluid Properties}

After the compositional reservoir simulator was successfully used to predict the performance of a gas condensate reservoir the model was used to evaluate fluid property changes as a result of mixing of the solvent gas with the wet residue gas. These changes were first applied to the reservoir characterization to see how this affected the condensate yields predicted from the model. 
The following reservoir simulation cases were developed, executed and analyzed to address the problems described above with storage operations design, operational changes and the reservoir affects on condensate yields:

1) The location of the original production well was changed to see how this affected the primary production history match. As has been stated in early discussion the original well location was located near the outer boundary flank of the reservoir structure.

2) The affects of changing the permeability distribution of Top two(2) Layers of the reservoir model on the Original History Match. This was done in attempt to explain why condensate yield predictions were deviating from actual storage operating schemes

3) The affects of changing the Permeability distribution from Top two(2) layers to Bottom two(2) layers. Again this was done in attempt to explain why condensate yield predictions were deviating from actual storage operating schemes

4) To evaluate the wellbore effects on condensate blockage or Non Darcy flow effects and how these Skin Factors influence condensate yields. There has been a great amount of literature on this subject describing its importance.

5) High rate or Peaking Tests were modeled to evaluate condensate yield recoveries. High rates from storage facilities are normal operations and must be fully understood particularly where problems might interfere with the ability to meet the requirement demands of storage service.

6) Measure the affects of changing the Permeability distributions on Storage Scenario runs. This was done to explain the affect on condensate yields and evaluate the impact on deliverability.

7) Simulate Realistic Surface Conditions for Condensate Yield Calibration. As mentioned earlier the surface separation facilities were not designed with high efficiency in mind.

8) Study the wellbore effects on condensate yields and determine advantages and disadvantages of utilizing vertical well and horizontal well solutions 


\section{CHAPTER 4 RESERVOIR MODEL DEVELOPMENT}

\section{Reservoir History}

The Quinlan Reef production field was discovered in 1977 and located near Olean, NY (Figure 4.1). The Quinlan E-2(QE2) well was drilled based on $2 D$ seismic data indicating a reef build-up on December 31, 1977 at a depth of 4332 feet. Due to unexpected flows of natural gas only the top few feet of the reef was penetrated with the QE-2. The only well drilled (QE2) in the pool drained the entire acreage over the life of the field. QE2 had an original production rate of 2.53.0 MMSCFD and approximately 100 BPD of condensate. The original shut in reservoir pressure was estimated at 2710 psi. The well blew out while drilling due to the high gas volumes encountered in the top of the reef structure. The well just nipped the northeastern part of the structure

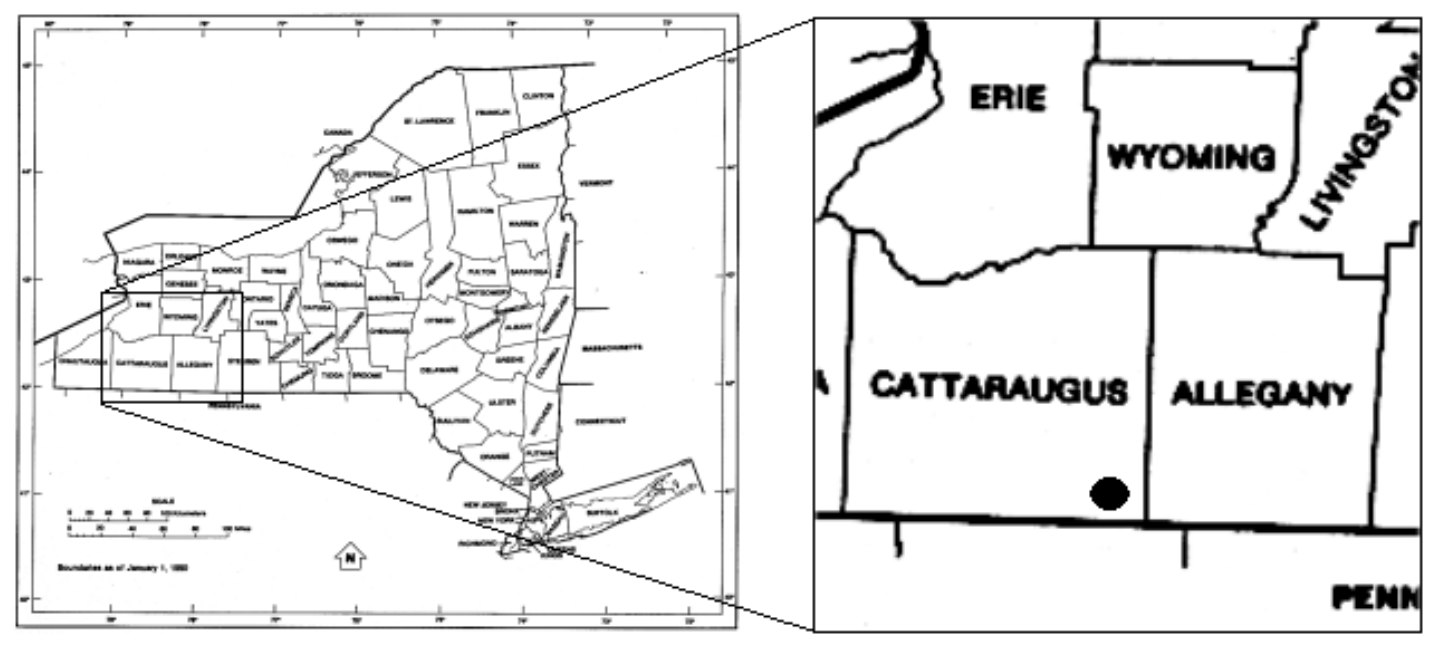

Figure 4.1. Quinlan Field Geographic Location

The field has been classified as a cold retrograde condensate reservoir with a bottom hole temperature estimated at $120 \mathrm{~F}$. Most condensate reservoirs have temperatures in excess of $200 \mathrm{~F}$. This phenomenon causes the liquid drop out in the reservoir to be higher in colder reservoir temperatures thus reducing the amount recoverable liquids. An earlier PVT Constant Volume depletion study estimated a recovery of $32 \%$ based on recombined separator liquid products. There is relatively no water production associated with the Quinlan field thus the reservoir exhibits no water drive effects. The reservoir fluid properties are much leaner than rich gas condensate fluids increasing the recovery and lowering the percentage amount of condensate dropping out in the reservoir. However, the low oil saturation decreases any mobility and prevents any flow of the fluid in the pore spaces. 
In January 1978 the initial shut in stabilized tubing pressure was recorded to be 2317 psia that equated to a bottomhole reservoir pressure of 2710 psia. Initial gas production began in May 1979 at a rate of 3.000 MMSCFD with an initial condensate oil production rate of 100 BBLD. This corresponded to a condensate gas ratio (CGR) of approximately 33 BBLS/MM.

The reservoir pressure had declined from 2710 psia to 588 psia in December 1995. There was a period from 1996 to 1998 where production was shut in due to ownership changes and renegotiation of sale agreements. Also, in August 199885 HP of compression was installed due to the flowing top hole pressure had equalized with the existing sales line pressure. Figure $\mathbf{4 . 2}$ and Figure 4.3 show the monthly and cumulative gas production from the field from 1978 to 2005.

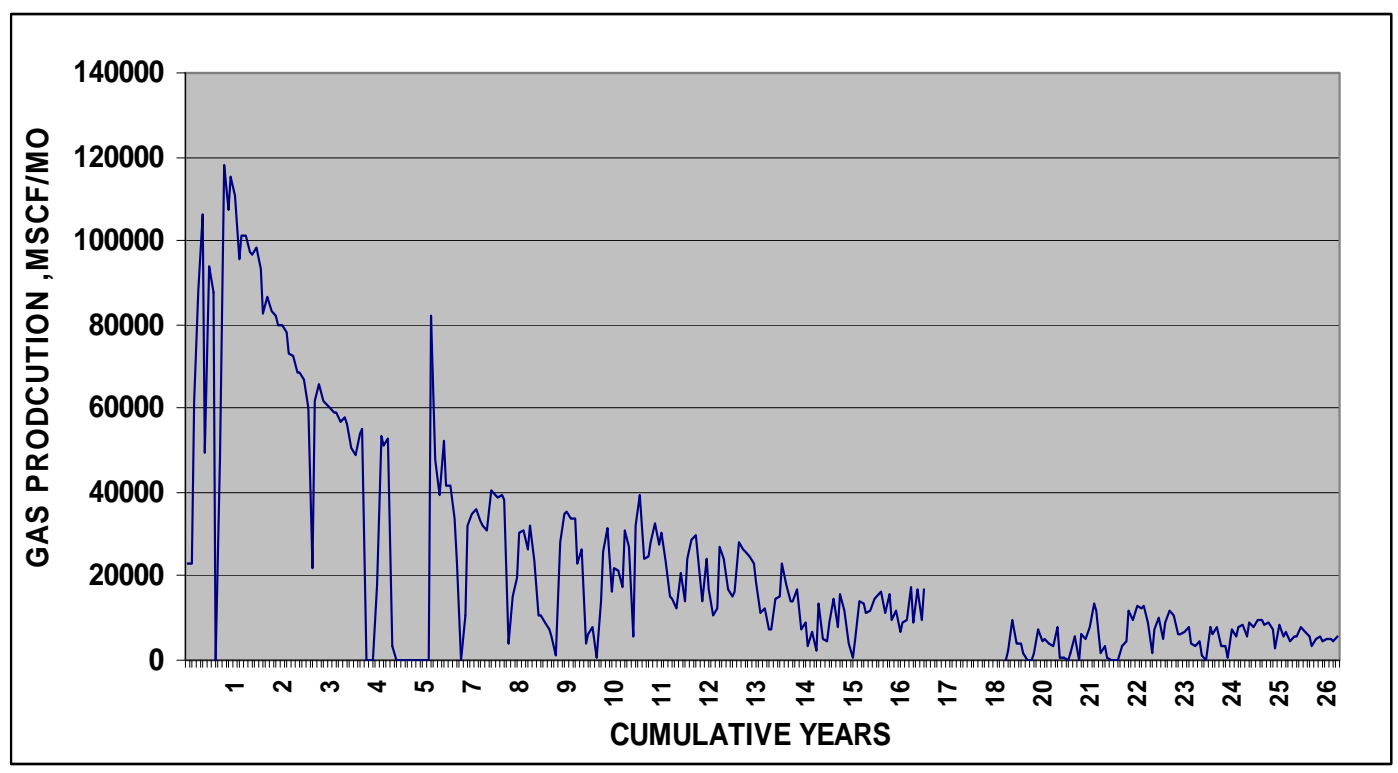

Figure 4.2. Monthly Gas production from Quinlan field 1978-2005 


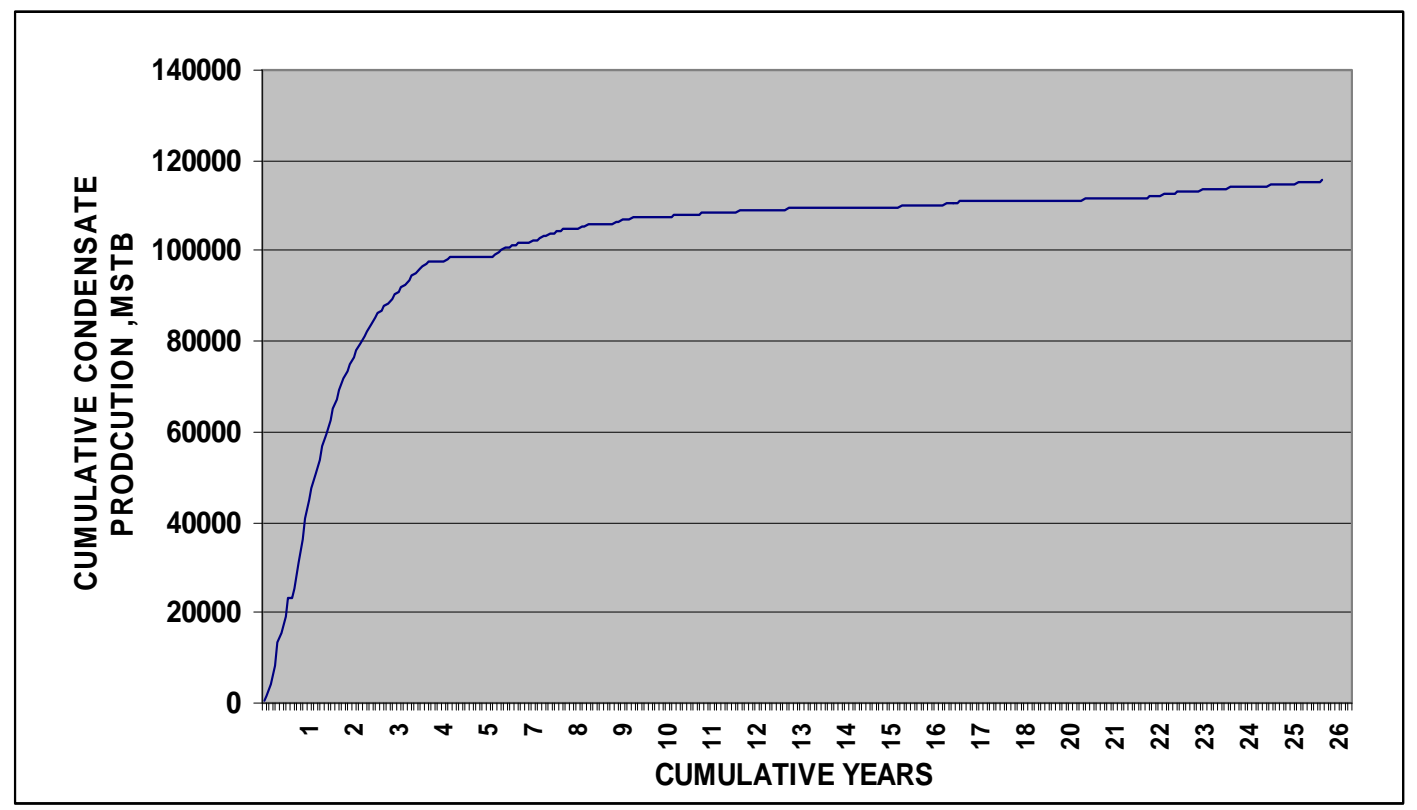

Figure 4.3. Cumulative Gas production from Quinlan field 1978-2005

Figure 4.4 and Figure 4.5 show the monthly and cumulative gas production from the field from 1978 to 2005.

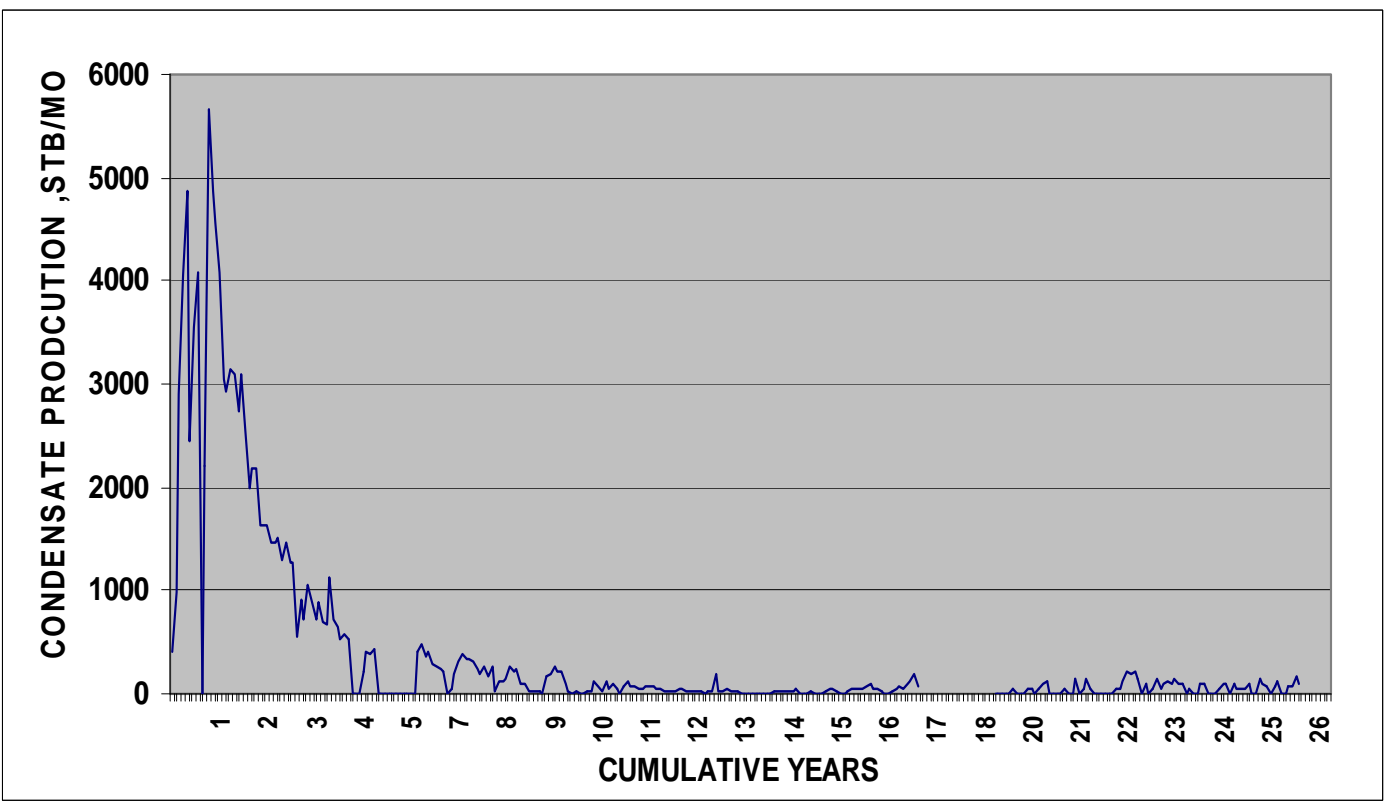

Figure 4.4. Monthly Condensate production from Quinlan field 1978-2005 


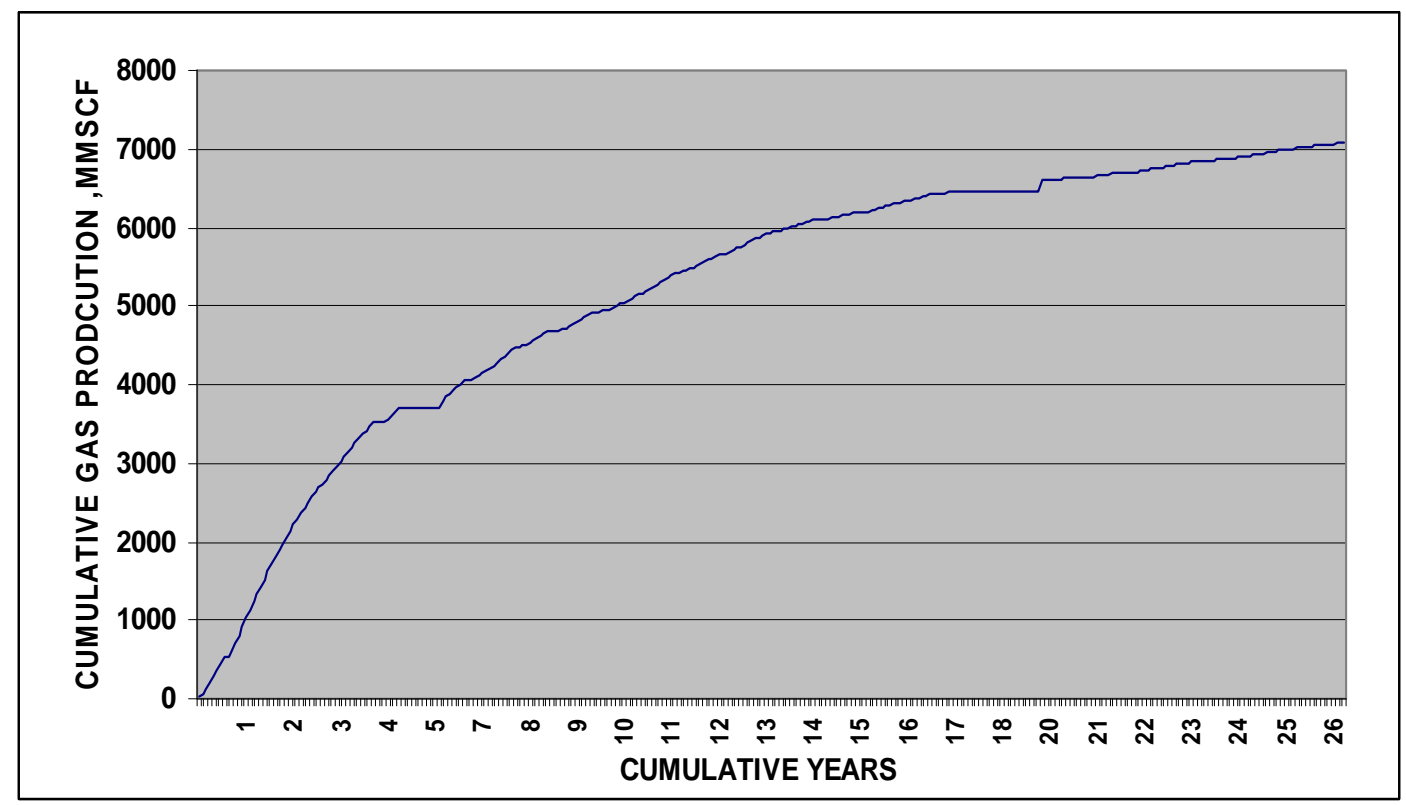

Figure 4.5. Cumulative Condensate production from Quinlan field 1978-2005

As of August 2005 the total wet gas production was 7.078 BCF and the cumulative monthly liquid production history shows that approximately 115,007 bbls total condensate has been recovered. The results of the original PVT fluid study, the recent Equation of State study and recent compositional reservoir simulation study all indicate that approximately one third of the original condensate liquid in place has been produced. It estimated that there between 180,000 and 230,000 remaining stock tank barrels in the reservoir.

There was insufficient data to make an estimate of the volumetric initial gas in place because the only well drilled QE-2 only penetrated the top 20 feet making it difficult to estimate the reservoir pay thickness. There were some estimates of the gross pay thickness based on the earlier seismic data. 


\section{Geologic Description}

Onondaga Limestone (Middle Devonian geologic age) occurs in southern New York state and Northern Pennsylvania(see Figure 4.1). The reef in question is a typical bioherm covering an area of approximately 221 acres with an estimated thickness of 190 feet at a depth of 4500 feet. These bioherms became extinct submarine knolls and where left to be covered later by the Marcellus shales. These reefs present good developed vertical and lateral permeability.

The cross section below in Figure 4.6 illustrates the reef platform and the overlying strata consisting of many shales and shaley sands including the Marcellus shale. Also the cross section shows the typical three part reef structure made up starting from the bottom, the reef platform, the lower reef core facies and the upper reef core facies. The porosity is limited to the upper reef core facies. The reef build up is thought to have been a patch reef absent any adjacent shelf material contrary to barrier reef construction.

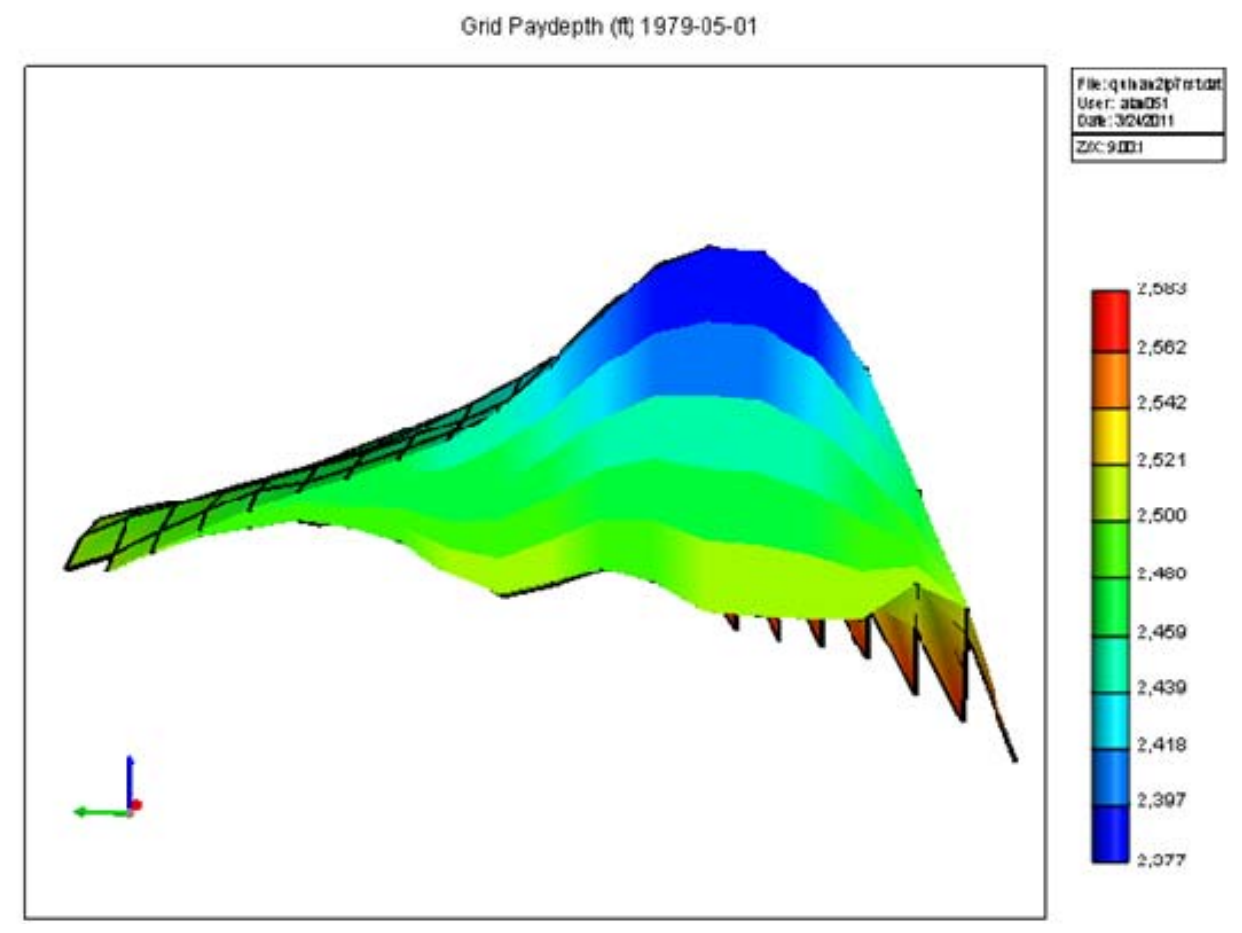

Figure 4.6. Onondaga Reef Cross Section 


\section{Early Seismic Work}

There was focused interest in the area after the QE-2 Middle Devonian Onondaga reef well was discovered by Pennzoil in 1978. There was speculation on where QE-2 was located on the Reef structure. In 1979 a two dimensional seismic survey (ESI 12-79) was shot from a southwest to northeast orientation that showed that QE-2 was located on the northeast flank of the reef and to the south and southwest there were more extensive buildups of reef structure. Two (2) more two dimensional seismic lines (ESI 16-81) and (ESI 15-83) were shot in 1981 and 1983. See Figure 4.7 for the two dimensional seismic lines acquired from Pennzoil. Figure 4.8 shows the structure interpretation.

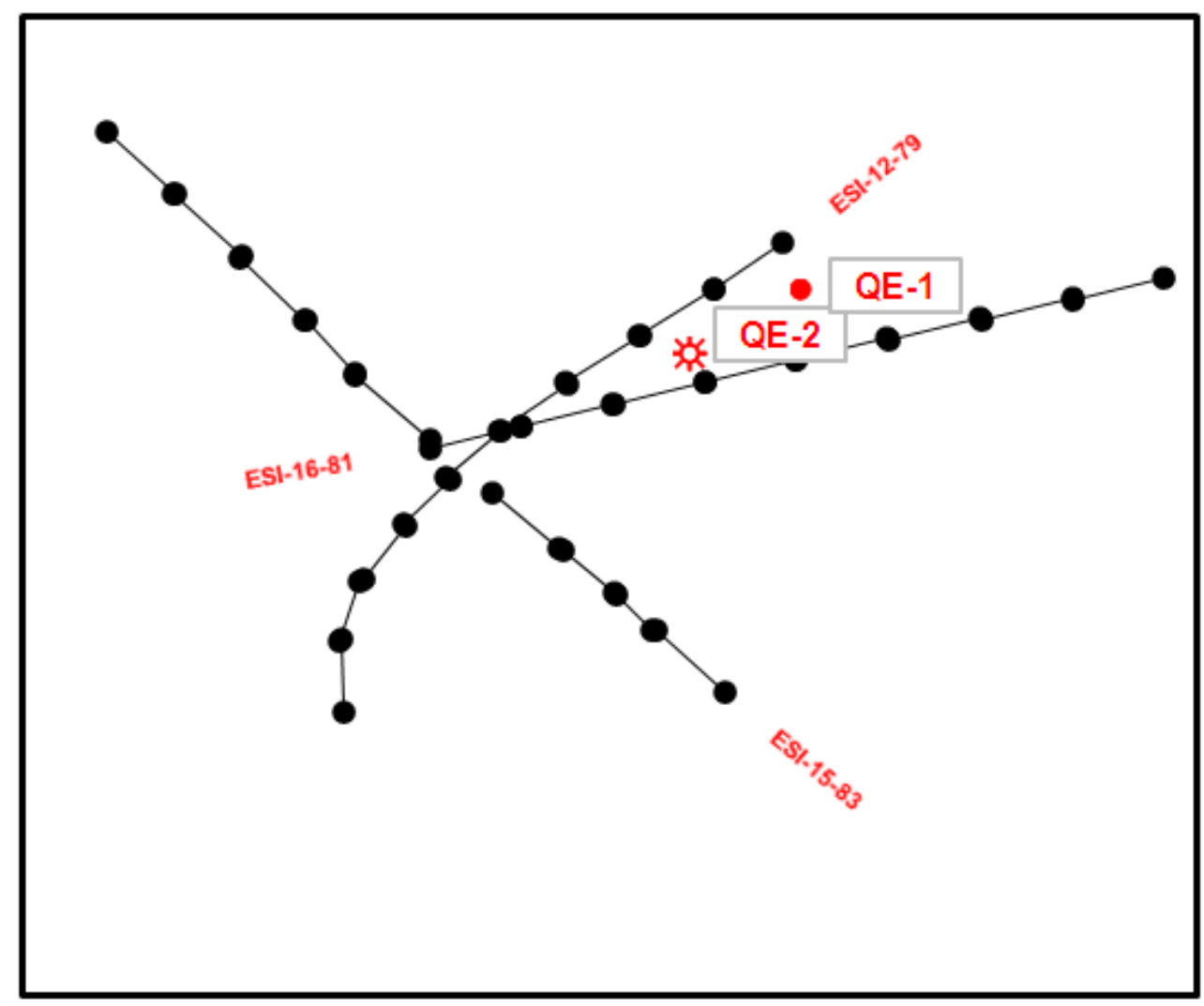

Figure 4.7. 2D Seismic Lines $(1979,1981$, and \& 1983) 


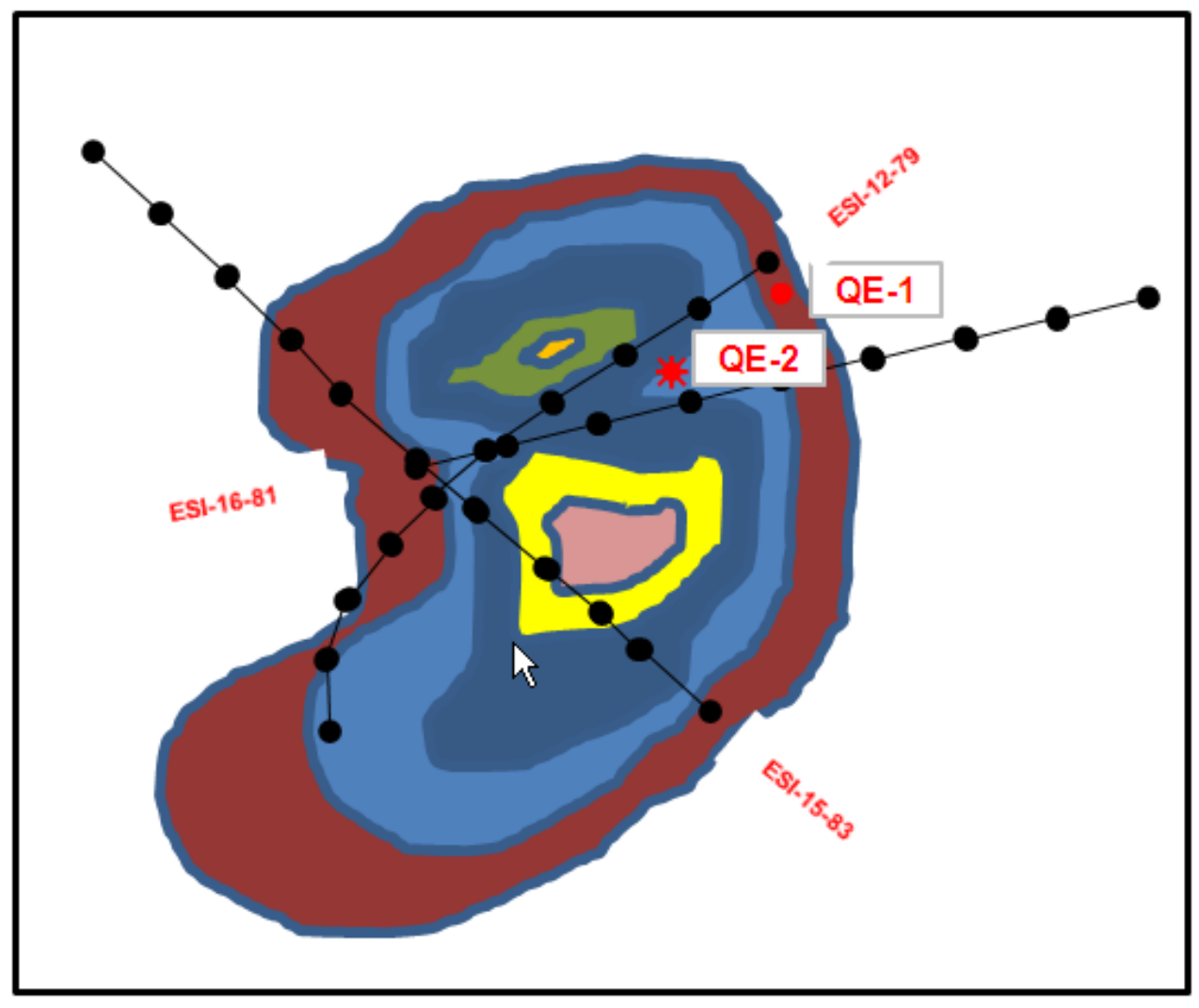

Figure 4.8. 2D Seismic Interpretation(1979, 1981, and \& 1983)

In 1998 it was proposed to shoot three dimensional (3D) seismic over the entire prospect to further delineate the reservoir boundary limits. This proposal was based on decision to develop the reservoir for storage. This would be a 4.642 square mile, three-dimensional (3D) seismic survey is shown in Figure 4.9. Some of the objectives justifying the 3D seismic project were:

1) determine the reservoir boundary limits

2) accurate reservoir property characterization

3) select the best place to drill and core a test well

4) help in selecting a target for the directional drilling of development wells 


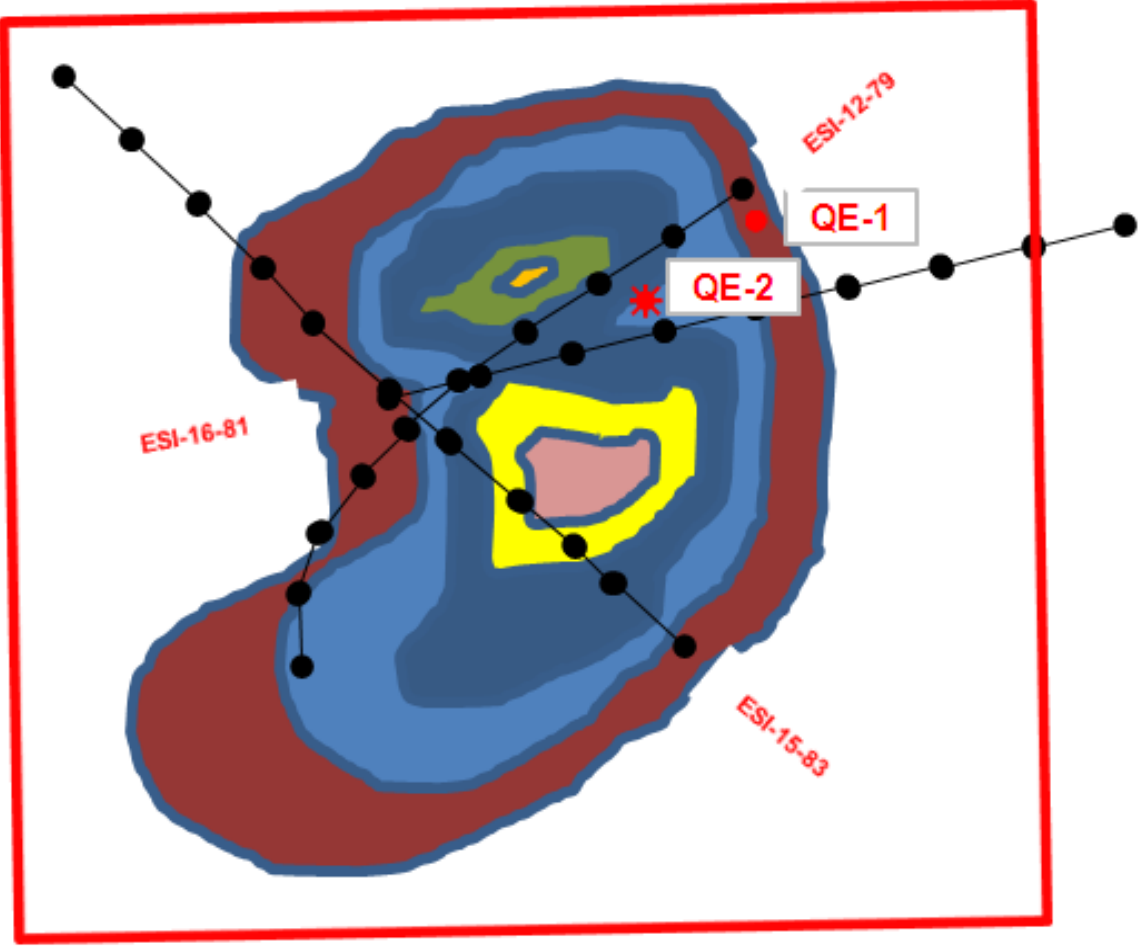

Figure 4.9. 3D seismic proposed areal grid(1998 proposed)

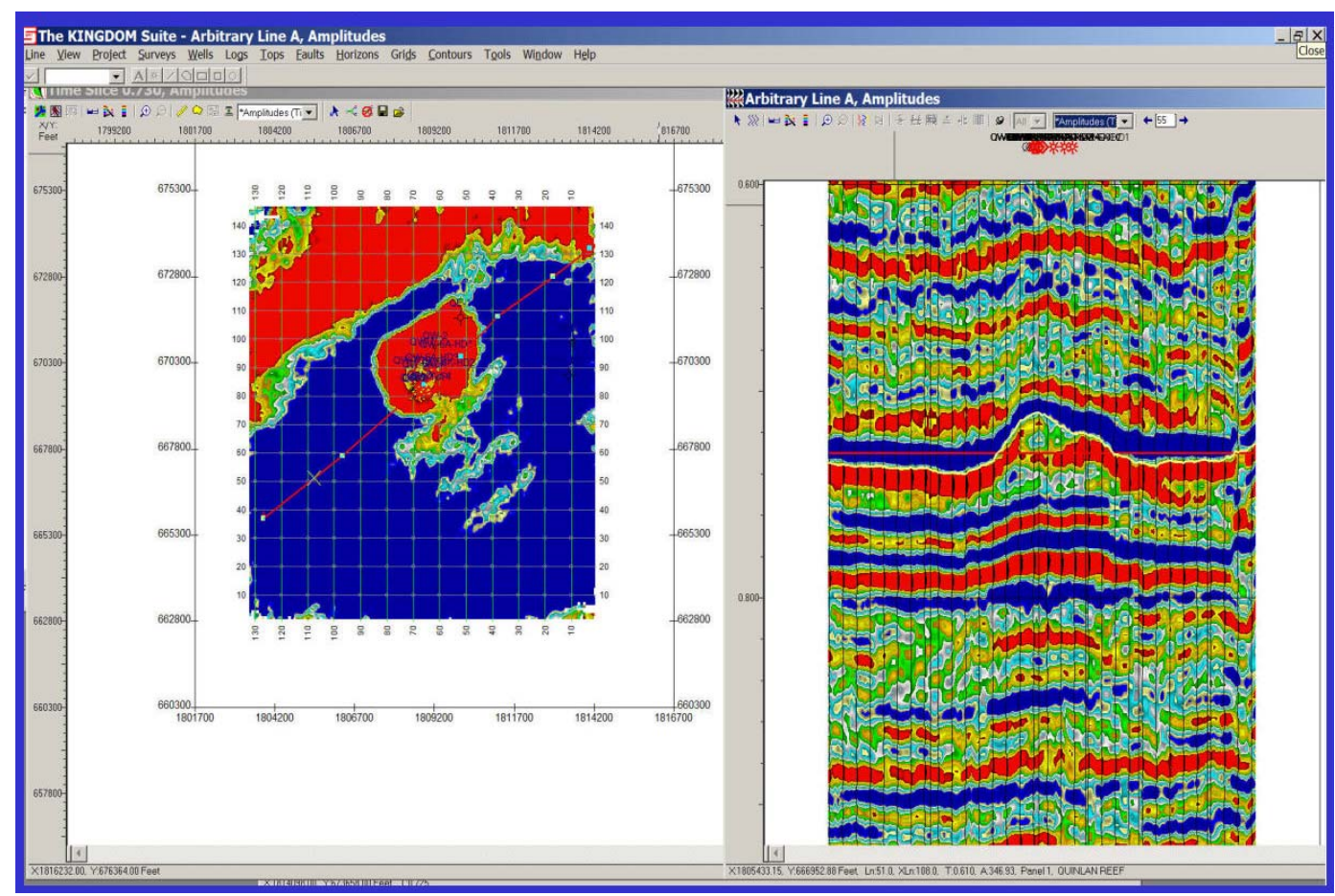

Figure 4.10. Three(3D) $730 \mathrm{~ms}$ Time Slice Structure Onondaga Formation 


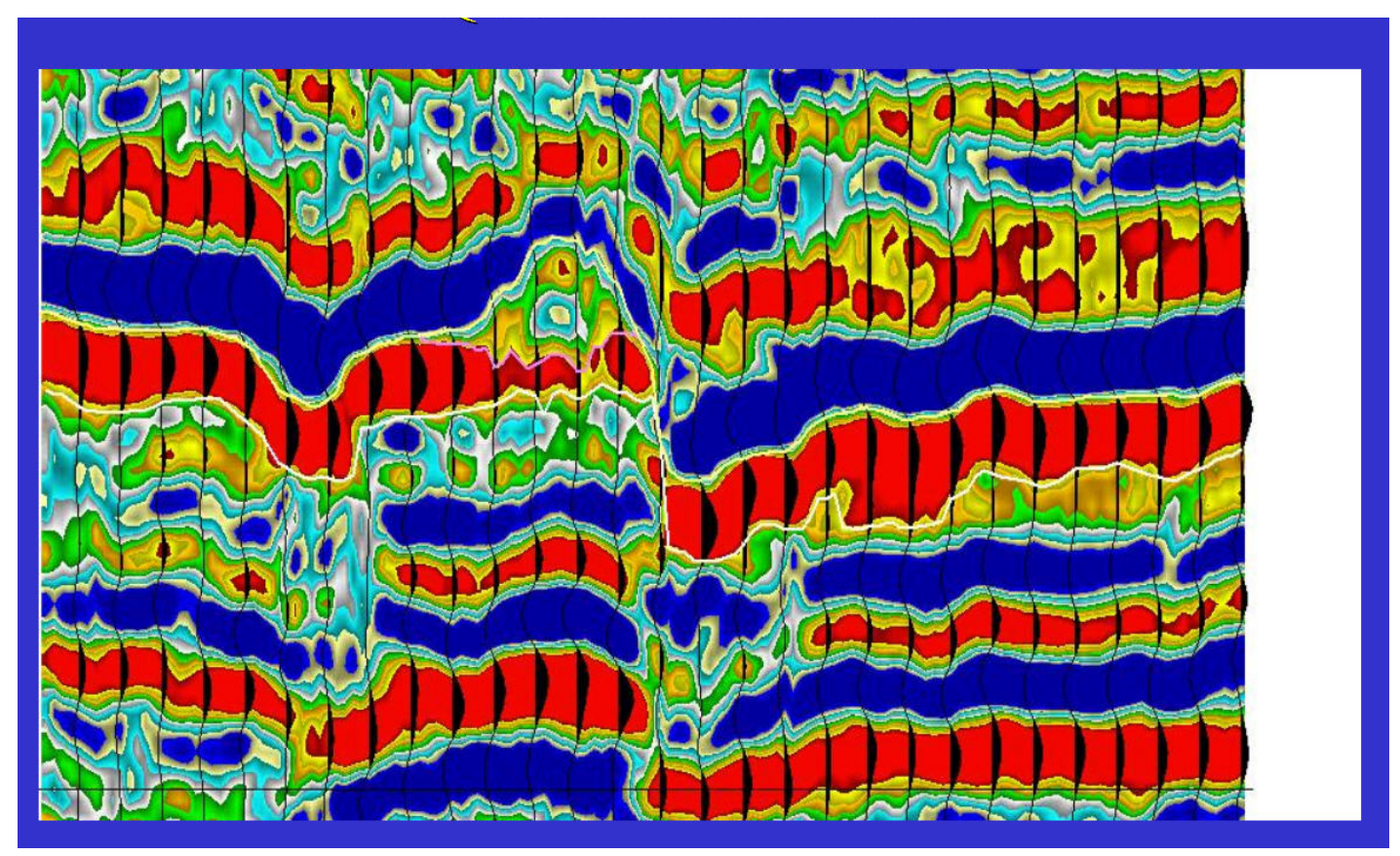

Figure 4.11. Three(3D) Time Slice of Onondaga Formation

After the 3D Seismic was interpreted a second test well QW-3 was drilled in 1999. This well was approximately $1000 \mathrm{ft}$ southwest of the original production well QE-2. The primary goal of this well was to core the Onondaga interval, obtain an openhole logging suite and complete the well so it could be welltested and used for observation of reservoir pressure. See Figures 4.10 and 4.11 for the 3D seismic interpretation.

Core analysis(Figures 4.12 and 4.13) indicated that the Onondaga formation comprising the Quinlan reef is mainly homogeneous in structure and composition. The framework is made up of calcium carbonate from coral and echinoderm skeletal fragments. Acid solubility tests indicated an increase in porosity of between $9-23 \%$ of the original $9.1 \%$ average porosity. The core was analyzed using Thin Section analysis, Scanning Electron Microscopy(SEM), X-ray diffraction(XRD) , Whole core, Standard Core plug, Acid solubility analysis, Computed Tomography and Geomechanical Analysis. 


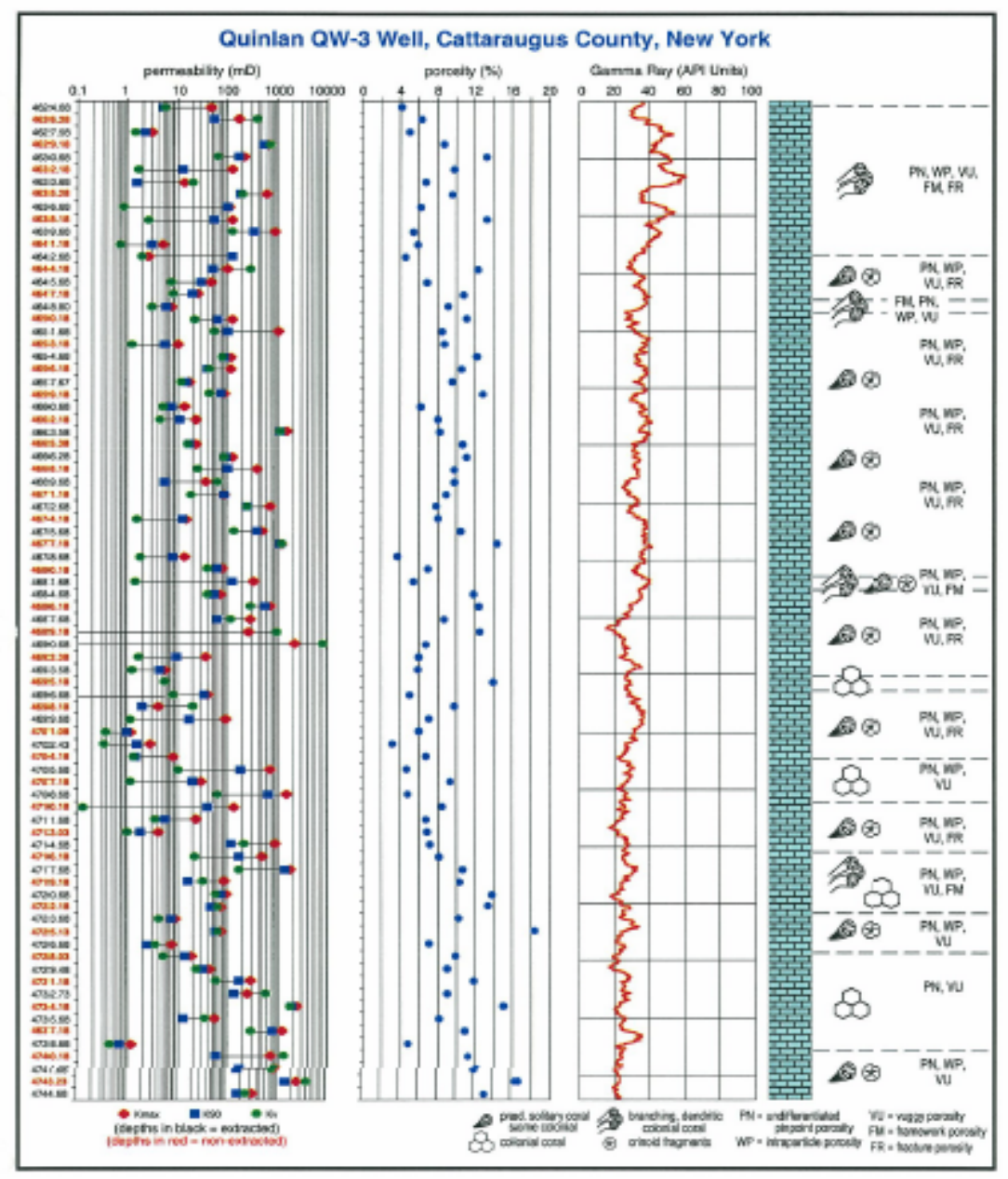

Figure 4.12. Summary Core Permeabiltiy, Porosity, Gamma Ray and Lithologic Description for the Onondoga Formation(Westport Geological Services)

The depositional environment appears to have been an in situ solitary and colonial coral buildup. The coral fragments are bound together with bioclastic material and calcite with silt and clay filling the areas between the corallites. There are some colonial Favosites and phaceloid Corallites with void spaces throughout most of the core. Some of the Corallites are roughly equal in size and evenly distributed across the colony surface. The core analysis indicated a porosity ranging from $3.2 \%$ to $18.4 \%$ with an average of $9.1 \%$. The porosity has been enhanced by dissolution of the reef material forming vuggy pores. The horizontal permeability ranged from 0 to greater than 2 darcies and vertical permeability ranged from less than a millidarcy to 8 darcies. The permeability to porosity relationships were relatively linear. The higher vertical permeabilties(K90) were isolated to only a few feet of the whole core with the majority of the higher permeability areas located in the horizontal plane(Kmax). 
Table A2.2: Whole Core Analyses, Extracted Samples

\begin{tabular}{|c|c|c|c|c|c|c|c|c|c|c|}
\hline \multirow{2}{*}{$\begin{array}{c}\text { Sample } \\
\text { No. }\end{array}$} & \multirow{2}{*}{$\begin{array}{l}\text { Top } \\
\text { Depth } \\
\text { (teet) } \\
\end{array}$} & \multicolumn{3}{|c|}{$\begin{array}{l}\text { Permeability } \\
\text { (mD) }\end{array}$} & \multirow{2}{*}{$\begin{array}{c}\text { Porosity } \\
\text { (\%) } \\
\end{array}$} & \multirow{2}{*}{$\begin{array}{l}\text { Grain } \\
\text { Density } \\
\text { (gm/ce) } \\
\end{array}$} & \multirow{2}{*}{$\begin{array}{c}\begin{array}{c}\text { Water } \\
\text { Saturation }\end{array} \\
(\%) \\
\end{array}$} & \multirow{2}{*}{$\begin{array}{c}\text { Oil } \\
\text { Saturation } \\
\text { (\$) }\end{array}$} & \multicolumn{2}{|c|}{ Fluorescense } \\
\hline & & Kmax & K90 & Kv & & & & & (\$) & Trpe \\
\hline & & & & & & & & & & \\
\hline 1 & 4024,08 & 45.8 & 5.1 & 5.7 & 4.1 & 2.69 & 19.2 & 0.0 & 0 & \\
\hline 3 & 4627.58 & 3.0 & 2.3 & 1.4 & 5.0 & 2.69 & 25.3 & 0.0 & 0 & \\
\hline 8 & 4630.68 & 204.0 & 164.0 & 62.2 & 13.3 & 2.68 & 17.0 & 0.0 & 0 & \\
\hline 7 & 4633.08 & 14.1 & 1.5 & 19.9 & 6.7 & 2.69 & 19.0 & 0.0 & 0 & \\
\hline 9 & 4636.68 & 108.0 & 90.4 & 0.9 & 6.2 & 2.69 & 21.7 & 0.0 & 0 & \\
\hline 11 & 4639.86 & 507.0 & 3120 & 123.0 & 5.4 & 2.70 & 13.6 & 0.0 & 0 & \\
\hline 13 & 4642,58 & 2.5 & 126.0 & 1.9 & 4.6 & 2.69 & 10.8 & 0.0 & 0 & \\
\hline 15 & 4645.68 & 46.1 & 28.2 & 7.6 & 6.8 & 2.69 & 0.6 & 0.0 & 0 & \\
\hline 17 & 4648.80 & 7.3 & 5.8 & 3.1 & 9.1 & 2.69 & 7.9 & 0.0 & 0 & \\
\hline 19 & 4651.68 & 1010.0 & 101.0 & 52.0 & 8.5 & 2.70 & 8.8 & 0.0 & 0 & \\
\hline 21 & 4654.68 & 111.0 & 82.0 & 80.8 & 12.2 & 2.70 & 8.9 & 0.0 & 0 & \\
\hline 23 & 4657.87 & 17,4 & 14.8 & 12.0 & 9.6 & 2.70 & 5.6 & 0.0 & 0 & \\
\hline 25 & 4660.68 & 14.2 & 7.3 & 5.0 & 6.2 & 2.69 & 9.7 & 0.0 & 0 & \\
\hline 27 & 4663.58 & 1460.0 & 1110.0 & 1150.0 & 8.3 & 2.69 & 12.0 & 0.0 & 0 & \\
\hline 29 & 466628 & 123.0 & 90.6 & 83.2 & 11,1 & 2.69 & 13.0 & 0.0 & 0 & \\
\hline 31 & 4669.68 & 36.5 & 5.7 & 61.9 & 9.8 & 2.69 & 9.0 & 0.0 & 0 & \\
\hline 33 & 4672.68 & 694.0 & 261.0 & 239.0 & 7.8 & 2.69 & 12.6 & 0.0 & 0 & \\
\hline 35 & 4675.68 & 480.0 & 386.0 & 129.0 & 10.4 & 2.70 & 10.3 & 0.0 & 0 & \\
\hline 37 & 4678.68 & 14.0 & 8.2 & 1.7 & 3.7 & 2.69 & 12.3 & 0.0 & 0 & \\
\hline 39 & 4681.68 & 320.0 & 120.0 & 1.4 & 5.4 & 2.70 & 7.4 & 0.0 & 0 & \\
\hline 41 & 4684.68 & 70.5 & 53.3 & 39.8 & 11.8 & 2.68 & 17.6 & 0.0 & 0 & \\
\hline 43 & 4687.38 & 277.0 & 80.4 & 118.0 & 8.7 & 2.65 & 18.2 & 0.0 & 0 & \\
\hline 45 & 4690.58 & 222000 & 0.0 & 8090.0 & 6.7 & 2.69 & 31.3 & 00 & 0 & \\
\hline 47 & 4693.58 & 5.5 & 4.3 & 1.2 & 5.9 & 2.69 & 9.6 & 0.0 & 0 & \\
\hline 49 & 4996.98 & 39.6 & 32.9 & 8.3 & 5.0 & 2.69 & 13.7 & 0.0 & 0 & \\
\hline 51 & 4699.68 & 89.9 & 16.8 & 1.1 & 7.1 & 2.69 & 10.1 & 0.0 & 0 & \\
\hline 53 & 4702.43 & 2.7 & 1.6 & 0.3 & 3.2 & 2.70 & 16.8 & 0.0 & 0 & \\
\hline 55 & 4705.68 & 719.0 & 178.0 & 10.4 & 4.7 & 2.70 & 14.0 & 0.0 & 0 & \\
\hline 57 & 4708.68 & 1450.0 & 656.0 & 61.3 & 4.8 & 2.60 & 13.6 & 0.0 & 0 & \\
\hline 59 & 4711.68 & 22.5 & 5.4 & 3.5 & 6.7 & 2.70 & 8.0 & 0.0 & 0 & \\
\hline 61 & 4714.68 & 866.0 & 114.0 & 214.0 & 7.2 & 2.70 & 11.4 & 0.0 & 0 & \\
\hline 63 & 4717.68 & 1700.0 & 1390.0 & 105.0 & 10.7 & 2.67 & 13.5 & 0.0 & 0 & \\
\hline 65 & 4720.88 & 88.6 & 70.8 & 57.3 & 13.8 & 2.69 & 9.0 & 0.0 & 0 & \\
\hline 67 & 4723.68 & 9.0 & 7.7 & 4.0 & 10.2 & 2.69 & 7.5 & 0.0 & 0 & \\
\hline 69 & 4726.68 & 7.3 & 2.5 & 3.6 & 7.1 & 2.69 & 9.2 & 0.0 & 0 & \\
\hline 71 & 4729.48 & 42.0 & 30.5 & 23.1 & 9.0 & 2.69 & 10.8 & 0.0 & 0 & \\
\hline 73 & 4732.73 & 250.0 & 134.0 & 548.0 & 0.0 & 2.69 & 122 & 0.0 & 0 & \\
\hline 75 & 4735.68 & 55.7 & 12.6 & 33.7 & 8.1 & 2.68 & 8.2 & tr & 50 & brt wh \\
\hline 77 & 4738.68 & 1.1 & 0.7 & 0.4 & 4.8 & 2.68 & 13.6 & tr & 50 & brt wh \\
\hline 79 & 4741,68 & 805.0 & 1720 & 810.0 & 12.1 & 2.69 & 11.5 & $t$ & 50 & brt wh \\
\hline 81 & 4744.68 & 325.0 & 1720 & 269.0 & 132 & 2.69 & 129 & tr & 50 & bit wh \\
\hline & & & & & & 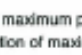 & & 100 & Ality & \\
\hline
\end{tabular}

Figure 4.13. Whole Core Analysis Onondaga Formation 


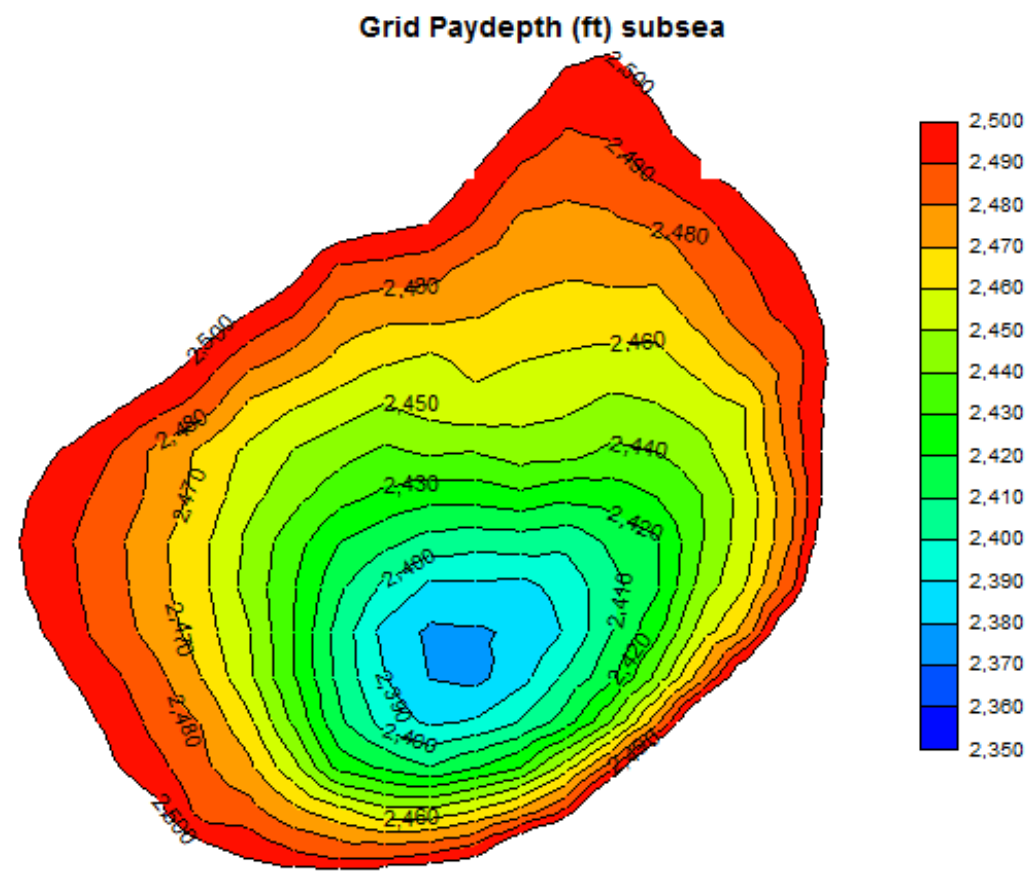

Figure 4.14. Structure Map Top of Onondoga Formation

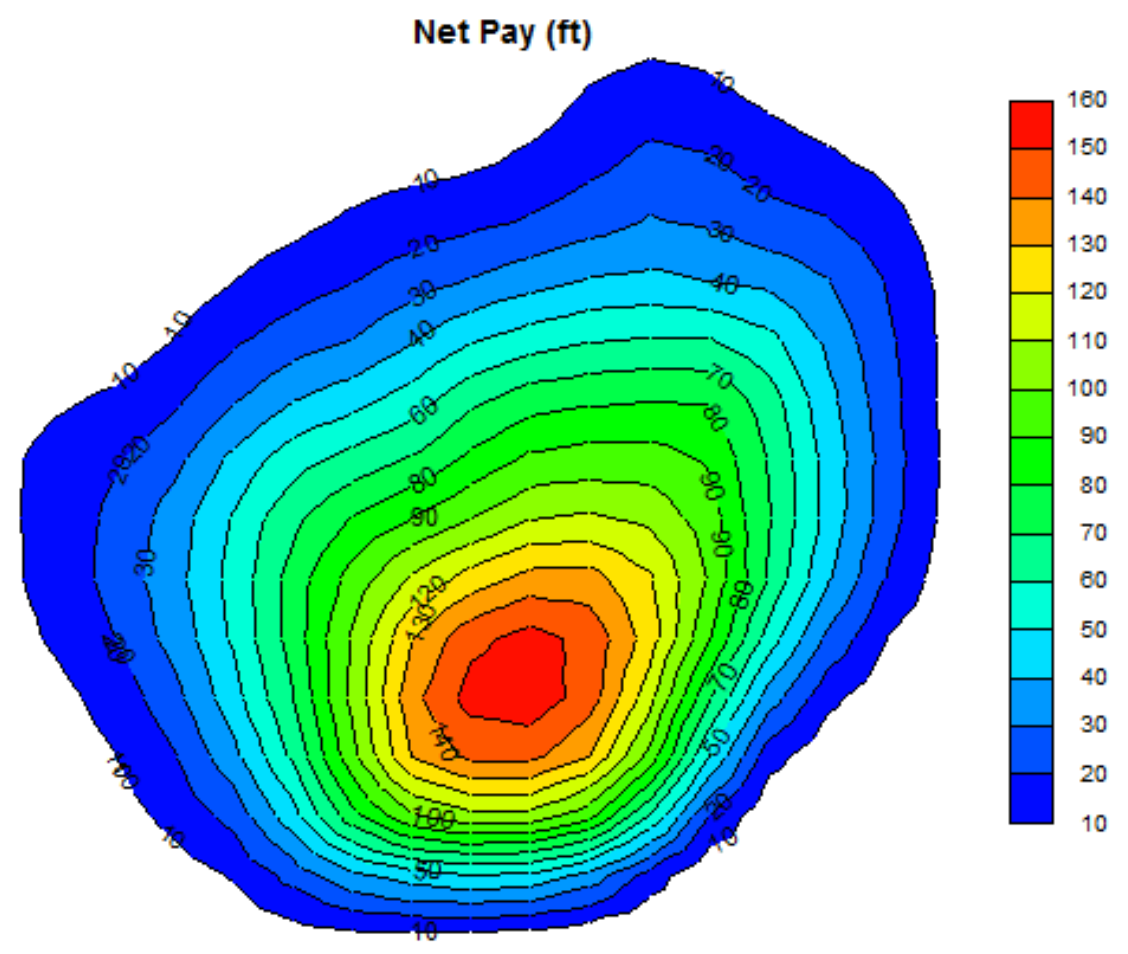

Figure 4.15. Net Isopach Map of Onondoga Formation 
Figure 4.14 shows the geologic interpretation of the Top of Onondaga Formation structure and Figure 4.15 shows the Net Isopach Map of Onondaga Formation based on the 3D seismic data obtained in 1999.

There have been Seven subsurface Onondaga reefs (Van Tyne 1995) found in southwestern New York (6) and northwestern Pennsylvania (1). The reef structures are very small less than 200 acres and a have maximum thicknesses of 200 feet. It has been reported that 30 smaller reefs in the same geologic section have previously been found along the Onondaga outcrop. The drilling of the QE-1 well in 1933 that penetrated the Quinlan reef is now thought to be the discovery well for the Onondaga reef gas. This well as mentioned above was located near Olean, NY in Cattaraugus County. The Wyckoff Reef was discovered in 1967 near Jasper, Steuben County, New York. See Figure 4.16 below for map showing geographic location of reefs. The Adrian Reef is of comparable size to the Quinlan reef approximately 8 BCF. Thomas Corners located near the town of Bath in Steuben County, New York is slightly larger at approximately 10 BCF.

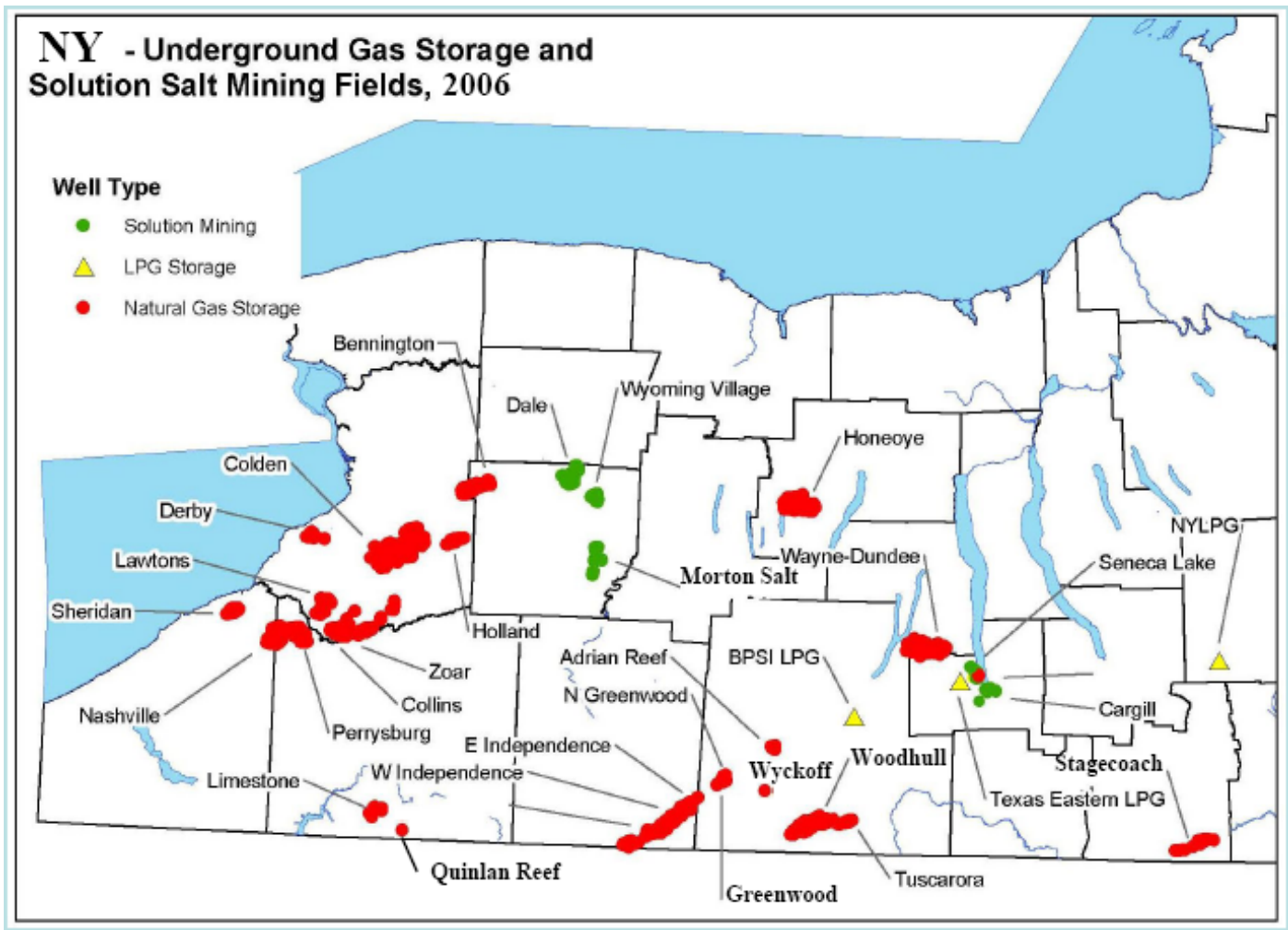

Figure 4.16. NY Geographic Map of Gas Storage (NYDEC Climate Change Workshop) 


\section{Open Hole Log Analysis}

The original well drilled in 1979 that produced the entire field never had a set of openhole logs ran due to problems encountered at the time of drilling. The observation well QW-3 was drilled in 1999 and was a vertical wellbore that allowed for openhole logs to be ran by Schlumberger for reservoir property analysis. The following well logs were ran:

\section{Schlumberger Platform Express Density/ Neutron/ Gamma Ray(Figure 4.17)}

2. High Resolution Azimuthal Laterlog(HALS)/Micro Gamma Ray(Figure 4.18)

3. Elemental Capture Spectroscopy Tool (ECS) (Figure 4.19)

4. Array Digital Sonic(Figure 4.20)

5. Combinable Magnetic Resonance Tool(CMR) (Figure 4.21)

6. ELAN (Elemental Analysis) PEX/ECS/HADL/CMR Log (Figure 4.22)

The GR/DEN/NEU log was run to detect fluids(hydrocarbons, water and gas) and porosity. The Laterolog was run to determine the water saturation. The digital sonic was run for rock properties determination. The CMR log was ran to determine the irreducible water fraction in the reservoir. The CMR and ECS log ran together were used to study the determination of the free and bound fluid. The Elemental Capture Spectroscopy (ECS) is for mainly lithology determination.

From Figure 4.17 the top of the Onondaga formation was picked from $4606 \mathrm{ft}$ and the bottom located at 4806 feet measured from ground level. This equated to a gross pay interval of 200 feet. Using the Density log and a matrix density of $2.71 \mathrm{~g} / \mathrm{cc}$ for limestone with a $5.9 \%$ porosity $(2.55 \mathrm{~g} / \mathrm{cc})$ cut off the net pay was calculated to be approximately 110 feet of net pay thickness. The log derived average porosity was $7.7 \%$ with a maximum porosity calculated to be $16.9 \%$.

The core analysis indicated a porosity ranging from $3.2 \%$ to $18.4 \%$ with an average of $9.1 \%$ from the cored section from 4608 to 4732 feet(124 ft). The reason the cored section porosity was higher than the log-derived porosity due to the core encompassed the better quality top part of the reef.

The Dual Induction Laterolog was used to arrive at an estimated water saturation(Sw) that could be used in developing an estimate to be incorporated into the reservoir model. 
The true formation resistivity for the Onondaga pay interval was estimated from the deep laterlog to average $1000 \mathrm{ohmm}$. When this was combined with the porosity and formation factor equated to a water saturation(Sw) of $4.9 \%(95 \%$ gas saturation(Sg)) for a limestone. The water resistivity used was $0.04 @ 103 \mathrm{~F}$. This seemed reasonable since the Quinlan production pool had no record of water production over the primary recovery time period.

The Array Digital Sonic was ran to develop a baseline mechanical properties of the formation competencies in case further studies needed to be done with breakdown pressures and drilling stresses.

The Elemental Capture Spectroscopy Tool (ECS) was run as a primary tool to determine the carbonate and quartz sections of the formation for better delineation of the reef. The tool allows for determination of the lithologic clay fractions.

The Combinable Magnetic Resonance Tool(CMR) high-resolution technology is important for formation evaluation, giving you free-fluid-index measurements. These measurements help to differentiate zones high and low potential water production zones.

Total CMR-200 log can estimate measurements of porosity by direct measurement of the echo spacings where the tiny pores can be evaluated. The CMR log confirmed the Free Fluid porosity of approximately $10 \%$ and that most capillary fluid was bound. The permeability was also confirmed from the CMR log to range from $1<k<100 \mathrm{md}$. The signal amplitude decay (relaxation) rate(T2) times were very high(> 300 millisecs) indicating that the small amount of fluid in the pore spaces had a relatively low viscosity. Most of the lower T2 bound fluid measurements were believed to be trapped condensate on the individual sand grains.

The ELAN(Elemental Analysis) Petrophysical log was used to correlate the Platform Express/CMR/ECS/HALS log data. The analysis confirmed the very low water saturation and permeability based on the pore size distribution from the T2 distribution. The relative permeability was calculated using the resistivity measurements to identify water zones. This analysis indicates that here is no producible water present and there is no appreciable liquid hydrocarbons(condensate) present that can be moved or produced. This is characteristic of high gas oil ratio retrograde condensate reservoirs. 
The processing also shows that fluid in the pore spaces is mostly irreducible water below $5 \%$ that compares favorably to the resistivity calculated values.

After QW3 was drill and completed there was Memory Production Log ran in 2000. This log was ran while the well was being flowed to the surface and indicated that a fluid gradient was present and gas cut fluid was present in open part of the wellbore.

A well test was completed on QW3 on November $18^{\text {th }}, 1999$ shortly after the well was completed. The purpose of the welltest was to determine the estimated reservoir $(k)$ permeability, the skin factor(s) and the deliverability constants(c and $\mathrm{n}$ exponent). The well was flowed to the atmosphere to establish the following parameters: permeability $(7.5 \mathrm{md})$, skin factor(4), n exponent(0.8), C( 0.053) and a AOF of $1.1 \mathrm{mmscfd}$

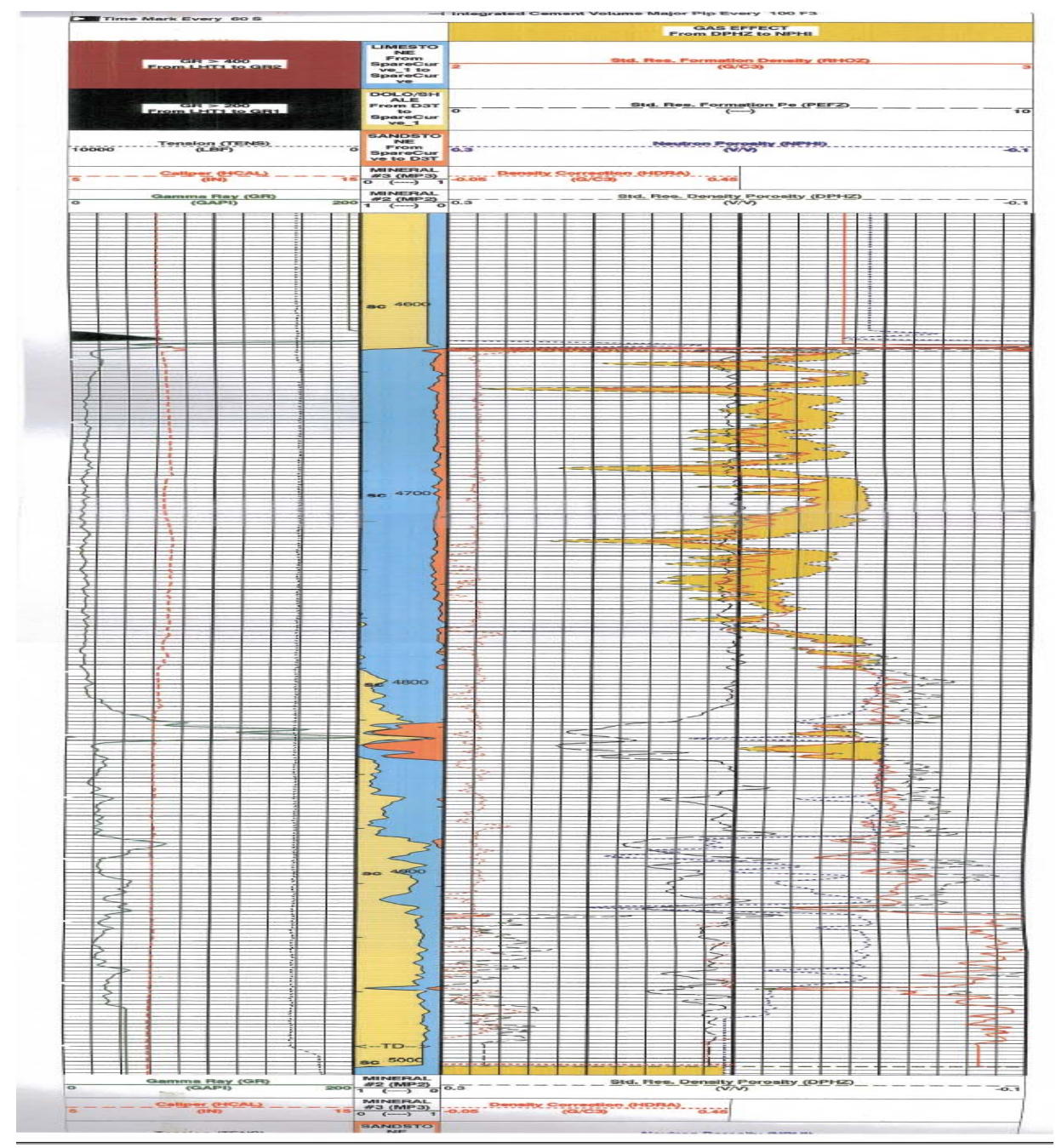

Figure 4.17. Platform Express Densityl Neutron/ Gamma Ray 


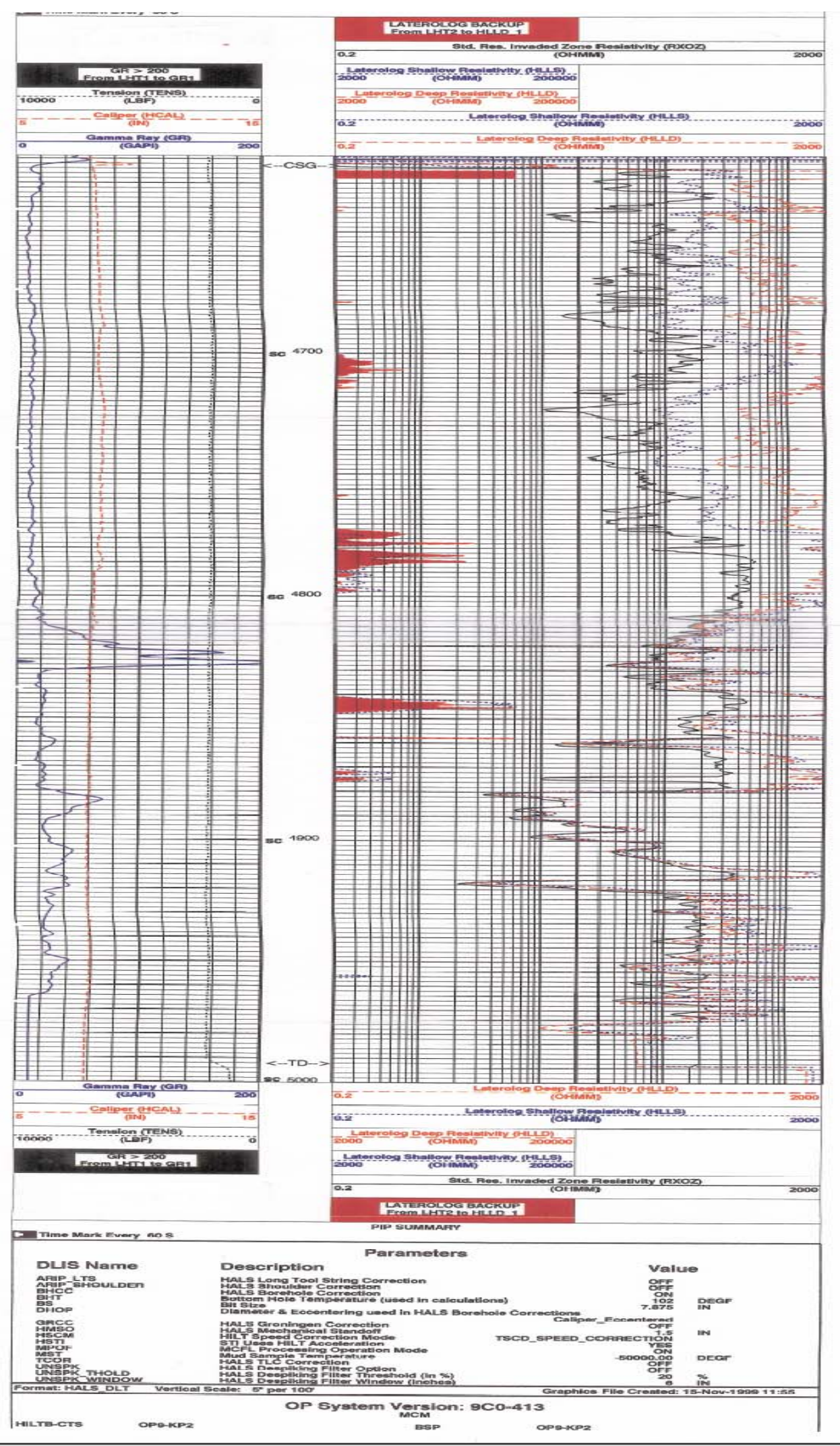

Figure 4.18. HALS Laterolog/Micro Gamma Ray 


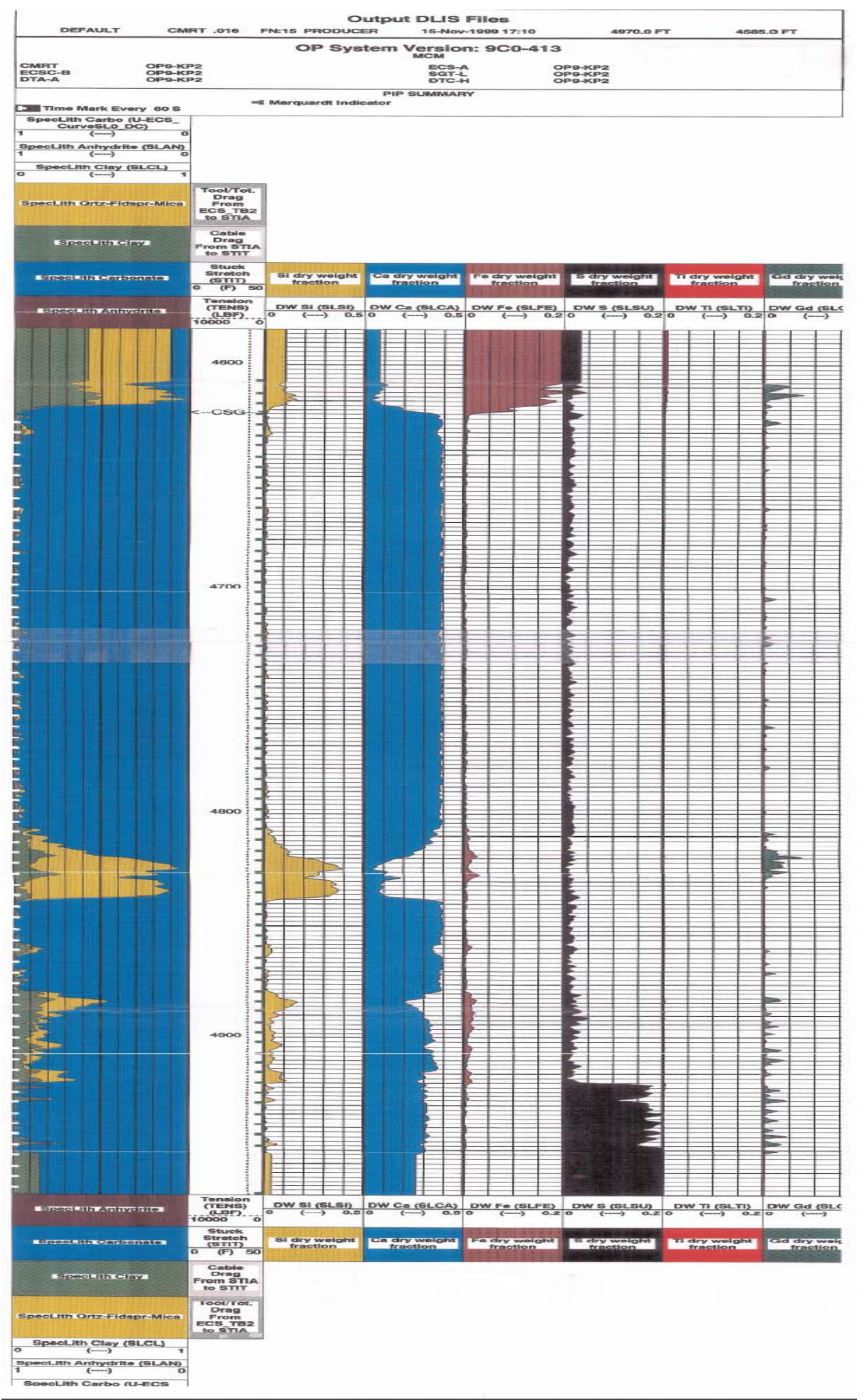

Figure 4.19. Elemental Capture Spectroscopy Tool (ECS) 


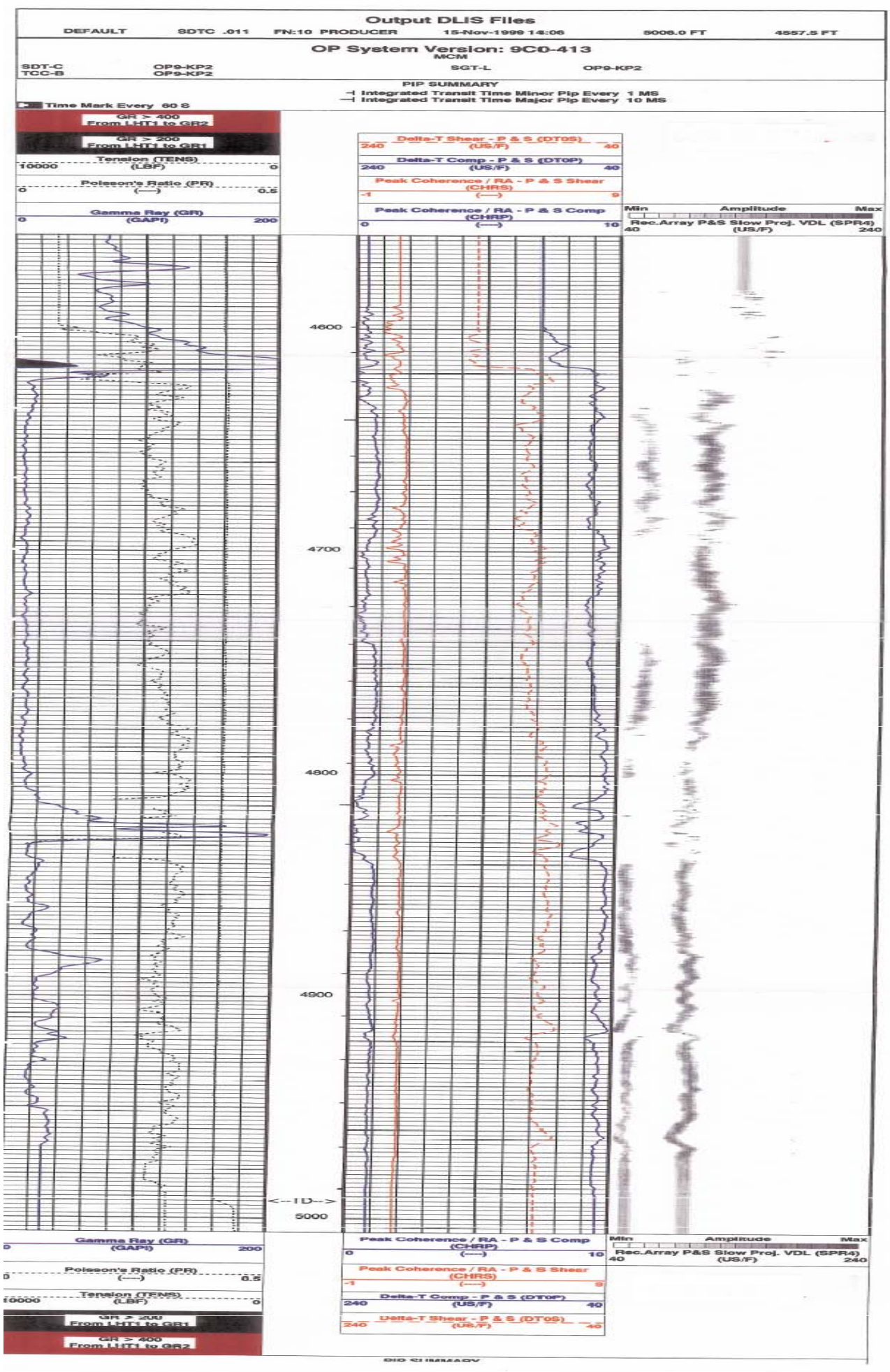

Figure 4.20. Array Digital Sonic 


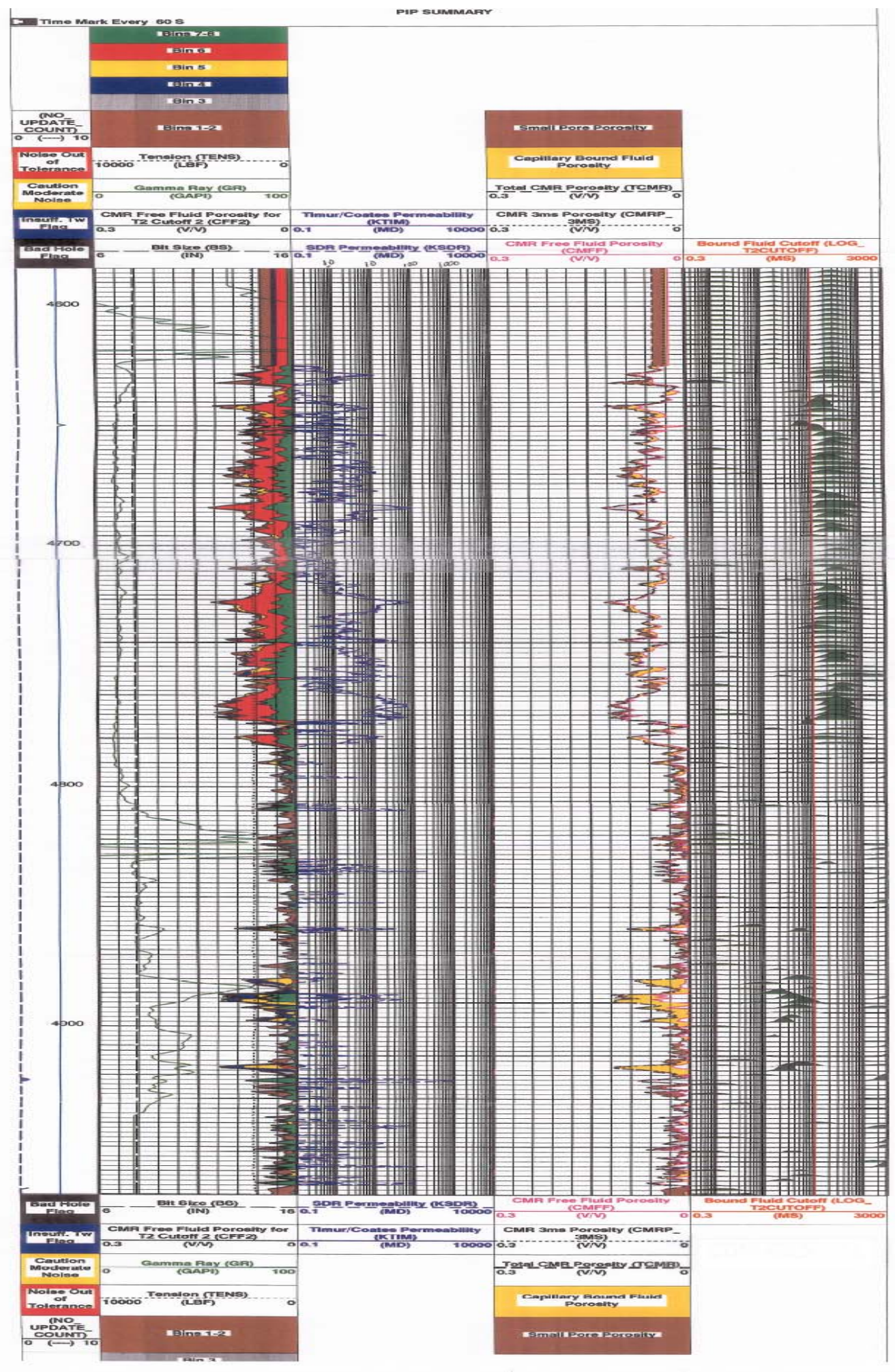

Figure 4.21. Combinable Magnetic Resonance Tool(CMR) 


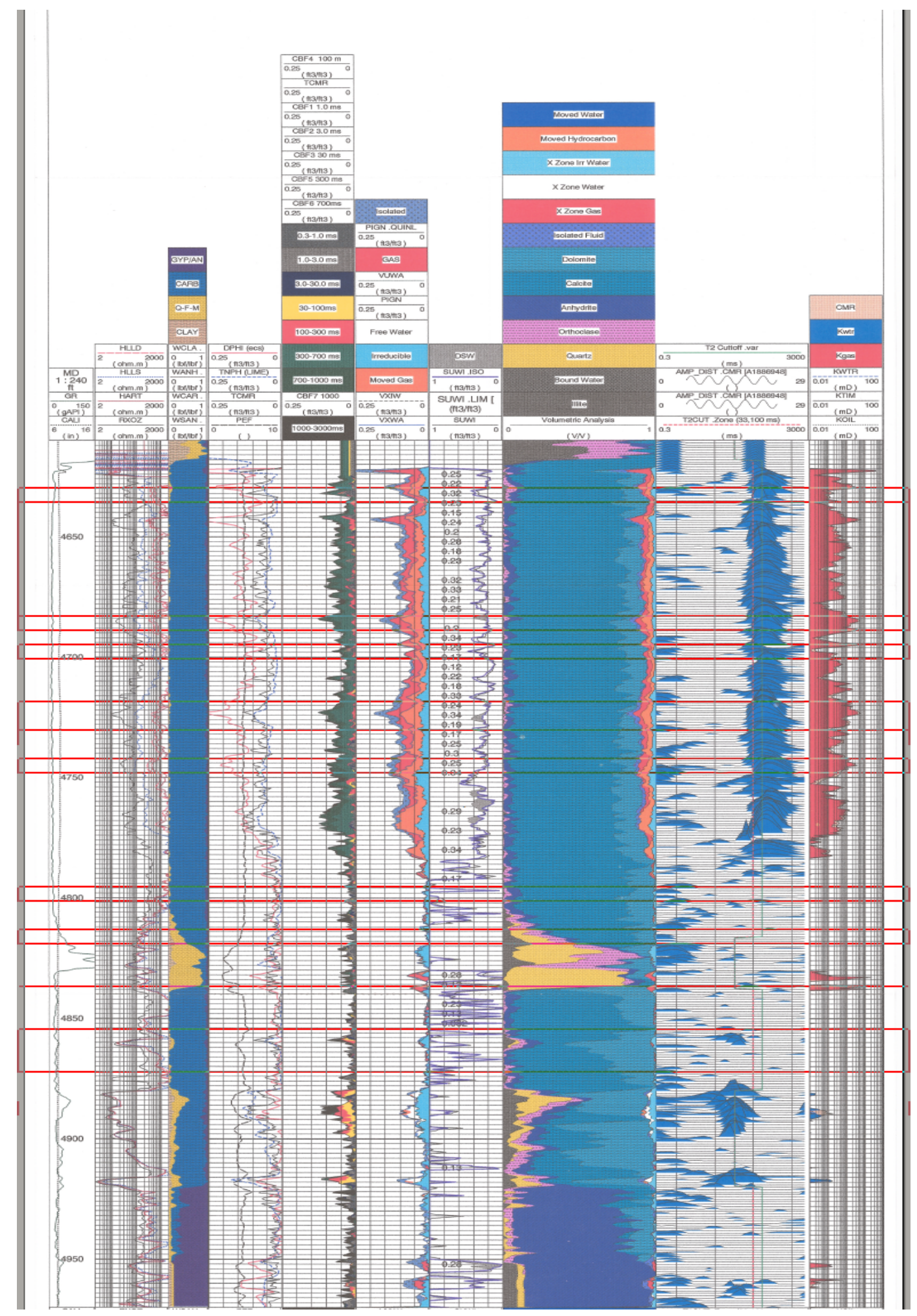

Figure 4.22. PEX/ECS/HADL/CMR ELAN Log 


\section{COMPOSITIONAL RESERVOIR MODELS}

Herzog (1980) described in an AGA paper "Retrograde Vaporization of Residual Condensation in Storage Field Development" that a natural gas condensate reservoir when depleted will contain some condensed natural gas liquids or condensate because of the retrograde condensation phenomenon. He also stated that the retrograde phenomenon can be theoretical reversed if the reservoir is repressurized and the condensate is revaporized. This revaporization process will result in the injected mix gas being enriched with the condensed hydrocarbons.

The introduction of phase behavior models has allowed the ability to predict gas quality and condensate recovery associated with storage operations. Katz(1981) authored a SPE paper on "Predicting Yield of Revaporized Condensate in Gas Storage". This paper addresses the use of phase behavior calculations for gas condensate systems and how they can be used to predict the degree of revaporization and resulting condensate yields from storage operations. Newer developments in simulation technology have enabled a compositional simulator to be utilized in conjunction with a full reservoir simulator. The effects of reservoir heterogeneities can then be studied to better understand the mixing and revaporization process.

In the Literature review Khamechi et al (2006) "Simulation of Underground Natural Gas Storage in Sarajeh Gas Field, Iran" used a coarse grid model to perform the history matching and storage simulations. The reservoir model in this Doctoral work was constructed using a fine grained reservoir simulation model coupled with the Peng Robinson Equation of State fluid model. The field was only partially depleted unlike the reservoir in this dissertation work that was mostly depleted.

The ability of the reservoir engineer to characterize the reservoir fluids and simulate compositional variations of those fluids from within the reservoir simulator allows for improved project design. The critical factors associated with the design and construction of the surface facilities and well placement and configuration can be accurately evaluated. The ability to accurately predict how these factors affect the mixing and recovery process improves the storage field development. 
Compositional analysis and pressure volume temperature relationship studies are rarely performed on newly discovered production reservoirs because they are cost prohibitive and difficult to obtain and analyze. This information is not critical to the production operation but is very important to studies relating to conversion to storage. Storage Operations can intensify the liquid recovery process because of the injected and withdrawn natural gas volumes can be much greater than volumes associated with production operations. The liquid yield estimates become very important in the storage field development phase because they can limit storage field performance and service.

Most PVT laboratory studies on gas condensate phase behavior are able to:

1. recombine separator liquid and gas into reservoir fluid

2. establish the retrograde dew point of the fluid

3. calculate well stream compositions

4. simulate well stream production

5. calculate cumulative stock tank liquid and gas recovery

6. estimate the volume of condensate condensed during depletion

These factors prove to be invaluable to the engineer working on the design of the facilities for conversion from production to storage. These studies particularly relating to the items listed above will have a strong affect on the conversion as we will discuss later.

Analysis of the gas and liquid hydrocarbon phases and composition is necessary to begin the storage conversion analysis. The phase behavior Equation of State(EOS) simulator is used to characterize the mixture. To start the analysis of the storage conversion analysis of the hydrocarbon mixture resident in the reservoir is necessary. 
The protocol for the use an Equation of State simulator would be:

1) estimate the extended compositional analysis of the reservoir fluid

2) vary the compositional analysis to match the laboratory phase behavior data

3) validate the estimated composition and molecular weight percentages with actual gas and liquid production.

4) estimate the remaining gas and liquid in place

5) use the calibrated EOS model with predictions for the storage phase

\section{RESERVOIR AND EOS MODEL SELECTION}

This study uses the Computer Modeling Groups GEM(General Equation of State Model)module that is a full compositional reservoir simulator. The phase behavior simulation was modeled using the CMG's WinProp based package for advanced modeling of the phase behavior and properties of reservoir fluids. A similar project was the study for conversion of the Sarajah Gas Field to gas storage that used the CMG GEM and Winprop simulation packages. (SPE 106341 2006)

The GEM(General Equation of State Model) is a robust compositional reservoir simulator that has the ability to process the mixing of solvent gas with native wet gas compositions and model the effects on fluid properties and yield prediction. The model has been used in the past for Coalbead Methane and Carbon dioxide(CO2) sequestration studies. The ability of GEM to model multiphase fluid flow in the any type of reservoir is strong point of the application. CMG states that GEM has been used any many production scenarios around the world where the interaction of fluids must be studied to evaluate the impact on the fluid recoveries. The application applied to storage scenarios where gas cycling and mixing must be evaluated is much less documented. The ability of GEM to provide for many different well management options is a plus because of the increased use of directional and horizontal well engineering solutions. The separator and gas plant staging modeling ability within GEM allows for the accurate representation of EOS modeling of the fluid from the reservoir to the surface facilities.

Gem also allows for relatively complex structures to be imported directly from geosciences and geologic interpretation applications. Important reservoir parameters can be manipulated for 
evaluation after they are imported. In summary the ability of GEM to model gas condensate and gas cycling was the reason it was selected for meeting our modeling requirements.

This study uses the Computer Modeling Groups WinProp is CMG's Windows ${ }^{\mathrm{TM}}$ based package for modeling the phase behavior and properties of reservoir fluids. WinProp is a comprehensive equation of state model that can be used in CMG's reservoir simulators. The WinProp interface is very user friendly and allows for exporting results for comparison of differing cases. When a gas condensate reservoir initial fluid characterization study is being performed the ease of running modeled laboratory experiments is necessary. WinProp allows for surface conditions as well as reservoir conditions to be modeled for compositional variations where phase equilibrium is important. The PVT matching features allow for easy comparison of modeled scenarios to actual laboratory data. The ability of WinProp to model multiple surface separation (EOS stages) was found to be a very valuable feature in this study. CMG's WinProp EOS application is an integral component for advanced reservoir simulation modeling.

\section{RESERVOIR MODEL DEVELOPMENT}

Understanding that the compositional reservoir model will require both an Equation of State model for the fluid characterization as well as the reservoir model for characterization of the reservoir properties, the reservoir model development procedure was divided into the following two (2) primary sections:

1) Fluid Model characterization(PR-EOS and WinProp EOS)

a. PVT Laboratory versus EOS model comparisons

b. Simulation of Primary production

c. Material Balance Studies

2) Compositional Model Development
a. Reservoir model characterization
b. Integration of WinProp Phase Behavior Model
c. History Matching of Primary production
d. Simulation of Storage Scenarios 


\section{FLUID MODEL CHARACTERIZATION}

\section{Initial Reservoir Fluid Evaluation(PR-EOS)}

The Peng Robinson (PR) Equation of State phase behavior package by Aminian (1989) was used for the initial fluid analysis. This package was used to simulate retrograde condensation in the reservoir caused by the primary production. After a suitable match was achieved for the fluid properties the EOS was used to simulate storage injections and withdrawals and to predict the condensate gas ratios or liquid yields on storage withdrawals.

Typically on condensate gas reservoirs laboratory analysis can be found on the reservoir fluid collected from the separator. This fluid is usually in the liquid and vapor phases from this the following parameters can be determined:

1. The producing gas- liquid ratio

2. Independent compositional analysis on the gas and liquid.

3. Surface recoverable products converted to well stream volumes

The separator gas and liquid is then recombined and then analyzed as a reservoir fluid at reservoir condition of temperatures and pressure:

1) Pressure volume relationship

2) Reservoir fluid composition at original reservoir conditions.

An important phase in the development is to accurately understand the initial and final fluid properties by characterizing then with an equation of state model. This proves to be very difficult due to the fact initial fluid property studies are rarely needed when production begins and if they are available are rarely in the detail needed to incorporate into a reservoir fluid compositional simulator. Therefore when construction of the fluid compositional simulator is under development care must be taken to use the proper analysis methods so not to arrive at fluid properties that may later prove to be flawed. 
At the beginning when the storage conversion process was being studied to determine if the project is feasible, research began on data that supported fluid compositional analysis. After the first discovery well and later the sole production well for the entire pool was drilled a PVT analysis was performed that extensively studied the reservoir fluid properties. In August 1979 a PVT laboratory study was performed using liquid and vapor collected from the separator. Tables 4.1, 4.2 and 4.3 summarize the data contained in the original PVT report. The reservoir pressure is thought to have been at the dew point of the original lean condensate reservoir fluid where the gas and liquid were in equilibrium.

\begin{tabular}{|l|c|}
\hline Sampling Conditions & \\
\hline & \\
\hline Primary Tubing Pressure & $1980 \mathrm{psig}$ \\
\hline Primary Separator Pressure & $515 \mathrm{psig}$ \\
\hline Primary Separator Temp & $54 \mathrm{~F}$ \\
\hline Reservoir Temp & $120 \mathrm{~F}$ \\
\hline Reservoir Pressure & $2710 \mathrm{psig} @ 4333 \mathrm{ft}$ \\
\hline Field Stock Liquid Gravity @ 60 F & $64.2 \mathrm{degrees} \mathrm{API}$ \\
\hline Separator Gas Prod Rate & $2500 \mathrm{mscfd}$ \\
\hline Pressure Base & 14.73 \\
\hline Temperature Base & 60 \\
\hline Compressibiltiy Factor & 0.715 \\
\hline Gas Gravity (Lab) & 0.73 \\
\hline Condensate Liq Rate @ 60 F & $120 \mathrm{bbls} / \mathrm{day}$ \\
\hline Primary Separator Gas /Condensate Liq Ratio & $20.833 \mathrm{mscf} / \mathrm{bbl}$ \\
\hline Condensate Gas Ratio & $48 \mathrm{bbl} / \mathrm{mm}$ \\
\hline
\end{tabular}

Table 4.1. Sampling Conditions(1979 PVT data)

Constant Volume Depletion Results $\quad$ Mol \%

\begin{tabular}{|c|c|c|c|c|c|c|c|c|}
\hline \multicolumn{3}{|c|}{ Reservoir Pressure(psia) } & 2700 & 2100 & 1500 & 900 & 500 & 0 \\
\hline \multicolumn{2}{|c|}{ Carbon Dioxide } & & --- & --- & --- & --- & --- & --- \\
\hline Nitrogen & & & 0.41 & 0.40 & 0.41 & 0.41 & 0.42 & 0.42 \\
\hline $\mathrm{CH} 4$ & Methane & & 73.22 & 74.48 & 75.19 & 75.51 & 75.02 & 72.75 \\
\hline $\mathrm{C} 2 \mathrm{H} 6$ & Ethane & & 13.85 & 13.90 & 13.94 & 13.98 & 13.88 & 13.59 \\
\hline $\mathrm{C} 3 \mathrm{H} 8$ & Propane & & 5.78 & 5.68 & 5.61 & 5.60 & 5.87 & 6.57 \\
\hline iC4H10 & i-Butane & & 0.85 & 0.79 & 0.76 & 0.74 & 0.88 & 1.17 \\
\hline $\mathrm{C} 4 \mathrm{H} 10$ & Butane & & 1.96 & 1.86 & 1.78 & 1.76 & 1.98 & 2.52 \\
\hline iC5H12 & i-Pentane & & 0.53 & 0.44 & 0.41 & 0.40 & 0.42 & 0.62 \\
\hline $\mathrm{C} 5 \mathrm{H} 12$ & Pentane & & 0.71 & 0.60 & 0.55 & 0.52 & 0.54 & 0.83 \\
\hline $\mathrm{C} 6 \mathrm{H} 14$ & Hexane & & 0.95 & 0.67 & 0.55 & 0.44 & 0.45 & 0.72 \\
\hline \multirow[t]{2}{*}{$\mathrm{C} 7+$} & Heptanes & & 1.74 & 1.18 & 0.80 & 0.64 & 0.54 & 0.81 \\
\hline & & & 100.00 & 100.00 & 100.00 & 100.00 & 100.00 & 100.00 \\
\hline \multicolumn{3}{|c|}{ Molecular Wt of Heptanes plus } & 131 & 112 & 106 & 104 & 106 & 112 \\
\hline \multicolumn{3}{|c|}{ Specific Gravity of Heptanes plus } & 0.742 & 0.720 & 0.717 & 0.712 & 0.718 & 0.726 \\
\hline \multicolumn{9}{|c|}{ Deviation factor - Z } \\
\hline \multicolumn{2}{|c|}{ Equilibrium gas } & & 0.759 & 0.736 & 0.753 & 0.806 & 0.872 & --- \\
\hline \multicolumn{2}{|c|}{ Two-phase } & & 0.759 & 0.739 & 0.75 & 0.798 & 0.858 & --- \\
\hline \multicolumn{9}{|c|}{\begin{tabular}{|l|} 
Wellstream produced- \\
\end{tabular}} \\
\hline \multicolumn{3}{|c|}{ cumulative $\%$ of initial } & 0 & 22.512 & 47.718 & 71.315 & 86.646 & 99.085 \\
\hline
\end{tabular}

Table 4.2. Constant Volume Depletion PVT Results(1979 PVT data) 


\section{Constant Composition Expansion Results}

\begin{tabular}{|l|l|}
\hline Dew Point & $2700 \mathrm{psig}$ \\
z factor & 0.759 \\
Gas Formation Volume Factor & $1.229 \mathrm{mscf} / \mathrm{bbl}$ \\
\hline
\end{tabular}

\section{Table 4.3. Constant Composition Expansion(1979 PVT data)}

The storage facilities must be designed to be able to process these revaporized liquids that are ultimately produced from the storage reservoir much like a secondary recovery operation.

Aminian et al (SPE 91483 2004) developed a methodology to study the influences that would affect the successful development of storage operations. The methodology in order used to study the influences was:

1) Comparison with Laboratory PVT Study

2) Simulation of Primary Condensate Production

3) Material Balance Study

4) Simulation of Storage

PVT(Pressure, Volume and Temperature) studies are used to analyze the reservoir fluid properties. Typically, these studies are ran at reservoir and surface separation facility conditions. These PVT studies when conducted on gas condensate reservoir fluids typically consist of the following experiments or tests:

Constant Composition Expansion(CCE) and Constant Volume Depletion(CVD) tests. The CVD tests are performed on reservoir fluids such as gas condensate mixtures to simulate the conditions or reservoir depletion. The sample is held at the saturation dew point pressure in a closed cell and then the pressure is reduced by increasing the cell volume. Gas is then liberated from the mixture and then the gas is bled off until the mixture is back at the original cell volume. This process is repeated for several pressure increments. The CCE is also referred to as flash liberation or vaporization. The pressure in the cell is reduced by increasing the cell volume and the fluid gas and liquid phases are measured. Other properties such as viscosities, compressibility factors and densities can also be measured. 
An extended compositional analysis of the original reservoir fluid was not available. Ahmed (1985) stated that Equation of State models can not accurately predict phase behavior without detailed analysis of the heptanes plus fractions. He proposed a method of splitting the plus fractions into psuedocomponents.

Several different $\mathrm{C} 6+$ splitting cases were developed and used in the PR-EOS phase behavior package to compare to the extended compositional laboratory PVT studies. The laboratory measurements provided dew point pressure, cumulative gas produced, percentage liquid dropout, gas deviation factor and compositional data at predetermined pressure increments.

To evaluate the initial PVT data and results the recombined separator sample analysis was evaluated with an Equation of State model results. Careful evaluation was made considering of the know problems with this type of sampling and analysis. Whitson has said in the Literature review that EOS models have been shown to not be very effective in matching the retrograde phenomena specifically gas compostional behavior and liquid formation of the system near or just below the initial dewpoint.

The two methods (model versus laboratory results) of initial fluid composition analyses had to be compared before any further steps could be taken with the methodology. The following fluid property indicators were used in the comparison: cumulative gas production, produced gas gravity, molecular weight of $\mathrm{C} 7+$ in the produced gas, 2-phase z-factors, and the volume percent of condensate in the reservoir at various reservoir pressures. A PR-EOS phase behavior package(Aminian 1989) was utilized to simulate retrograde condensation in the reservoir and to predict the liquid yield during storage withdrawal cycles.

After some degree of confidence could be given to the initial fluid composition characterization careful progress was made towards using the EOS model to compare the primary production of natural gas and condensate liquids recovered. 
A regression study was performed using the Peng -Robinson EOS phase behavior package developed by Aminian(1989). There were forty(40) cases ran using different composition arrays to compare to the laboratory parameters described above. As a result of this study the Case 33 composition that is shown below in Table $\mathbf{4 . 4}$ exhibited the best match for: dew point pressure, cumulative gas produced, percentage liquid dropout, gas deviation factor and compositional data at predetermined pressure increments. The retrograde dewpoint pressure was calculated to be 2714.7 psia.

\begin{tabular}{|l|r|}
\hline \multicolumn{1}{|c|}{ Component } & Case 33 \\
\hline $\mathrm{N} 2$ & 0.41 \\
\hline METH & 73.22 \\
\hline ETHN & 13.85 \\
\hline PROP & 5.78 \\
\hline $1-\mathrm{C} 4$ & 0.85 \\
\hline $\mathrm{N}-\mathrm{C} 4$ & 1.96 \\
\hline $\mathrm{I}-\mathrm{C} 5$ & 0.53 \\
\hline $\mathrm{N}-\mathrm{C} 5$ & 0.71 \\
\hline $\mathrm{C} 6$ & 0.95 \\
\hline $\mathrm{C} 7$ & 0.57440 \\
\hline $\mathrm{C} 8$ & 0.54340 \\
\hline $\mathrm{C} 9$ & 0.22720 \\
\hline $\mathrm{C} 10$ & 0.12770 \\
\hline $\mathrm{C} 11$ & 0.08120 \\
\hline $\mathrm{C} 12$ & 0.05030 \\
\hline $\mathrm{C} 13$ & 0.03000 \\
\hline $\mathrm{C} 14$ & 0.02500 \\
\hline $\mathrm{C} 15$ & 0.01900 \\
\hline $\mathrm{C} 16$ & 0.01320 \\
\hline $\mathrm{C} 17$ & 0.01200 \\
\hline $\mathrm{C} 18$ & 0.00960 \\
\hline $\mathrm{C} 19$ & 0.00780 \\
\hline $\mathrm{C} 20$ & 0.00680 \\
\hline $\mathrm{C} 21$ & 0.00600 \\
\hline $\mathrm{C} 22$ & 0.00730 \\
\hline & 100.0 \\
\hline
\end{tabular}

Table 4.4. PR-EOS Case 33 composition breakdown

\section{Comparison with Laboratory PVT Study}

To evaluate the accuracy of the extended compositional analysis, the predicted retrograde dewpoint pressure was compared against the laboratory measured value. The initial extended compositional analysis did not generate a close match. Therefore, extended compositional analysis was modified to obtain a close match with laboratory measured retrograde dewpoint pressure. Upon obtaining a close match with dew point pressure, a number of indicators were utilized for comparison against laboratory results. They included cumulative gas production, produced gas gravity, molecular weight of $\mathrm{C} 7+$ in the produced gas, 2-phase $z$-factors, and the 
volume percent of condensate in the reservoir at various reservoir pressures. Figures $4.23 a$, 4.23b, 4.23c, 4.23d and 4.23e illustrates the various comparisons. The results of simulation studies do not completely agree with the laboratory results but they were within reasonable ranges. The key indicator for purposes of storage performance simulations is the volume percent of condensate in the reservoir. It is important to note that even though the predicted liquid volume percents are higher than those measured in the laboratory study, the volume percent of liquid in the reservoir at time of conversion to storage is relatively close to the laboratory measured value. This is because the simulated results indicate more liquid evaporation at lower pressures (below 500 psig).

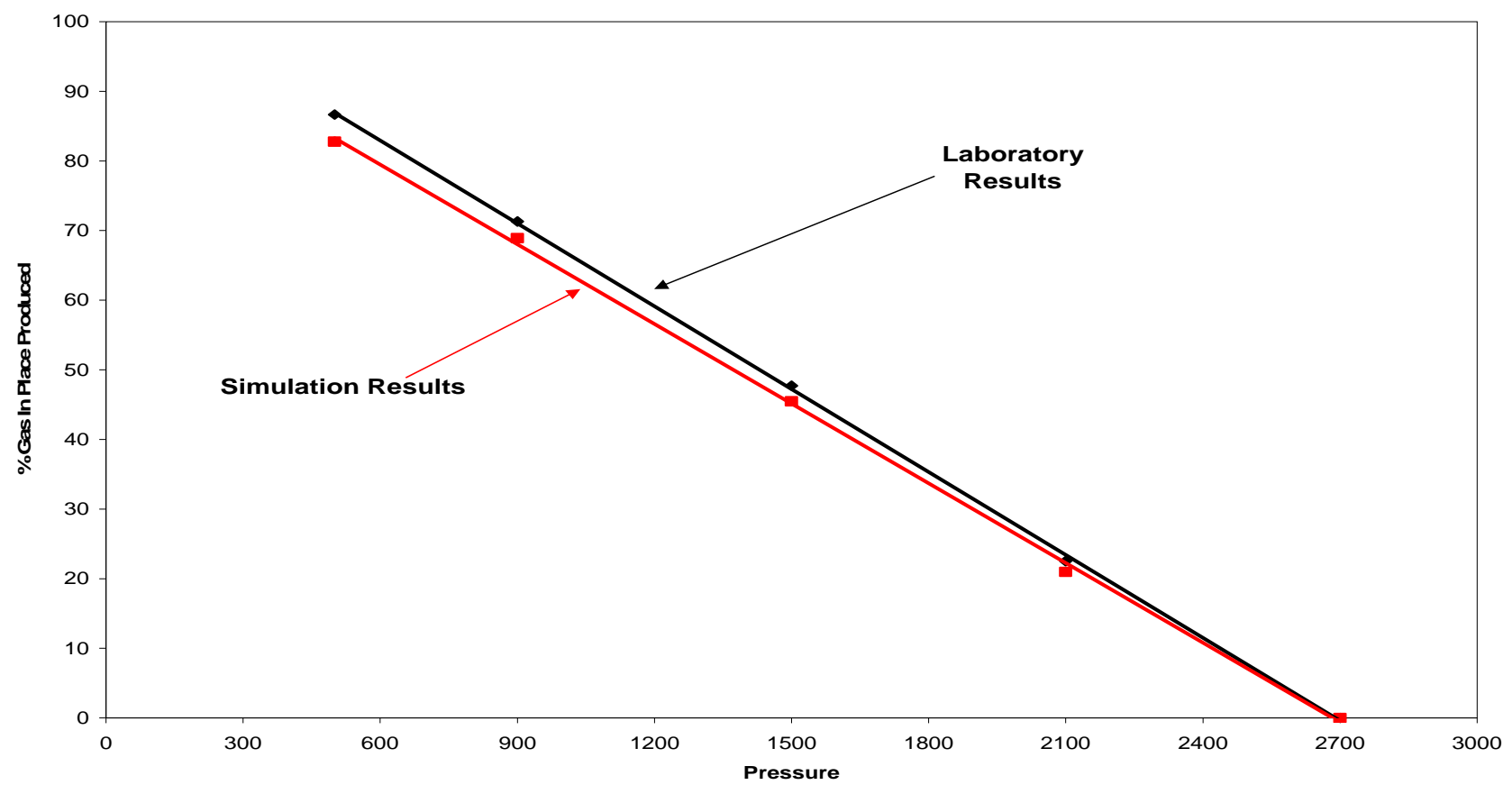

Figure 4.23a: \% Gas In Place Produced 


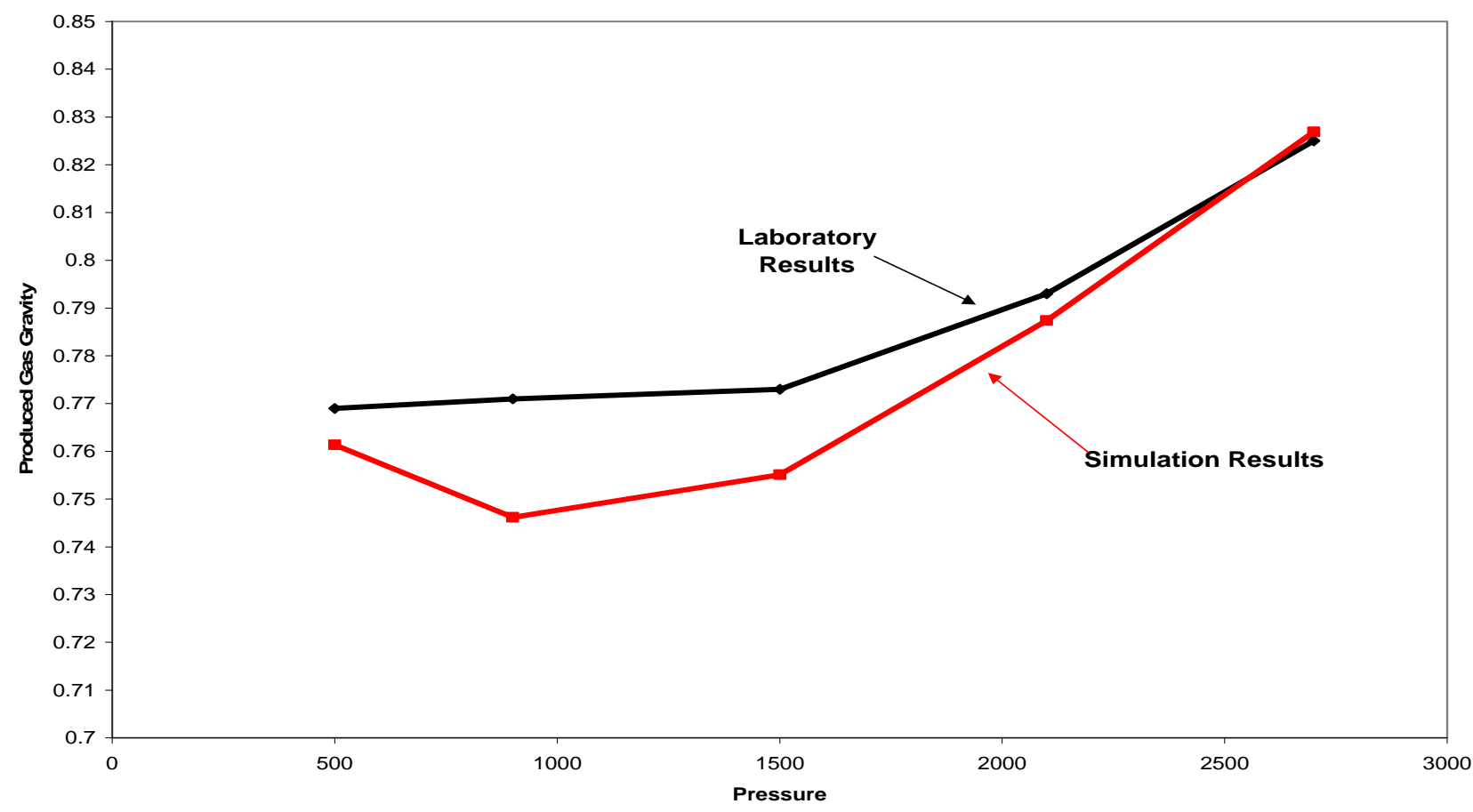

Figure 4.23b: Produced Gas Gravity

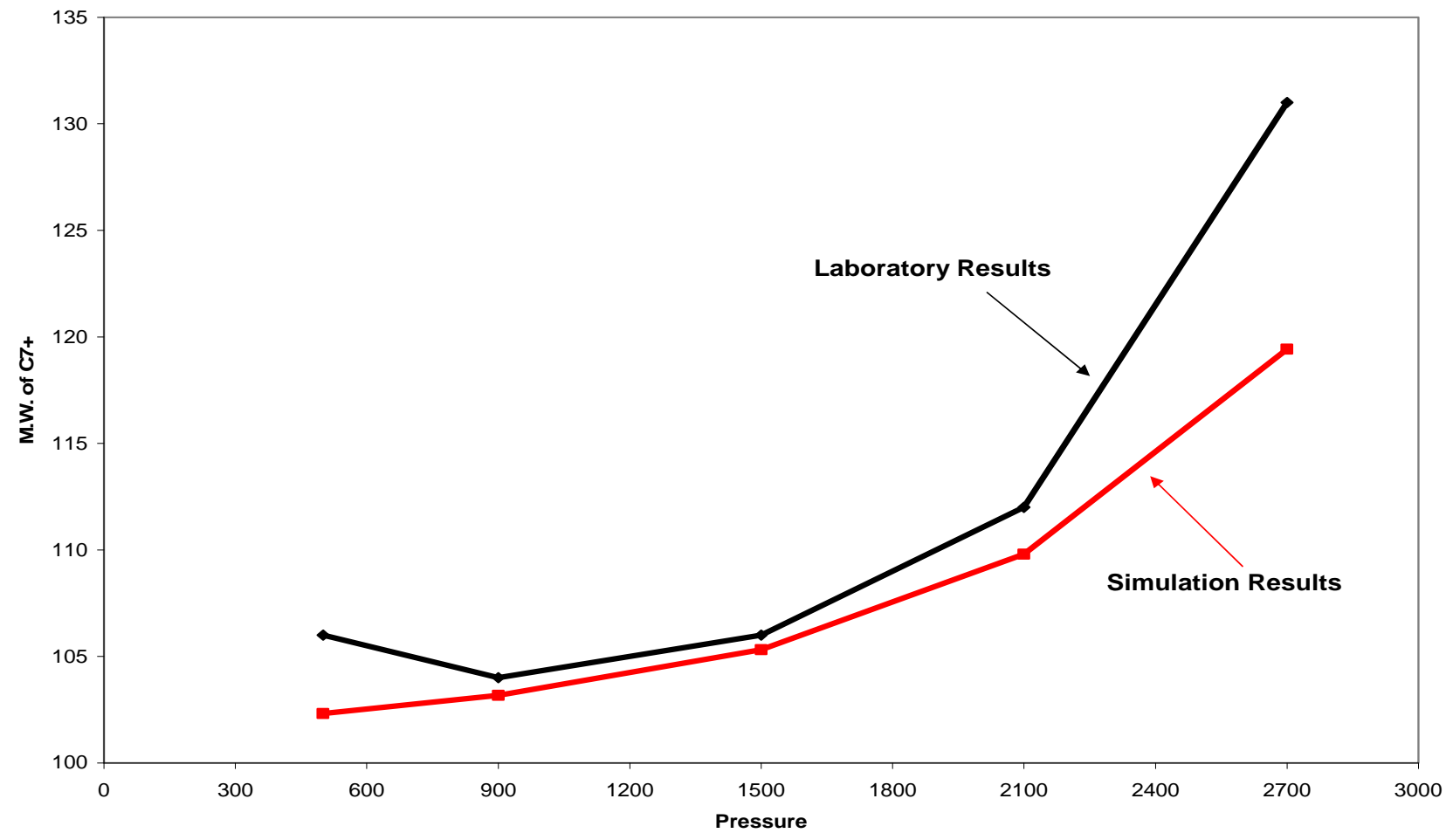

Figure 4.23c: M.W. of C7+ 


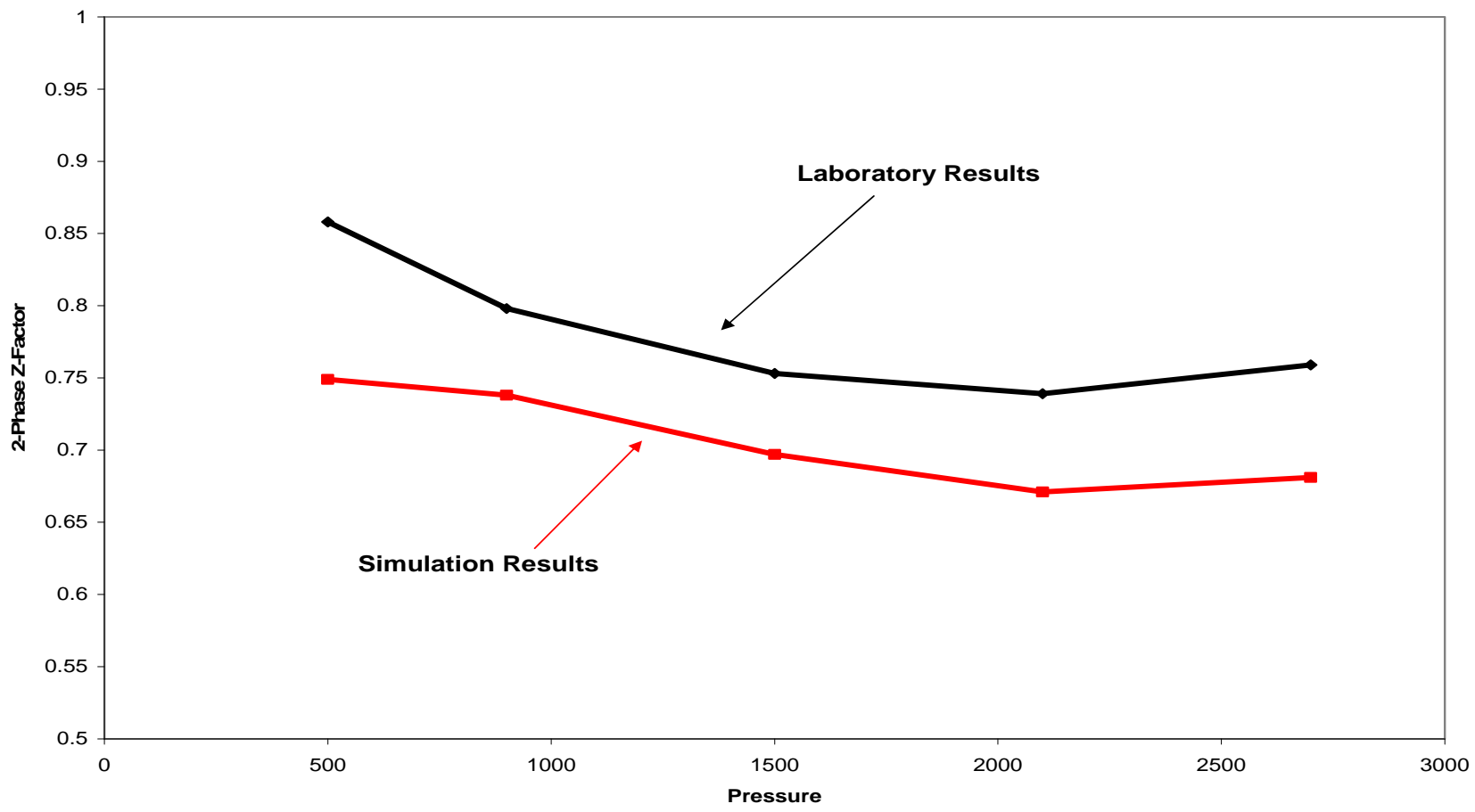

Figure 4.23d: 2- Phase $z$ factor

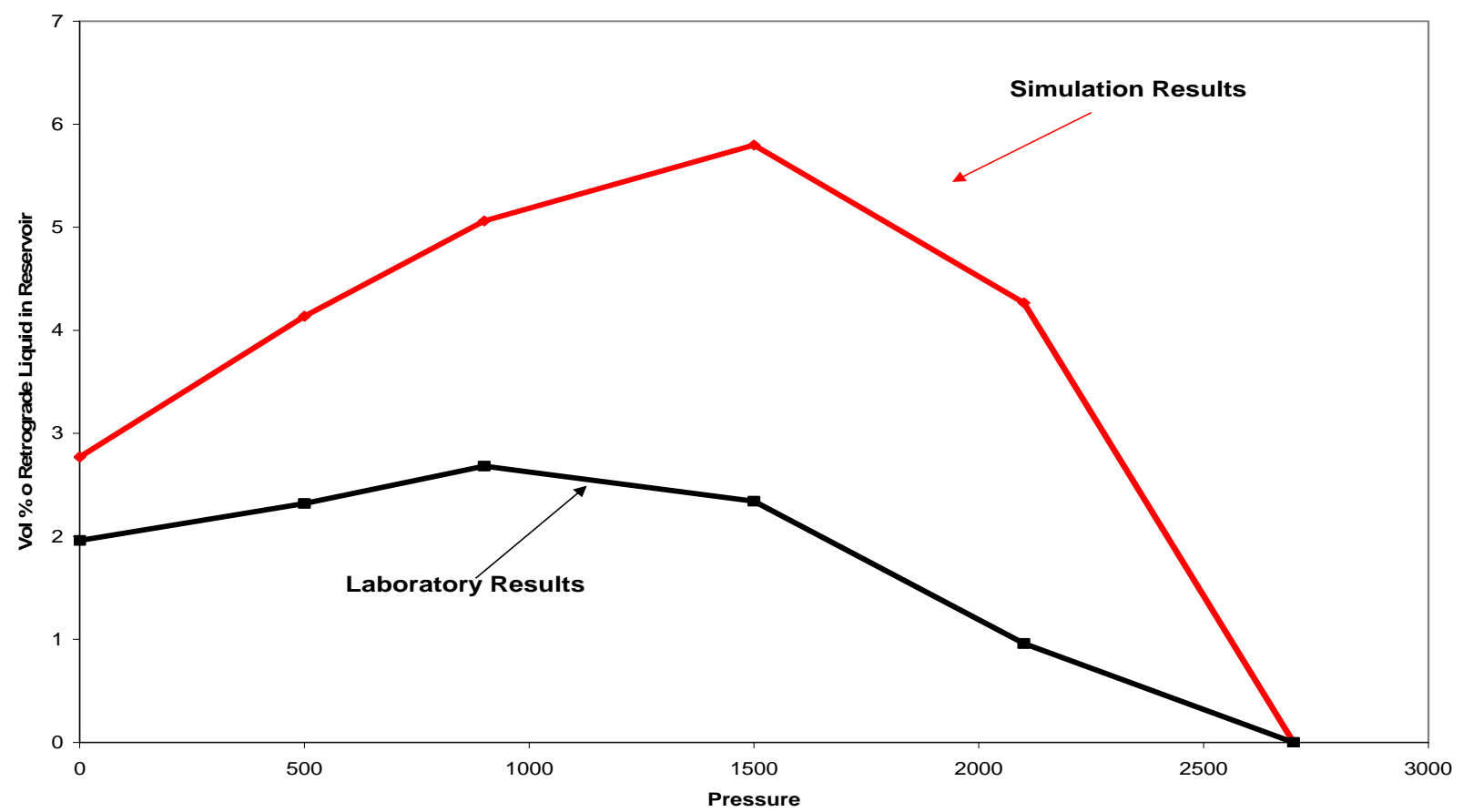

Figure 4.23e: \% Retrograde Liquid in Reservoir 


\section{Comparison with Primary Liquids Production History}

Figure 4.24 illustrates the comparison of predicted and field data on average liquid yields. Again, they predicted values are within reasonable ranges. It should be noted that accurate separator conditions were not available and they were estimated. These assumptions obviously influence the results of the predictions. The similarity between the predicted and measured field liquid yields further confirms that PR-EOS phase behavior package can provide reliable results for predicting retrograde condensation in the reservoir.

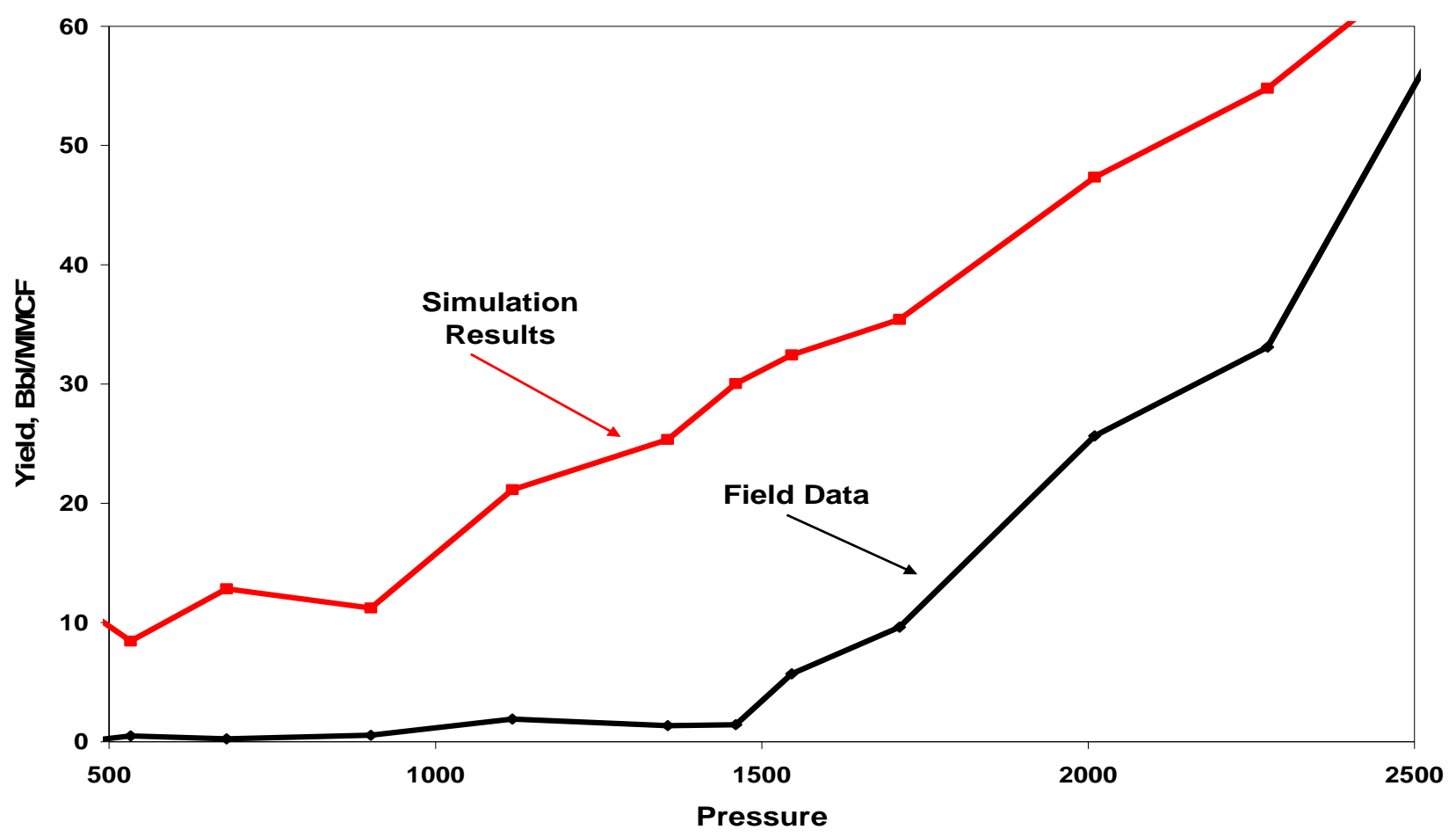

Figure 4.24: Comparison of EOS-Predicted and Field Liquid Yields

The flowing gas containing retrograde liquid mixes with existing liquids when the pressure declines and the flowing oil has solution gas dissolved that when the pressure is reduced vaporizes and mixes with the existing gas phase. This phenomenon cannot be simulated in the laboratory or with complex component phase behavior models. The Peng- Robinson EOS calculated liquid densities from the simulated CVD (Constant Volume Depletion) data were always lower than the laboratory measured values. This is one of the reasons Whitson et al(1983) concluded that the EOS programs overestimate liquid volumes from reservoir fluids. 


\section{Initial Reservoir Fluid Evaluation(WinProp)}

In order to use the compositional reservoir simulator, GEM, it is necessary to first generate the phase behavior prediction model for the reservoir fluid. WinProp is an EOS-based phase behavior simulator that is incorporated in GEM for this purpose. WinProp can split the heavy end (C7+ fraction) into a number of pseudo components and has a flexible component lumping option. WinProp also has a robust adaptive regression algorithm for tuning the EOS parameters to match laboratory PVT data. The estimated extended original fluid compositional analysis and the results of the laboratory PVT study were used as inputs to WinProp.

The predicted phase behavior utilizing component lumping and the regression analysis provided similar results as those previously obtained by PR-EOS phase behavior simulation package. This confirmed the reliability of the estimated extended compositional analysis and also provided the necessary phase behavior input for GEM compositional model.

A regression study was done using the CMG WinProp phase behavior package and the final composition described in Case 33 shown in Table 4.4. Saturation Pressure and equilibrium properties calculations were at 2716.483 psia and 120 deg F. Tables 4.5,4.6 4.7, 4.8 and Figure 4.25 summarize the WinProp output. 
Constant volume depletion calculation

Results after regression

Summary of Constant Volume Depletion at $120 \mathrm{deg} F$

Hydrocarbon Analyses of Produced Gas Phase - Mole \%

\begin{tabular}{|c|c|c|c|c|c|c|c|}
\hline \multirow{2}{*}{\begin{tabular}{|l|} 
Component \\
\end{tabular}} & \multirow[b]{2}{*}{2716.5} & \multirow[b]{2}{*}{2114.7} & \multicolumn{5}{|c|}{ Composition (mole \%) at Pressure Levels (psia) } \\
\hline & & & 1514.7 & 914.7 & 514.7 & 14.7 & 14.7 \\
\hline N2 & 0.41 & 0.42 & 0.43 & 0.43 & 0.41 & 0.35 & 0 \\
\hline C1 & 73.22 & 74.59 & 75.96 & 76.04 & 74.66 & 65.33 & 0.3 \\
\hline $\mathrm{C} 2$ & 13.85 & 13.78 & 13.78 & 14.03 & 14.41 & 13.91 & 0.29 \\
\hline C3 & 5.78 & 5.6 & 5.41 & 5.52 & 5.99 & 7 & 0.47 \\
\hline IC4 & 0.85 & 0.8 & 0.74 & 0.74 & 0.84 & 1.26 & 0.19 \\
\hline NC4 & 1.96 & 1.83 & 1.66 & 1.63 & 1.88 & 3.18 & 0.67 \\
\hline IC5 & 0.53 & 0.48 & 0.4 & 0.37 & 0.43 & 1.11 & 0.55 \\
\hline NC5 & 0.71 & 0.63 & 0.51 & 0.46 & 0.54 & 1.59 & 1.01 \\
\hline FC6 & 0.95 & 0.79 & 0.57 & 0.45 & 0.51 & 2.66 & 4.15 \\
\hline $\mathrm{C07}$ & 0.5 & 0.4 & 0.26 & 0.19 & 0.2 & 1.53 & 4.3 \\
\hline $\mathrm{C08}$ & 0.36 & 0.26 & 0.14 & 0.08 & 0.08 & 1.08 & 8.44 \\
\hline C09 & 0.25 & 0.16 & 0.07 & 0.03 & 0.03 & 0.61 & 12.59 \\
\hline C10 & 0.18 & 0.1 & 0.03 & 0.01 & 0.01 & 0.26 & 14.23 \\
\hline C11 & 0.13 & 0.06 & 0.02 & 0.01 & 0 & 0.1 & 13 \\
\hline C12 & 0.09 & 0.04 & 0.01 & 0 & $\overline{0}$ & 0.03 & 10.55 \\
\hline C13 & 0.07 & 0.02 & 0 & 0 & $\overline{0}$ & 0.01 & 8.02 \\
\hline C14 & 0.05 & 0.01 & 0 & 0 & $\overline{0}$ & 0 & 5.92 \\
\hline C15 & 0.03 & 0.01 & 0 & 0 & $\overline{0}$ & 0 & 4.31 \\
\hline C16 & 0.02 & 0 & 0 & 0 & 0 & 0 & 3.12 \\
\hline C17 & 0.02 & 0 & 0 & 0 & 0 & 0 & 2.24 \\
\hline C18 & 0.01 & $\overline{0}$ & 0 & 0 & $\overline{0}$ & 0 & 1.61 \\
\hline C19 & 0.01 & 0 & 0 & 0 & 0 & 0 & 1.15 \\
\hline $\mathrm{C} 20$ & 0.01 & 0 & 0 & 0 & $\overline{0}$ & 0 & 0.83 \\
\hline \multirow[t]{2}{*}{$\overline{\mathrm{C} 21+}$} & 0.02 & 0 & 0 & 0 & $\overline{0}$ & 0 & 2.06 \\
\hline & & & & & & & \\
\hline \multicolumn{8}{|c|}{ Equilibrium Gas } \\
\hline Z-Factor & 0.6771 & 0.6742 & 0.7272 & 0.8154 & 0.8859 & 0.9946 & \\
\hline \multirow{2}{*}{\multicolumn{8}{|c|}{ Gas Produced }} \\
\hline & & & & & & & \\
\hline \multicolumn{8}{|c|}{ cum. Mole $\%$ of } \\
\hline \multirow[t]{2}{*}{ original fluid } & & 20.75 & 45.37 & 68.96 & 82.85 & 98.91 & \\
\hline & & & & & & & \\
\hline \multicolumn{8}{|c|}{ Liquid, vol\% of } \\
\hline \multirow[t]{3}{*}{ original fluid } & & 4.63 & 6.28 & 5.44 & 4.45 & 1.62 & \\
\hline & & & & & & & \\
\hline & & & & & & & \\
\hline \multicolumn{2}{|c|}{ MW of components } & & & & & & \\
\hline 1 to 24 & & 23.0 & 22.0 & 21.7 & 22.1 & 28.7 & 155.1 \\
\hline
\end{tabular}

Table 4.5. Constant Volume Depletion Component Analysis 
Constant Composition Expansion Calculation

Summary of Constant Composition Expansion at 120 degrees $F$

\begin{tabular}{|c|c|c|c|c|c|c|c|c|c|c|c|c|}
\hline & p,psia & relative & oil & gas & gas & IFT & liquid & $\mathbf{Y}$ & sin.phase & oil & oil & gas \\
\hline & & tot vol & vis,cp & vis,cp & Z-factor & dyne/cm & vol \% & function & oil compr & Z-factor & density & density \\
\hline & & & & & & & of $\mathrm{CV}$ & & (1/psia) & & (Ib/ft3) & (Ib/ft3) \\
\hline & & & & & & & & & & & & \\
\hline 1 & 5014.7 & 0.7192 & & 0.0455 & 0.8990 & & 0 & 21.699 & & & & \\
\hline 2 & 4514.7 & 0.7521 & & 0.0422 & 0.8464 & & 0 & 20.749 & & & & \\
\hline 3 & 4014.7 & 0.7943 & & 0.0388 & 0.7949 & & 0 & 19.646 & & & & \\
\hline 4 & 3514.7 & 0.8510 & & 0.0353 & 0.7456 & & 0 & 18.338 & & & & \\
\hline 5 & 3014.7 & 0.9321 & & 0.0315 & 0.7004 & & 0 & 16.744 & & & & \\
\hline 6 & 2904.7 & 0.9549 & & 0.0306 & 0.6914 & & 0 & 16.343 & & & & \\
\hline 7 & 2814.7 & 0.9754 & & 0.0299 & 0.6844 & & 0 & 15.999 & & & & \\
\hline 8 & 2774.7 & 0.9851 & & 0.0295 & 0.6814 & & 0 & 15.841 & & & & \\
\hline \multirow[t]{4}{*}{9} & 2734.7 & 0.9953 & & 0.0292 & 0.6784 & & 0 & 15.680 & & & & \\
\hline & & & & & & & & & & & & \\
\hline & 2716.48 & 1.0000 & 0.0980 & 0.0290 & 0.6771 & 0.2233 & 0.0000 & & & 0.6768 & 15.60 & 32.638 \\
\hline & & & & & & & & & & & & \\
\hline 10 & 2714.7 & 1.0005 & 0.0979 & 0.0290 & 0.6770 & 0.2238 & 0.0092 & 1.2792 & & 0.6762 & 15.59 & 32.629 \\
\hline 11 & 2614.7 & 1.0313 & 0.0943 & 0.0279 & 0.6723 & 0.2574 & 0.5919 & 1.2437 & & 0.6431 & 15.03 & 32.190 \\
\hline 12 & 2514.7 & 1.0665 & 0.0918 & 0.0267 & 0.6693 & 0.3041 & 1.2773 & 1.2071 & & 0.6141 & 14.41 & 31.889 \\
\hline 13 & 2314.7 & 1.1527 & 0.0902 & 0.0242 & 0.6683 & 0.4553 & 2.6587 & 1.1367 & & 0.5645 & 13.04 & 31.702 \\
\hline 14 & 2124.7 & 1.2590 & 0.0916 & 0.0218 & 0.6738 & 0.6962 & 3.6225 & 1.0753 & & 0.5224 & 11.66 & 31.912 \\
\hline 15 & 2014.7 & 1.3342 & 0.0934 & 0.0206 & 0.6796 & 0.8918 & 3.9559 & 1.0424 & & 0.4990 & 10.85 & 32.160 \\
\hline 16 & 1714.7 & 1.6074 & 0.1021 & 0.0176 & 0.7042 & 1.6938 & 4.0903 & 0.9619 & & 0.4367 & 8.70 & 33.180 \\
\hline 17 & 1414.7 & 2.0290 & 0.1160 & 0.0153 & 0.7396 & 2.9776 & 3.4519 & 0.8942 & & 0.3747 & 6.73 & 34.574 \\
\hline 18 & 1234.7 & 2.3970 & 0.1273 & 0.0142 & 0.7648 & 4.0134 & 2.8937 & 0.8591 & & 0.3370 & 5.64 & 35.547 \\
\hline 19 & \begin{tabular}{|l|}
974.7 \\
\end{tabular} & 3.1944 & 0.1485 & 0.0131 & 0.8056 & 5.8850 & 2.0376 & 0.8143 & & 0.2810 & 4.21 & 37.106 \\
\hline 20 & 879.7 & 3.6117 & 0.1580 & 0.0128 & 0.8217 & 6.6817 & 1.7361 & 0.7995 & & 0.2598 & 3.73 & 37.720 \\
\hline
\end{tabular}

Table 4.6. Constant Composition Expansion Analysis

Constant Composition Expansion Calculation Summary of Constant Composition Expansion at 120 degrees $F$

Results after Regression

Saturation Pressure $=2716.48$ psia

\begin{tabular}{|c|c|}
\hline Component & Feed,\% \\
\hline N2 & 0.410 \\
\hline C1 & 73.220 \\
\hline C2 & 13.850 \\
\hline C3 & 5.780 \\
\hline IC4 & 0.850 \\
\hline NC4 & 1.960 \\
\hline IC5 & 0.530 \\
\hline NC5 & 0.710 \\
\hline FC6 & 0.950 \\
\hline C07 & 0.501 \\
\hline C08 & 0.357 \\
\hline C09 & 0.254 \\
\hline C10 & 0.181 \\
\hline C11 & 0.129 \\
\hline C12 & 0.092 \\
\hline C13 & 0.065 \\
\hline C14 & 0.047 \\
\hline C15 & 0.033 \\
\hline C16 & 0.024 \\
\hline C17 & 0.017 \\
\hline C18 & 0.012 \\
\hline C19 & 0.009 \\
\hline C20 & 0.006 \\
\hline C21+ & 0.015 \\
\hline
\end{tabular}

Table 4.7. Constant Composition Expansion Analysis after Regression 


\section{Constant volume depletion calculation Results after regression}

Summary of Cumulative Recovery during Constant Volume Depletion at $120 \mathrm{deg} F$

\begin{tabular}{|c|c|c|c|c|c|c|c|c|}
\hline \multicolumn{3}{|c|}{ Initial Depletion Pressure, psia } & 2716.5 & 2114.7 & 1514.7 & 914.7 & 514.7 & 14.7 \\
\hline & & & & & & & & \\
\hline \multicolumn{2}{|c|}{ Well Stream MSCF } & & 1000 & 207.49 & 453.69 & 689.64 & 828.47 & 989.13 \\
\hline & & & & & & & & \\
\hline \multicolumn{9}{|c|}{\begin{tabular}{|l|l|} 
Separator & Recovery \\
\end{tabular}} \\
\hline \multicolumn{2}{|c|}{ Stock Tank Liquid bbl } & & 39.046 & 5.134 & 8.308 & 10.216 & 11.486 & 25.074 \\
\hline \multirow{2}{*}{\multicolumn{3}{|c|}{ Primary Separator Gas MSCF }} & & & & & & \\
\hline & & & 913.344 & 194.413 & 431.441 & 661.121 & 795.032 & 921.162 \\
\hline \multicolumn{3}{|c|}{ Second Stage Gas MSCF } & 24.775 & 3.827 & 6.581 & 8.531 & 10.134 & 20.135 \\
\hline & & & & & & & & \\
\hline \multicolumn{2}{|c|}{ Stock Tank Gas MSCF } & & 24.486 & 3.933 & 6.897 & 9.08 & 10.95 & 20.874 \\
\hline & & & & & & & & \\
\hline \multirow[t]{2}{*}{ GOR } & MSCF/bbl & & 24.65 & 39.38 & 53.55 & 66.44 & 71.05 & 38.37 \\
\hline & & & & & & & & \\
\hline \multirow[t]{2}{*}{$\mathrm{m} 3 / \mathrm{m} 3$} & & & 4391 & 7013 & 9538 & 11834 & 12655 & 6835 \\
\hline & & & & & & & & \\
\hline & & & & & & & & \\
\hline \multirow[t]{2}{*}{ Separator } & Conditions & & & & & & & \\
\hline & & & & & & & & \\
\hline \multirow[t]{3}{*}{1} & Pressure, & psia & 514.7 & 514.7 & 514.7 & 514.7 & 514.7 & 514.7 \\
\hline & \multicolumn{2}{|c|}{ Temperature, deg F } & 54 & 54 & 54 & 54 & 54 & 54 \\
\hline & & & & & & & & \\
\hline 2 & Pressure, & psia & 114.7 & 114.7 & 114.7 & 114.7 & 114.7 & 114.7 \\
\hline & \multicolumn{2}{|c|}{ Temperature, $\operatorname{deg} \mathrm{F}$} & 54 & 54 & 54 & 54 & 54 & 54 \\
\hline & & & & & & & & \\
\hline 3 & Pressure, & psia & 14.7 & 14.7 & 14.7 & 14.7 & 14.7 & 14.7 \\
\hline & \multicolumn{2}{|c|}{ Temperature, deg F } & 60 & 60 & 60 & 60 & 60 & 60 \\
\hline
\end{tabular}

Table 4.8. Constant Volume Depletion Analysis after Regression

The EOS models used in earlier studies compared and predicted fluid properties very similar to the properties determined from the regression output from the Winprop EOS model (Figure 4.25). Only the oil saturation property from the EOS models are inconsistent with the laboratory values. However, since the earlier studies agree with the later WinProp calculated values for the oil saturation properties over the pressure range it is believed the gas and liquid samples recombined in the laboratory data might not been representative of the original reservoir fluid. This is shown that the lab data of oil saturation prediction is shows much less retrograde condensate drop out than the other EOS models described above. It was concluded that the lab sample performed in 1979 must have been from a leaner mixture. 

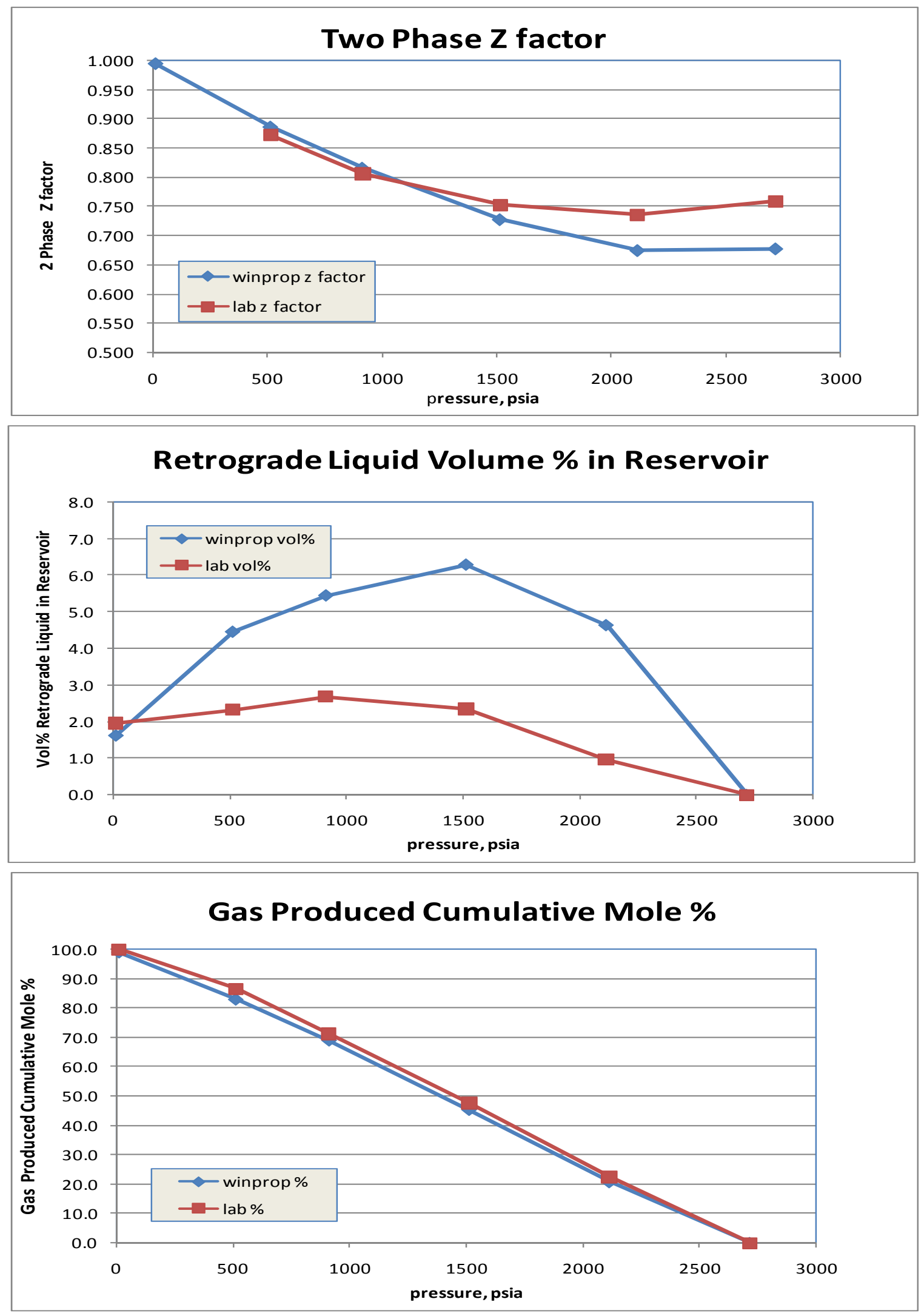

Figure 4.25. WinProp EOS Fluid Properties Analysis Graphs 
The retrograde Dewpoint saturation pressure was shown in the original PR-EOS studies to be 2714.7 psia. The WinProp after regression calculated saturation pressure was 2716.483 psia and 120.0 deg F using the Peng-Robinson Equations of State. This matched well with the original PR-EOS studies described in the first part of the results section.

The graph in Figure 4.26 shows the results of the WinProp phase behavior simulator final Constant Volume Depletion (CVD) Regression Summary compared with the original laboratory results. The WinProp data on the \% Liquid Volume in the reservoir compares favorably with the original PR-EOS solution shown in Figure 4.27. The WinProp simulator data on the \% Gas Produced from the reservoir also compares favorably with the original PR-EOS solution for \% Gas In Place Produced shown in Figure 4.28.

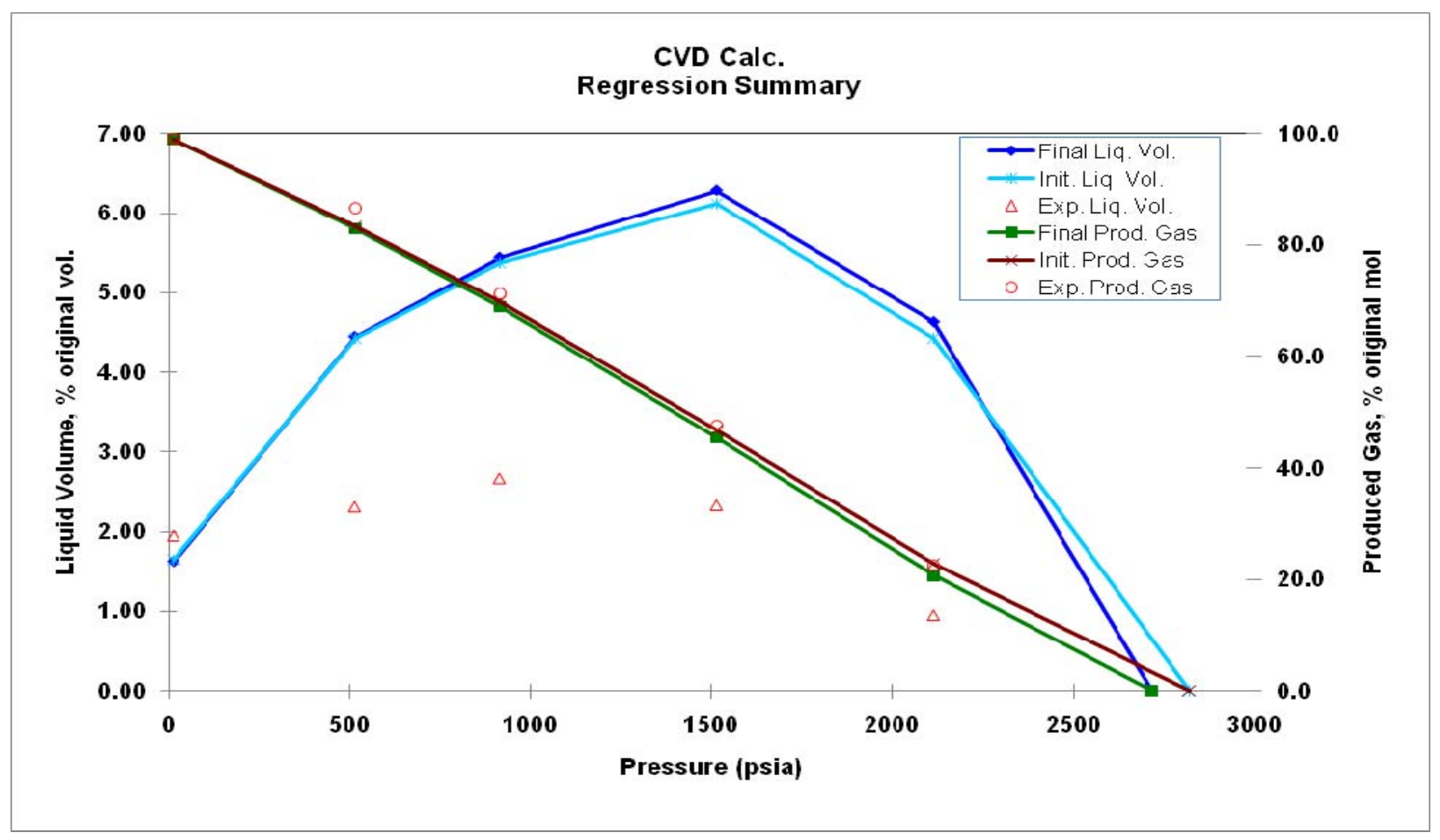

Figure 4.26. WinProp CVD Calc Regression Summary 


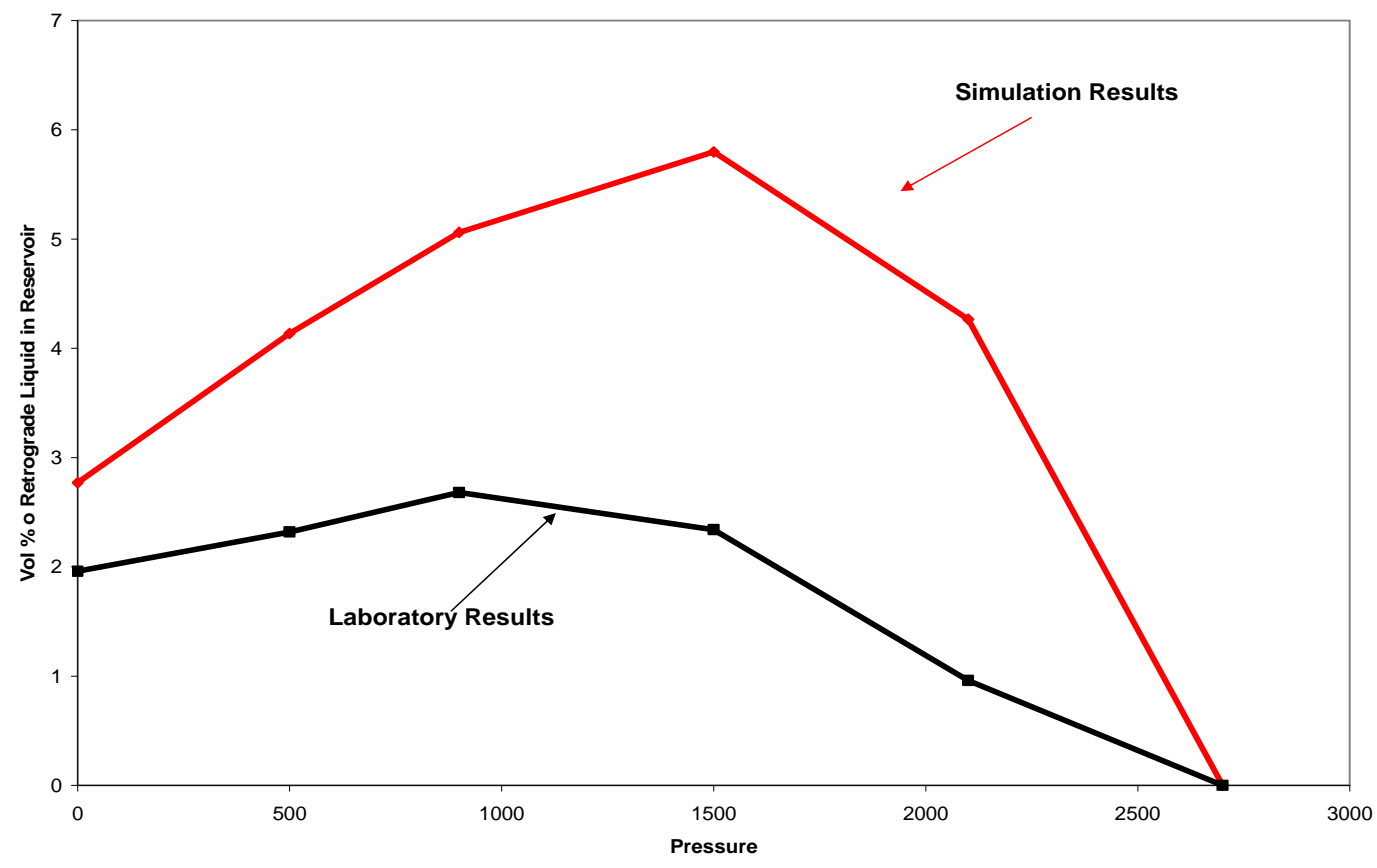

Figure 4.27. Original PR-EOS Vol \% Liquid in Reservoir

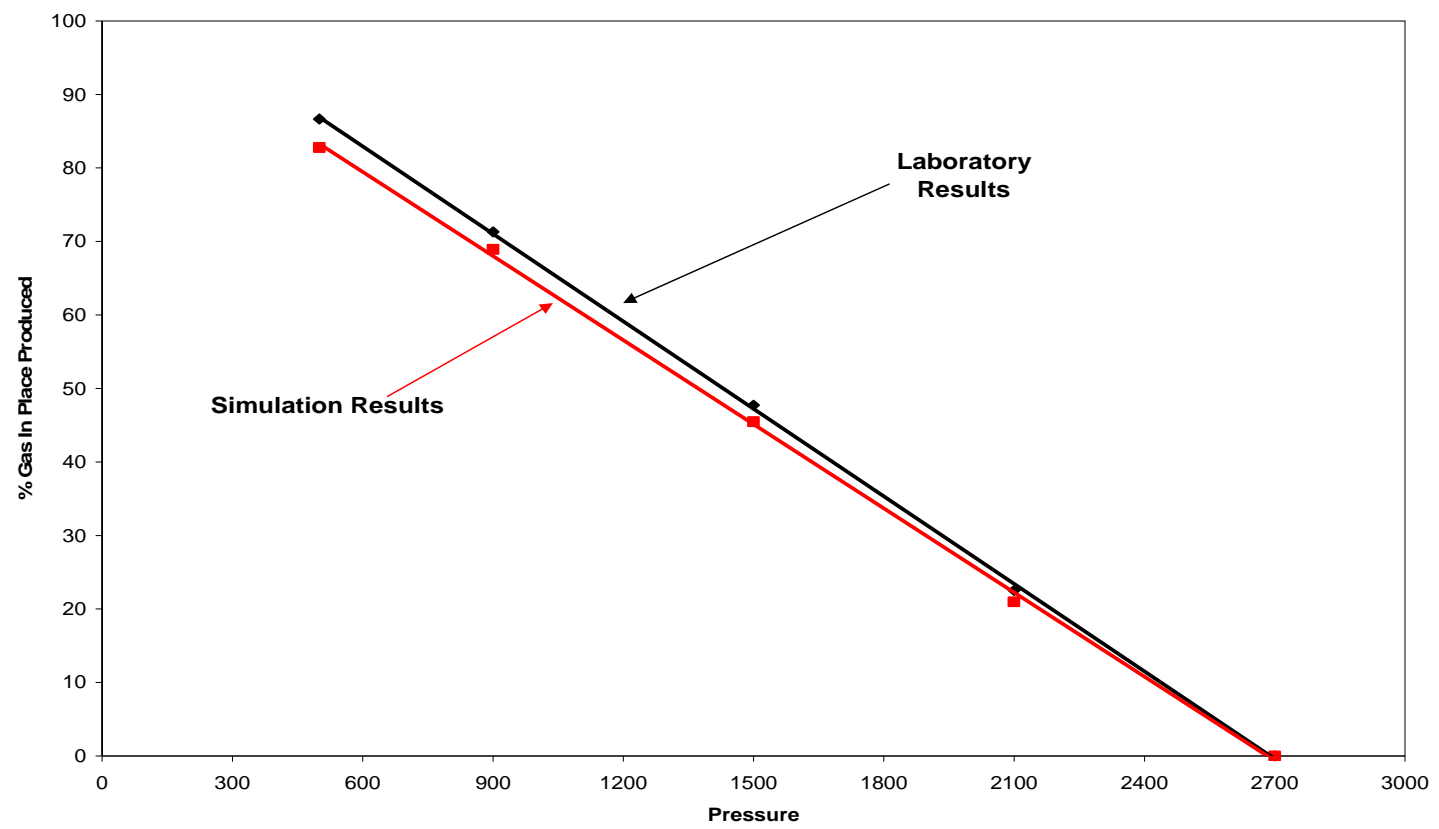

Figure 4.28. Original PR-EOS \% Gas in Reservoir 
The laboratory results for the \% Liquid Volume in the reservoir are lower over the range of pressures during depletion both for the original PR-EOS ${ }^{1}$ and the WinProp results using the PR-EOS Case 33 Composition. One could conclude that the reason that this happens is because of the factors Whitson et al(1983)explained in evaluating constant volume depletion (CVD) data. The PR-EOS(Aminian 1983) and the WinProp results for the \% Gas in Reservoir matched the laboratory data initially at the original saturation pressure. As the pressure decreased during the depletion phase, the \% Gas in Reservoir predicted by the laboratory analysis was higher than that predicted by the EOS simulation models. This would have resulted because the EOS models both overestimated the \% oil saturation during the depletion phase of production.

In Appendix under Section results of the WinProp output from the Constant Volume Depletion(CVD) and Constant Composition Expansion(CCE) experiments before the regression and after the regression analysis can be found. Oil compressibility,viscosity and relative volume, liquid volume, $\mathrm{z}$ factor and gas density.

\section{Material Balance Analysis}

The production history and predicted 2-phase z-factors by the EOS simulator as well as two phase z-factors from laboratory study were utilized to generate material balance plots (P/z vs. Cumulative Wet Gas Production). Figure 4.29 illustrates this plot for both sets of 2-phase zfactors. Same values for IGIP were obtained using both sets of 2-phase z-factors. It should be noted that even though the two set of z-factors were different, their similar trend versus pressure resulted in the same values of IGIP. This is mainly due to relatively small liquid volumes in the reservoir. An Equation of State model was used to simulate the primary production where an estimate of the two-phase z-factor could be obtained to construct a p/z versus cumulative gas production to determine the actual original gas in place and remaining wet gas reserves. Table 4.9 and 4.10 shows the regression parameters indicating an original wet gas in place of 8.056 BCF. 


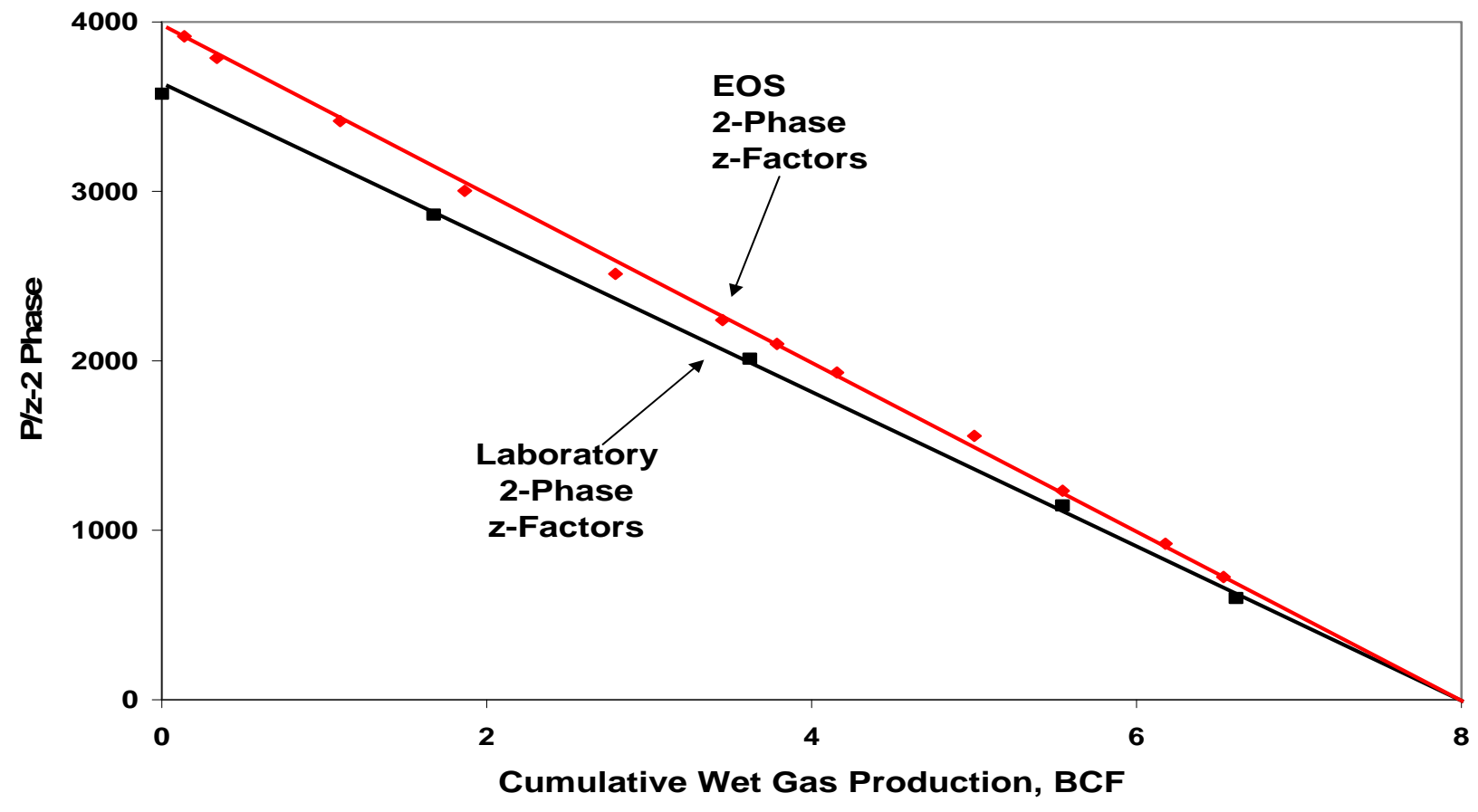

Figure 4.29. EOS Simulation of $P / Z$ versus Cumulative Wet Gas production

EOS DATA-MATERIAL BALANCE

\begin{tabular}{|c|c|c|c|c|c|c|}
\hline Date & SIWHP & BHP & BHP & EOS-RUN33-2-Z & $\mathrm{P} / \mathrm{z}$ & CUM \\
\hline & psig & psig & psia & 0.681738 & & BCF \\
\hline $8 / 9 / 1979$ & 2256 & 2642 & 2656.7 & 0.678524 & 3915.4 & 0.138 \\
\hline 10/14/1979 & 2169 & 2542 & 2556.7 & 0.674977 & 3787.8 & 0.338 \\
\hline $7 / 9 / 1980$ & 1938 & 2275 & 2289.7 & 0.670568 & 3414.6 & 1.099 \\
\hline $3 / 3 / 1981$ & 1725 & 2010 & 2024.7 & 0.67411 & 3003.5 & 1.865 \\
\hline $3 / 11 / 1982$ & 1475 & 1711 & 1725.7 & 0.686463 & 2513.9 & 2.793 \\
\hline $3 / 1 / 1983$ & 1320 & 1546 & 1560.7 & 0.696374 & 2241.2 & 3.452 \\
\hline $1 / 3 / 1984$ & 1250 & 1460 & 1474.7 & 0.70219 & 2100.1 & 3.788 \\
\hline $8 / 10 / 1985$ & 1165 & 1356 & 1370.7 & 0.709628 & 1931.6 & 4.156 \\
\hline $5 / 24 / 1988$ & 968 & 1118 & 1132.7 & 0.727444 & 1557.1 & 5.003 \\
\hline 8/1/1990 & 785 & \begin{tabular}{l|l}
901 \\
\end{tabular} & 915.7 & 0.743002 & 1232.4 & 5.545 \\
\hline $8 / 31 / 1993$ & 600 & 680 & 694.7 & 0.75478 & 920.4 & 6.178 \\
\hline $7 / 25 / 1996$ & 459 & 533 & 547.7 & 0.756683 & 723.8 & 6.536 \\
\hline
\end{tabular}

Table 4.9. Material Balance Data 
SUMMARY OUTPUT

\begin{tabular}{|c|c|c|}
\hline \multicolumn{2}{|c|}{ Regression Statistics } & \\
\hline Multiple R & 0.999558321 & \\
\hline R Square & 0.999116838 & \\
\hline Adjusted R Square & 0.999028522 & \\
\hline Standard Error & 33.69951009 & \\
\hline Observations & 12 & \\
\hline & Coefficients & Standard Error \\
\hline Intercept & 3948.632237 & 18.47168123 \\
\hline X Variable 1 & -490.1318973 & 4.608133254 \\
\hline Gas In Place & 8.056264566 & \\
\hline $\mathbf{P i}$ & 2691.932644 & \\
\hline Initial Z-factor & 0.681738 & \\
\hline
\end{tabular}

Table 4.10: P/z against cumulative wet gas production(Material Balance Plot)

\section{Simulation of Storage Gas Cycling}

Figure 4.30 summarizes the predicted liquid yields for 3 storage cycles. In prediction of liquid yields, it was assumed the injected gas was completely mixed with the residue gas and liquid. The full mixing assumption is based on the fact the reservoir is highly porous and permeable and the liquid is completely immobile due to low saturation. The re-evaporation of the liquid is mainly caused by pressure increase. The injection well configurations are also such that the injected gas will contact the entire reservoir and therefore the residue gas and injected gas will be fully mixed. However, it is important to evaluate the results of first storage withdrawal cycle to verify the degree of mixing and accuracy of the predicted liquid yields.

These results indicate that when the lean storage gas comes into contact with the wet residue gas and condensate subsequent gas cycling or re-pressurization and revaporization less liquids are produced and recovered at the surface. 


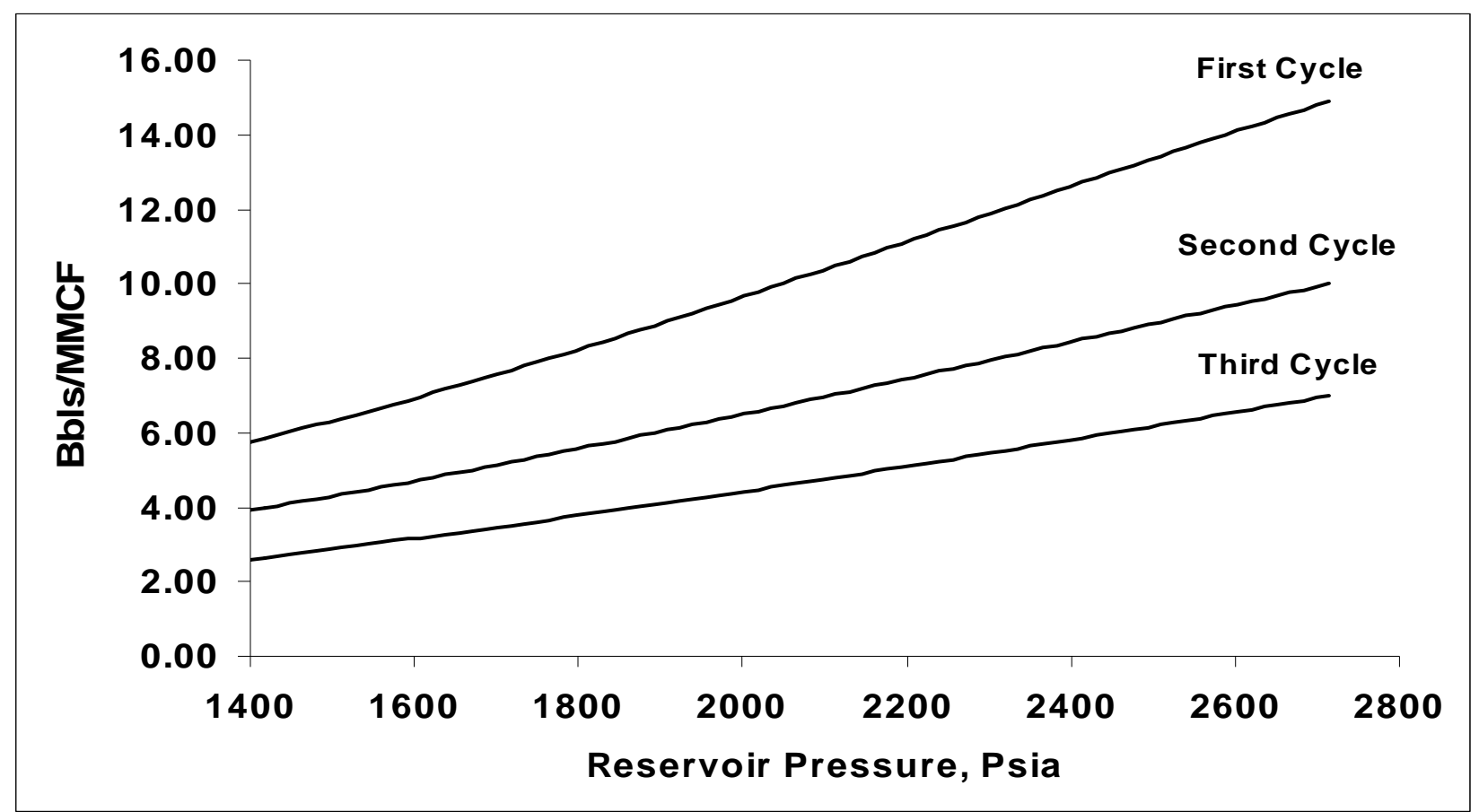

Figure 4.30: Predicted Storage Liquid Yields

This part of the study concluded that the Peng -Robinson(PR) Equation of State as stated by Aminian, K.: "Phase-Equilibrium and Reservoir Depletion Calculations on PC Using the PengRobinson Equation of State, SPE Computer Applications, May-June 1989, 20. can be used to predict the phase behavior of gas condensate reservoir accurately. Also, PR-EOS can be used to predict the phase behavior of a condensate reservoir after conversion to storage if the level of mixing can be estimated. 


\section{COMPOSITIONAL MODEL DEVELOPMENT}

The steps required in the development of a representative reservoir model usually become very difficult because of the difficulty in working with several sets of data from varying sources as well as data that that must be inferred or where formulas have to be developed. Many times these steps must be repeated until a spatial representation can be obtained from a reservoir property and areal geographic view.

The reservoir model development can be broken down into the following steps:

- develop grid size and block dimensioning

- import geologic characterization of reservoir

- apply porosity scheme from net pay data

- $\quad$ establish relevant pore volume representation for field

- use core derived porosity permeability relationship to develop perm formulas

The CMG model reservoir description was constructed using the Geographix output files described above. The $x$ and y horizontal grid size dimensions of 25 by 25 was determined to be adequate for the level of detailed geologic properties available. Also, the horizontal k vertical grid dimension we used a 5 layer description that we deemed adequate with the level of vertical geologic data available. The total number of grid blocks in the model was 3125 with the above dimensions of $25 \times 25 \times 5$. The $x$ and y dimensions were based on a grid block size of $80 \mathrm{ft}$ by $80 \mathrm{ft}$. The $\mathrm{k}$ height grid block size was approximately $40 \mathrm{ft}$. This gave a unit volume for each grid of $2.560 \times 10^{\wedge} 5 \mathrm{ft} 3$. The total acreage for the reef structure was estimated at approximately 100 acres. Figures $4.31,4.32,4.33$ and 4.34 show how the model in various different views. Figure 4.31 shows how the grid dimensioning was established. 
Grid Top (ft) 1979-05-01 Klayer: 1

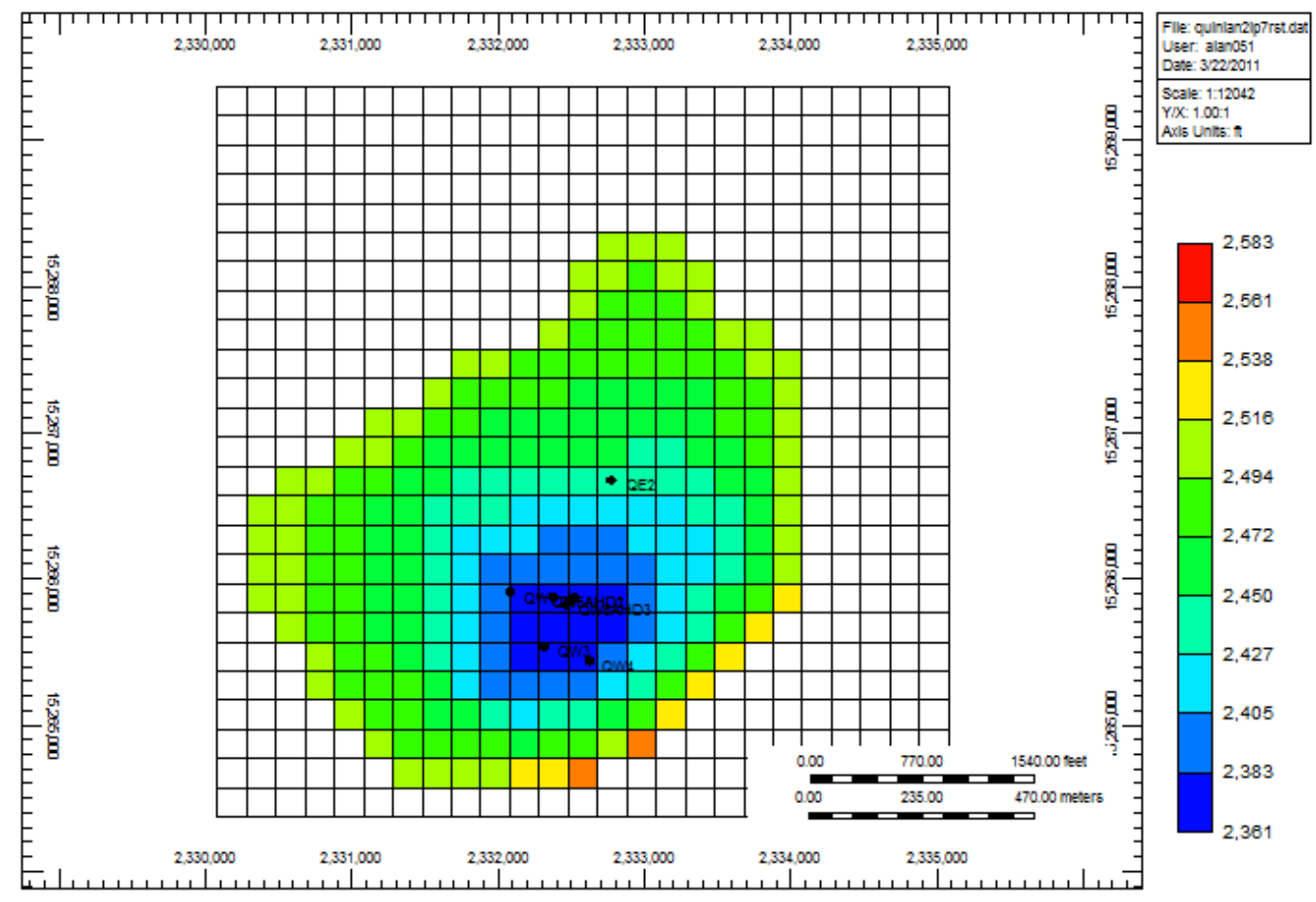

Figure 4.31. 2D IJ Areal Grid Dimension View

Grid Top (ft) 1979-05-01

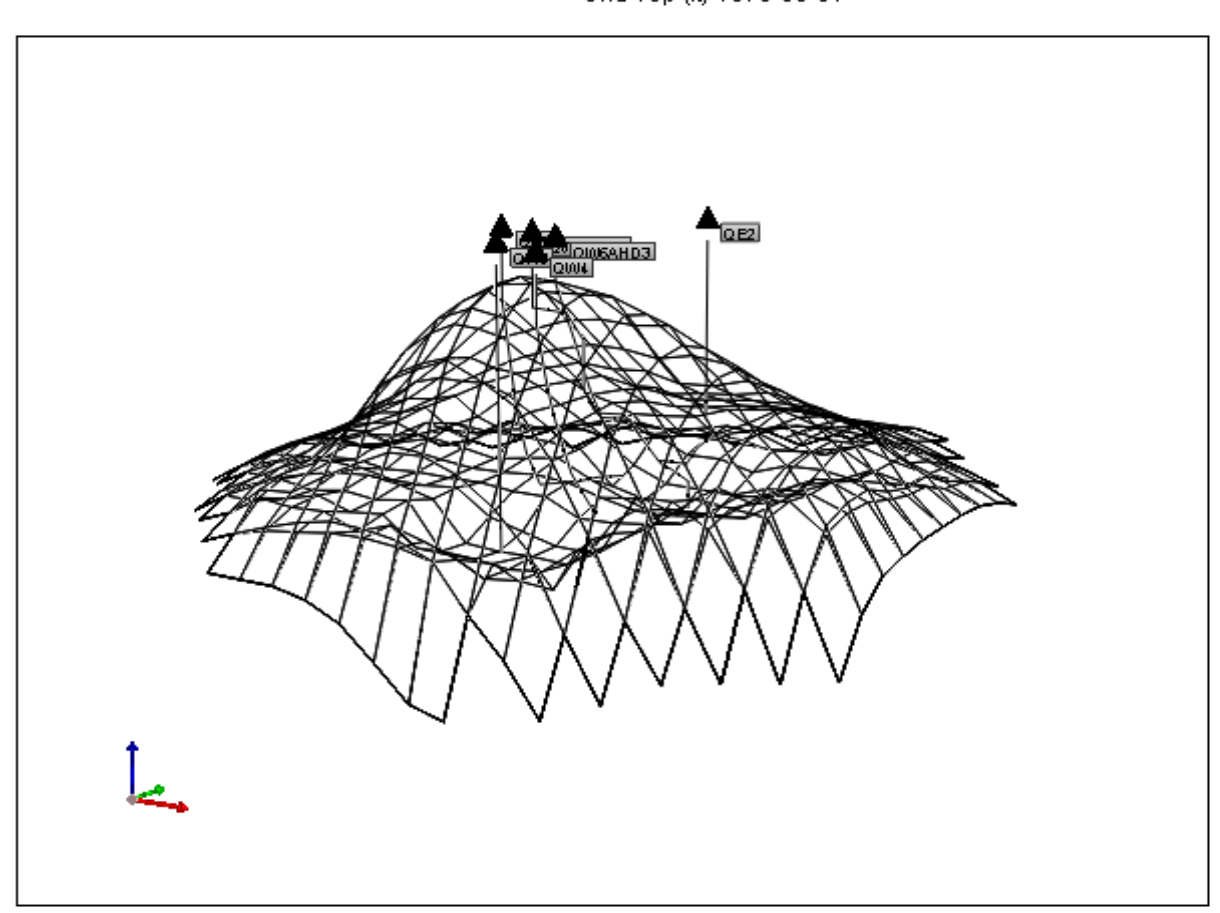

Ile:quilen2pirstdat User: abuss

$\mathrm{zx}: 9 \mathbf{m}: 1$

Figure 4.32. 3D IJK Areal Grid Dimension View 
Grid Top (ft) 1979-05-01 J layer: 17

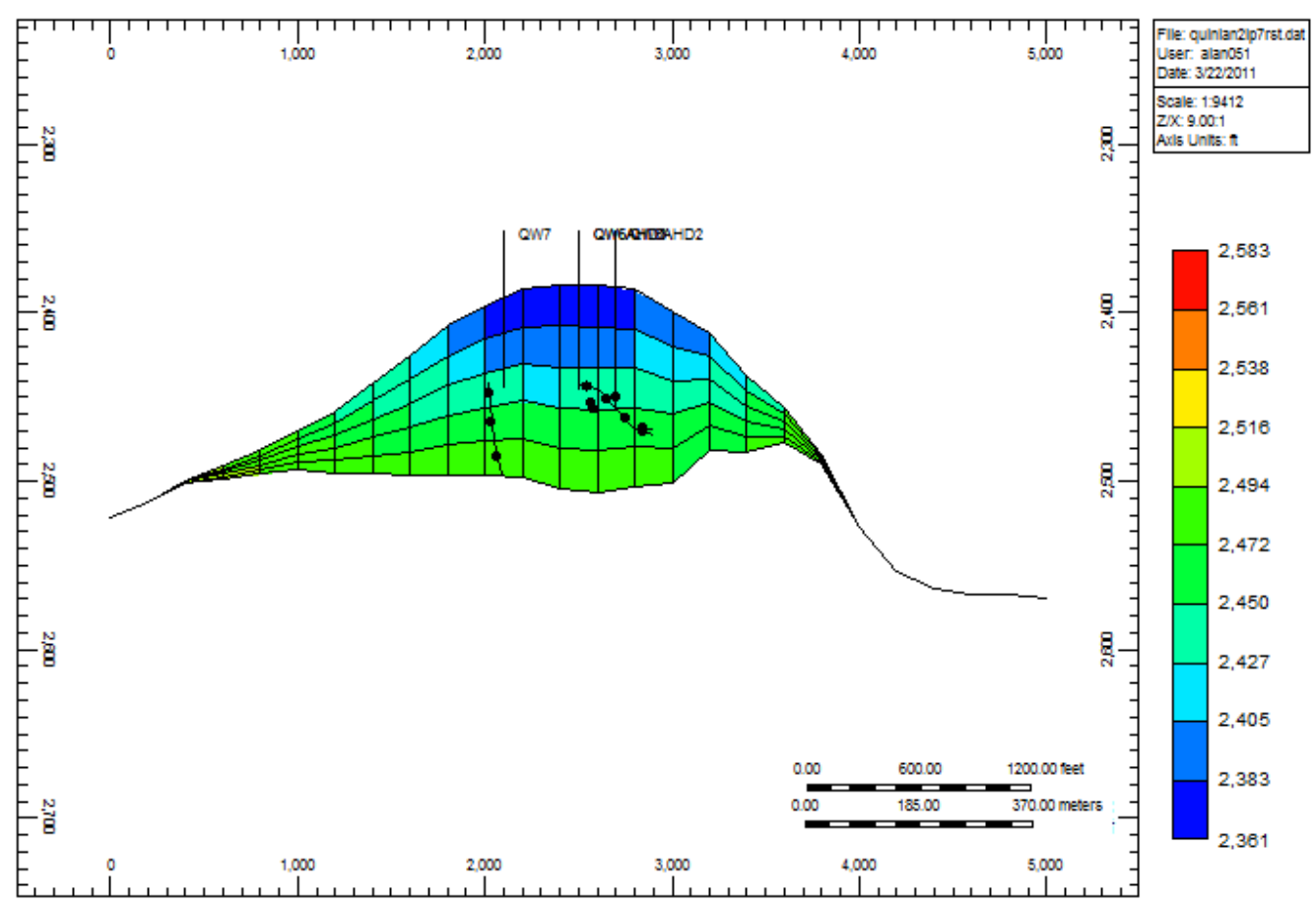

Figure 4.33. 2D J Layer:17 Cross Section View

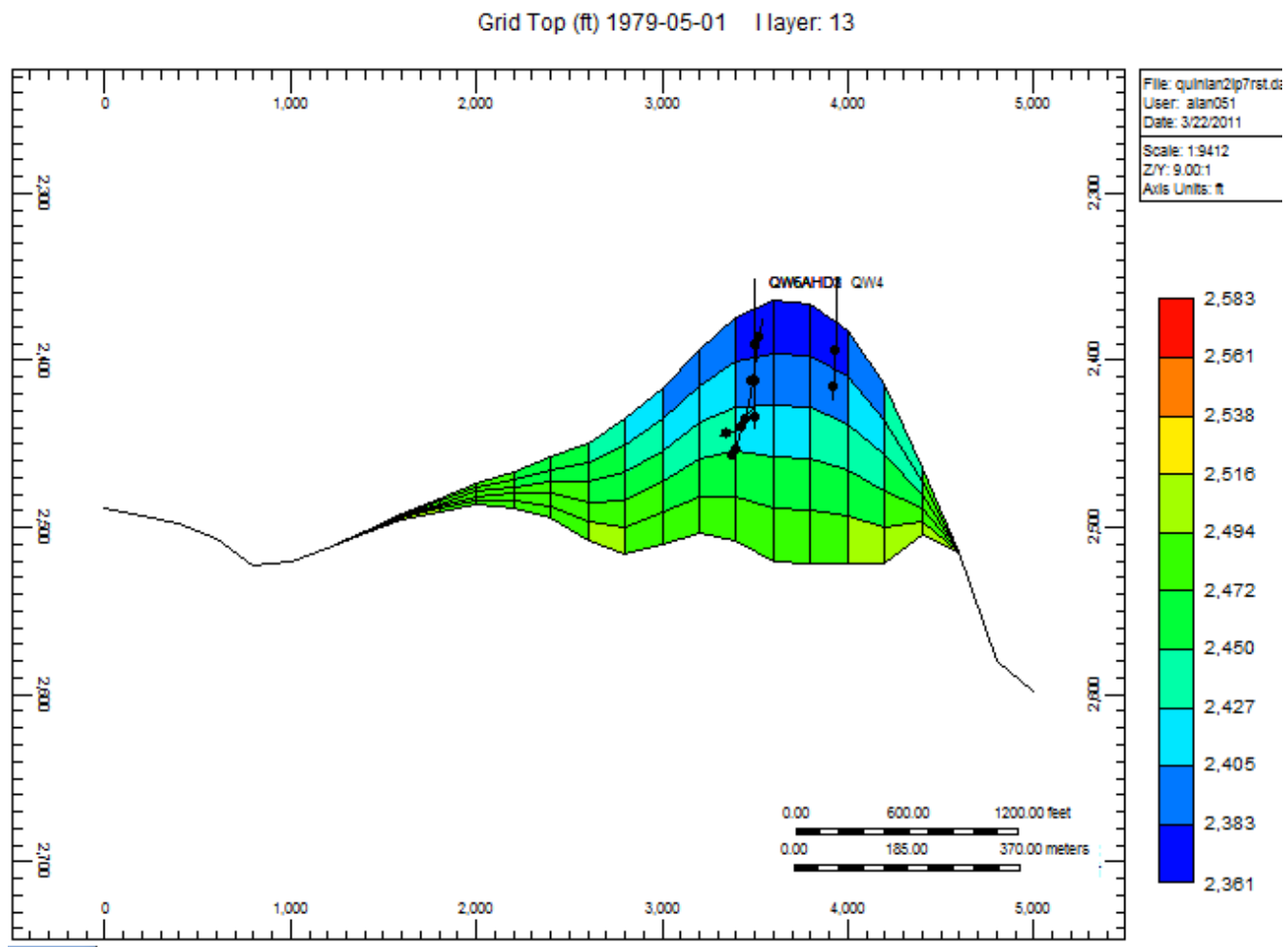

Figure 4.34. 2D I Layer:13 Cross Section View 
The following files were exported using the IsoMap export utility of the GeoGraphix Exploration System. File exported from layer (3DPAK) using the IsoMap export utilityof the GeoGraphix Exploration System.

- Onondaga Reef Net Thickness NETCON.DAT

- Onondaga Reef Lime -V Top ONONT.DAT

Figure 4.35 shows the 3-Dimensional Grid Map of the Reservoir (Subsea Elevation, ft) after imported from the Geographix files described above.

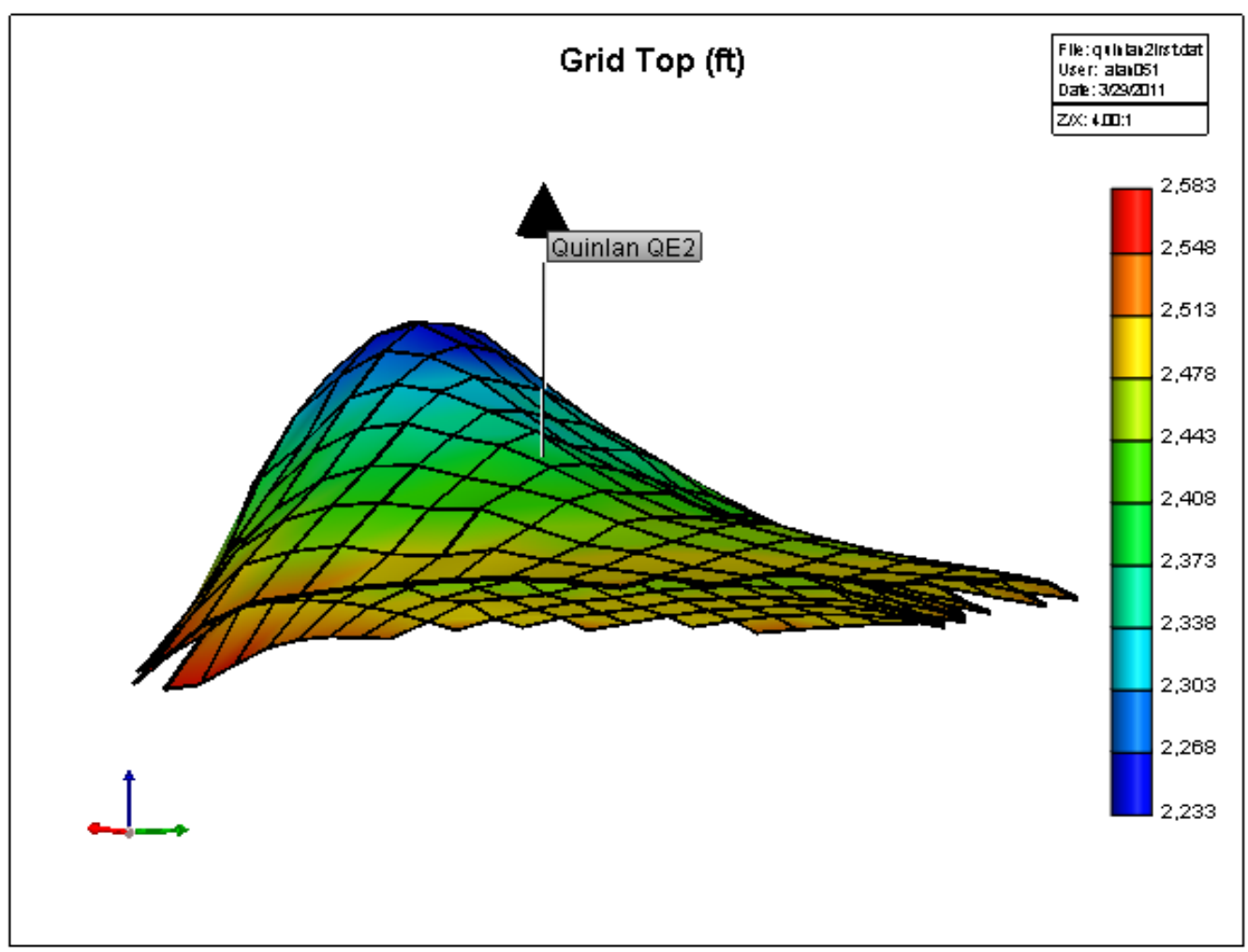

Figure 4.35. 3-Dimensional Grid Map of the Reservoir (Subsea Elevation, $\mathrm{ft}$ ) 
The porosity calculations were based on the Sonic transient times from the 3D seismic. The geographix system was used to process the seismic and develop a velocity pullup scheme from the slowing in low density high porosity limestone structures. The transient travel time were 709 to 730 millisecs or 21 millisecs difference between the top and bottom of the Onondoga limestone. This difference was multiply by the $13,000 \mathrm{ft} / \mathrm{sec}$ limestone sonic transient time to calculate a $273 \mathrm{ft}$ max net pay in the best part of the structure. A $\sim 8.2 \mathrm{~ms} / \mathrm{ft}$ value was used in converting 2-way travel time to footage. For example, QW-3 3D Seismic net value was 19 ms -> (730 ms (Reef Top) - $711 \mathrm{~ms}$ (Reef Net bottom)). Next, $19 \mathrm{~ms}$ x $8.2 \mathrm{~ms} / \mathrm{ft}=155.8^{\prime}$ (net).

The porosity was then based on a 2.55 cut off or 6-8\% Logs from QW3 were used to determine the 204 gross pay and $155 \mathrm{ft}$ net pay with $12.67 \%$ porosity. The Geographix system had no way to determine porosity. The only files used in the CMG reservoir model software were the net pay and vertical top of structure. Figure $\mathbf{4 . 3 6}$ shown below shows the interpretation of seismic data and the porosity scheme described above. There were three (3) distinct porosity zones identified and mapped; the highest or maximum porosity zone is everything greater including 70 $\mathrm{ft}$ of net pay, the next or intermediate porosity zone is everything between 20 and $70 \mathrm{ft}$ of net pay and the final or lowest porosity zone is from 0 to 20 feet of net pay.

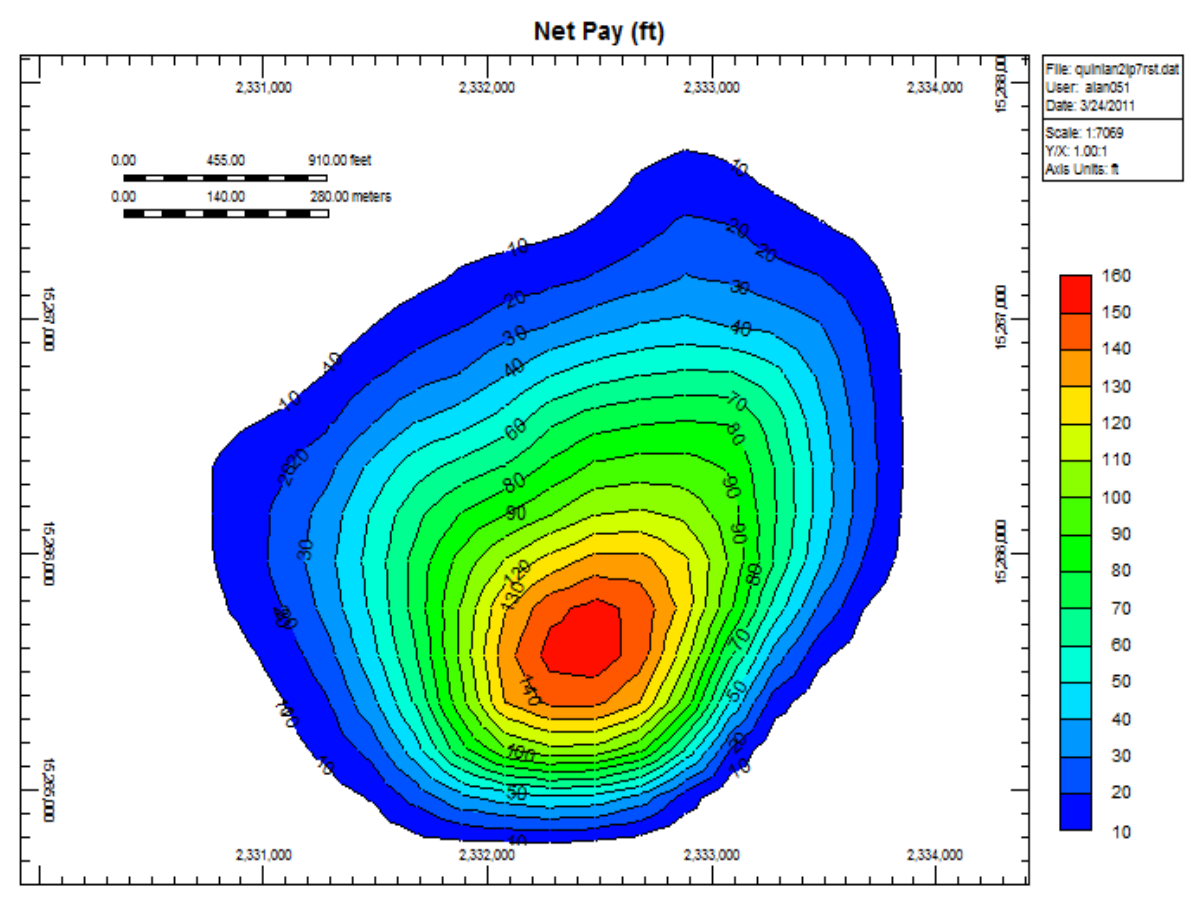

Figure 4.36. Net Isopach Map/Porosity Zones of Onondaga Formation 
The following relationships were developed based on the information provided in the Westport Geological services "Reservoir Characterization of the Onondaga Reef QW-3 Well, Quinlan Field Cattaraugus County, New York" Report No. RT-00-006 performed on March 8, 2000.Core data was used to build porosity- permeability cross plots. The extracted and Non Extracted data was used to construct cross-plots to develop formulas for the model. The following two figures show the cross plot Figure 4.37 shows the Core Derived Net Pay-Porosity Cross plot and Figure 4.38 shows the Core Derived Porosity-Permeability Cross plot:

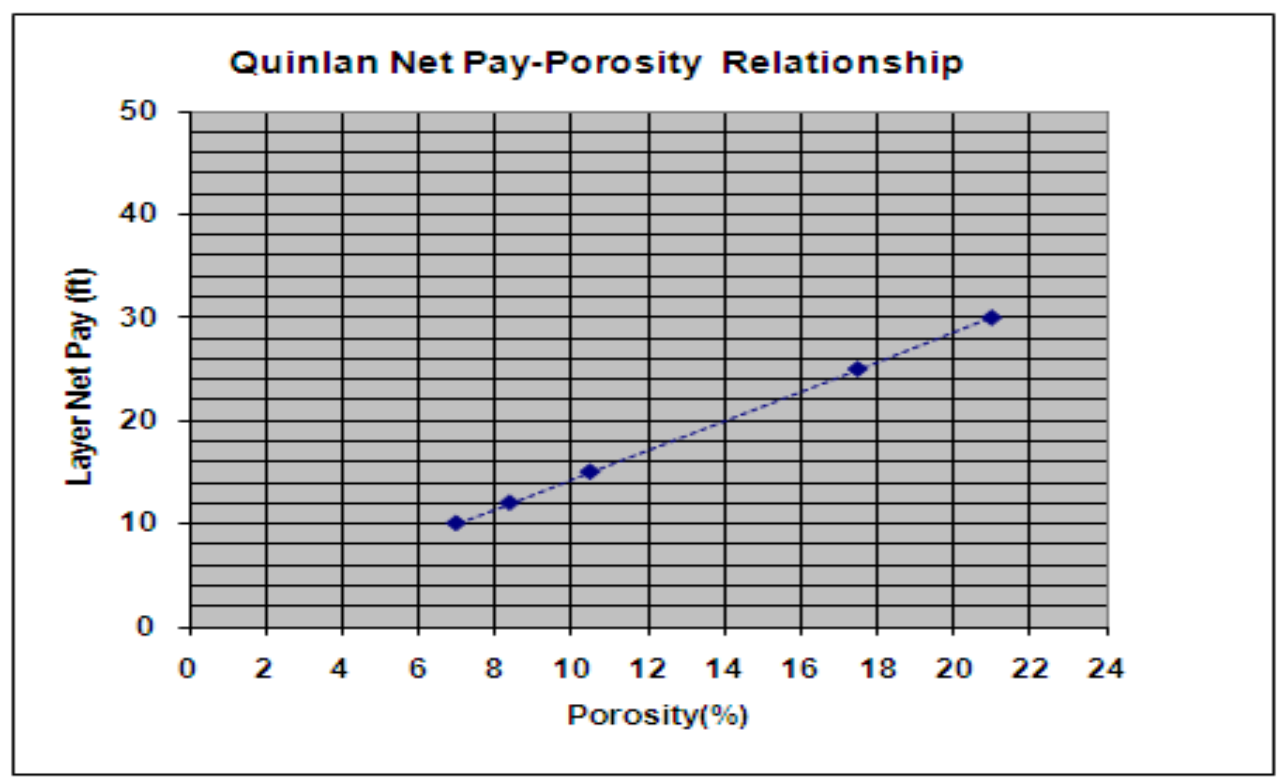

Figure 4.37. Core Derived Net Pay-Permeability Cross plot

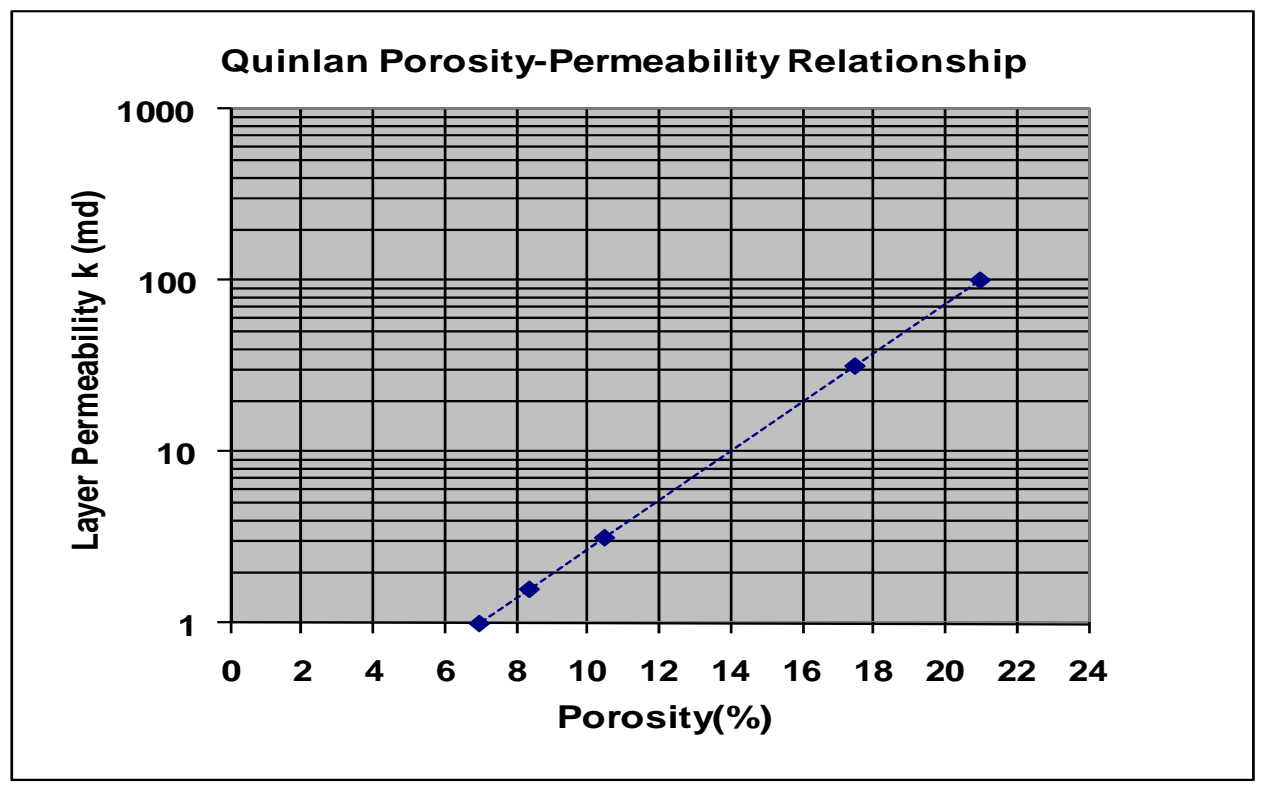

Figure 4.38. Core Derived Porosity-Permeability Cross plot 
Figure 4.39 below shows the porosity values for the reservoir used in the model:

Porosity (p.u.)

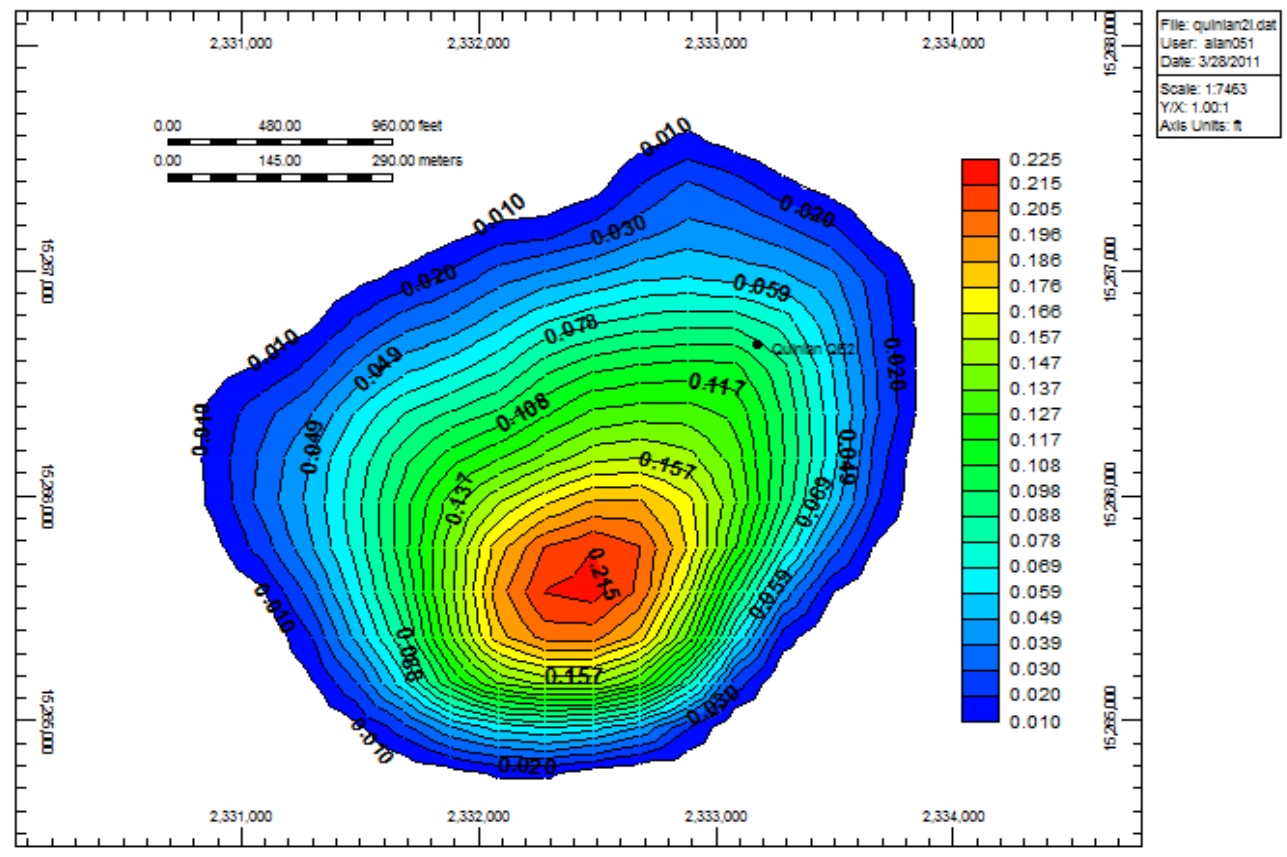

Figure 4.39. Porosity Zones of Onondaga Formation

The following data was generated from the CMG Builder application Rock Fluid section where the relative permeability tables were developed to apply to the entire model. All of the grids in the model used the same liquid- gas relative permeability curve..

Liquid- Gas Relative Permeability

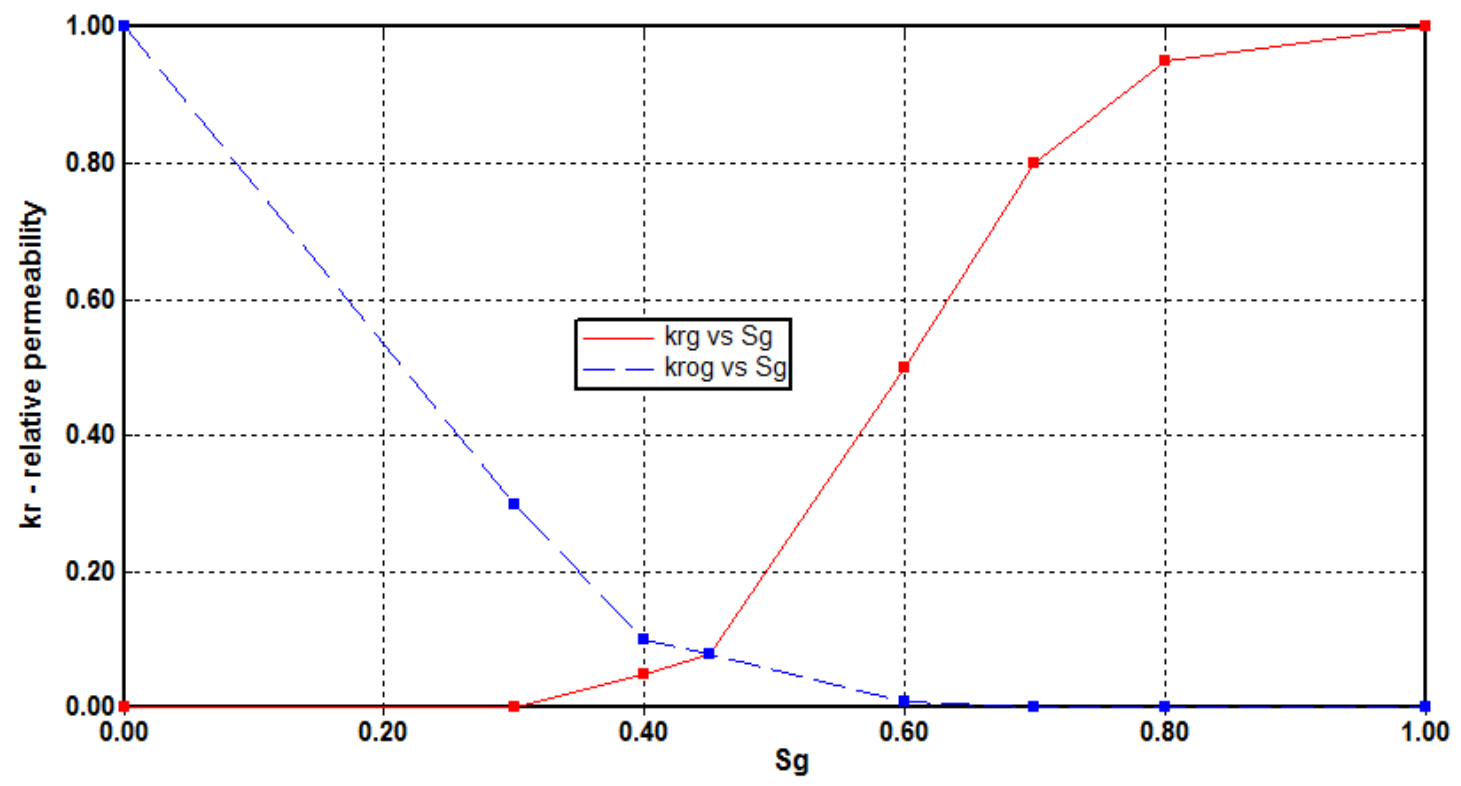

Figure 4.40. Liquid-Gas Relative Permeability Curve 
Figure 4.40 shows the liquid-gas relative permeability curves. Since this is a very lean condensate system with a very low oil saturation it is presumed that the affects of then liquids on gas flow will be very negligible.

The Three phase diagram used in the Rock-Fluid section is show below in Figure 4.41:
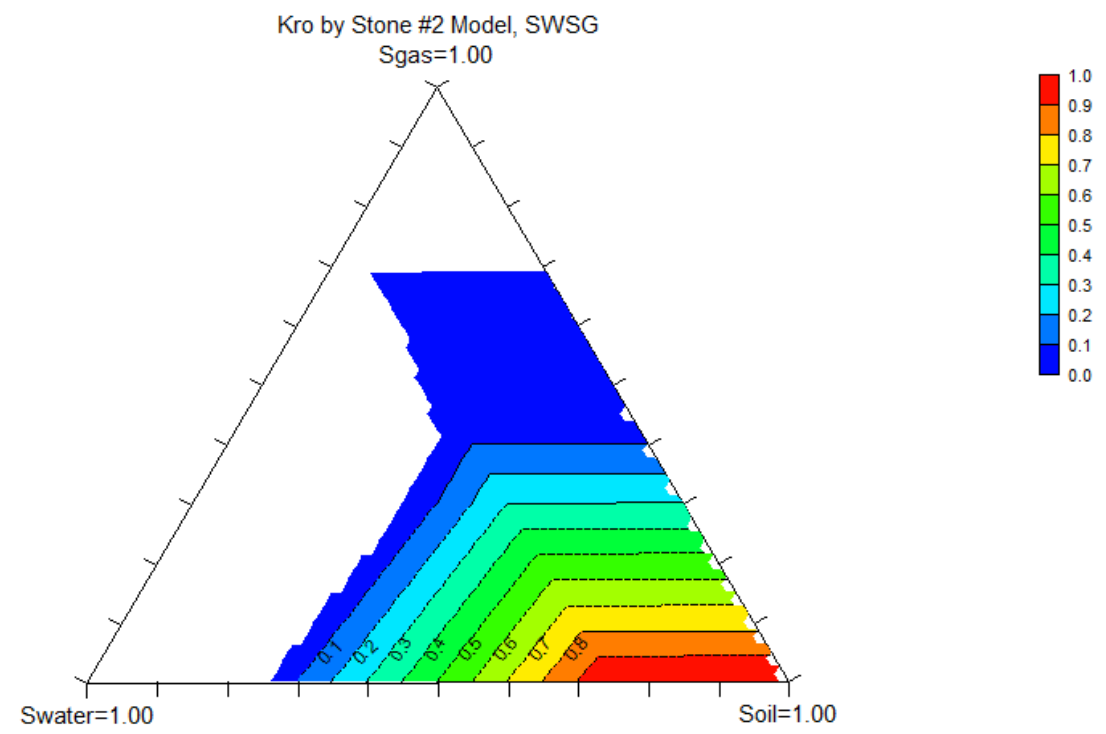

Figure 4.41. Three Phase Diagram for Fluid Description 


\section{HISTORY MATCHING DISCUSSION}

History matching and model calibrations are an important part of the Reservoir modeling work flow process. The history matching process was started after a reasonable description of the initial composition of the reservoir fluid was modeled and the reservoir properties characterization was completed.

The equation of state model was used to identify the characterization of the reservoir fluids at the beginning of the production phase of the reservoir. Since there was limited laboratory data on initial reservoir fluid this characterization focused on several key parameters for reservoir fluids.

The geologic description defined in the reservoir model was developed using existing seismic, core analysis and reservoir property log data. To define the reservoir size, reservoir engineering volumetric and material balance techniques were used to determine the reservoir model interpretation.

The cumulative monthly condensate production history shows that approximately 115,000 bbls were produced and recovered. The results of the original PVT fluid study, the recent Equation of State study and recent compositional reservoir simulation study all indicate that approximately one third of the original condensate liquid in place has been produced. It estimated that there between 180,000 and 230,000 remaining stock tank barrels in the reservoir. There was insufficient data to make an estimate of the volumetric initial gas in place because the only well drilled QE-2 only penetrated the top 20 feet making it difficult to estimate the reservoir pay thickness. There were some estimates of the gross pay thickness based on the earlier seismic data.

The initial estimates on the reservoir container size was approximately 10 BCF using the imported reservoir characterization files from Geographix software package. A couple of factors caused this and were corrected to arrive at the proper reservoir size. The reservoir model did not take into account any porosity cut off or net pay consideration. To account for this a porosity cut off of porosity $<2.1 \%$ was defined in the model reservoir model along with this applies to the individual layers and not the whole grid. 
After these adjustments were made the following descriptions were shown in the reservoir model:

$\begin{array}{ll}\text { Total Hydrocarbon Pore Volume } & 335195 \text { res } \mathrm{ft}^{\wedge} 3 \\ \text { Total Pore Volume } & 446927 \text { res ft^3 } \\ \text { Average Porosity } & 12.75 \% \\ \text { Original Gas In Place (OGIP) } & 80545200 \mathrm{std} \mathrm{ft}^{\wedge} 3 \\ \text { Original Oil In Place (OOIP) } & 117387 \mathrm{std} \text { bbls }\end{array}$

The Original Gas In Place shown from the EOS studies and the P/Z versus Cumulative Gas Production Reservoir Material Balance was 8.056 BCF. This compared well with the 8.054 BCF from the geologic characterization in the reservoir model.

Pressure matching was the first step in the larger general history matching phase of the study. This was accomplished by looking at the pressure match on the only production well. Later there was an observation well that was used for pressure matching. Since there were very few shut in periods over the life of the production a couple of discrete points in time were used as for a reference comparison. Matching the production and composition of the reservoir fluids were secondary production output variables that were also evaluated.

The loan production well QE2 was used in the pressure matching phase because there were enough shut in periods that allowed comparison and evaluation of how well the matching scenario. As can be seen in Figure $\mathbf{4 . 4 2}$ the History Match Actual vs. Field Data pressures. 


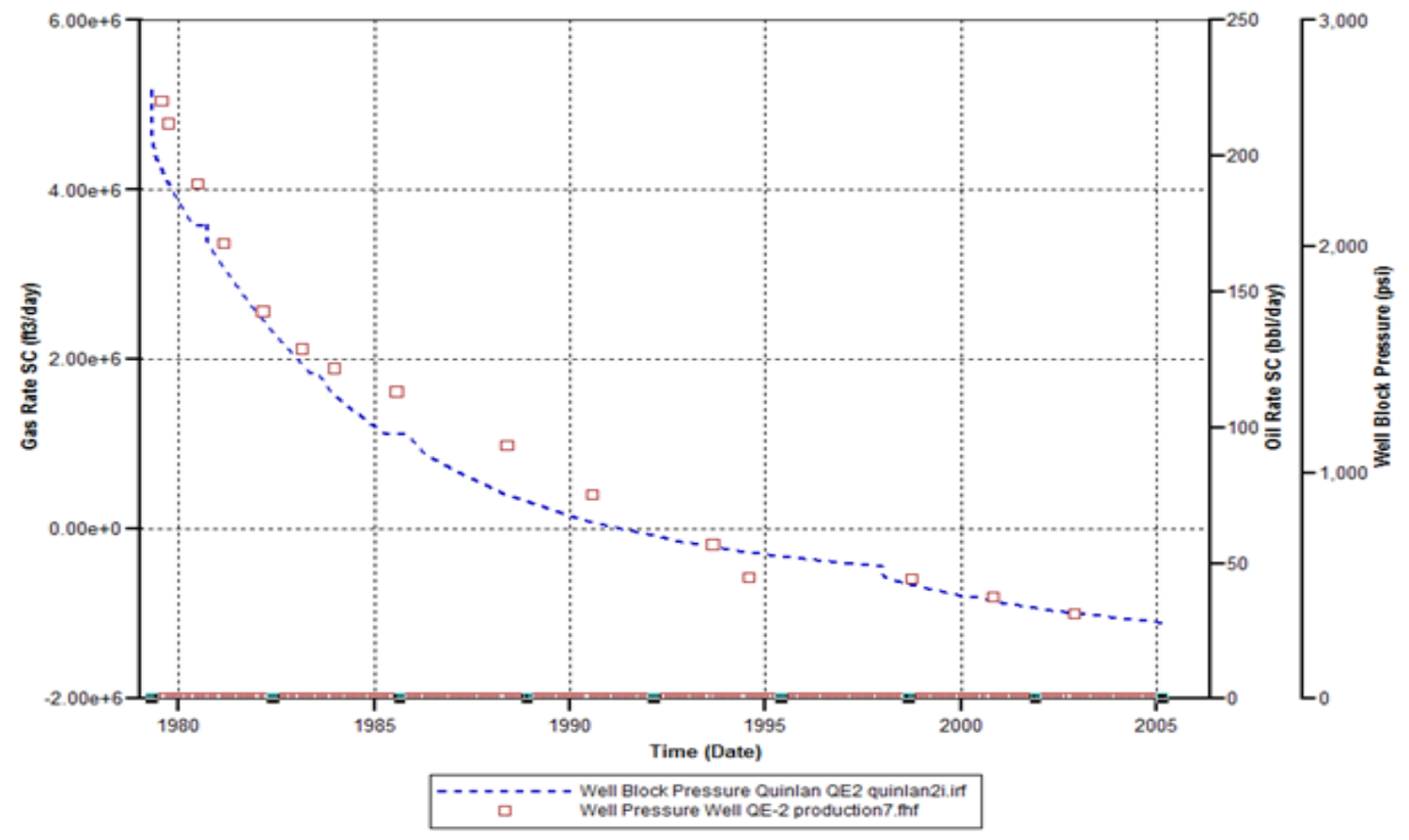

Figure 4.42 History Match Actual vs. Field Data pressures

Pressure Volume Analysis using the Reservoir model and the fluid composition of Case 33 with the WinProp EOS provided a fairly good match with field data. Figure 4.43 shows the comparison of the model predictions for the pressure during the primary production phase to the actual field data collected.

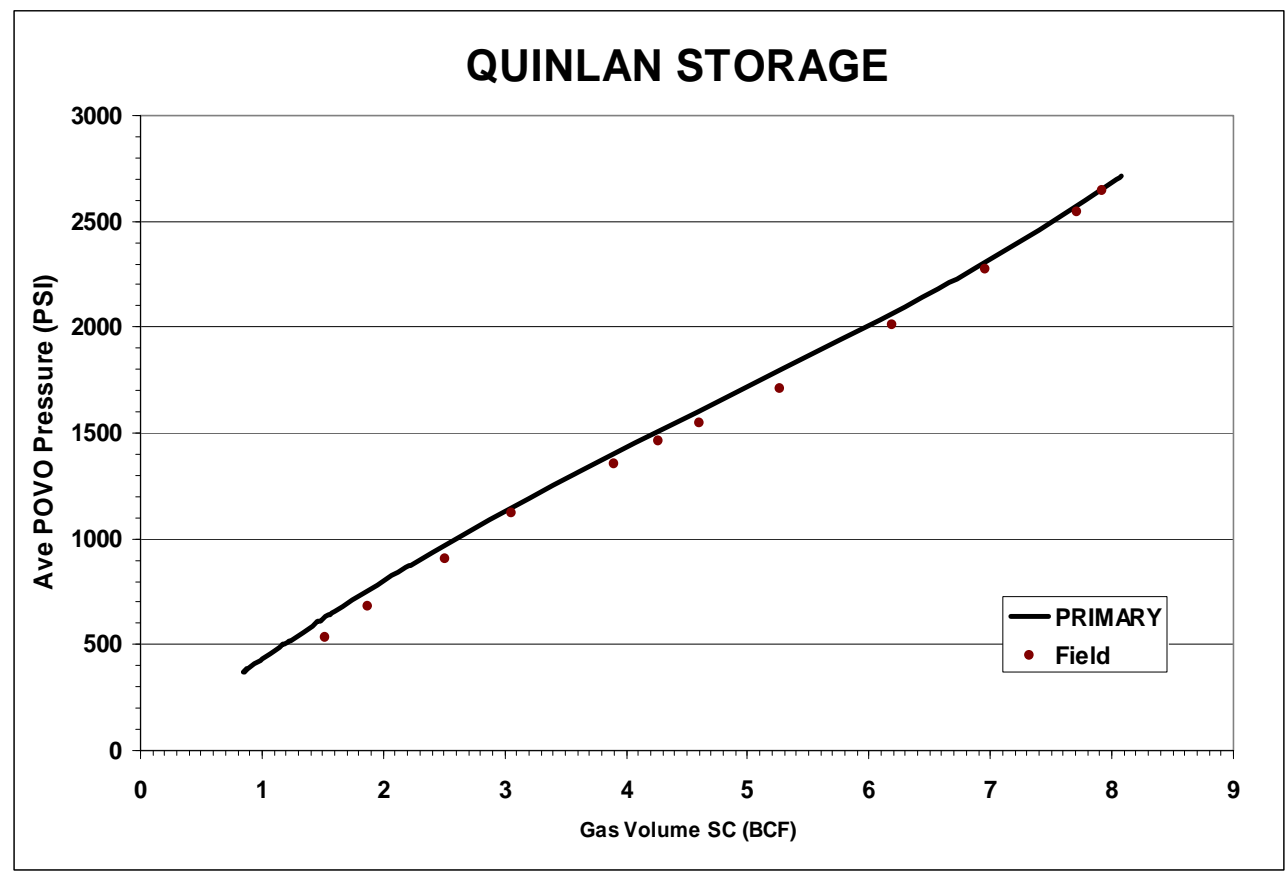

Figure 4.43. Pressure vs. Inv- Actual Field data compared to Model Output 
The graph above depicts the production decline predicted from the reservoir model taking into account the reservoir fluid match believed to exist at the beginning of production at an estimated production schedule. It was reported from the original production data that

The pressure matches fairly well with the actual data until the first major shut in period was observed. The deviation in the observed versus model pressure match was likely caused by the model continuing production during the shut in period. The shut in periods were ignored in the reservoir model because of lack of information and validation.

\section{Simulation of Primary Production}

Developing an accurate model of the reservoir was necessary to predict the degree of fluid mixing and storage performance. Detailed reservoir description obtained from geological data, seismic map, core and well log data were utilized to develop a three (3) dimensional model of the reservoir. There were originally two (2) wells: one production well and one dry hole. The final model description used in this study with the location of the original production well (QE-2) is in Figure 4.35.

One of the outcomes of the production history match was to obtain a representative initial reservoir fluid composition that could be used at the beginning of the primary phase of production. This initial estimated reservoir fluid composition was used to determine if the reservoir was at the dew point or if there existed a significant amount of condensate occupying the pore space.

The ability to match the initial reservoir fluid composition to that of the laboratory analysis plays an important role in the ability to reliably estimate the fluid yields and trends of the fluid streams.

Quantifying the reservoir parameters such as porosity, absolute permeabilities and relative permeabilities throughout the production history is necessary to achieve a representative history match. The reservoir fluid composition and phase distribution and redistribution is critical in achieving a history match that can be relied on for the storage simulations that will be discussed later in this study.

The pore volume of the reservoir was adjusted to match IGIP obtained from the material balance calculations. The model was then produced by assigning a constant wellhead 
(separator) pressure similar to actual field operations. The permeability prediction correlation had to be slightly modified to match the dry gas production during the primary depletion period. The close match between the simulator results and observed production performance provided the confidence in the model to be used for storage predictions. Figure 4.44, 4.45 and 4.46 shown below show the original production history matches;

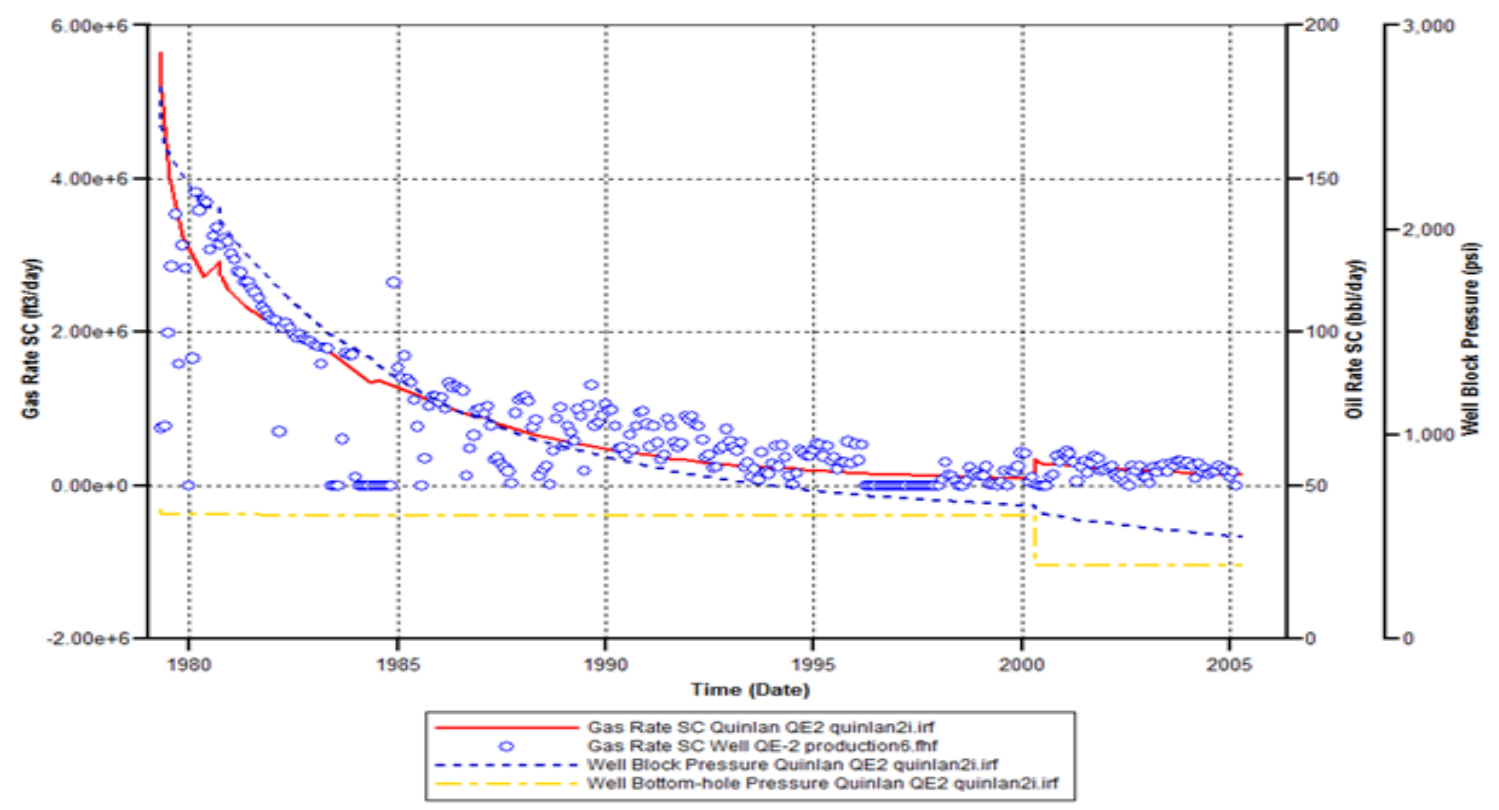

Figure 4.44 History Match Actual vs. Field Gas Rate

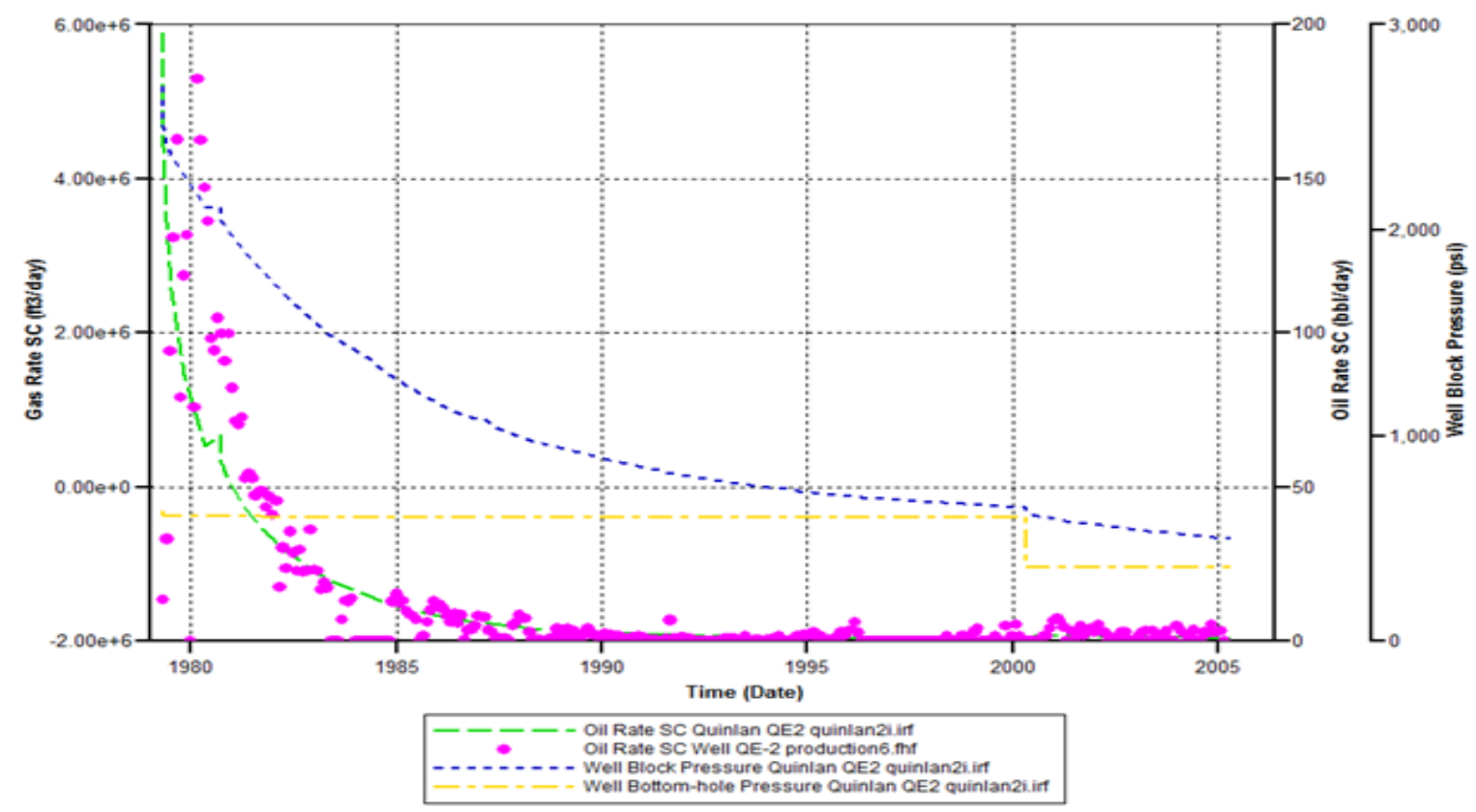

Figure 4.45 History Match Actual vs. Field Oil Rate 


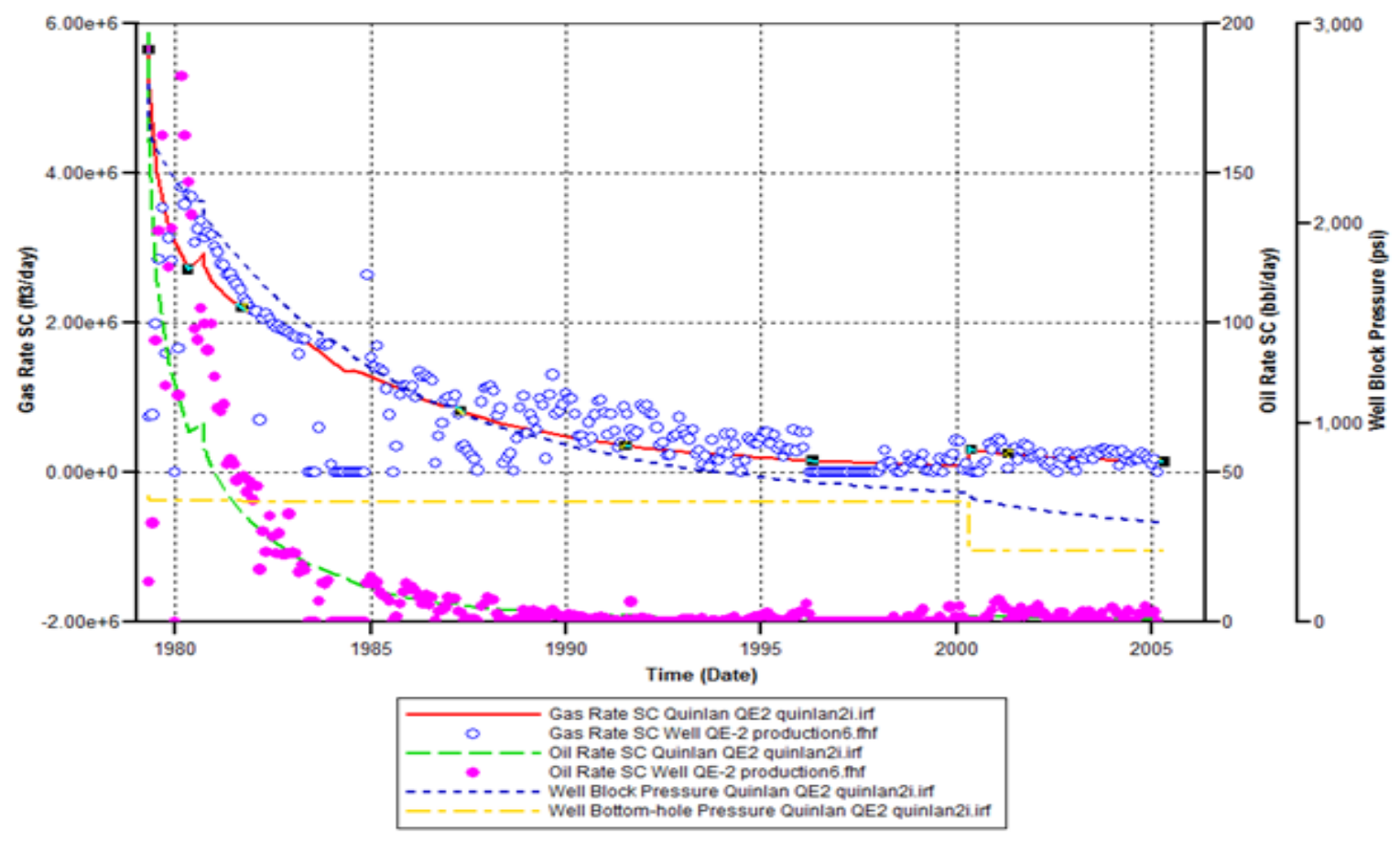

Figure 4.46 History Match Actual vs. Field Combined Oil \& Gas

The history matching phase of the project represented a key step in the development of the reservoir model. As can be seen from the history match plots at the very beginning of the production there was a discrepancy of the time of the start of production. Some of this was due to the delay in getting the well in line and producing and determining on how the condensate liquid production would be handled. As mentioned in previous sections this reservoir was considered a cold condensate reservoir with a temperature of $120 \mathrm{~F}$. Most condensate reservoirs have temperatures in the range of 200 to $250 \mathrm{~F}$ with increased condensation of the liquids and a decreased recovery of the liquids on during the primary phase of production.

In original PVT studies performed in 1979 the Constant Volume Depletion(CVD) study only showed an original STL (Stock Tank Liquids) Ratio of 48.52 bbls/mmscf and a total recovery of $15.77 \mathrm{bbls} / \mathrm{mmscf}$ of the total well stream volume. This represented only $32.5 \%$ of the STL would be produced under the normal depletion schedule suggesting that there would be a significant amount of condensate left in the reservoir at the end of primary production. This was confirmed when compared to the original cumulative Stock Tank Liquid(STL) volumes reported from the operating company. 
The results of the CVD studies indicate that there originally existed 384,000 STB of liquids in the reservoir upon discovery. The production records indicate that 115,000 STB's have been produced to present under primary production. From this it is estimated that there are approximately 250,000 STB's existing in the reservoir at the time of conversion to storage. Based on the actual gas and liquid sales records the CGR started out at approximately 45 $\mathrm{STB} / \mathrm{mmscf}$ and then tapered to a low of $15 \mathrm{STB} / \mathrm{mmscf}$ at the midpoint in the production history and then increasing back to over $30 \mathrm{STB} / \mathrm{mmscf}$ at present. It should also be noted that it is thought that there could have existed some errors in the liquid sales specifically the loss of liquids at the surface (propane, etc.) that occurred during the production phase. The following graph in Figure 4.47 shows the Condensate Gas Ratio (CGR) during the primary production and pressure reduction due to depletion and one versus time. The retrograde phenomena can be distinctively seen by the decline in the CGR recovery where the liquids are dropping out in the reservoir during the primary production. The graph in Figure $\mathbf{4 . 4 8}$ shows this with respect to time that was dependent on the production time schedule.

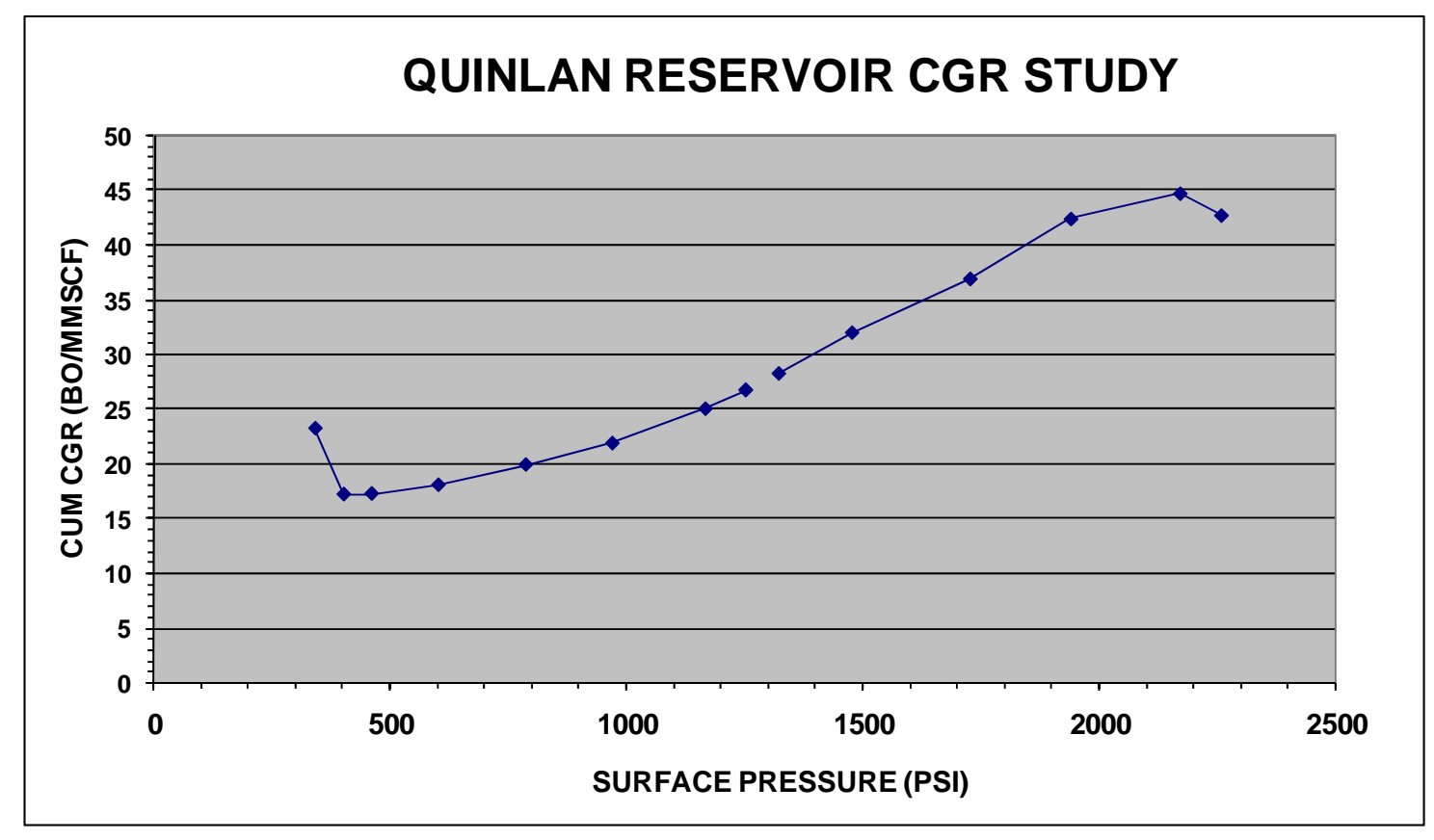

Figure 4.47 Cumulative CGR (BO/MMSCF) vs. Pressure 


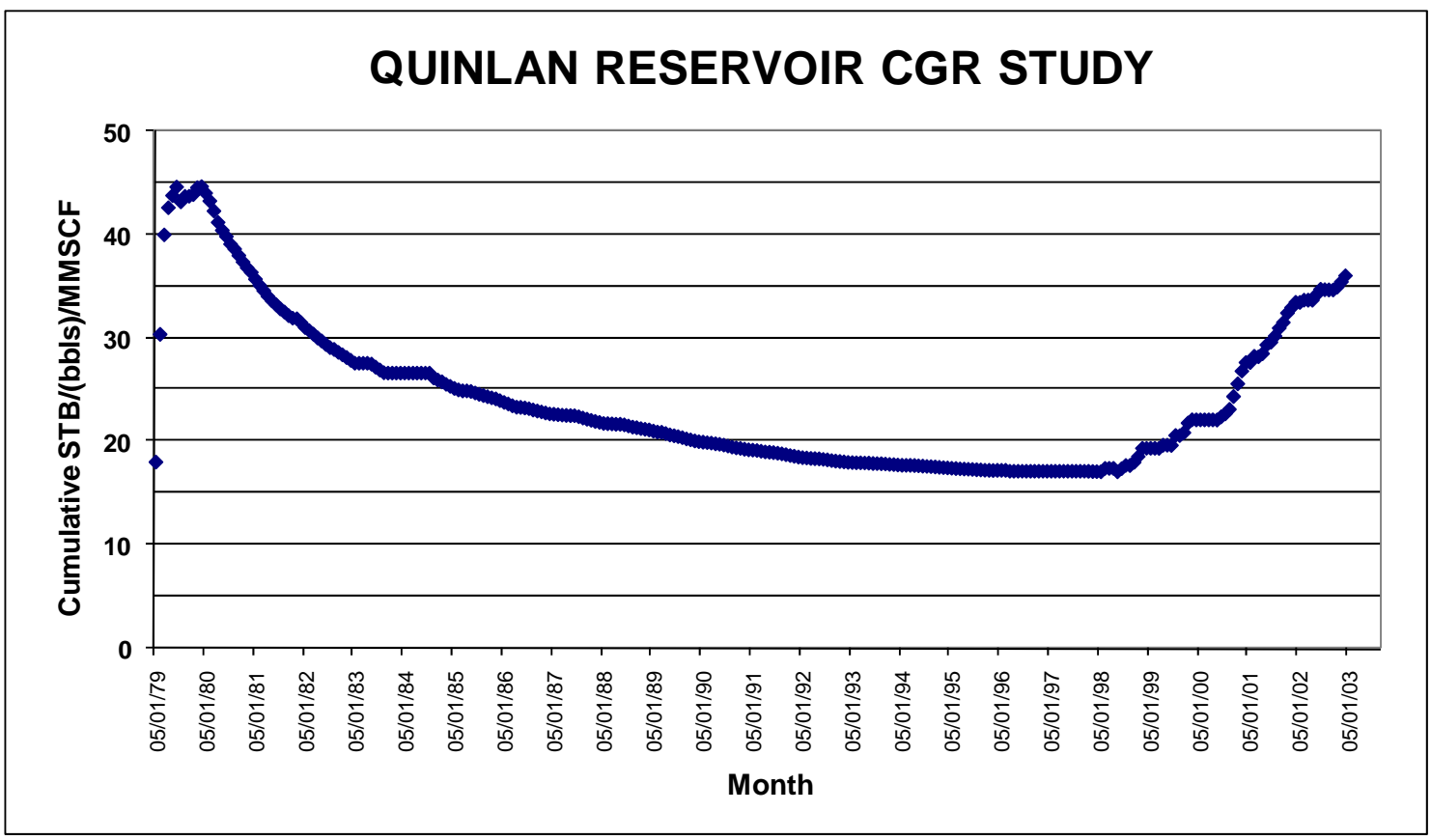

Figure 4.48 Cumulative STB (bbls/MMSCF) vs. Time

\section{COMPOSITIONAL RESERVOIR SIMULATOR COMPARISON}

The second part of the results analysis was to use the reservoir simulation software to study the effects of mixing on condensate recoveries. The reservoir simulation software used in this study was GEM developed by the Computer Modeling Group (CMG). GEM is a robust, general equation-of-state (EOS) based compositional simulator that can model any type of reservoir where the fluid composition and their interactions are essential to the recovery process. GEM can effectively model with complicated phase behavior interactions such as gas condensate storage reservoir and provides extensive well management options and varying designs on surface facilities. A key component of GEM is WinProp multi component phase behavior model that can be used to create the complete PVT data for use in the reservoir simulator.

In our previous investigations an extended compositional analysis for the original reservoir fluid was estimated by obtaining a close match with laboratory measured retrograde dewpoint pressure. A PR-EOS phase behavior package (Aminian 1983) was utilized to evaluate the 
dewpoint pressure for various extended compositional analyses. The matched extended compositional analysis and was then used as the input for PR-EOS phase behavior package to simulate the laboratory study. The results of simulation studies provided reasonable agreements with the laboratory measurements. The measured reservoir pressures and separator pressures were then used in PR-EOS phase behavior package and WinProp to simulate cumulative wet gas production and liquid yield at various pressures. The predicted results were again in reasonable agreement with the field performance. Subsequently, the available pressure and wet gas production data were utilized to perform a material balance study to determine the Initial Gas-in-Place (IGIP). Two sets of 2-phase deviation factors including the predicted values by the PR-EOS and laboratory measured the values were used in the material balance study. Figure 4.49 compares the predicted liquid yields by the EOS model and the simulator for the primary production condensate yield field data. The results clearly indicate that the predictions by the reservoir simulator are superior to EOS predictions. The compositional reservoir simulator accounts for rock-fluid interaction by incorporating the geologic description for the reservoir properties. The EOS tank model is not capable of taking into consideration the reservoir properties.

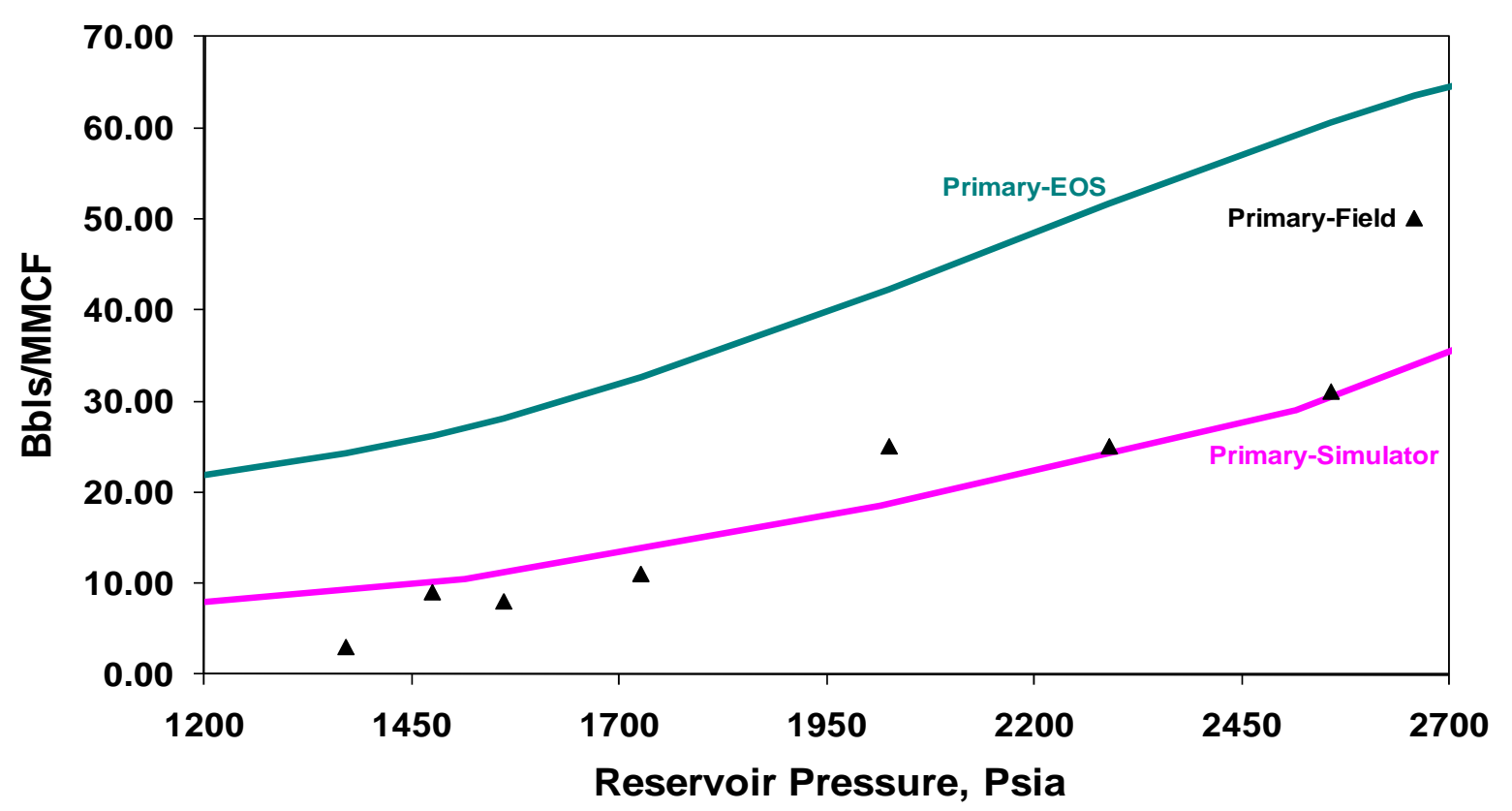

Figure 4.49. Condensate Yield during Primary Depletion 


\section{CHAPTER 5 STORAGE PERFORMANCE AND PREDICTION STUDY RESULTS}

The following chapter summarizes the storage performance and prediction results obtained from the calibrated compositional reservoir simulator. The wellbore configurations, reservoir and fluid property and storage operational scenarios were studied.

The steps to this section are broken three (3) sections:

1. Simulation of Storage Performance with Vertical versus Horizontal Wells

2. Reservoir and Fluid Properties Evaluation

3. Storage Operational Design Evaluation

\section{Simulation of Storage Performance with Vertical Wells}

The model developed in the previous section was utilized to predict the performance of the reservoir during storage operations. The restart option was utilized to initiate the simulation at the conclusion of the primary depletion. Lean pipeline gas was injected into the reservoir utilizing six (6) new vertical injection/withdrawal (I/W) wells. The two existing wells in the reservoir were used as observation wells. Figure $\mathbf{5 . 1}$ is the 3-dimensional grid map of the reservoir model with original and new vertical well locations.

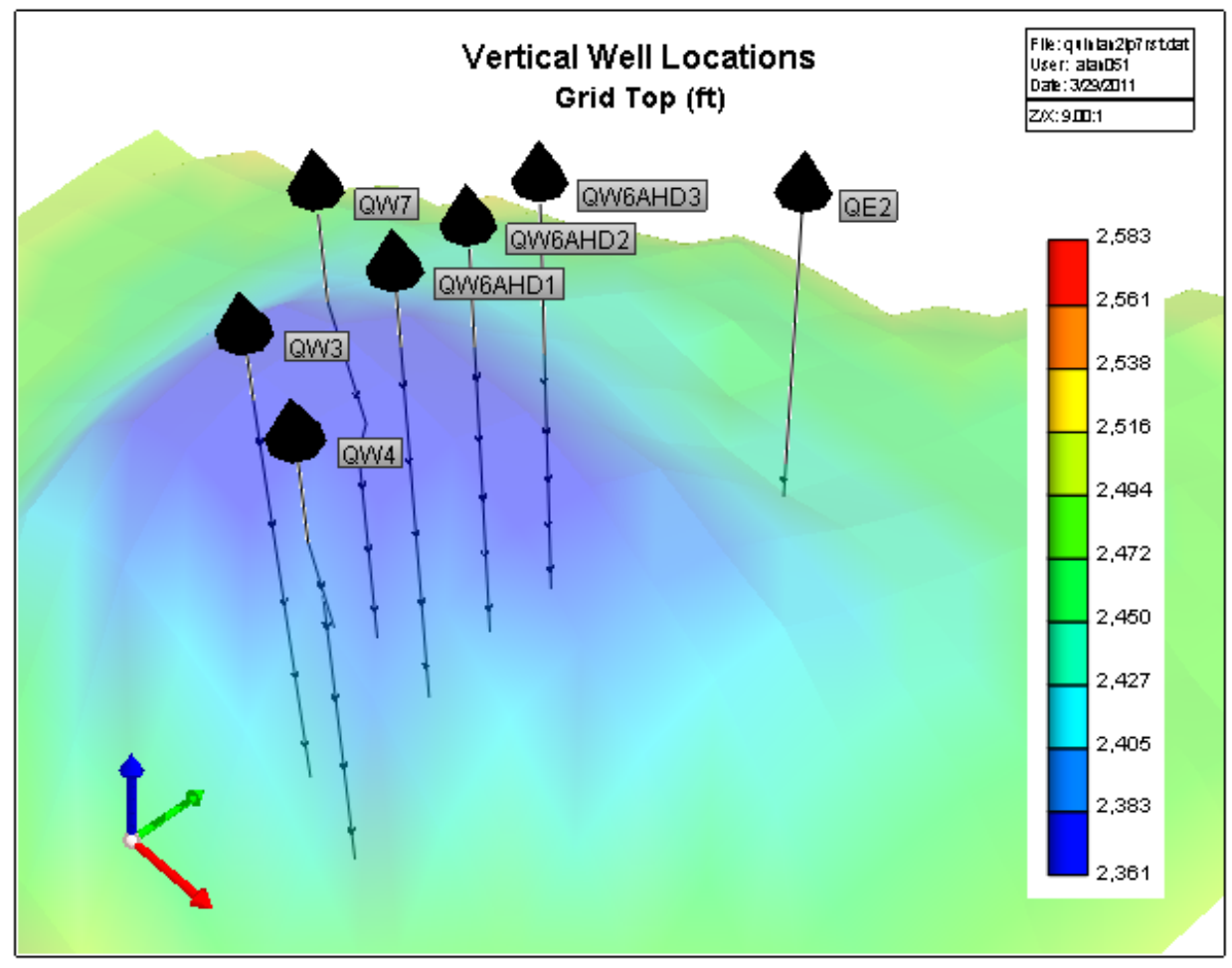

Figure 5.1. 3-DGrid Map of the Reservoir with Vertical I/W Wells 


\section{Simulation of Storage Performance with Horizontal Wells}

The model developed for primary performance simulation was utilized to predict the performance of the reservoir during storage operation. The restart option was utilized to initiate the simulation at the conclusion of the primary depletion. Pipeline gas was injected into the reservoir utilizing 4 new lateral/horizontal injection/withdrawal (I/W) wells. The lateral/horizontal wells were considered because of the reservoir's limited surface access. The two existing vertical wells in the reservoir were used as observation wells. Figure $\mathbf{5 . 2}$ shows the 3dimensional grid map of the reservoir model with original and new horizontal well locations.

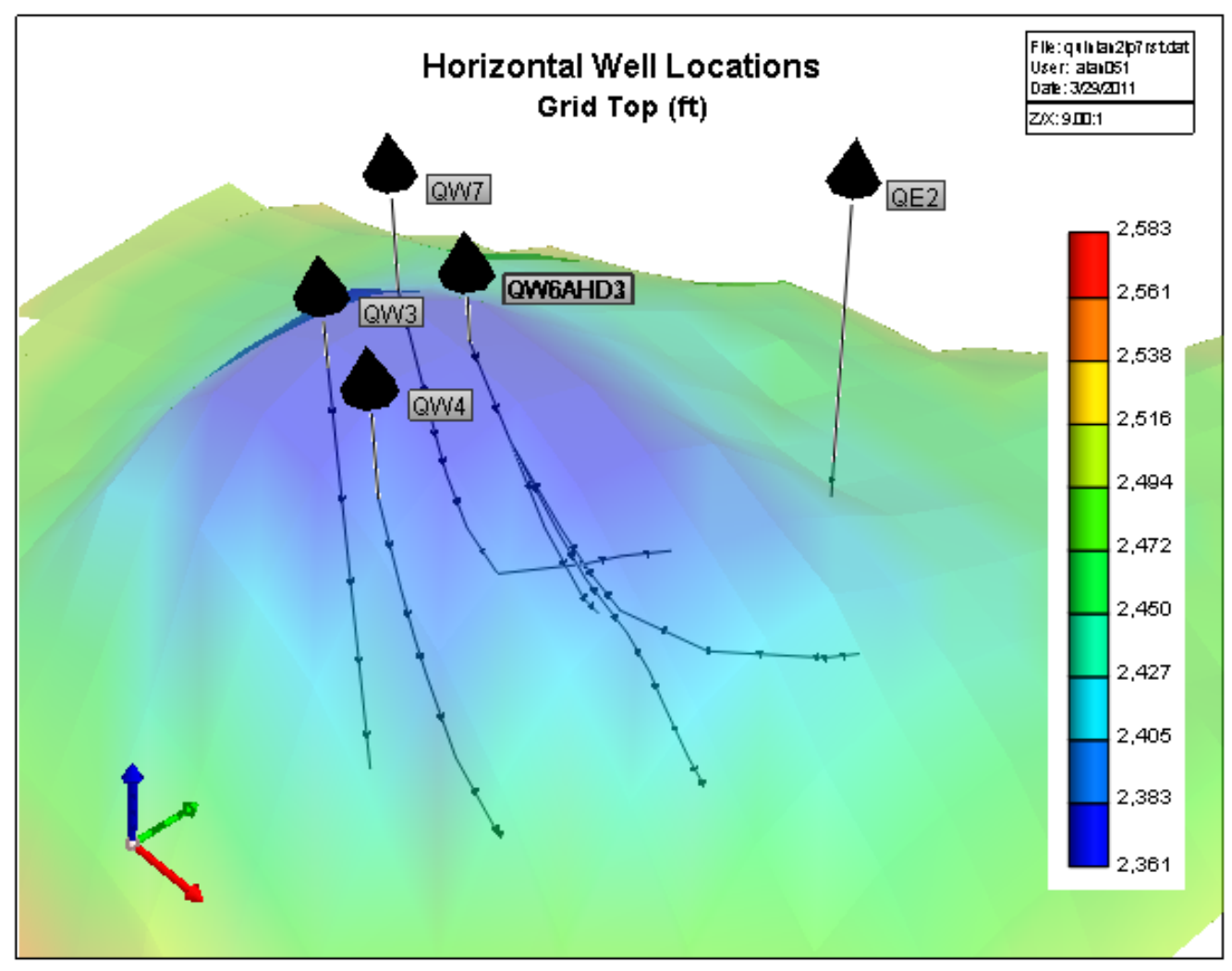

Figure 5.2. 3-D Grid Map of the Reservoir with Horizontal I/W Wells 
The same injection and withdrawal scheme was implemented for both the Vertical and Horizontal well cases. In addition, a constant pressure withdrawal case was also simulated.

Figures $\mathbf{5 . 3}$ and $\mathbf{5 . 4}$ summarize the results of simulation study.

\section{Condensate Rate}

Horizontal vs. Vertical Wells

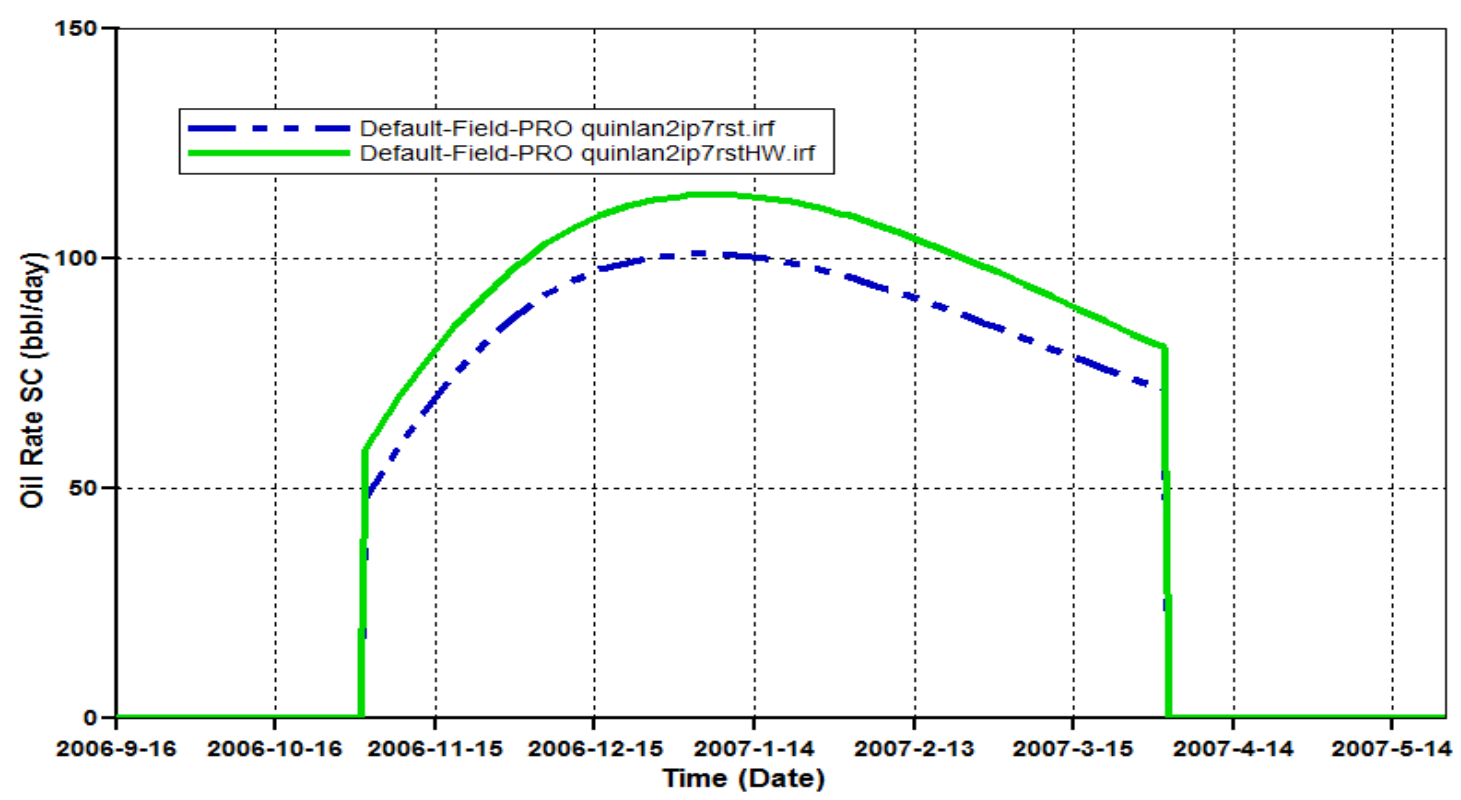

Figure 5.3. Predicted Liquid Production Rates during Storage Operations with Horizontal I/W Wells

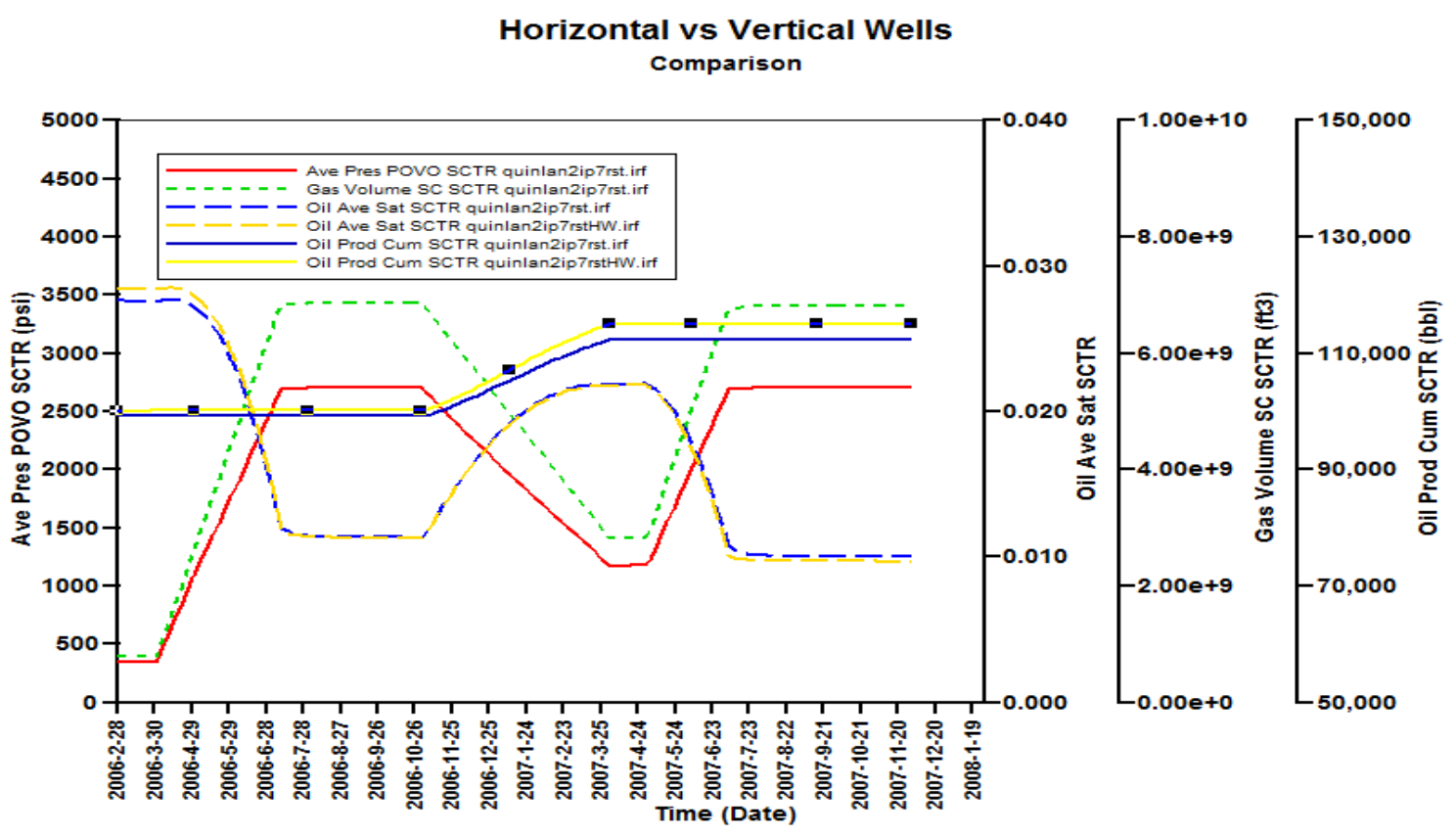

Figure 5.4. Results of Storage Operations Simulation Study with Horizontal/Lateral I/W Wells 
Table 5.1 shows that the Horizontal wells were more effective in mixing with the wet residue and revaporizing the condensate for recovery at the surface during storage operations. Approximately 1800 bbls or $16 \%$ more additional condensate was recovered with the Horizontal wells than would have been with the Vertical wells. The oil saturation or condensate saturation shows that with the Horizontal well management solution the oil saturation was reduced $4.2 \%$ more than with the Vertical well solution indicating that the Horizontal wells decreased the overall condensate in the field.

\begin{tabular}{|c|c|c|c|}
\hline & \multicolumn{3}{|c|}{ Vertical Wells } \\
\hline & $04 / 01 / 06$ & $12 / 01 / 07$ & Diff \\
\hline Ave Pres POVO SCTR & 350.0 & 2704.3 & 2354.2 \\
\hline Oil Ave Sat SCTR & 0.0276 & 0.0100 & 0.0176 \\
\hline Gas Vol MMSCF SCTR & 796 & 6813 & 6017 \\
\hline Oil Prod Cum SCTR & 99162 & 112335 & 13172 \\
\hline
\end{tabular}

\begin{tabular}{|c|c|c|c|}
\hline \multicolumn{3}{|c|}{ Horizontal wells } & \multirow{2}{*}{$\begin{array}{c}\text { HW vs WW } \\
\text { Diff }\end{array}$} \\
\hline $04 / 01 / 06$ & $12 / 01 / 07$ & Diff & \\
\hline 368.1 & 2773.0 & 2404.8 & 50.6 \\
\hline 0.0284 & 0.0097 & 0.0187 & 0.0012 \\
\hline 840 & 6970 & 6130 & 113 \\
\hline 100127 & 115088 & 14961 & 1788.6 \\
\hline
\end{tabular}

Table 5.1. Vertical vs. Horizontal Wells Liquid Recovery Comparison

In Figure $\mathbf{5 . 3}$ it can be seen that initially the Oil rate begins at approximately 50 bbls/d and increases to a maximum of over $100 \mathrm{bbls} / \mathrm{d}$ then near the end of the withdrawal cycle it decreases to $75 \mathrm{bbls} / \mathrm{d}$. The Oil rate from the pool on withdrawal has been shown to be directly affected by the gas rate. The higher gas rate from the pool causes an increased pressure drawdown in the reservoir causing the reservoir to act as a separator trapping the liquids.

The primary simulation results indicate that liquid production and condensate yield are influenced by the reservoir characteristics beyond those that can be predicted by phase behavior simulation. The results of storage simulation with vertical and horizontal wells provide very similar results. This is mainly contributed to high permeability of the reservoir. The constant pressure withdrawal case also provided similar results. However, the withdrawal period was much shorter. This caused slightly lower liquid recovery. The simulation study results however clearly indicate that complete mixing among injected pipeline gas and residue gas and liquid is not achieved. To illustrate this fact, the predicted liquid yields for the first withdrawal cycle for different scenarios are compared in Figure 5.5. 
The EOS prediction are static and assume full mixing while simulator are dynamic and more accurately account for mixing and impact of reservoir characteristics. It can be further noticed that the horizontal wells provided for a slightly better mixing than vertical wells. Detail inspection of simulation results indicated that the liquid saturation and pressure are not uniform throughout the reservoir at the end of primary period.

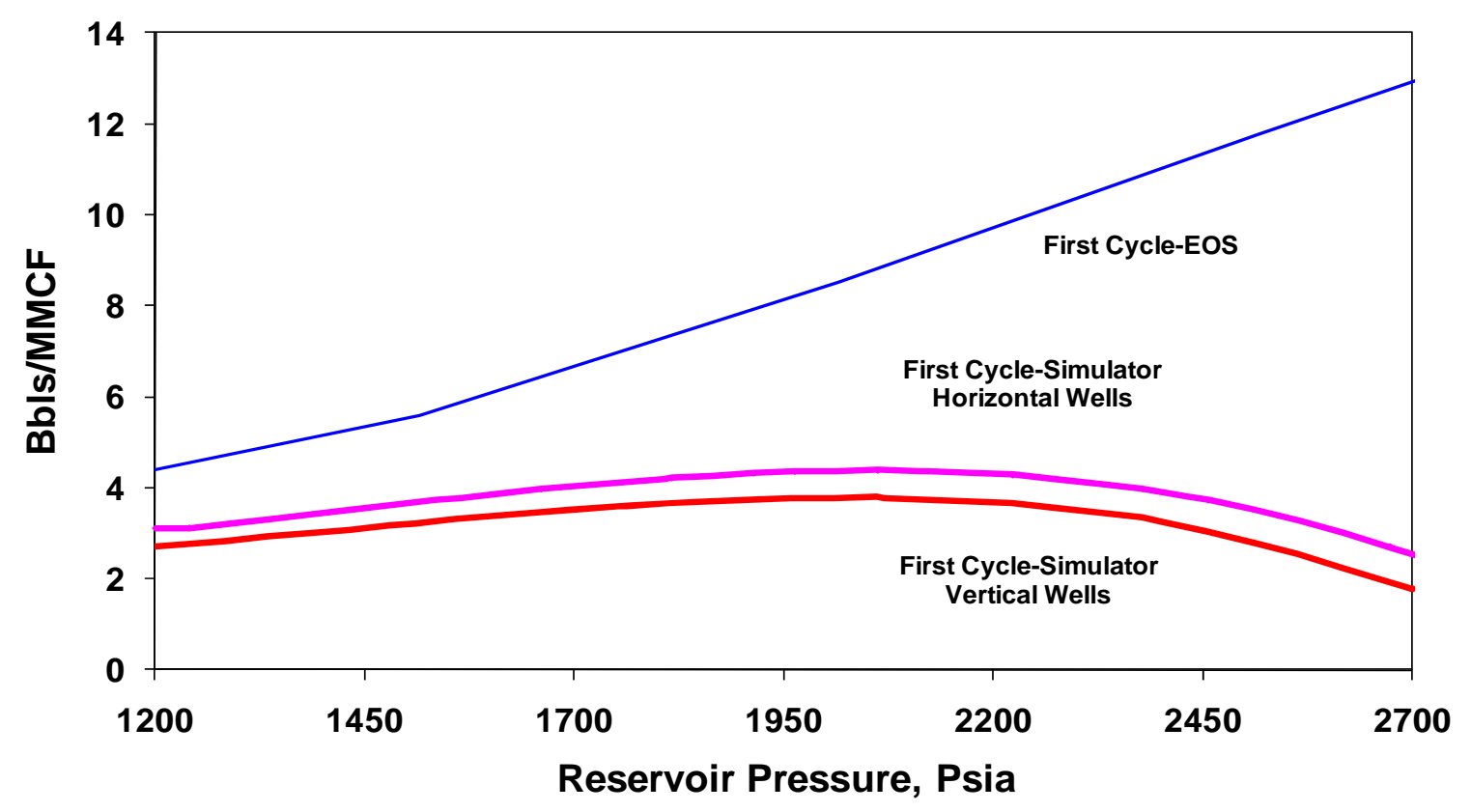

Figure 5.5.: Comparison of Liquid Yields during Storage Operations

The I/W well configuration is such that the gas is injected on top of the structure. This causes the liquid saturation to drop significantly around the injection wells with increased pressure and the contact with dry gas. The liquid saturation in the lower part of the structure away from the injection wells however remains almost unchanged. Therefore, the gas that is injected later during injection cycle does not come into contact with significant residue liquid. This will result in limited enrichment of pipeline gas with native fluids late during injection cycle. The gas injected late will be the first gas that will be withdrawn during withdrawal cycle. It is interesting to note that liquid yields predicted by the simulator reflect this phenomenon. They are initially low and then reach a maximum before they decline again. Initially they are low due to incomplete mixing and final decline is caused by pressure depletion which results in retrograde condensation in the reservoir and entrapment of liquid by the porous media similar to primary depletion behavior but to a lesser extent. The incomplete mixing has both positive and negative impact on the 
operation of storage field. The low liquid yield caused be incomplete or full mixing, reduces the requirement for liquid handling and surface facility design. However, the heavier hydrocarbons remain in the reservoir for a longer time and the liquid production is extended to a higher number of storage cycles.

Another interesting observation in this study was that during injection cycle the reservoir pressure reaches discovery pressure at a gas content nearly one BCF less than original gas content. In Figure $\mathbf{5 . 6}$ below are the model results showing first injection and withdrawal cycle along with the second injection cycle for the pressure versus content. Two factors account for this inconsistency in gas content. First, some liquid remain in the reservoir (approximately 1\% of pore volume) even when the reservoir pressure has reached the initial pressure. Second and more importantly, the injected lean pipeline gas has much lower gravity that the original fluid and it compresses less than original high gravity reservoir gas. This phenomenon will occur in any gas condensate depleted reservoir that is converted to storage. However, the impact of the compressibility change is not readily observed because the compositional difference between injected gas and native gas are not as drastic as the case here. Secondly, many gas reservoirs are converted to storage with much higher native fluid content and as a result the compressibility changes are small. In this reservoir, nearly 88 percent of native fluid had to be replaced with injected dry pipeline gas causing about 12 percent reduction in gas content. It must however be noted that this is economically advantageous. Since the significant portion of injected gas was designed to be used as the base gas, this reduction in gas content directly reduces the base gas requirements. The reservoir has sufficiently high deliverability that this reduction in gas content does not influence the deliverability of the reservoir.

The reduction in base gas as a result of the gas composition is further complemented because the reef reservoir is so prolific and allows for almost no pressure coning due to storage cycling. Typically in poorer reservoirs the pressure in observation wells on withdrawal show much higher and much lower on injection than pressures on injection/withdrawal wells. The horizontal well management plan selected for this reservoir further helps in decreasing pressure drawdown resulting in improved condensate recovery, less base gas requirement and reduced pressure coning. These factors make the carbonate storage reservoir act much like salt cavern storage. 


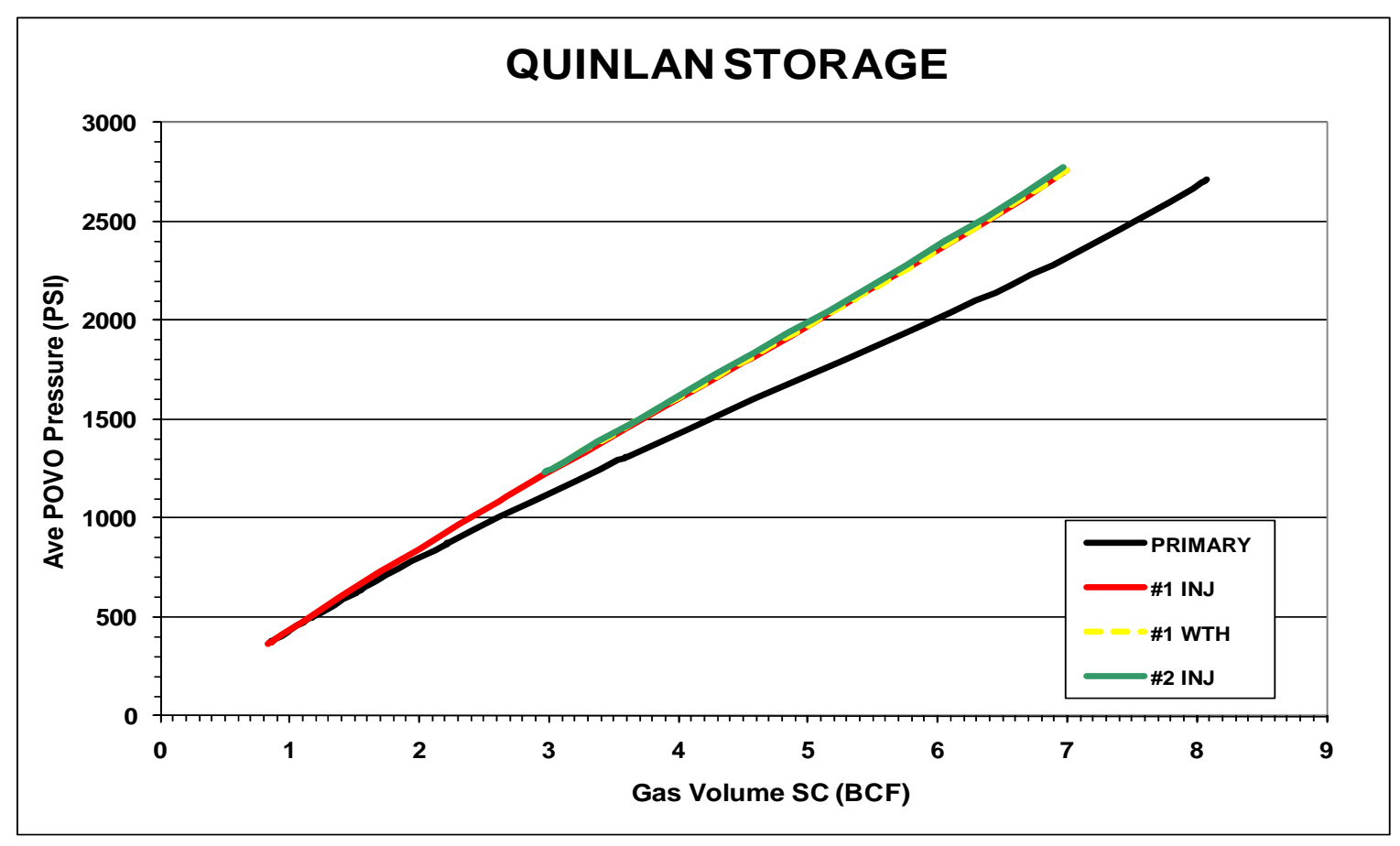

Figure 5.6. Reservoir Pressure as a Function of Gas-in-Place

As a result of the decision to develop the reservoir for storage operations based on the EOS and Compositional models several questions were raised during development and after a couple of complete storage cycles. The importance of the models ability to predict condensate recovery and reservoir performance have been vital in answering questions such as, design of surface facilities, prediction of variable peaking and base load storage operations on condensate. When a storage pool is developed usually the pool is tested to see if certificated levels of deliverability can be met as well as the ability to reach the maximum designed storage capacity. The model has been used to study the problems associated with condensate recovery in storage operations. Manpower scheduling, adequate condensate storage, quality of condensate, safe operations are just some of the problems studied. As with most storage reservoirs in the Northeast U.S. the weather can become a factor in operations. The model was initially used to determine how much on-site what condensate storage was necessary to handle the logistics of hauling the condensate product to the purchaser along with the understanding of weather constraints. Another area the model was used for was in the design of the surface facilities relating to flaring options or implementation of flash gas recovery units. This became evident in the air permitting requirement for VOC's and other pollutants. Different modes of operation have been studied to see the impact on condensate recovery like the earlier studies 
done by Katz et al (1981) The sale of the condensate becomes important financially because accurate predictions of liquid condensate rates and volumes are required to obtain and keep the best contracts.

This part of the study was broken down into the following two (2) sections:

\section{1) Reservoir and Fluid Properties Evaluation \\ 2) Storage Operational Design Evaluation}

The reservoir and fluid properties evaluation section is devoted to studying the effects of reservoir properties like permeability variations, near well bore skin effects and how these impact mixing and subsequent condensate recovery.

The storage operational design evaluation section mainly focuses on injection /withdrawal patterns (i.e high rates, etc.) and how resident time impacts diffusivity and mixing. Also how to improve surface facilities performance and optimization.

\section{Reservoir and Fluid Properties Evaluation}

In Aminian et al (SPE 111193 2007) the compositional reservoir simulator was used to predict the condensate recovery for the first storage cycle. These results are shown in Figure $\mathbf{5 . 7}$ where the model predicted condensate yields are compared to the actual field measured volumes.

The model results indicated that more lean storage gas occupies the pore spaces near the wellbore of the injection and withdrawal wells than in the perimeter areas away from the wells. When the withdrawal cycle begins the lean unmixed gas near the I/W wells will first be produced(FIFO). This causes the initial liquid yields to be low because of incomplete mixing. As the production continues, the gases away from the well will be produced and as result the liquid yields will increase. However by the time the heavier gas reaches the wellbore, the reduction in the reservoir pressure results in retrograde condensation in the reservoir and entrapment of liquid by the porous media similar to primary depletion behavior but to a lesser extent. The liquid yield at the surface increases slowly and subsequently declines upon additional pressure depletion. 


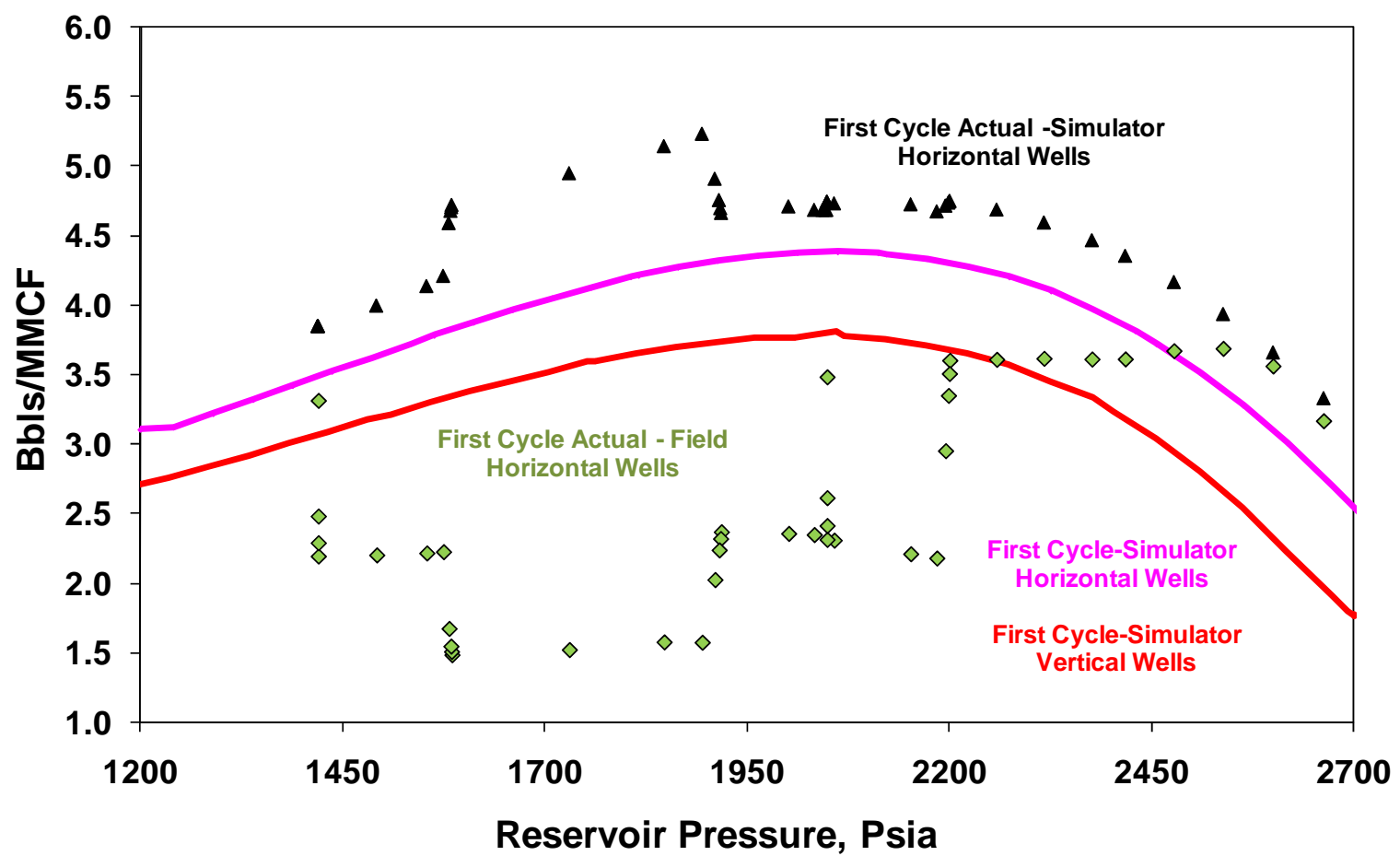

Figure 5.7. Comparison of the Liquid Yields during First Storage Cycle

Further investigation of Figure $\mathbf{5 . 7}$ shows that early during the withdrawal cycle the liquid yields compared closely with the model predictions. The liquid yields were significantly lower during the rest of the cycle and towards the end of the cycle they began to approach the model predictions. Initially the withdrawal rate was started at $30 \mathrm{mmscfd}$ and then raised to $50 \mathrm{mmscfd}$ followed by a final maximum rate of 75 mmscfd before finishing up the season at the lower 50 mmscfd. Every time the rate was increased the liquid yield decreased correspondingly and when the rate was reduced at the end of the cycle the liquid yield approached the model predictions. The exact same rate history actually followed during the withdrawal season was used in the model for the predictions. Therefore, it was important to understand the reason for the differences between the model predictions and the field observations. 
The following factors were thought to be possible reasons for sensitivity of the liquid yields to withdrawal rate that are not accounted for in the model:

1. Reservoir fluid compositional analysis

\section{Reservoir heterogeneity}

3. Impact of the wellbore

4. Inaccuracy in liquid measurements

5. Efficiency of surface facilities

As was mentioned earlier, the extended compositional analysis of the original reservoir fluid was not available and was estimated by obtaining reasonable agreements with the laboratory measurements. The model predictions for depletion period indicate that the predicted liquid yields are not significantly different than field results. Further, the impact of original fluid composition on liquid yields would be less significant during storage cycle since more than 90 percent of the original fluid is replaced by the pipeline gas. Additionally, while the exact compositional analysis could have provided more accurate liquid yield predictions, it could not explain the sensitivity of liquid yield to the flow rate.

The mixing of the various fluids in the reservoir is impacted by reservoir heterogeneities. Therefore, it is possible that the higher flow rates can cause a different gas mixture to be produced due to reservoir heterogeneities. To investigate this concept a few simulation runs were conducted by altering reservoir characteristics in different layers. The reservoir is consisted of 5 layers. The results of these runs are illustrated in Figure $\mathbf{5 . 8}$ showing although heterogeneities altered the liquid yield predictions, they could not account for the reason liquid yields were affected by flow rate. 


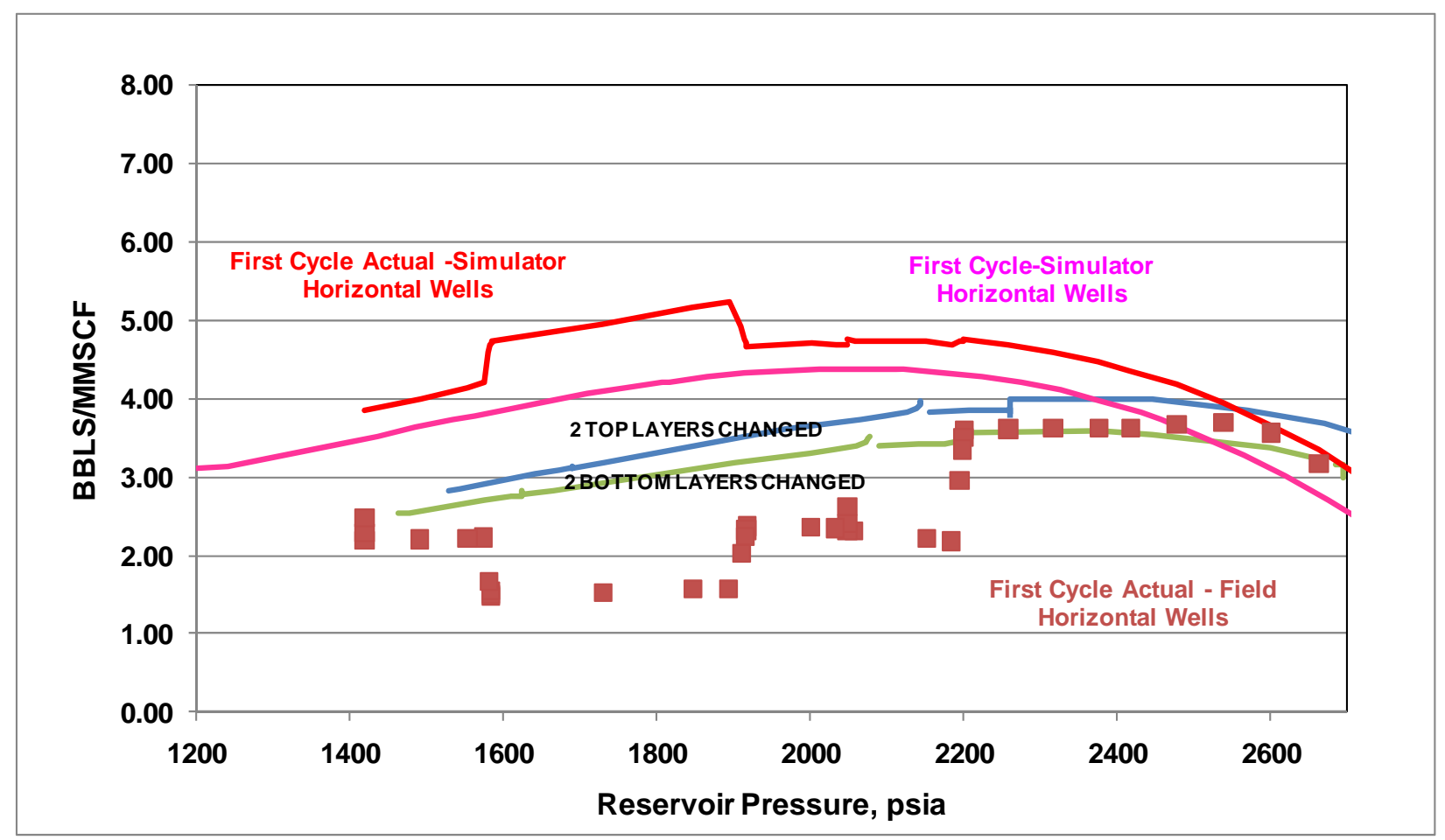

Figure 5.8. Impact of Heterogeneity on the Predicted Liquid Yields during First Storage Cycle

\begin{tabular}{|c|c|c|c|c|}
\hline & $\begin{array}{c}\text { Default-Field- } \\
\text { INJ } \\
\text { Cumulative } \\
\text { Gas SC }\end{array}$ & $\begin{array}{c}\text { Default-Field- } \\
\text { PRO } \\
\text { Cumulative } \\
\text { Gas SC }\end{array}$ & $\begin{array}{c}\text { Cumulative } \\
\text { Oil SC }\end{array}$ & $\begin{array}{c}\text { Ave Pres } \\
\text { POVO SCTR }\end{array}$ \\
\hline Case 1 Perm Formula 3 Top 2 Layers & 24166297600 & 25572409344 & 46364 & 2692 \\
\hline Case 2 Perm Formula 3 Bottom 2 Layers & 24384288768 & 26420262912 & 45073 & 2426 \\
\hline Diff & -217991168 & -847853568 & 1291 & 266 \\
\hline
\end{tabular}

Table 5.2. Comparison of Permeability Reductions in Grid Layers

In Table 5.2 Comparison of Permeability Reductions in Grid Layers show the updated data for the last three (3) storage cycles. The reservoir model allows for global changes to the reservoir properties by assigning formulas to the layers and grid blocks. To simulate the reduction in permeability a permeability formula was developed that would reduce the permeability by $50 \%$ of the original. This formula was applied to the top two layers and the bottom two layers. Figure 5.8 and Table $\mathbf{5 . 2}$ show that reductions in the permeability had less effect on the top two 
layer scenario and more effect on the bottom two layers. In Case1 where the permeability in the top two layers were reduced yielded 1291 bbls more with a higher average pore pressure of 266 psi than the bottom two layer change scenario. The cumulative amount of storage gas injected and produced was less with Case 1 than with Case 2. This was probably due to the top 2 layers is more restrictive around the wellbore preventing flow of gas and enhancing the recovery of oil. When the bottom two layers permeability is reduced the restricted effect of gas moving toward the wellbore at the top of the reservoir is less possibly dropping out more condensate in the reservoir.

The model does not account for pressure drop in the wellbore and pressure drop in the wellbore could result in liquid drop out and accumulation in the wellbore. This is particularly true for horizontal/inclined wells. The pressure drop, and as the consequence the liquid drop out, in the wellbore is flow rate dependent. However, the limited volumes of the wellbores do not allow for significant liquid accumulations. This could provide only a partial explanation for lower liquid recovery at the surface.

The liquid production was estimated based on liquid level collected in the storage tank and as a result was not very accurate. Furthermore, the temperature variation may have contributed to loss of the liquids to vapor phase. However, these inaccuracies do not seem to be rate dependent and as result cannot provide a justification for differences between the predicted and actual liquid yields. The efficiency of the liquid separation facilities at surface could contribute to the loss of liquid to the gas phase. If the separation facilities reach the maximum recovery capability at lower gas rates, the increase in rate will not result in additional liquid recovery and the liquid yield appears to drop off. To investigate this concept, the liquid yields were recalculated based on constant gas rates. Figure 5.9 illustrate the revised liquid yields based on two different constant withdrawal rates. As it can be seen, the revised yields are well within the predicted ranges by the model. It should however be noted that there are no independent evidence available to verify if this phenomenon is occurring in the field. 


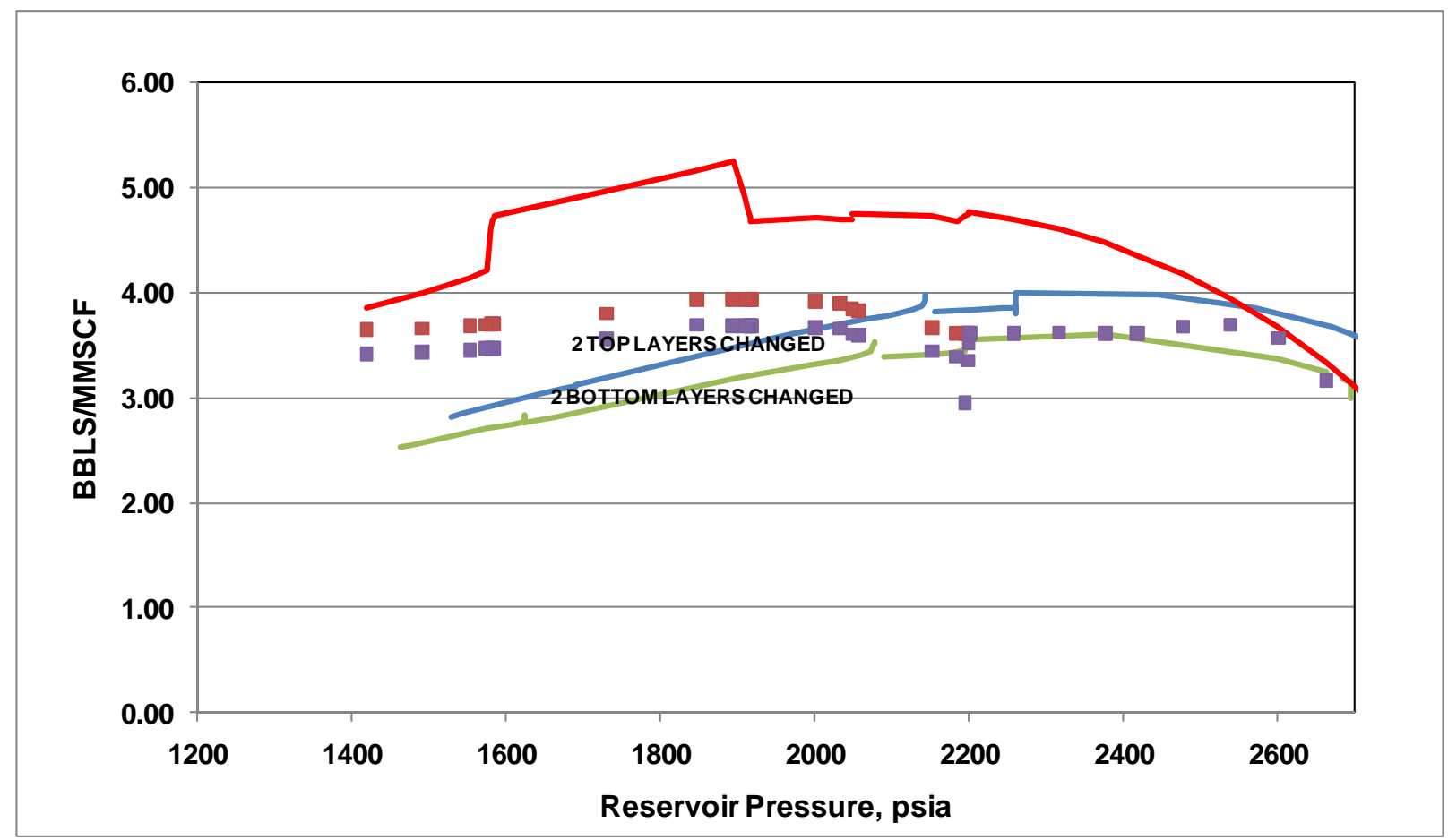

Figure 5.9: Comparison of Model Predictions and Revised Liquid Yields during First Storage Cycle

To validate the feasibility of the reservoir heterogeneities causing yield discrepancies between the actual and model predictions it was proposed to go back to the original history match and make a comparison run with the permeability reduction changes to the top two layers.

\section{Affects of changing the Permeability in the Top Two Layers of the Original History Match}

The changes made to the original reservoir description pertaining to reducing the permeability in the top two(2) layers by $50 \%$ of the original history match model run reduced the recoverable gas from 7.066 BCF to 6.175 BCF. The recoverable oil was not affected as much actually increasing from 90.077 MBBls to 92.367 MBBls. The average pressure in the original model showed 369 psi in the HC pore volume and with the perm changes showed 707 psi. The initial average gas production rate was reduced that affected the total recovery. The model runs can be seen in Figure $\mathbf{5 . 1 0}$ below: 


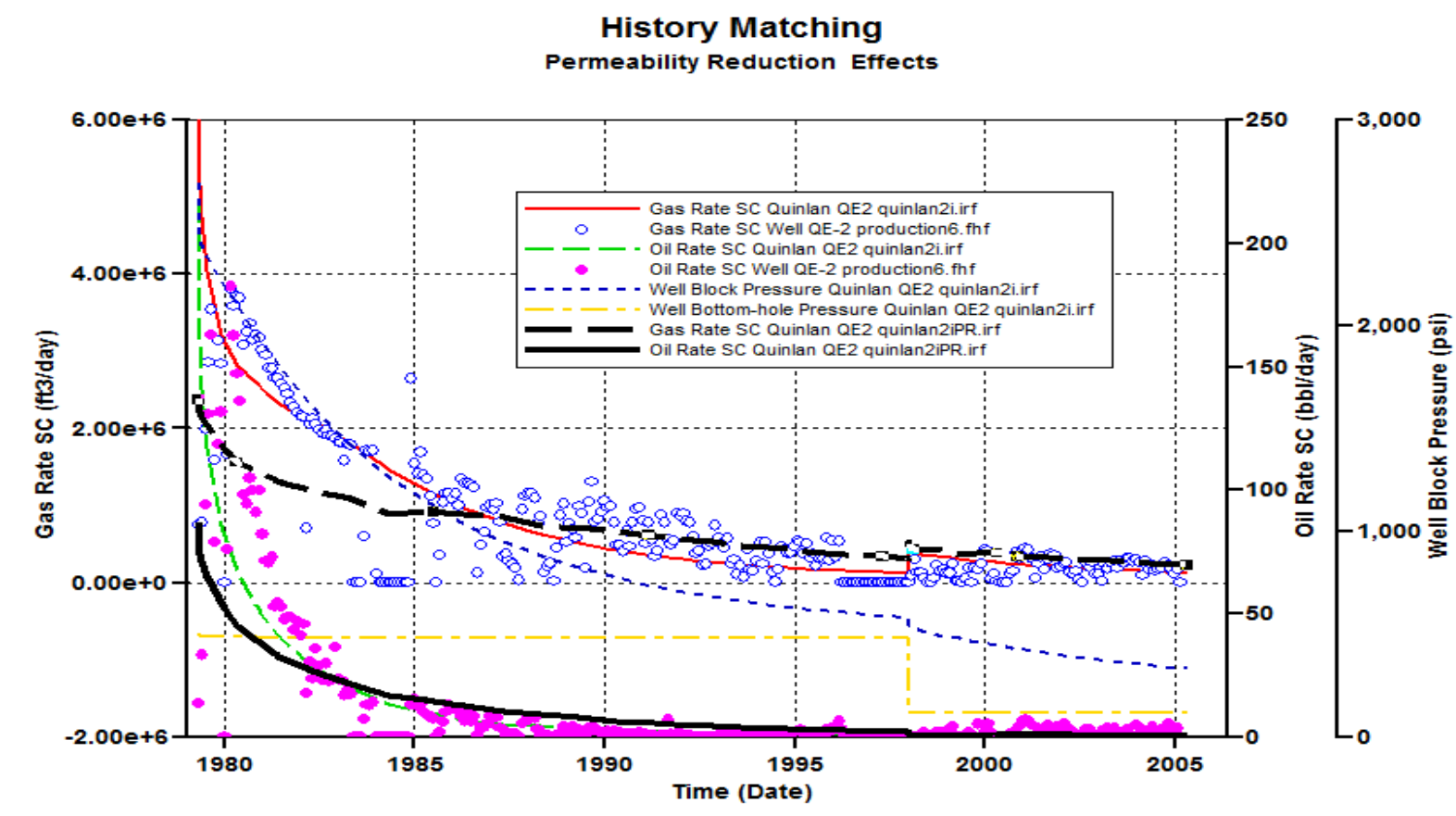

History Match Comparison using 50\% permeabiltiy reduction in Top two Layers

\section{Figure 5.10: Original Production History Match Comparison w/ Perm Reduction in top two layers}

In summary, the analysis of the reservoir description change associated with the change in the Perm Formula that reduced the permeability of the top two (2) layers of the model decreased the gas recovery and increased the oil recovery. One note on why this might have occurred is that the original production well was located in a poor part of the reservoir and was affected by the permeability change more than if the well was in the main part of the reservoir where the storage operations are now being conducted. Comparing these runs shows that reservoir heterogeneities cannot explain the yield discrepancies during the first storage cycle as shown earlier between the actual and model predictions. 


\section{Affect of Primary Production Well Location on Original History Match}

Understanding that the original production well was not drilled in the best part of the storage field and actually just intercepting the edge of the reservoir boundary. It was proposed to move the original production well to a better location and see how the reservoir properties affected the original production history match. Figure $\mathbf{5 . 1 1}$ shows the new well location changed from $16,14,1$ to $14,16,1$ where the porosity increased from $10 \%$ to $14 \%$, the Net Pay increased from 70 to $100 \mathrm{ft}$ and the permeability changed from $3.4 \mathrm{md}$ to $13.4 \mathrm{md}$.

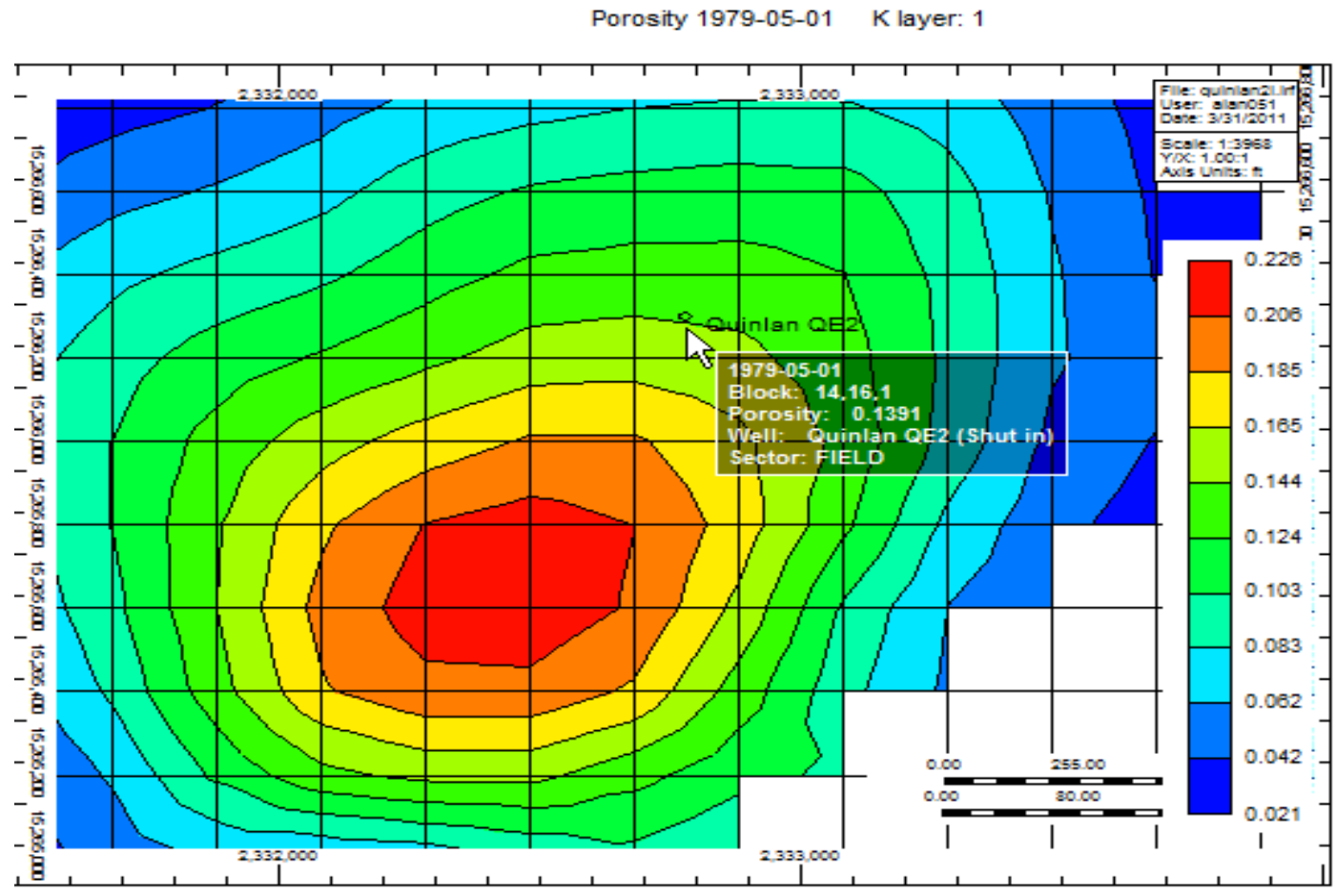

Figure 5.11: Map of Original Production New Well Location change $(14,16,1)$

The model results show that by placing the original production well in a better part of the reservoir the recovery of gas increased from 7.067 BCF to 7.562 BCF and the oil recovery increased from 92367 bbls to 93214 bbls. The average pore volume pressures also decreased from 369 psi to 159 psi because of the production of an additional 495 mmscf of gas. The simulation results are shown below in Table $\mathbf{5 . 3}$ and Figure 5.12: 


\begin{tabular}{|ll|c|c|c|}
\hline \multirow{2}{*}{ FIELD SECTOR-1 } & \multicolumn{2}{|c|}{ QE 2 Location } & \multirow{2}{*}{ Diff } \\
\cline { 2 - 3 } & Orig & Better & \multirow{2}{*}{$\begin{array}{c}\text { Diff } \\
\end{array}$} \\
\hline
\end{tabular}

Pore Volumes

\begin{tabular}{|l|l|r|r|r|}
\hline Total Pore Volume. M rft3 & & 44275 & 44237 & -38.000 \\
\hline HC. Pore Volume M rft3 & & 32876 & 32882 & 6.000 \\
\hline
\end{tabular}

Average Pressures

\begin{tabular}{|l|r|r|r|r|}
\hline Cum Water Influx M STB & & 369.2 & 159 & -210.20 \\
\hline Cum Water Influx M STB & & 369.11 & 158.87 & -210.24 \\
\hline Cum Water Influx M STB & & 369.11 & 158.87 & -210.24 \\
\hline
\end{tabular}

Cumulative Production

\begin{tabular}{|l|r|r|r|r|}
\hline Oil M STB & & 92.4 & 93.2 & 0.8 \\
\hline Gas MM SCF & & 7066.7 & 7561.5 & 494.8 \\
\hline Water M STB & & 0.070 & 0.147 & 0.077 \\
\hline Wet Gas MM SCF Gas & & 289.5 & 320.9 & 31.4 \\
\hline
\end{tabular}

Table 5.3. Comparison of Results w/ Original Production Well Location change History Matching

Well Location Change from $16,14,1$ to $14,16,1$

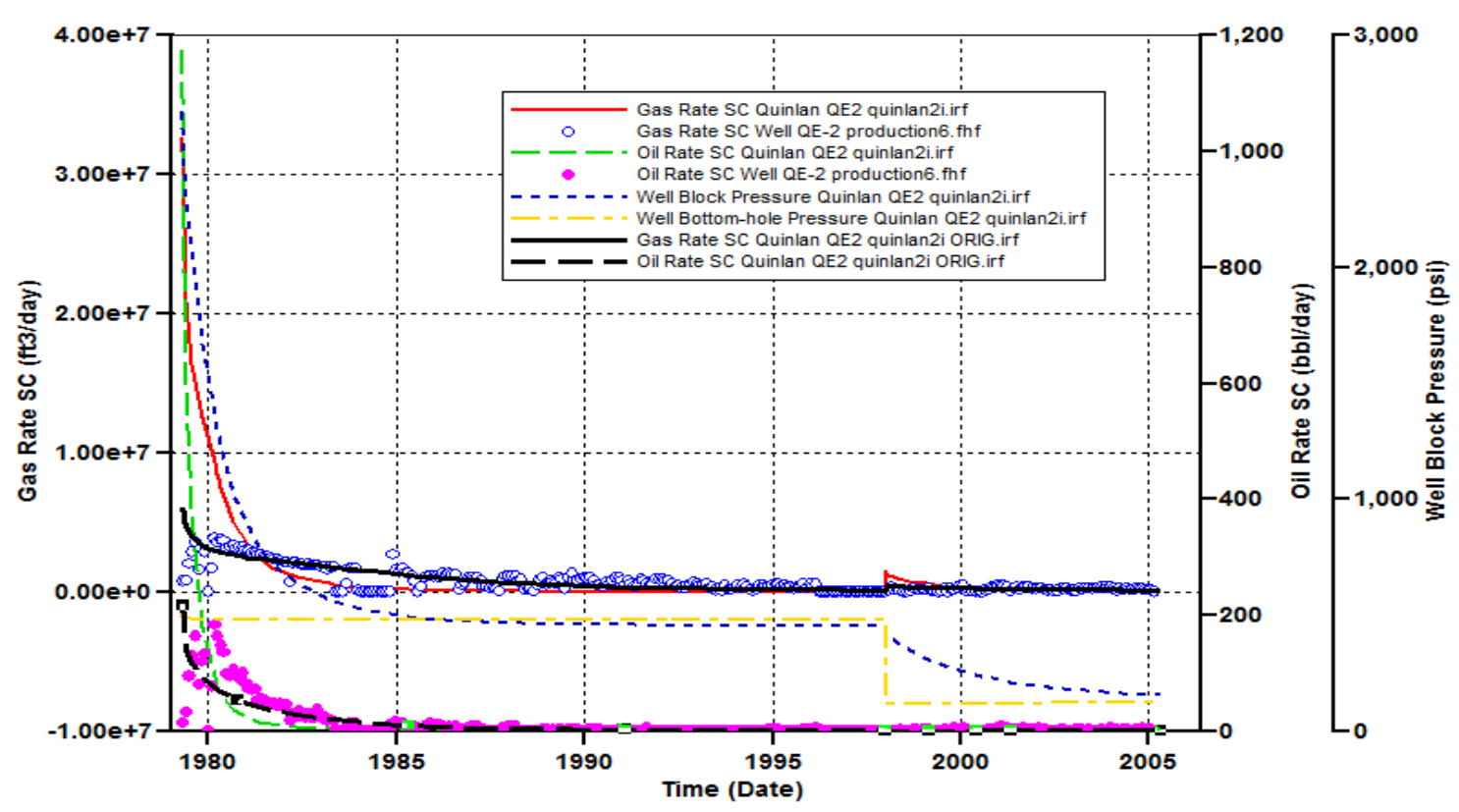

Figure 5.12: Comparison of Results wl Original Production Well Location change 


\section{Evaluation of Wellbore Effects and Condensate Influence on Condensate Yields}

Since there has been much literature written discussing the problems associated with condensate blockage near the wellbore causing deliverability restrictions, this part of the study addressed those important concerns. An evaluation of the wellbore effects on condensate blockage or Non Darcy flow effects was performed to see how these Skin Factors influence condensate yields. Both near wellbore and at the reservoir extents were studied to validate the thoughts that condensate blockage would not affect the deliverability requirements and total capacity estimates.

Many gas condensate reservoirs have liquid accumulation around the wellbore that can significantly reduce deliverability(Firoozabadi et al 2007). At liquid saturations below $5 \%$ in the reservoir well below the point of fluid flow and mobiltiy due to adsorption to the porous media these factors might be of lesser concern.

The following simulation runs were made using the following schedule and well index changes:

1. Change Skin factor to 2 Resulted in no change in relationship

2. Change Skin factor to 20 Resulted in no change in relationship

3. Change Skin factor to 20 , Turb Skin ON Resulted in no change in relationship

4. Change Skin factor to 20 , Turb Skin ON ,Quadratic Flow ON Resulted in no change in relationship

The notes from CMG Builder for all wells QW3, QW4, QW6HD1, QW6HD2, QW6HD3 and QW7 were that the calculated skin factor from the Analytical correlation would result in a negative well index. This correlation will not be used in the calculation of average well index. However, it was noted that other correlation results may still be used if they result in positive well indices.

The Full field model(FFM) reservoir model and our single well modeling described above does not indicate any problems with condensate banking or blockage in the horizontal wellbore configuration. However, the radial flow of condensate in higher condensate mobility reservoirs has been shown to be problematic lending to the possible conclusion that horizontally completed wells will result in less problems with condensate blocking near the wellbore. 


\section{Evaluation of Mixing Effects and in the reservoir}

The gas composition graphs of the wells around observation well QE2 and injection withdraw wells QW3 show the QW2 has went from $78.6 \% \mathrm{CH} 4$ to $87.0 \% \mathrm{CH} 4$ due to the five simulated storage cycles. The gas composition around QW3 active injection and withdrawal well has been less dramatic going from $94.0 \% \mathrm{CH} 4$ to $96 \% \mathrm{CH} 4$. This compares favorbly with the actual gas samples taken on thee wells over the last several years. This graph in Figure $\mathbf{5 . 1 3}$ further helps in understanding the mixing results of the storage cycles:

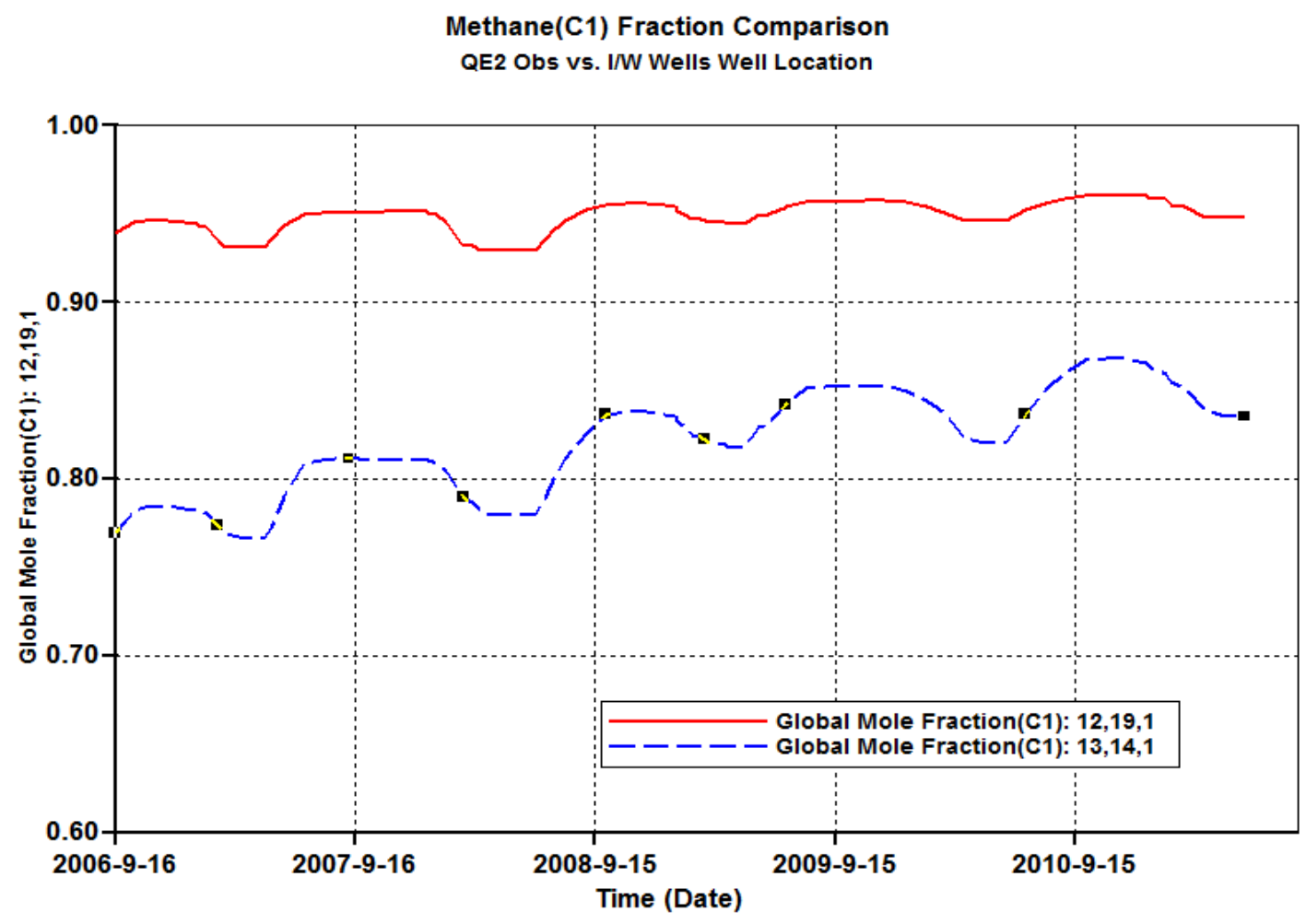

Figure 5.13: Comparison of $\mathrm{CH} 4$ concentrations changes due to Storage Operations 


\section{Storage Operational Design Evaluation}

One of the problems with accurate condensate prediction is validation of the reservoir simulator rate outputs with actual collected field data. Inaccuracies with field data usually arise from inadequate measuring and handling. A major problem with the measurement in a gas condensate two phase system is the well stream contains liquid and gas. Therefore, a specialized system is required to break the well stream out and stabilize them until equilibriums are reached and then pass them through measurement. These type systems are customarily designed for production operations. Storage operations typically do not require such systems and are not always given proper consideration.

Aminian et al (SPE 111193 2007) stated that the efficiency of the liquid separation facilities at surface could contribute to the loss of liquid to gas phase. If the separation facilities reach the maximum recovery capability at lower gas rates, the increase in rate will not result in additional liquid recovery and the liquid yield appears to drop off. To investigate this concept, the liquid yields were re-calculated based on constant gas rates. Figure 5.9 illustrate the revised liquid yields based on two different constant withdrawal rates. As it can be seen, the revised yields are well within the predicted ranges by the model. It should however be noted that there are no independent evidence available to verify if this phenomenon is occurring in the field.

As can be seen from Figure 5.8, although heterogeneities altered the liquid yield predictions, they could not account for sensitivity of the liquid yields to flow rate.

The model does not account for pressure drop in the wellbore and pressure drop in the wellbore could result in liquid drop out and accumulation in the wellbore. This is particularly true for horizontal/inclined wells. The pressure drop, and as the consequence the liquid drop out, in the wellbore is flow rate dependent. However, the limited volumes of the wellbores do not allow for significant liquid accumulations. This could provide only a partial explanation for lower liquid recovery at the surface. 
The liquid production was estimated based on liquid level collected in the storage tank and as a result was not very accurate. Furthermore, the temperature variation may have contributed to loss of the liquids to vapor phase. However, these inaccuracies do not seem to be rate dependent and as result cannot provide a justification for differences between the predicted and actual liquid yields.

The following Operational and Design Studies were broken into these two (2) areas:

1) High rate or Peaking Tests were modeled to evaluate condensate yield recoveries. High rates from storage facilities are normal operations and must be fully understood particularly where problems might interfere with the ability to meet the requirement demands of storage service

2) Simulate Realistic Surface Conditions for Condensate Yield Calibration. As mentioned earlier the surface separation facilities were not designed with high efficiency in mind.

\section{Evaluation of High Rate and Peaking Tests and Condensate Recovery}

Understanding that when the rates are increased the model predicted condensate yields decrease and when the rates are reduced the condensate yields begin to increase. This part of the study reviewed the results of rate changes and high peaking periods to evaluate the affect on condensate yields.

During the 08-09 withdrawal period in January 09 a test was performed for seven (7) days where an initial 130 mmscfd was withdrawn from the field followed by a higher rate of 200 mmscfd for 4 days. This accounted for approximately 1.064 BCF of gas withdrawn over the test period. The total amount of condensate produced for the 08-09 season was 5890 bbls. The yield for the season was much lower than previous years. These liquid rate reductions were much greater than the natural historical decline due to the condensate saturations being reduced from continued storage cycles. It was thought that the condensate recoveries have been affected by the high rates during the test and in normal storage operations where minor peaking opportunities are exploited. 
To analyze this with the model, two (2) withdrawal schedules were developed for the 09-10 and 10-11 withdrawal seasons. The peaking rate produced 1503 bbls or $19.6 \%$ less than the constant rate case. The results are summarized in Table 5.4 and Figure 5.14 below:

\begin{tabular}{|c|c|c|c|c|c|c|}
\hline Season & MMSCFD & $\begin{array}{c}\text { MMSCFD } \\
\text { per well }\end{array}$ & Days & $\begin{array}{c}\text { Total Gas } \\
\text { MMSCF }\end{array}$ & $\begin{array}{c}\text { Cumulative } \\
\text { MMSCF }\end{array}$ & $\begin{array}{c}\text { Condensate } \\
\text { Production } \\
\text { BBLS }\end{array}$ \\
\hline $09-10$ & 34.8 & 5.5 & 120 & 4176 & 3960 & 7681 \\
\hline $10-11$ & $\begin{array}{c}33.6 \\
150.0 \\
\text { SHUT IN } \\
150.0 \\
\text { SHUT IN } \\
33.6\end{array}$ & $\begin{array}{c}5.6 \\
25.0 \\
0.0 \\
25.0 \\
0.0 \\
5.6\end{array}$ & $\begin{array}{c}30 \\
7 \\
23 \\
7 \\
23 \\
30\end{array}$ & $\begin{array}{r}1008 \\
1050 \\
1050 \\
1008\end{array}$ & 4116 & 6178 \\
\hline & & & & & DIFF & 1503 \\
\hline
\end{tabular}

Table 5.4. Results of High Rate Peaking Schedules Scenarios

Withdrawal Rate Yield Comparison

09-10 Constant vs. 10-11 Peaking

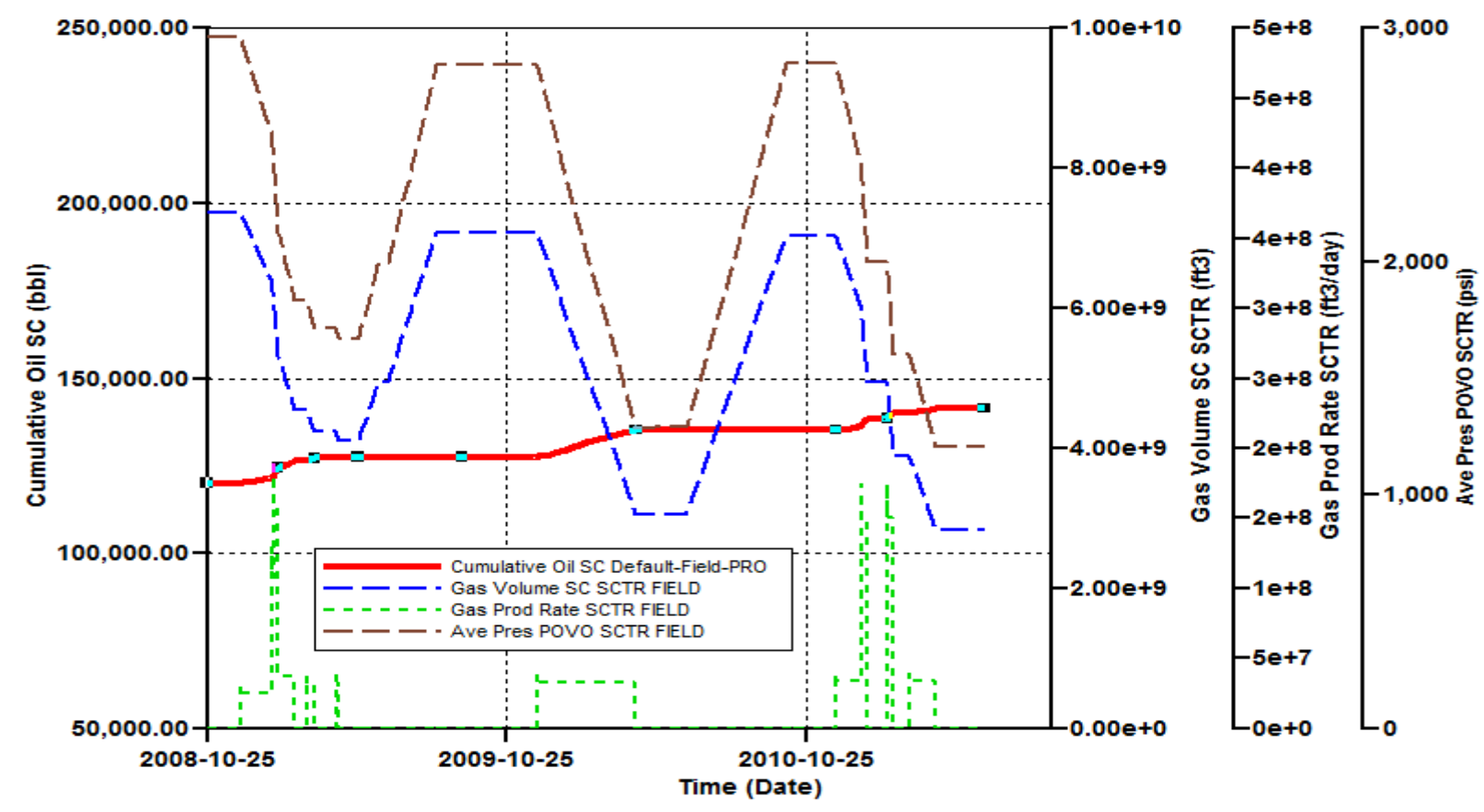

Figure 5.14: Peaking vs. Constant Withdrawal Rate Yield Comparison 


\section{Evaluation of Surface Facility Efficiency}

The table below Table $\mathbf{5 . 5}$ shows how the separator conditions affect the ability of the reservoir to model to predict the actual storage operations. Since maximizing condensate production was not the original intent of the project, the facilities installed concentrated on storage operations. The separator conditions that exist in the field do not exist where the gas and liquid phases can reach equilibrium and thus generate accurate production volume streams. In the field the facilities consist of a heater and regulator to reduce the pressure, a filter separator that has a pressure drop across it and the solid desiccant de-hydration system. All of these devices or facilities cause liquids to drop out either by cooling of the gas or reducing the pressure.

SEPARATOR EFFICIENCY STUDY

\begin{tabular}{|c|c|c|c|c|c|c|c|c|c|}
\hline & $\begin{array}{c}\text { Separator } \\
\text { Pressure } \\
\text { (psi) }\end{array}$ & $\begin{array}{c}\text { TEMP } \\
\text { F }\end{array}$ & DATE & $\begin{array}{c}\text { Ave Pres } \\
\text { HC POVO } \\
\text { SCTR (psi) }\end{array}$ & $\begin{array}{c}\text { Actual Ave } \\
\text { Pres HC } \\
\text { POVO } \\
\text { SCTR } \\
\text { (psi) }\end{array}$ & $\begin{array}{c}\text { Gas Oil } \\
\text { Ratio } \\
\text { SCTR } \\
\text { (ft3/bbl) }\end{array}$ & $\begin{array}{c}\text { Actual Gas } \\
\text { Oil Ratio } \\
\text { SCTR } \\
\text { (ft3/bbl) }\end{array}$ & $\begin{array}{c}\text { Predicted } \\
\text { Cumulative } \\
\text { Oil (bbls) }\end{array}$ & $\begin{array}{c}\text { Actual } \\
\text { Cumulative } \\
\text { Oil (bbls) }\end{array}$ \\
\hline Case 1 & 1100 & 50 & $4 / 1 / 2010$ & 1706 & 1095 & 0 & 0 & 133327 & 123815 \\
\hline Case 2 & 1500 & 50 & $4 / 1 / 2010$ & 1710 & 1095 & 0 & 0 & 122004 & 123815 \\
\hline Case 3 & 1100 & 60 & $4 / 1 / 2010$ & 1707 & 1095 & 0 & 0 & 129799 & 123815 \\
\hline Case 4 & 1000 & 60 & $4 / 1 / 2010$ & 1707 & 1095 & 0 & 0 & 131793 & 123815 \\
\hline
\end{tabular}

Table 5.5. Separator Efficiency Evaluation Results

The best match from the model was Case 2 where the separator conditions indicated that 1500 psi and $50 \mathrm{~F}$. would be required to match the actual cumulative condensate recovery of 123815 bbls through 2010 storage operations. The actual separator condition operates at approximately 1000 psi and 50-60 F.

This study supports that when peaking rate schedules are followed the separator efficiency is reduced causing the reduced condensate yields. When the rates are constant the separation facilities allows the liquids and gas streams to reach equilibrium and increase condensate recovery. 
The temperature plays a significant part but is hard to monitor in the field due to heat transfer processes in the flowing mass thru the facilities. The temperature is shown above to have a 4000 bbls increase over four(4) storage cycles due to dropping the temperature 10 degrees from $60 \mathrm{~F}$ to $50 \mathrm{~F}$. 


\section{CHAPTER 6 CONCLUSIONS}

1. Mixing of the lean storage gas with the rich wet native gas is a factor to consider when designing the conversion of a gas condensate production reservoir to gas storage operations.

2. The design of the surface facilities to increase the efficiency of the recovery process is critical to the storage conversion process. Without accurate accounting for the produced condensate the objectives of storage modeling efforts are diminished. It was shown through the modeling cases involving changing separator conditions that the condensate yields could be matched with actual data.

3. The wellbore design is important but not critical to the development of the storage operations. It was shown through the modeling efforts that the increase in condensate recovery in a homogeneous reservoir from horizontally completed injection withdrawal wells was minimal.

4. The reservoir modeling process indicated that in a reservoir as homogeneous as the study reservoir that adjustments made to the permeability in specific layers where able to improve condensate recovery predictions. These changes were not possible because the negative impact on the original history match.

5. This study also concluded that based on the equation of state model as predicted by the WinProp CMG application used in the compositional reservoir simulator the storage capacity size was dependent on the gas composition. As storage operations removes the wet gas and the lean storage gas occupies more and more of the pore space the increased gas compressibility reduces the reservoir capacity.

6. This research looked at storage operation designs that have not been studied and documented to the extent that could impact the storage development plan. Previous studies only looked at the cycling of gas through a sweeping action unlike the design considered in this project that utilizes the same wells for injection and withdrawal. 
7. This study indicated that it is still possible to accurately create the compositional PREOS match for use in the reservoir simulator without a suitable match for the compositional properties as compared to the laboratory studies.

8. The condensate liquid yields are rate dependent. When peaking or high rates are used the separator efficiencies declines and more liquids are carried by the gas stream to the sales point.

9. The condensate liquid yields are rate dependent. When peaking or high rates are used the separator efficiencies declines and more liquids are carried by the gas stream to the sales point.

10. The simulation results clearly indicate that complete mixing among injected pipeline gas and residue gas and liquid is not achieved during storage operations. There have been several factors discussed in this work that can influence the mixing phenomenon.

11. The Full field model(FFM) reservoir model and our single well modeling described in this doctoral work does not indicate any problems with condensate banking due to wellbore configuration. However, the radial flow of condensate in higher condensate mobility reservoirs has been shown to be problematic lending to the possible conclusion that horizontally completed wells will result in less problems with condensate blocking near the wellbore. 


\section{RECOMMENDATIONS}

1. This research should in the future enable the surface facilities to be appropriately designed so that a better estimate of condensate recovery can be accessed. Although the focus of the project was to develop a viable natural gas storage plant not to design an efficient condensate recovery extraction plant.

2. In the future when a condensate reservoir is discovered like the prospect in this research more comprehensive PVT phase behavior studies should be conducted prior to beginning production. Specifically obtaining extended gas analysis to include the C7+ constituents.

3. If a gas condensate reservoir is selected for storage development it is strongly recommended to perform phase behavior modeling and employ a compositional reservoir simulator to answer the questions about mixing and condensate recovery. 


\section{REFERENCES}

1. Katz, D. L., Herzog, R. A., and Hekim, Y.: "Predicting Yield of Revaporized Condensate in Gas Storage", SPE 10166, Proceedings of Annual SPE Technology Conference, 1981.

2. Wallbrecht, J. and Onderka, V.:" International Gas Union(IGU) Triennium 2006 - 2009 Underground Gas Storage(UGS) Work Report, presented at the $24^{\text {th }}$ World Gas Conference, Argentina 2009.

3. Survey of Underground Storage of Natural Gas in the U.S. \& Canada, 2007

4. Herzog, R.A.:"Retrograde Vaporization of Residual Condensation in Storage Field Development", Operating Section Proceedings. American Gas Association Baltimore, Maryland (1980) T187.

5. Katz, D. L.:"Overview of Phase Behavior in Oil and Gas Production", SPE 9995, presented SPE International Petroleum Exhibition and Technical Symposium , Bejing, China, March 18-26.1982.

6. Coats, K.H.: "Simulation of Gas Condensate Reservoir Performance," SPE-10512, presented at the SPE Reservoir Simulation Symposium held in New Orleans, LA, Jan 31- Feb 3.

7. Khamehchi, E. and Rashidi, F,: "Simulation of Underground Natural Gas Storage in Sarajeh Gas Field", SPE 106341-MS, Technical Symposium of Saudi Arabia Section, Daharan, Saudia Arabia ,2006.

8. Kenyon, D.E. and Behie, G.A.: "Third SPE Comparative Solution Project: Gas Cycling of Retrograde Condensate Reservoirs," JPT, Dec. 1987.

9. Firoozabadi, A. and Fahes M.:" Wettability Alteration to Intermediate Gas-Wetting in Gas-Condensate Reservoirs at High Temperatures", SPE Journal, Volume 12, Number 4, December 2007 Pages 397-407.

10. Katz D., Firoozabadi A.: "Predicting Phase Behavior of Condensate/Crude-oil Systems Using Methane Interaction Coefficients", JPT, Nov. 1978, 1949.

11. Whitson, C, Øivind F. and Yanga T.: "Gas Condensate PVT - What's Really Important and Why?" presented at the IBC Conference "Optimisation of Gas Condensate Fields", London, Jan. 28-29, 1999.

12. Whitson, C.H. and Torp, S.B.: "Evaluating Constant- Volume Depletion Data," JPT, March 1983, 610.

13. Kai Luo,K., Li, S., Zheng, X., Chen, G., Liu, N., and Sun, W.: "Experimental Investigation Into Revaporization of Retrograde Condensate" SPE 67283, paper was presented at the SPE Production and Operations Symposium held in Oklahoma City, OK March 24-27, 2001, 
14. Ahmed,T.H.,Cady,G.V and Story,A.L.: "A Generalized Correlation for Characterizing the Hydrocarbon Heavy Fractions", SPE 14266, presented at the SPE Annual Technical Conference and Exhibition held in Las Vegas, September 22-25, 1985.

15. Aminian K., Brannon, A.W., and Ameri, S.: "Evaluation of a Depleted Gas Condensate Reservoir for Gas Storage" SPE 91483, Proceedings of SPE Eastern Regional Conference, Charleston, WV, 2004.

16. Aminian, K.: "Phase -Equilibrium and Reservoir Depletion Calculation on PC Using the Peng-Robinson Equation of State", SPE Computer Applications, May-June 1989, 20.

17. Van Tyne, A.M., "Subsurface Onondaga Reefs", AAPG Search and Discovery Article \#90954, 1995 AAPG Eastern Section, Schenectady, New York.

18. Aminian, K. Brannon, A. and Ameri, S.: "Gas Storage in a Depleted Gas/Condensate Reservoir in the Appalachian Basin", SPE 104555, Proceedings of SPE Eastern Regional Conference, Canton, Ohio ,2006.

19. Aminian , K., Brannon , A. W., Ameri , S. and Bhasvar, A.: "Evaluation of the Results of Gas Storage in a Gas/Condensate Reservoir in the Appalachian Basin" , SPE 111193,Proceedings of SPE Eastern Regional Conference, Lexington, Ky, 2007.

20. Gringarten, A.C. , Bozorgzadeh, M. , , Daungkaew, S. and Hashemi , A.:" Well Test Analysis in Lean Gas Condensate Reservoirs: Theory and Practice ", SPE100993-MS, presented at the 2006 SPE Russian Oil and Gas Technical Conference and Exhibition held in Moscow, Russia, October 3-6, 2006.

21. Gringarten, A.C. Hashemi , A. and Nicholas, L.M:" Well Test Analysis of Horizontal Wells in Gas/Condensate Reservoirs", SPE 89905-PA, February 2006 SPE Reservoir Evaluation and Engineering.

22. Qing Sun, S. and Sloan, R. "Quantification of Uncertainty in Recovery Efficiency Predictions: Lessons Learned from 250 Mature Carbonate Fields", SPE 84459-MS, presented at the SPE Annual Technical Conference and Exhibition held in Denver, Colorado. October 5-8, 2003.

23. Peng, D.Y. and Robinson, D. B.: "A New Two-Constant Equation of State", Ind. Eng. Chem. Fundamentals (1976), 15, No. 1, 54.

24. Whitson, C.: "Characterizing Hydrocarbon Plus Fractions," SPEJ, 23, 1983, 683.

25. Montel, F. and Gouel, P.L.: "A New Lumping Scheme of Analytical Data for Composition Studies," paper SPE 13119 presented at the 1984 SPE Annual Technical Conference and Exhibition held in Houston, September 16-19.

26. Coats, K.H. and Smart, G.T.: "Application of a Regression-Based EOS PVT Program to Laboratory Data," SPE Reservoir Engineering, May 1986, 277.

27. SPE Reservoir Engineering Research Institiute and Mashhad Fahes. Imperial College Presented in paper from December 2007 SPE Journal Page 407 “ 
28. Fan, L., Whitson, C. H. and Harris, B.:" "Understanding Gas Condensate Reservoirs" ,Schlumberger Oilfield Review, Winter 2005-06 


\section{APPENDICES}

Figure 1. Initial Oil Saturation at block 14,20,1 at Dewpoint Pressure

Figure 2. Oil Saturation near QE2 after two years production block address $13,14,1$

Figure 3. Oil Saturation Maximum around QE2 after seven years production block address $14,14,1$

Figure 4. Oil Saturation around I/W wells at beginning of first storage injection cycle $14,20,1$

Figure 5. Oil Saturation around I/W wells at end of first storage injection cycle $14,20,1$

Figure 6. Oil Saturation around I/W wells at end of first storage withdrawal cycle $14,20,1$

Figure 7. Oil Saturation around I/W wells at end of second storage injection cycle 14,20,1

Figure 8. Pressure at the beginning of primary production $14,20,1$

Figure 9. Pressure at the end of primary production $14,20,1$

Figure 10. Pressure at the end of first storage injection cycle 14, 20,1

Figure 11. Pressure at the end of first storage withdrawal cycle 14, 20,1

Figure 12. Global Methane Concentration at beginning of primary production $14,20,1$

Figure 13. Global Methane Concentration at end of primary production 14,20,1

Figure 14. Global Methane Concentration at end of first storage injection cycle $14,20,1$

Figure 15. Global Methane Concentration at end of first storage withdrawal cycle $14,20,1$

Figure 16. Isosurface of Oil Saturation at beginning of primary production

Figure 17. Isosurface of Oil Saturation after two(2) years of primary production

Figure 18. Isosurface of Oil Saturation at after eighteen(18) years of primary production

Figure 19. Isosurface of Oil Saturation at beginning of storage

Figure 20. Isosurface of Oil Saturation at end of first storage injection cycle (note increased saturation at old production well)

Figure 21. Isosurface of Oil Saturation at end of first storage withdrawal cycle (note increased saturation at old production well)

Figure 22. Isosurface of Oil Saturation at end of second storage injection cycle (note increased saturation at old production well) 


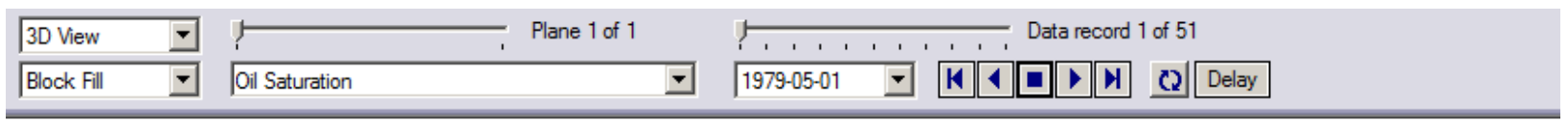

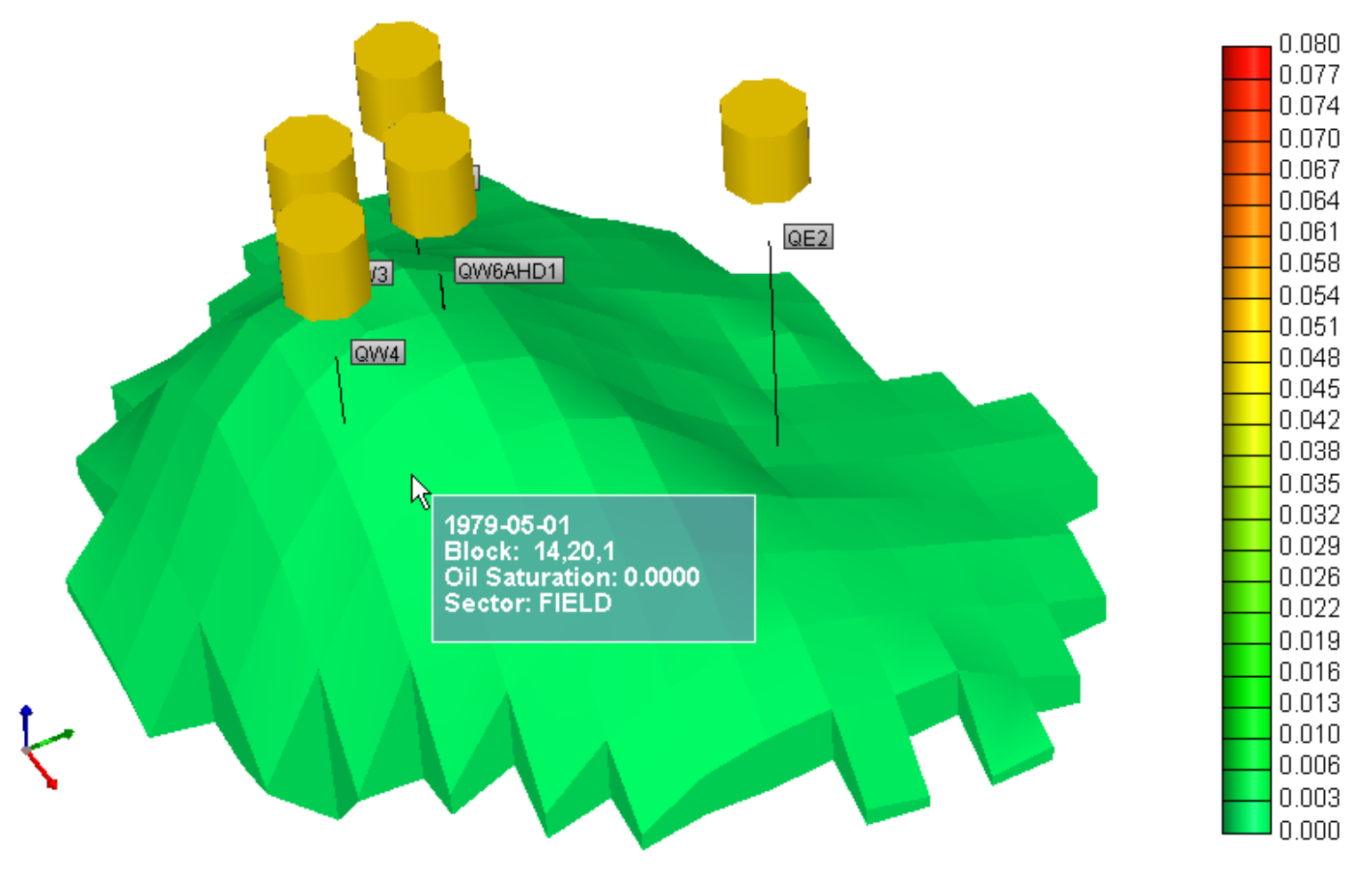

Figure 1. Initial Oil Saturation at block $14,20,1$ at Dewpoint Pressure 


BD View Plock Fill

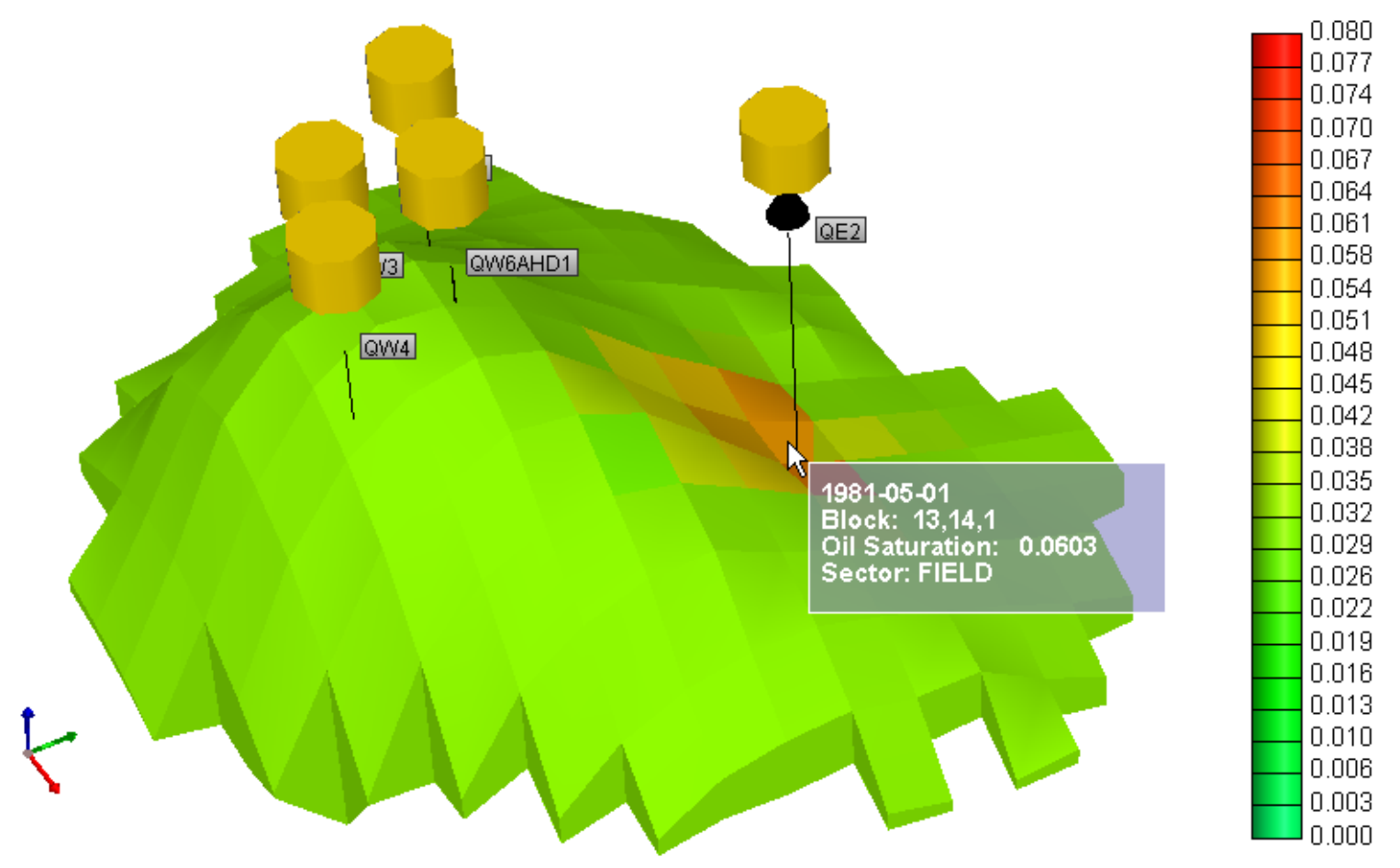

Figure 2. Oil Saturation near QE2 after two years production block address 13,14,1 


\begin{tabular}{|c|c|c|c|c|c|c|c|c|c|c|c|c|}
\hline 3D View & \multirow{2}{*}{ 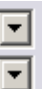 } & \multicolumn{2}{|c|}{ Plane 1 of 1} & \multicolumn{9}{|c|}{ - $, 1,1,1,1$, Data record 8 of 51} \\
\hline Block Fill & & Oil Saturation & $\nabla$ & 1986-05-01 & 7 & $\mathbf{K}$ & 4 & $\square$ & 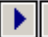 & $\mathbf{H}$ & Q & Delay \\
\hline
\end{tabular}

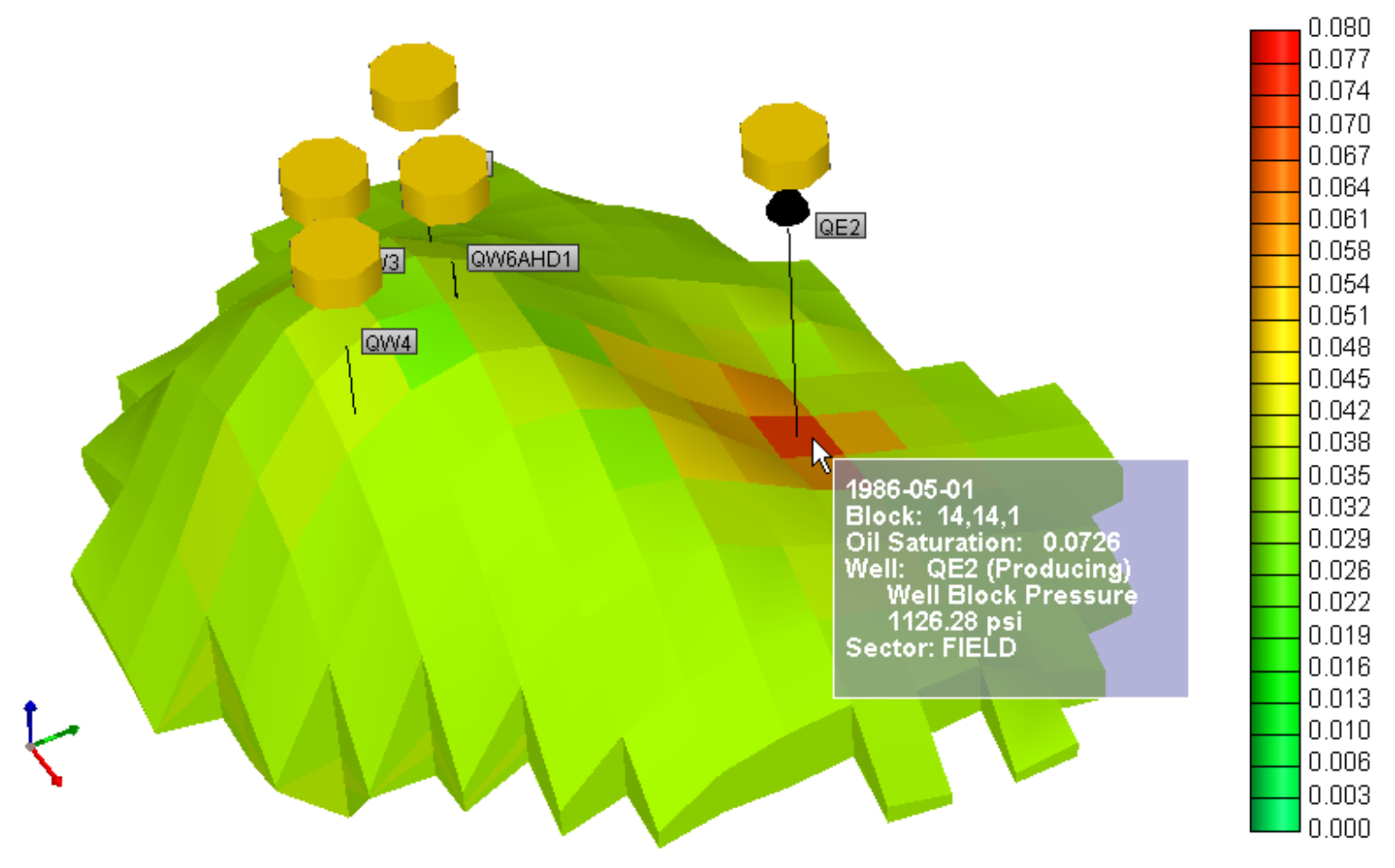

Figure 3. Oil Saturation Maximum around QE2 after seven years production block address $14,14,1$ 


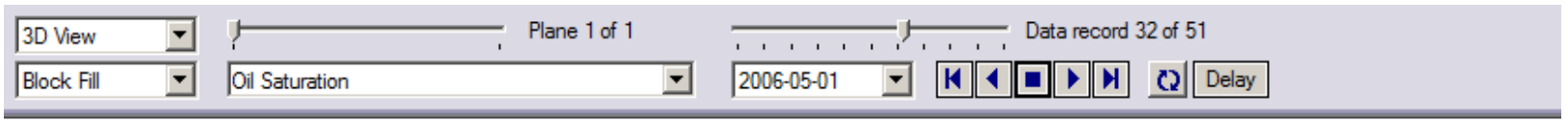

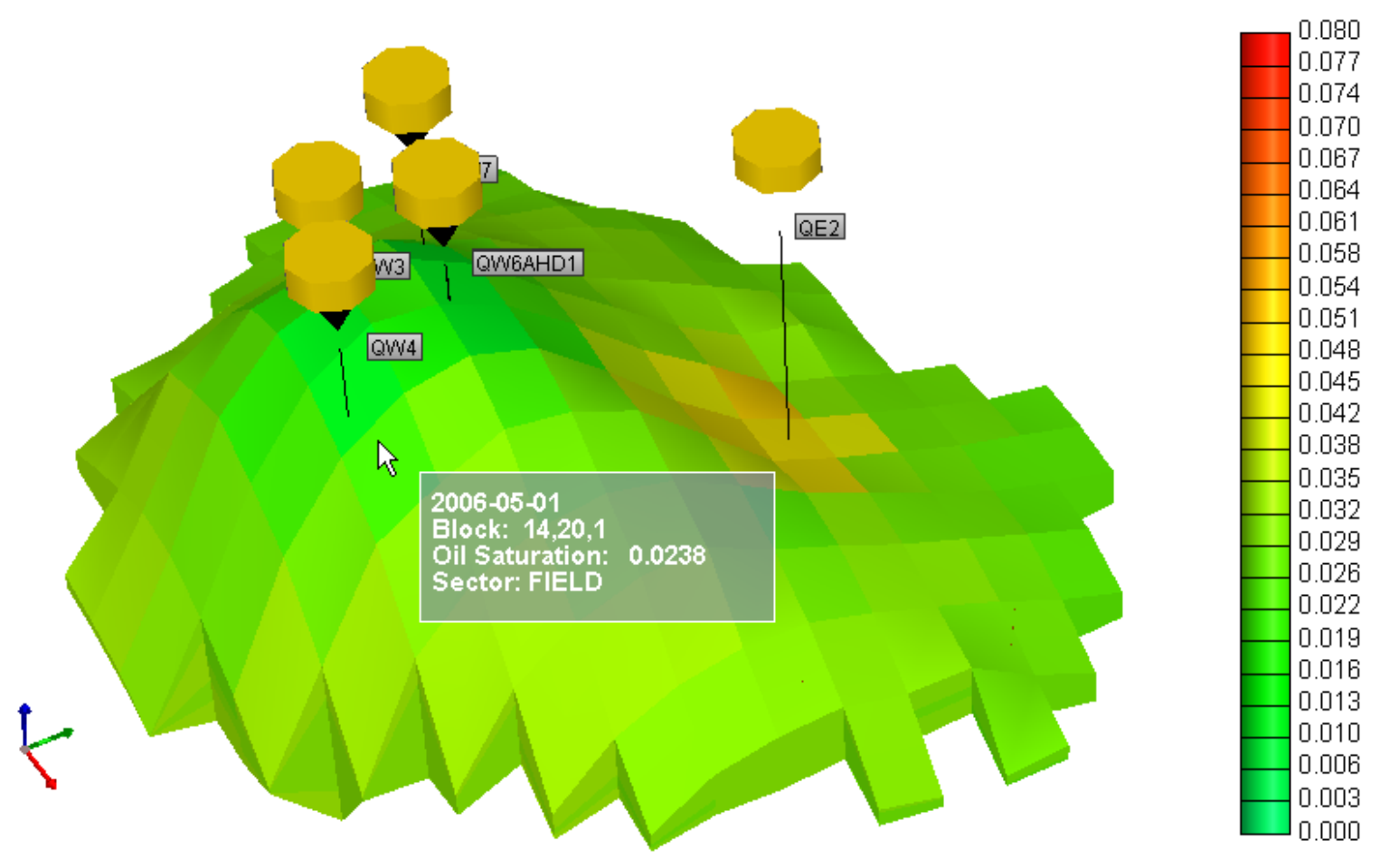

Figure 4. Oil Saturation around I/W wells at beginning of first storage injection cycle 14,20,1 


\begin{tabular}{|c|c|c|c|c|c|c|c|c|c|c|c|c|}
\hline 3D View & $\nabla$ & ! & Plane 1 of 1 & & & & & \multicolumn{5}{|c|}{ Data record 39 of 51} \\
\hline Block Fill & $\nabla$ & Oil Saturation & $\nabla$ & $2006-12-01$ & $\nabla$ & $\mathbf{K}$ & 4 & $\mathbf{\square}$ & $\nabla$ & V & $\mathrm{CQ}$ & Delay \\
\hline
\end{tabular}

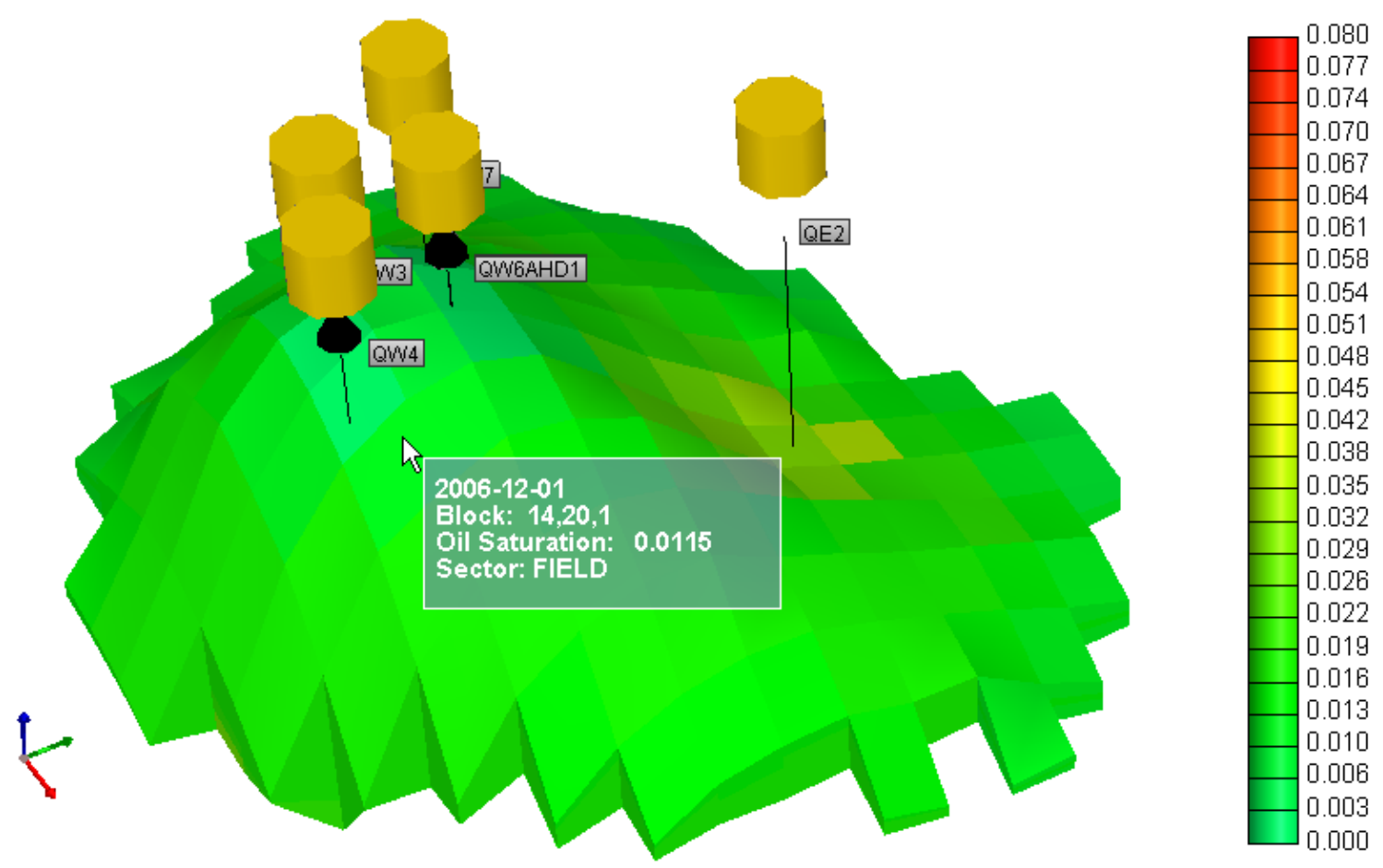

Figure 5. Oil Saturation around I/W wells at end of first storage injection cycle $14,20,1$ 


\begin{tabular}{|c|c|c|c|c|c|c|c|c|c|c|c|}
\hline \multirow[b]{2}{*}{ Block Fill } & \multirow{2}{*}{$\nabla$} & \multicolumn{2}{|c|}{ Plane 1 of 1} & \multicolumn{8}{|c|}{, , , , , ,, 1, Data record 44 of 51} \\
\hline & & Oil Saturation & $\nabla$ & 2007-05-01 & $\nabla$ & $\mathbf{K}$ & 4 & a & 11 & C) & Delay \\
\hline
\end{tabular}

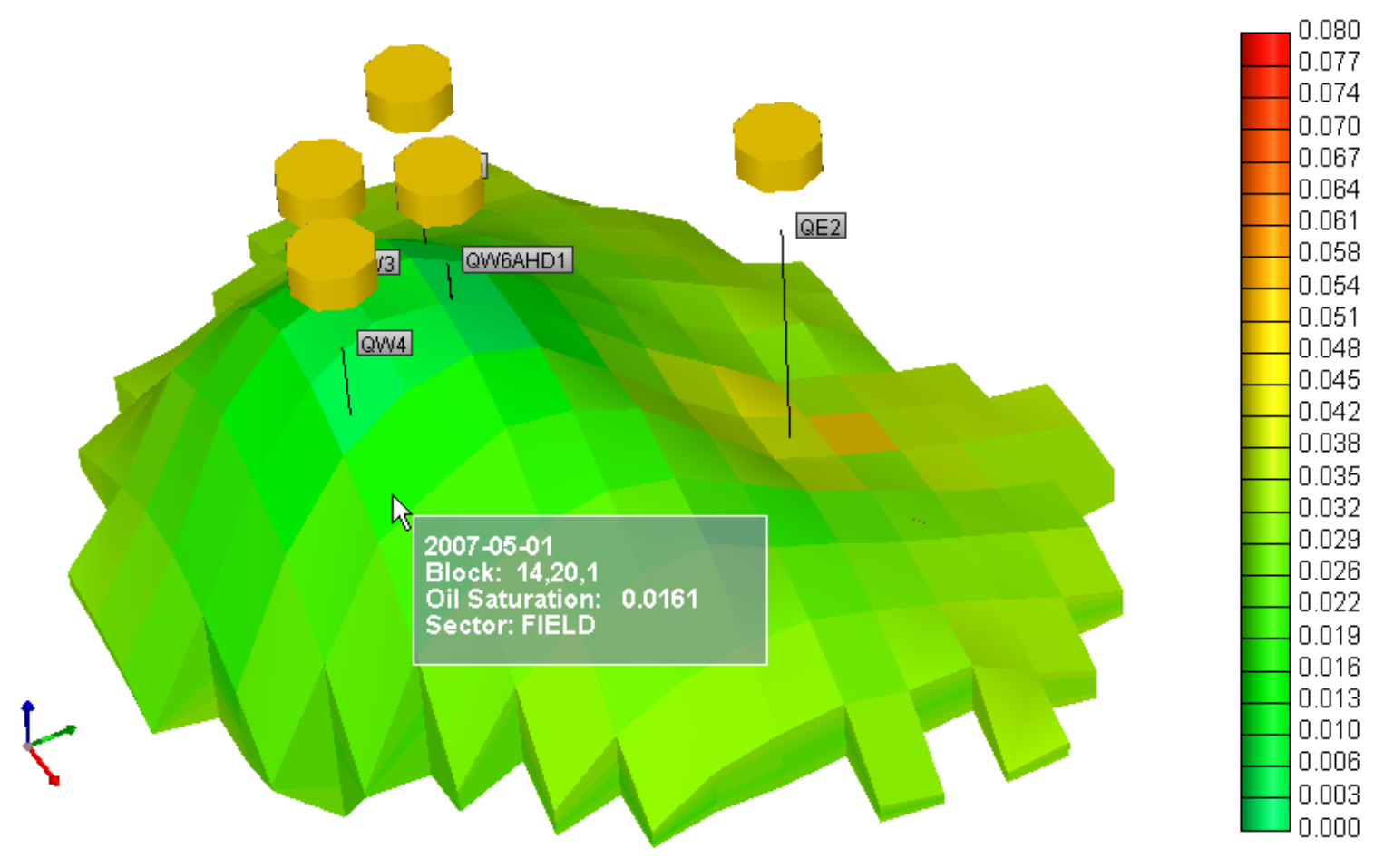

Figure 6. Oil Saturation around I/W wells at end of first storage withdrawal cycle $14,20,1$ 


\begin{tabular}{|c|c|c|c|c|c|c|c|c|c|c|c|}
\hline \multirow{2}{*}{$\begin{array}{l}\text { 3D View } \\
\text { Block Fill }\end{array}$} & \multirow{2}{*}{$\bar{r}$} & ? & Plane 1 of 1 & \multicolumn{8}{|c|}{ I $1,1,1,1,1$, Data record 51 of 51} \\
\hline & & Oil Saturation & $\nabla$ & 2007-12-01 & $\nabla$ & $\mathbf{K}$ & 4 & $\square$ & 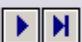 & Q & Delay \\
\hline
\end{tabular}

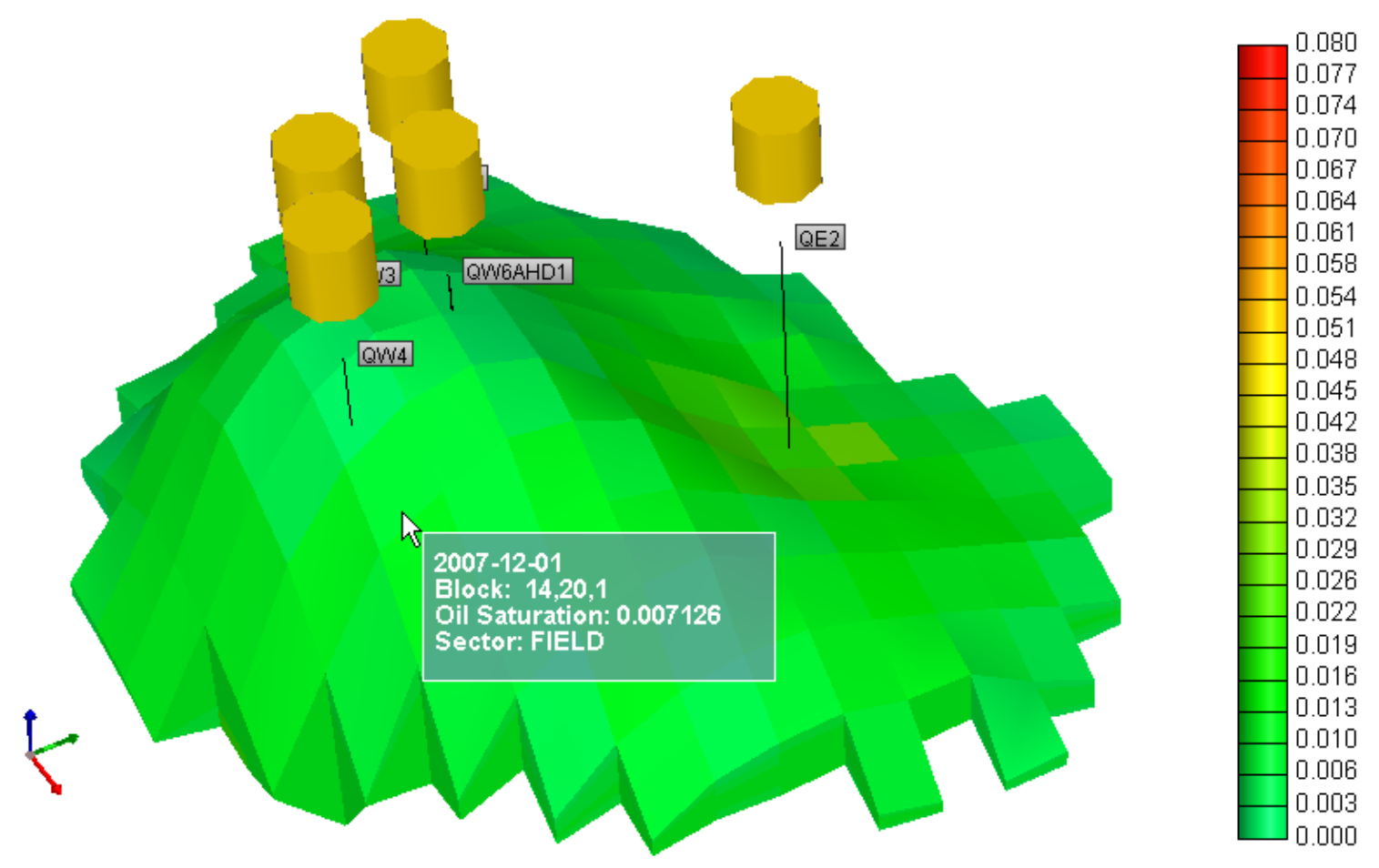

Figure 7. Oil Saturation around I/W wells at end of second storage injection cycle $14,20,1$ 


\begin{tabular}{|c|c|c|c|c|c|c|c|c|c|c|}
\hline \multirow[t]{2}{*}{$3 D V$} & \multirow{2}{*}{$\frac{7}{7}$} & \multicolumn{2}{|c|}{ Plane 1 of 1} & \multicolumn{7}{|c|}{$\sqrt{1,1,1,1,1,}$ Data record 1 of 51} \\
\hline & & Pressure & $\nabla$ & 1979-05-01 & $\mathbf{A}$ & 4 & $\square$ & 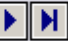 & Q & Delay \\
\hline
\end{tabular}

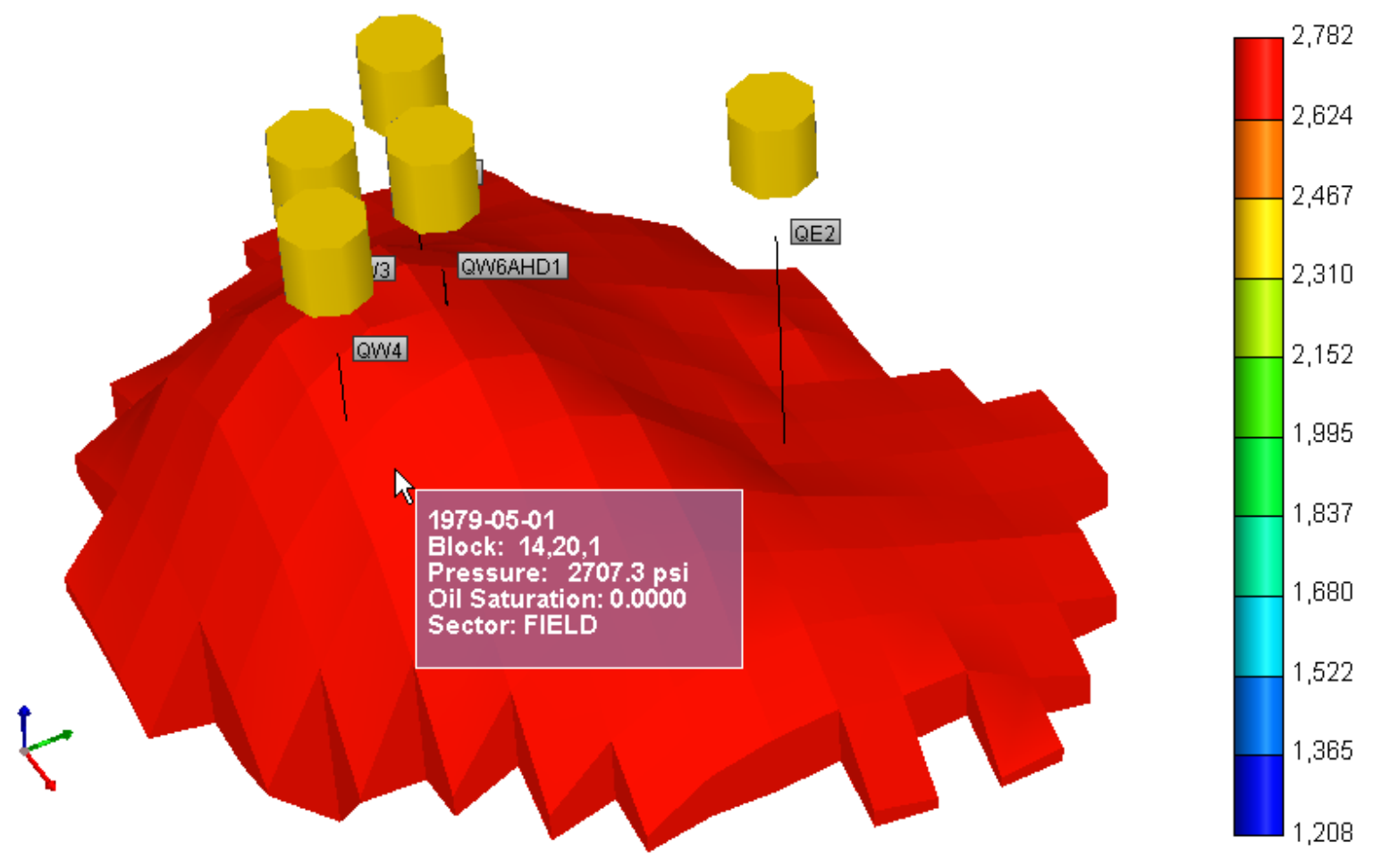

Figure 8. Pressure at the beginning of primary production 14, 20,1 


\begin{tabular}{|c|c|c|c|c|c|c|c|c|c|c|c|}
\hline \multirow{2}{*}{ 3D View } & \multirow{2}{*}{$\bar{T}$} & \multicolumn{2}{|c|}{ Plane 1 of 1} & \multicolumn{4}{|c|}{$1,1,1,1,1$} & \multicolumn{4}{|c|}{ Data record 31 of 51} \\
\hline & & Pressure & $\nabla$ & 2006-04-01 & $\nabla$ & $\mathbf{K}$ & 4 & $\square$ & H & Q $]$ & Delay \\
\hline
\end{tabular}

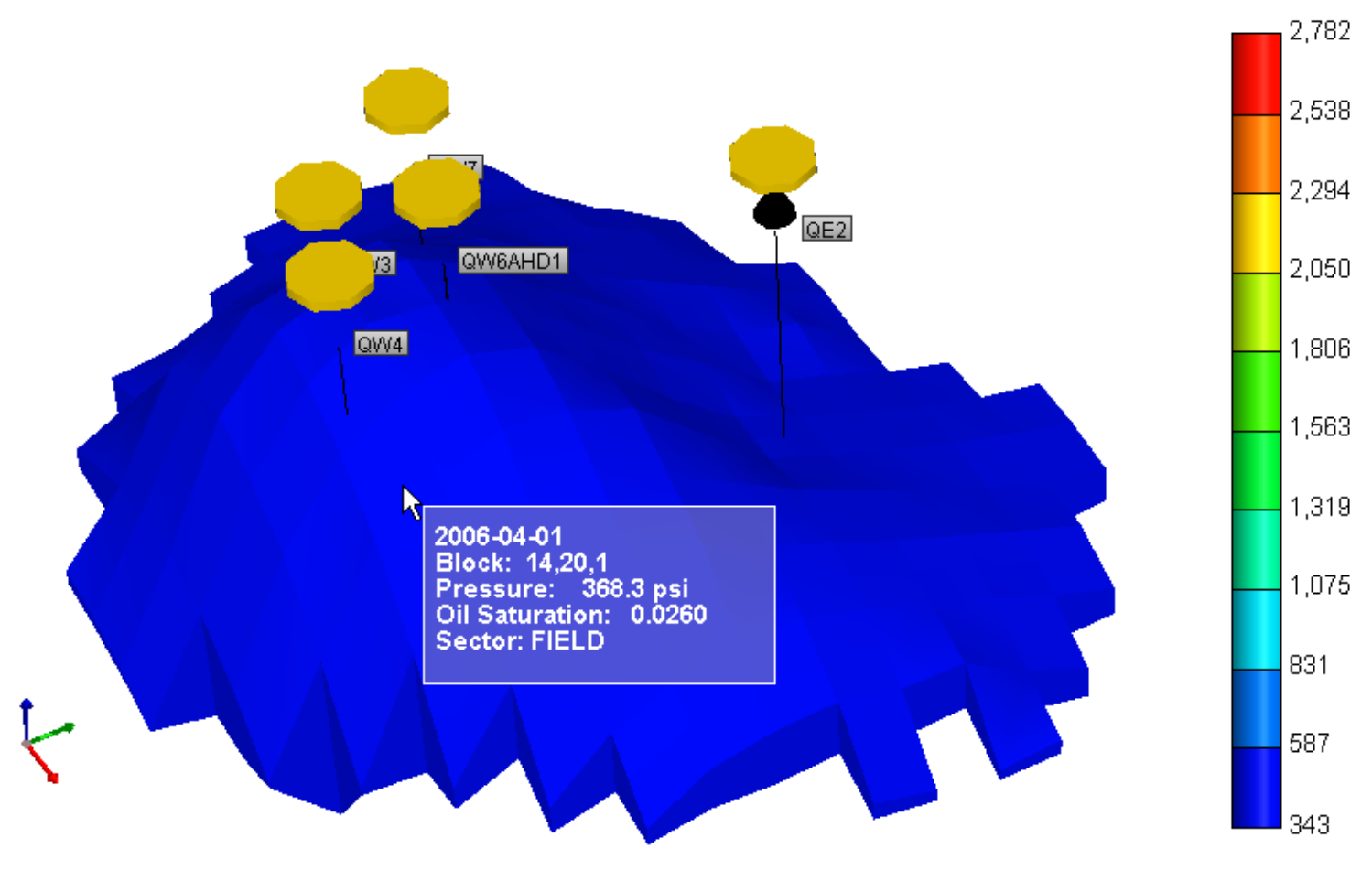

Figure 9. Pressure at the end of primary production $14,20,1$ 


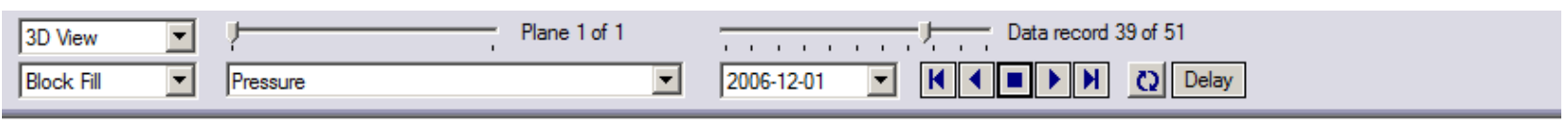

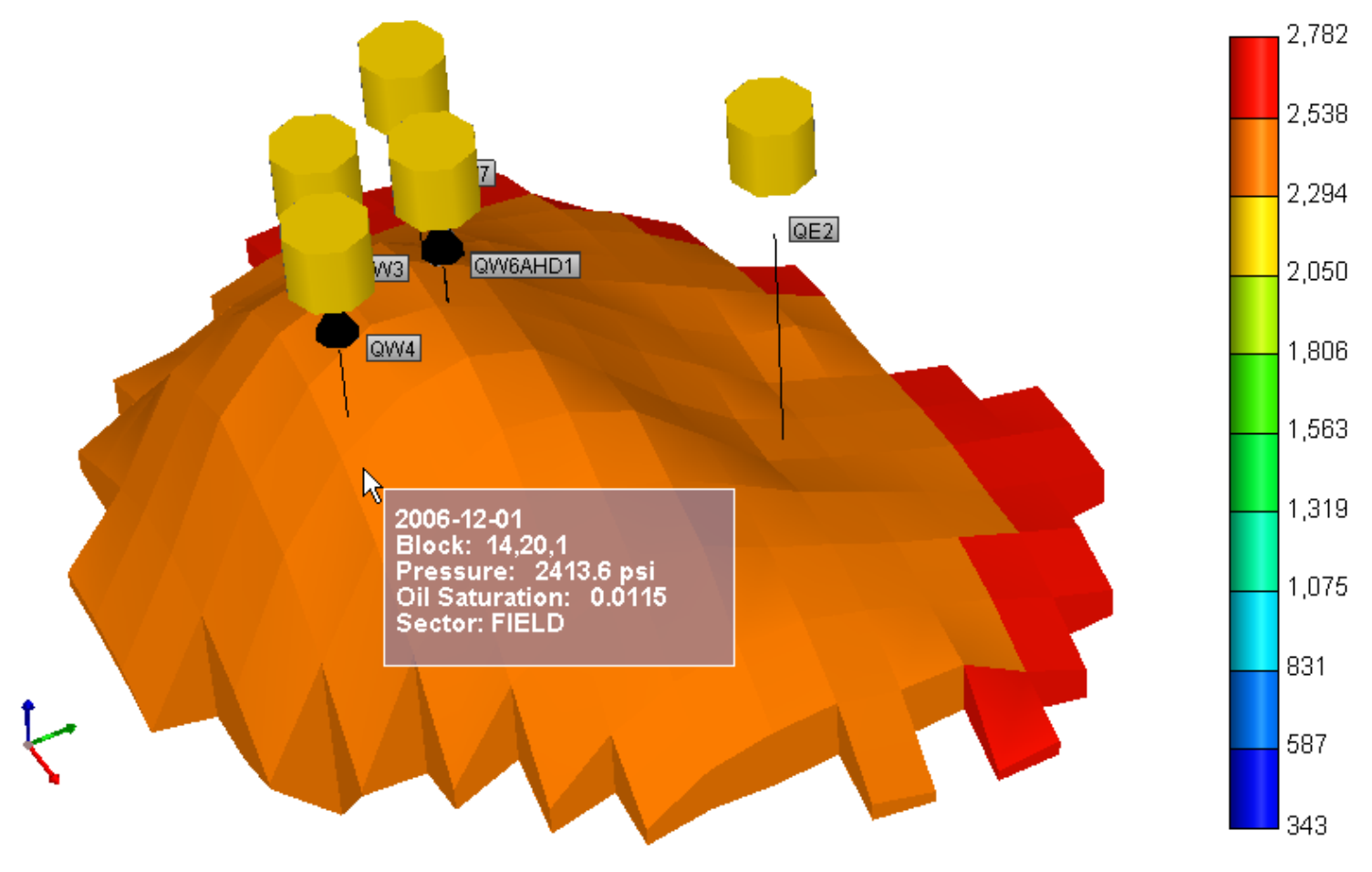

Figure 10. Pressure at the end of first storage injection cycle 14, 20,1 


\begin{tabular}{|c|c|c|c|c|c|c|c|c|c|c|c|}
\hline \multirow{2}{*}{ 3D View } & $\nabla$ & ? & Plane 1 of 1 & \multicolumn{8}{|c|}{$\overline{1,1,1,1,1,}$ Data record 43 of 51} \\
\hline & $\nabla$ & Pressure & $\nabla$ & 2007-04-01 & 7 & $\mathbf{K}$ & 4 & $\mathbf{0}$ & 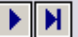 & $Q$ & \begin{tabular}{|l|} 
Delay \\
\end{tabular} \\
\hline
\end{tabular}

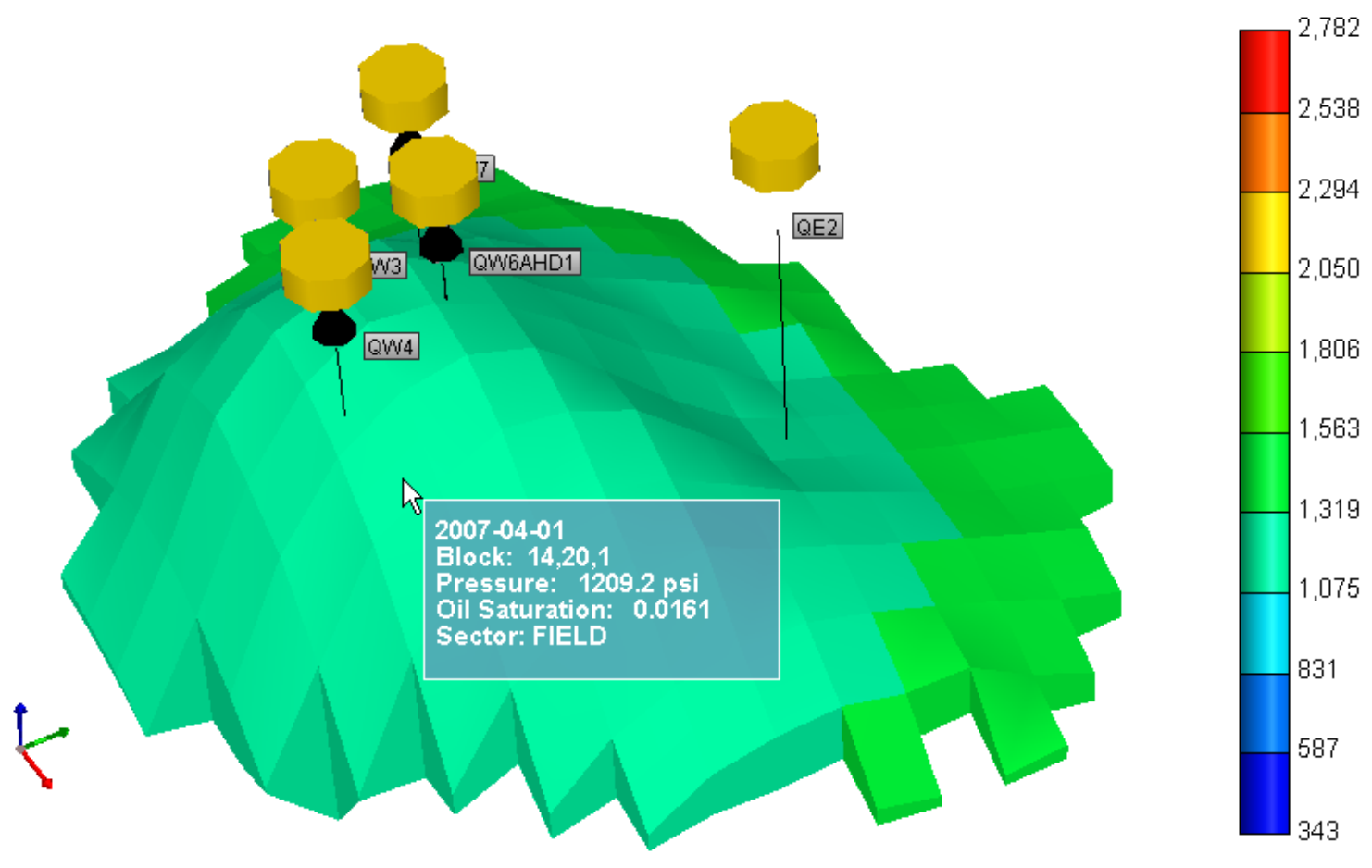

Figure 11. Pressure at the end of first storage withdrawal cycle 14, 20,1 


\begin{tabular}{|c|c|c|c|c|c|c|c|c|c|c|}
\hline 3D View & \pm & ? & Plane 1 of 1 & r., & . & & Data & recor & ord 1 of 51 & \\
\hline Block Fill & 7 & Global Mole Fraction(C1) & $\nabla$ & 1979-05-01 & $\mathbf{H}$ & 1 & a & $\nabla$ & $Q$ & Delay \\
\hline
\end{tabular}

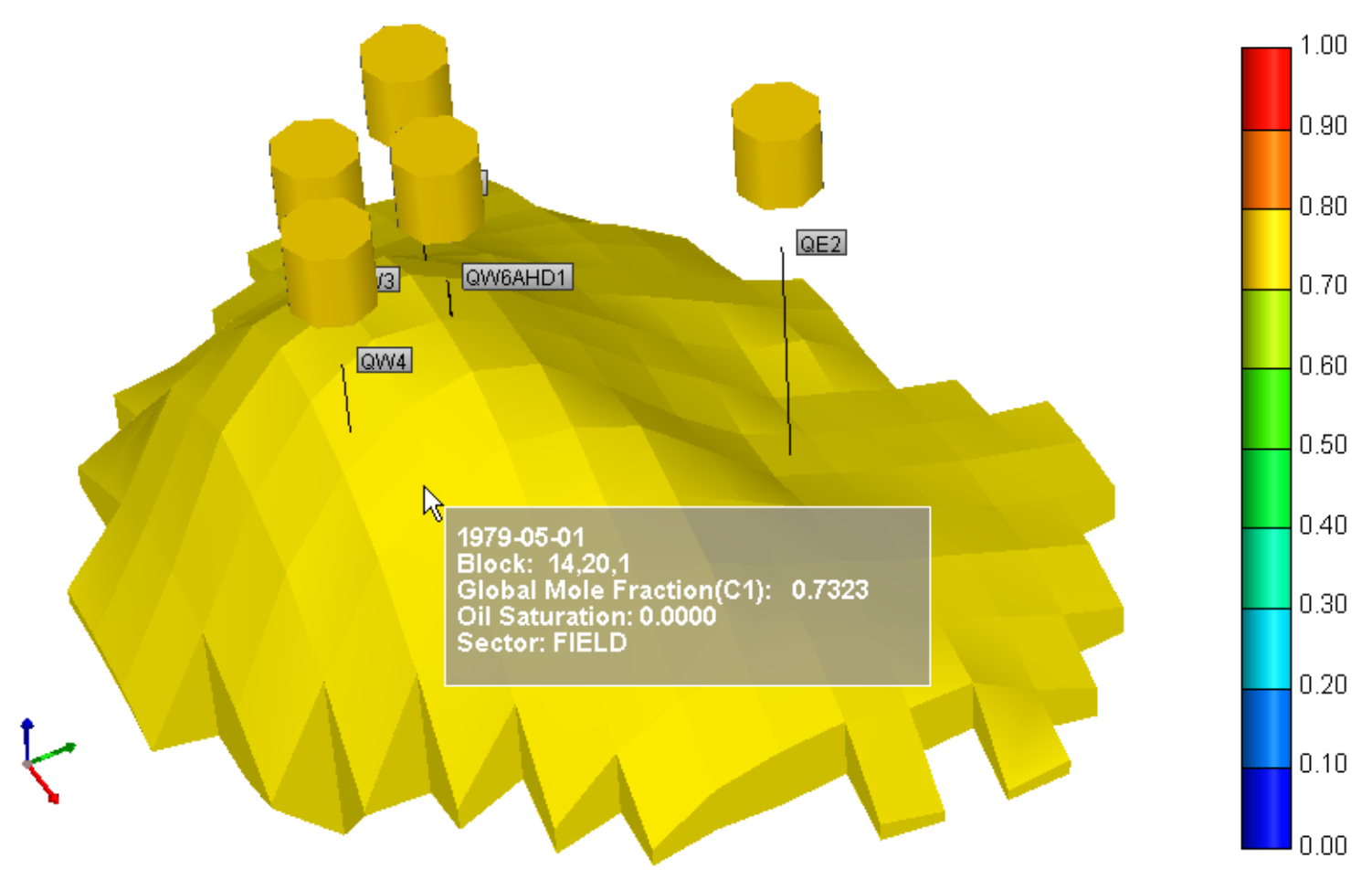

Figure 12. Global Methane Concentration at beginning of primary production $14,20,1$ 


\begin{tabular}{|c|c|c|c|c|c|c|c|c|c|c|}
\hline 3D View & $\nabla$ & - & Plane 1 of 1 & 1,1,1! & 1. & & $\mathrm{Da}$ & ta record & id 31 of 5 & \\
\hline Block Fill & $\nabla$ & Global Mole Fraction(C1) & $\nabla$ & 2006-04-01 & $\mathbf{K}$ & 4 & 口 & 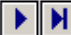 & $Q \mid[$ & \begin{tabular}{|l|} 
Delay \\
\end{tabular} \\
\hline
\end{tabular}

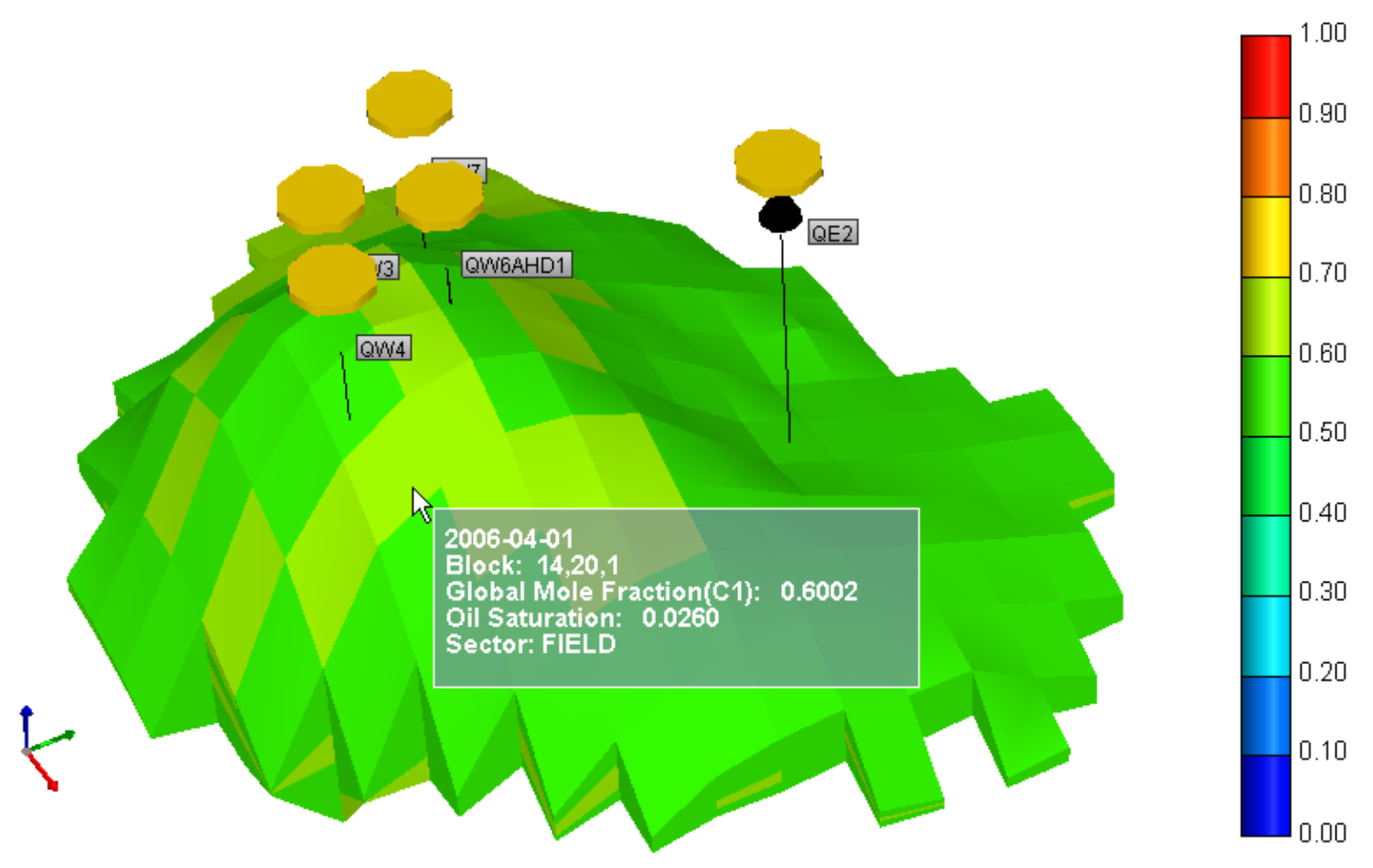

Figure 13. Global Methane Concentration at end of primary production 14,20,1 


\begin{tabular}{|c|c|c|c|c|c|c|c|c|c|c|}
\hline 3D View & $\nabla$ & ? & Plane 1 of 1 & $1,1,1$, & 上 & & Data & record & 9 of 5 & \\
\hline Block Fill & 7 & Global Mole Fraction(C1) & 7 & 2006-12-01 & $\mathbf{K}$ & 4 & 田 & 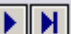 & Q & Delay \\
\hline
\end{tabular}

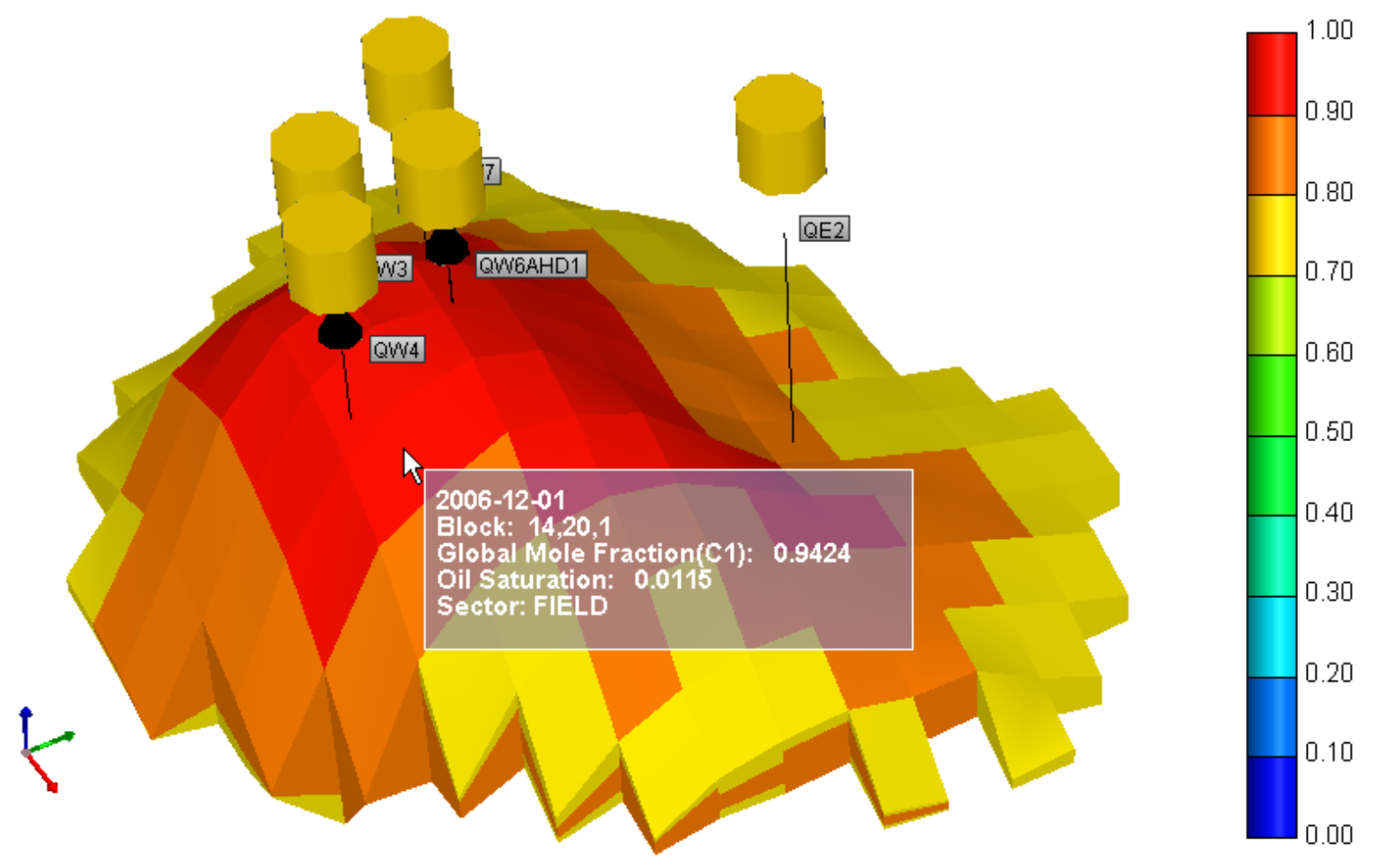

Figure 14. Global Methane Concentration at end of first storage injection cycle $14,20,1$ 


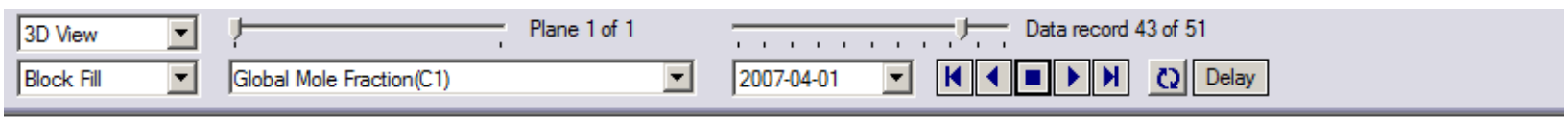

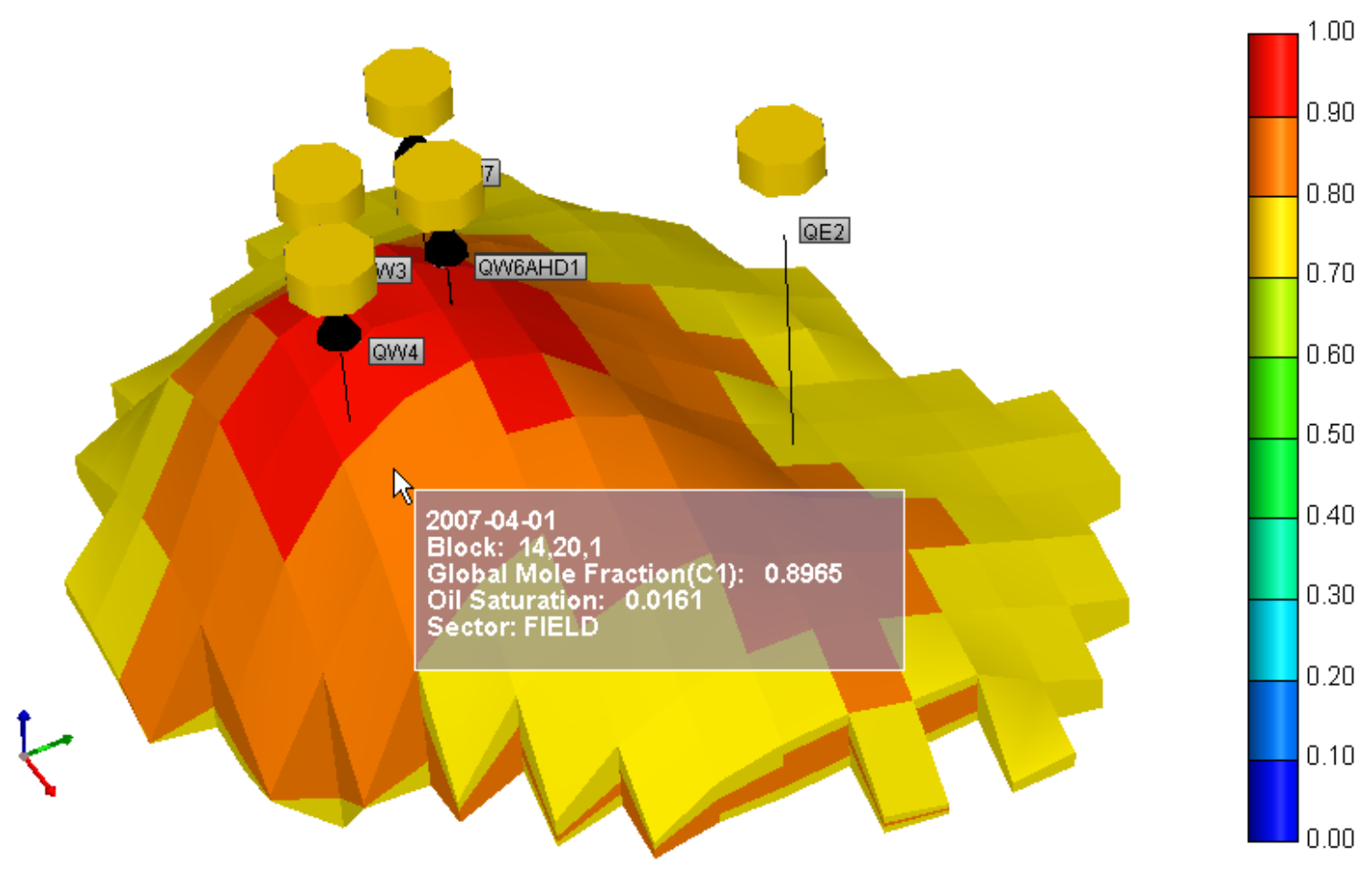

Figure 15. Global Methane Concentration at end of first storage withdrawal cycle $14,20,1$ 


\begin{tabular}{|c|c|c|c|c|c|c|c|c|c|c|c|c|}
\hline 3D View & $\nabla$ & r & Plane 1 of 1 & $\because$ & & & & Date & a rec & cord & 2 of 51 & \\
\hline Block Fill & $\nabla$ & Oil Saturation & $\nabla$ & 1980-05-01 & 7 & $\mathbf{K}$ & 4 & $\square$ & $\nabla$ & Y & $\mathbf{Q}$ & Delay \\
\hline
\end{tabular}

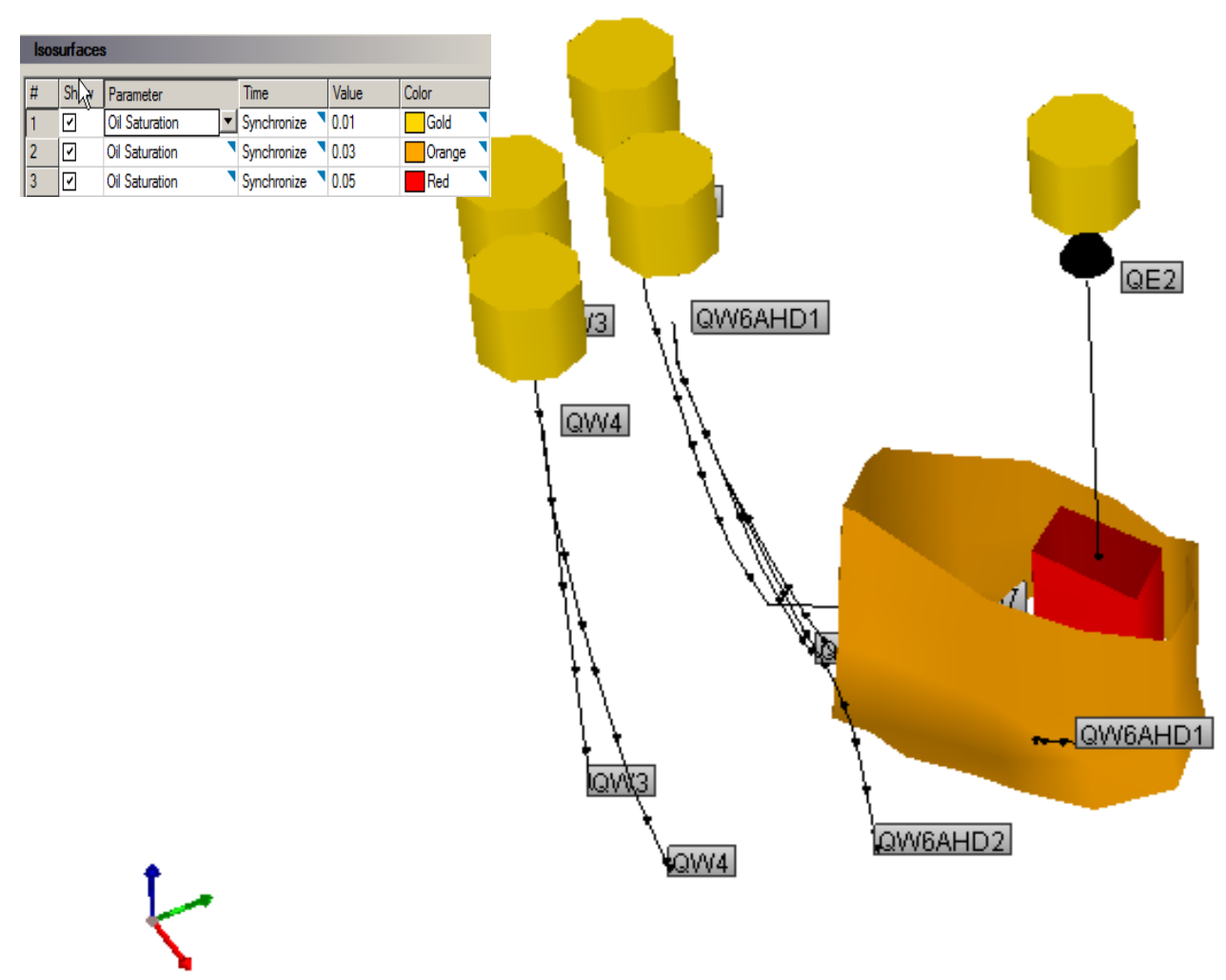

Figure 16. Isosurface of Oil Saturation at beginning of primary production 


\begin{tabular}{|c|c|c|c|c|c|c|c|c|c|c|c|c|}
\hline 3D View & \pm & F & Plane 1 of 1 & -上 & & & & & a rec & cord & of 51 & \\
\hline Block Fill & 7 & Oil Saturation & $\nabla$ & 1981-05-01 & 7 & $\mathbf{K}$ & 4 & $\square$ & $\nabla$ & $\mathbf{H}$ & $\mathbf{C}$ & Delay \\
\hline
\end{tabular}

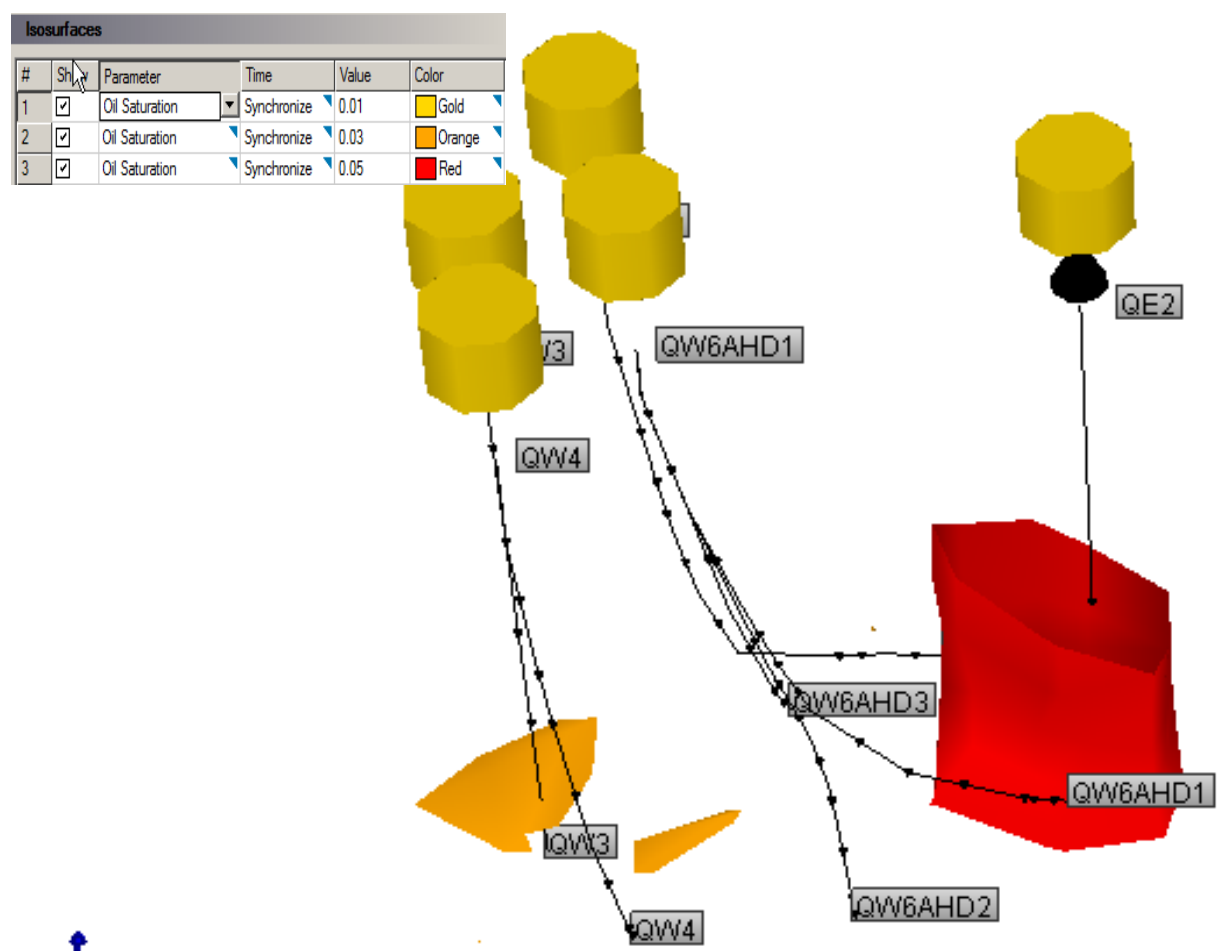

Figure 17. Isosurface of Oil Saturation after two(2) years of primary production 


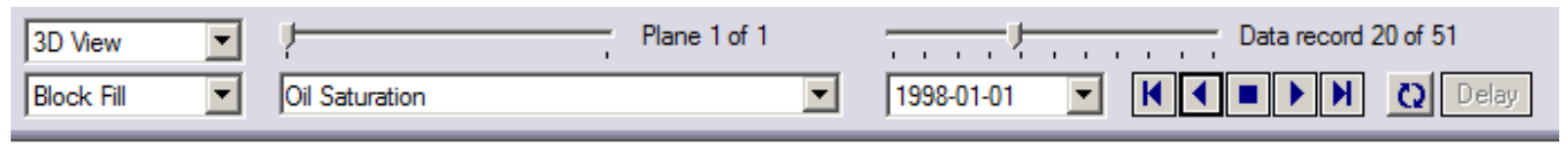

Isosurfaces

\begin{tabular}{|c|c|c|c|c|c|}
\hline & Shlh & Parameter & Time & Value & Color \\
\hline & $\nabla$ & Oil Saturation & > Synchronize & 0.01 & $\square$ Gold \\
\hline & $\nabla$ & Oil Saturation & Synchronize & $\top_{0.03}$ & Jorange \\
\hline 3 & $\nabla$ & Oil Saturation & Synchronize & ' 0.05 & Red \\
\hline
\end{tabular}

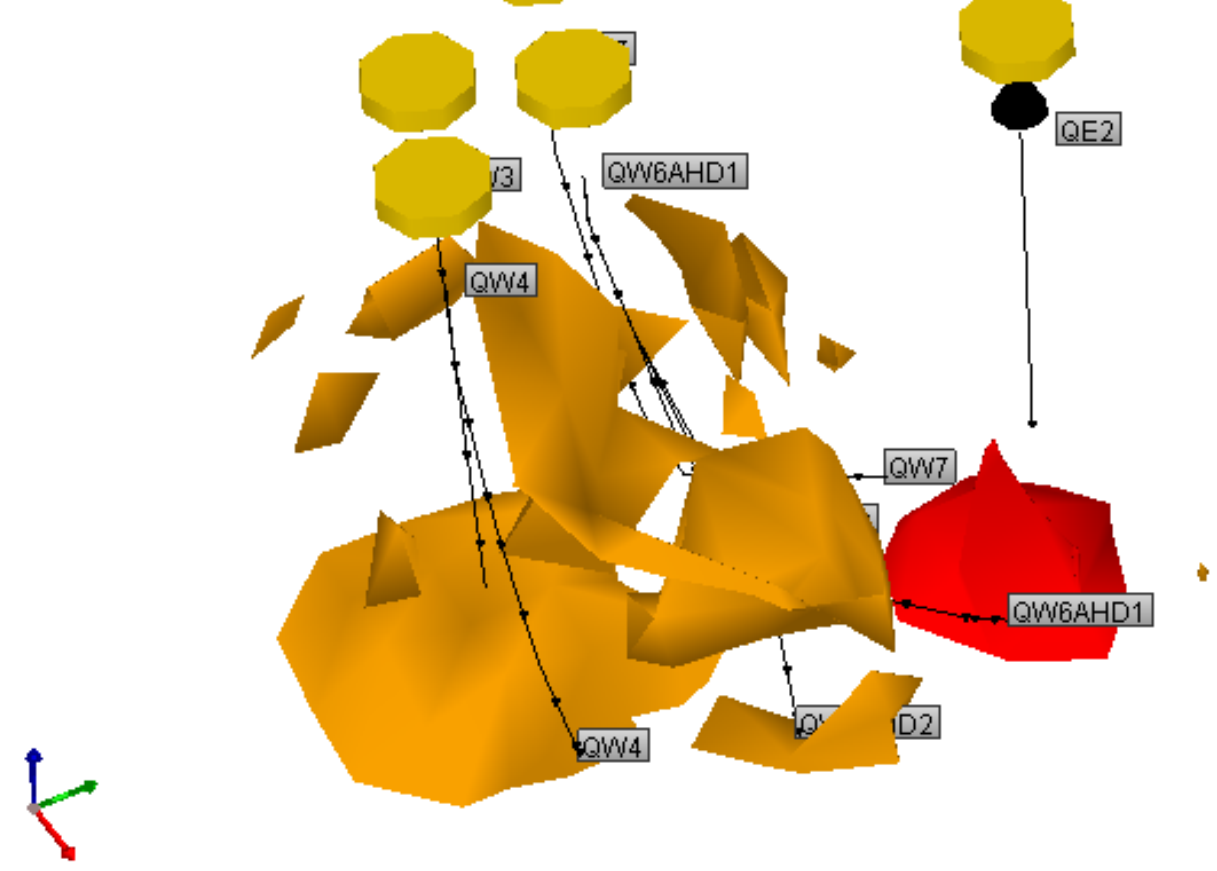

Figure 18. Isosurface of Oil Saturation at after eighteen(18) years of primary production 


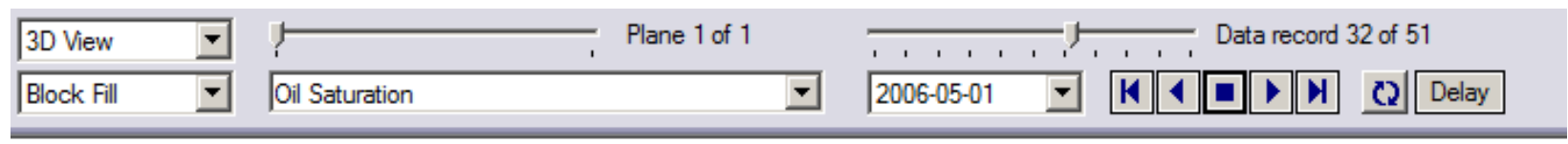

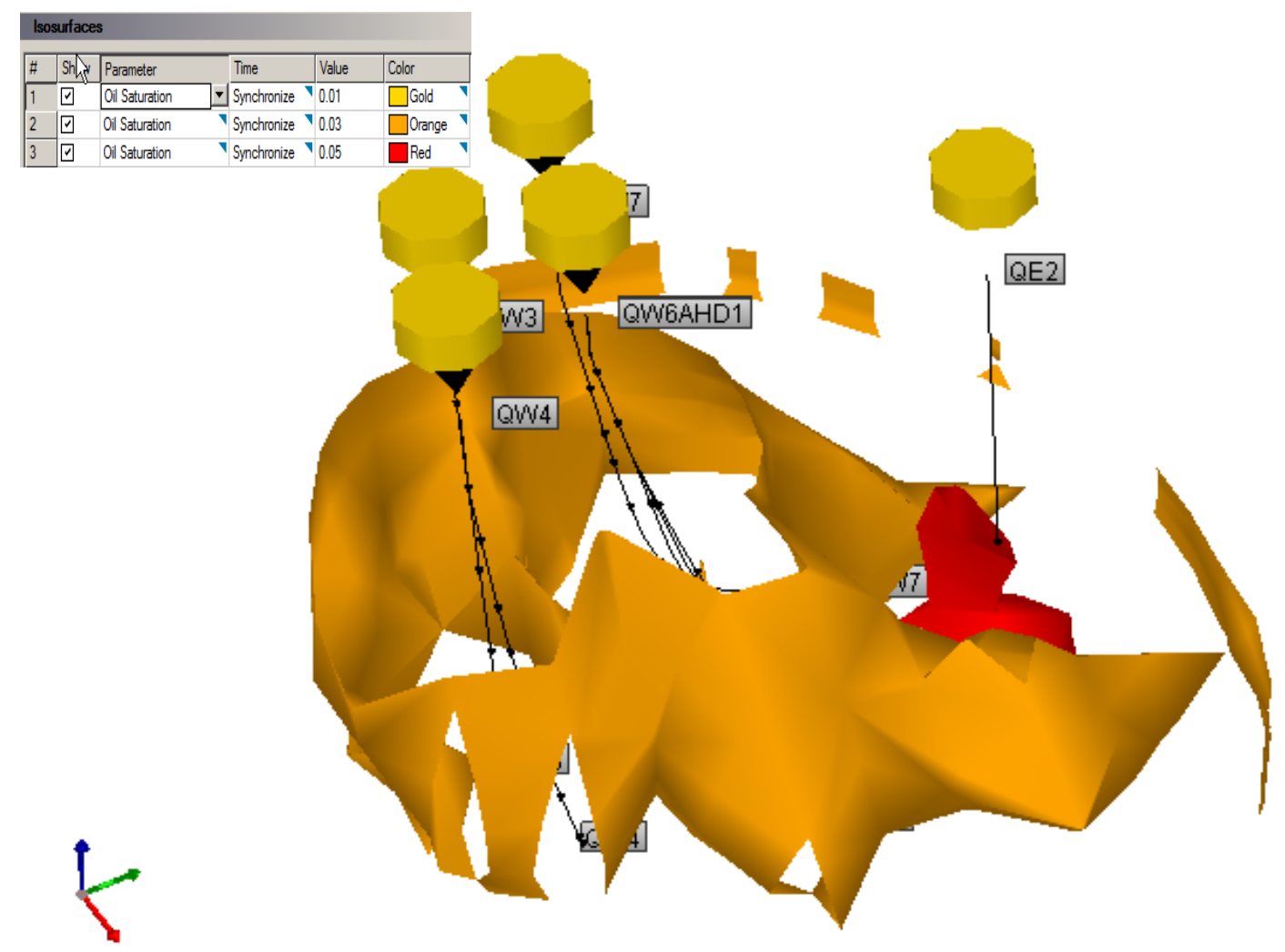

Figure 19. Isosurface of Oil Saturation at beginning of storage 


\begin{tabular}{|c|c|c|c|c|c|c|c|c|c|c|c|c|}
\hline 3D View & 7 & 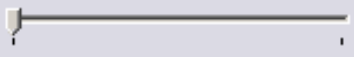 & Plane 1 of 1 & - & & & $\overline{1}$ & & a recc & & 39 of 5 & \\
\hline Block Fill & 7 & Oil Saturation & $\nabla$ & 2006-12-01 & $\nabla$ & $\mathbf{1 4}$ & 4 & $\square$ & $\nabla$ & M & C) & Delay \\
\hline
\end{tabular}

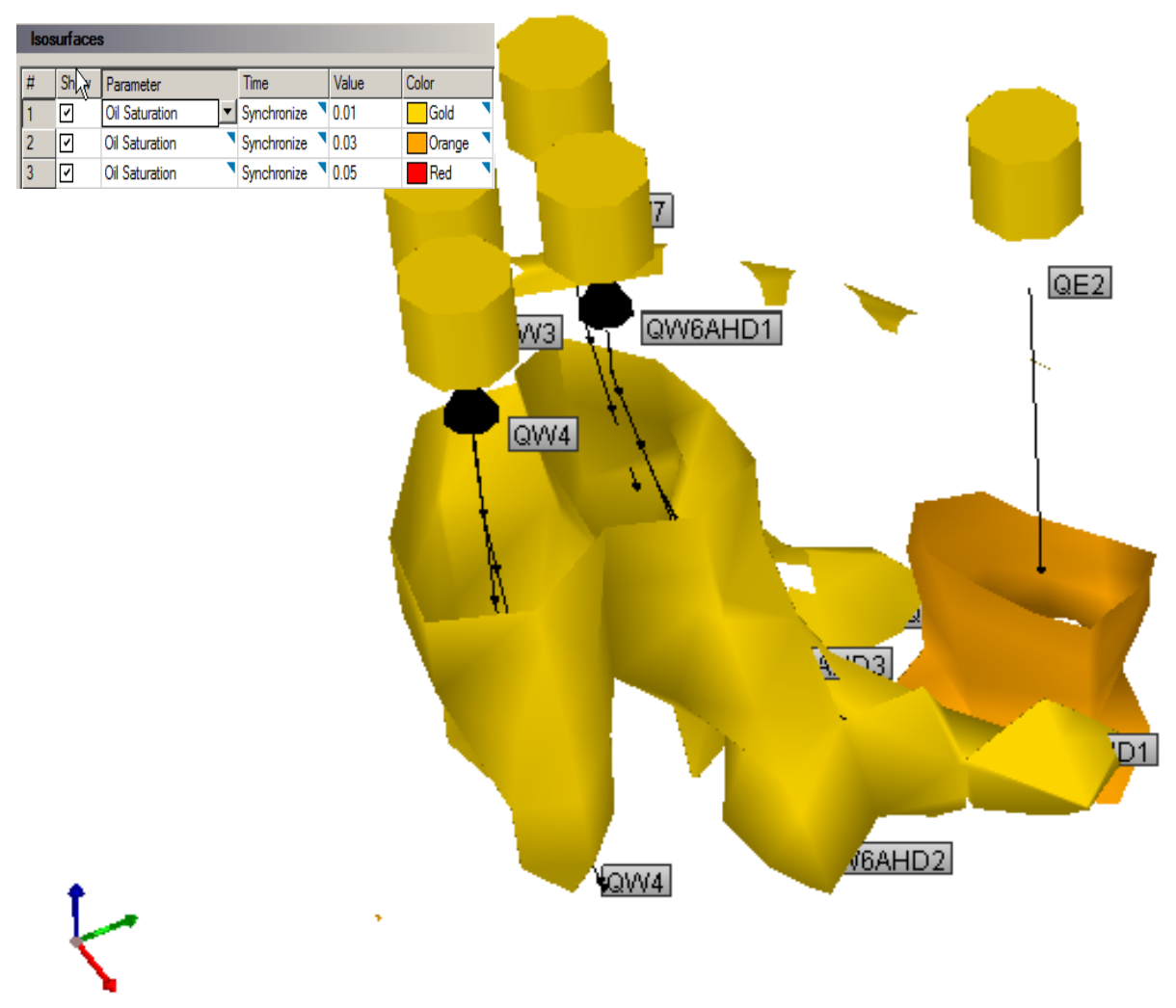

Figure 20. Isosurface of Oil Saturation at end of first storage injection cycle (note increased saturation at old production well) 


\begin{tabular}{|c|c|c|c|c|c|c|c|c|c|c|c|c|}
\hline 3D View & $\nabla$ & ? & Plane 1 of 1 & 1,1 & & - & $\overline{1}$ & Dat & rec & cord & 13 of 5 & \\
\hline Block Fill & $\nabla$ & Oil Saturation & $\nabla$ & 2007-04-01 & $\nabla$ & $\mathbf{K}$ & 4 & $\square$ & $\nabla$ & $\mathbf{Y}$ & C) & Delay \\
\hline
\end{tabular}

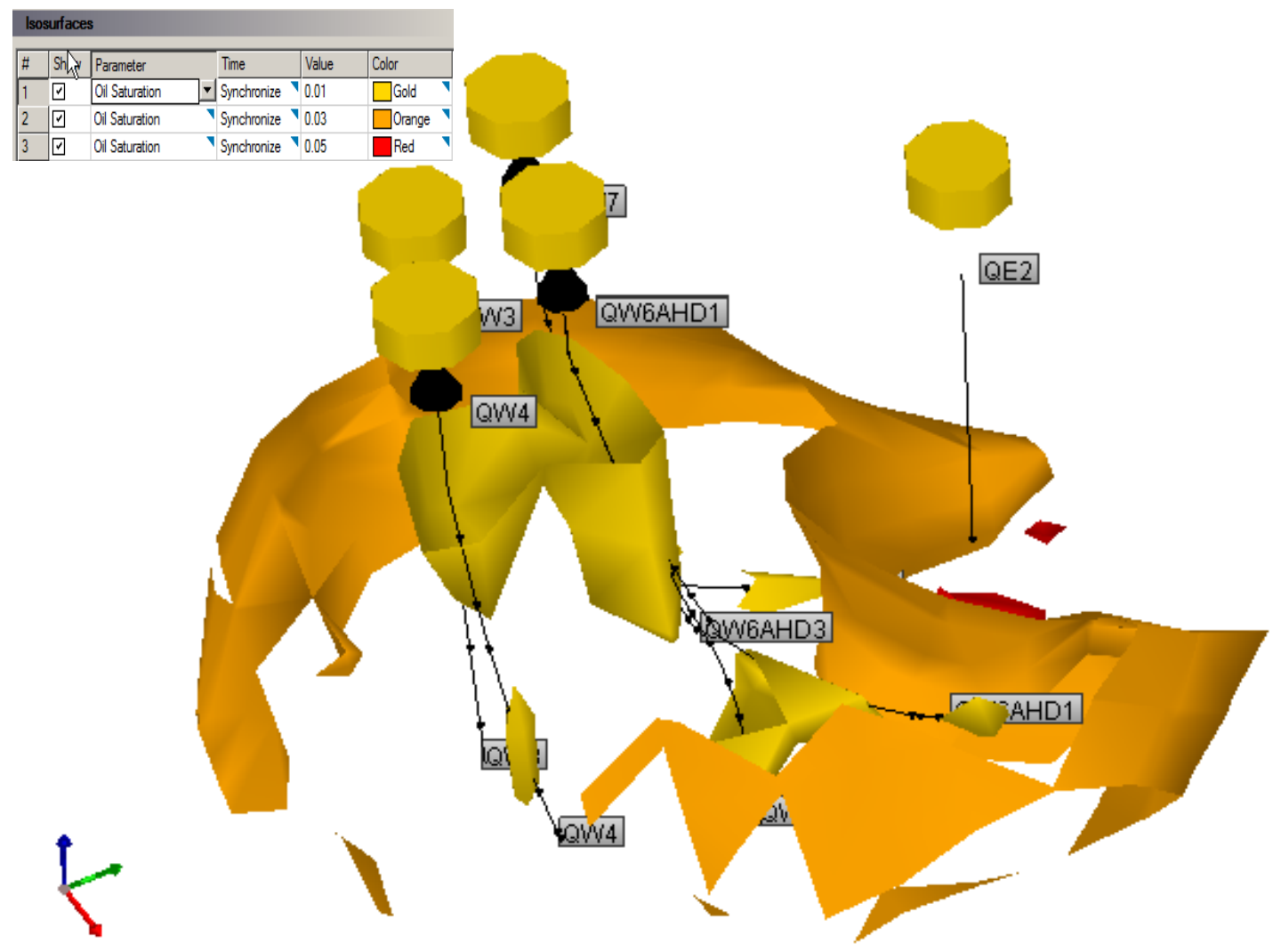

Figure 21. Isosurface of Oil Saturation at end of first storage withdrawal cycle (note increased saturation at old production well) 


\begin{tabular}{|c|c|c|c|c|c|c|c|c|c|c|}
\hline 3D View & $\nabla$ & ! & Plane 1 of 1 & 1,1 & 1 & -) Dat & ta reco & cord 51 & 1 of 51 & \\
\hline Block Fill & $\nabla$ & Oil Saturation & $\nabla$ & 2007-12-01 & $\mathbf{H}$ & 10 & 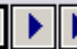 & M & $Q \mid$ & Delay \\
\hline
\end{tabular}

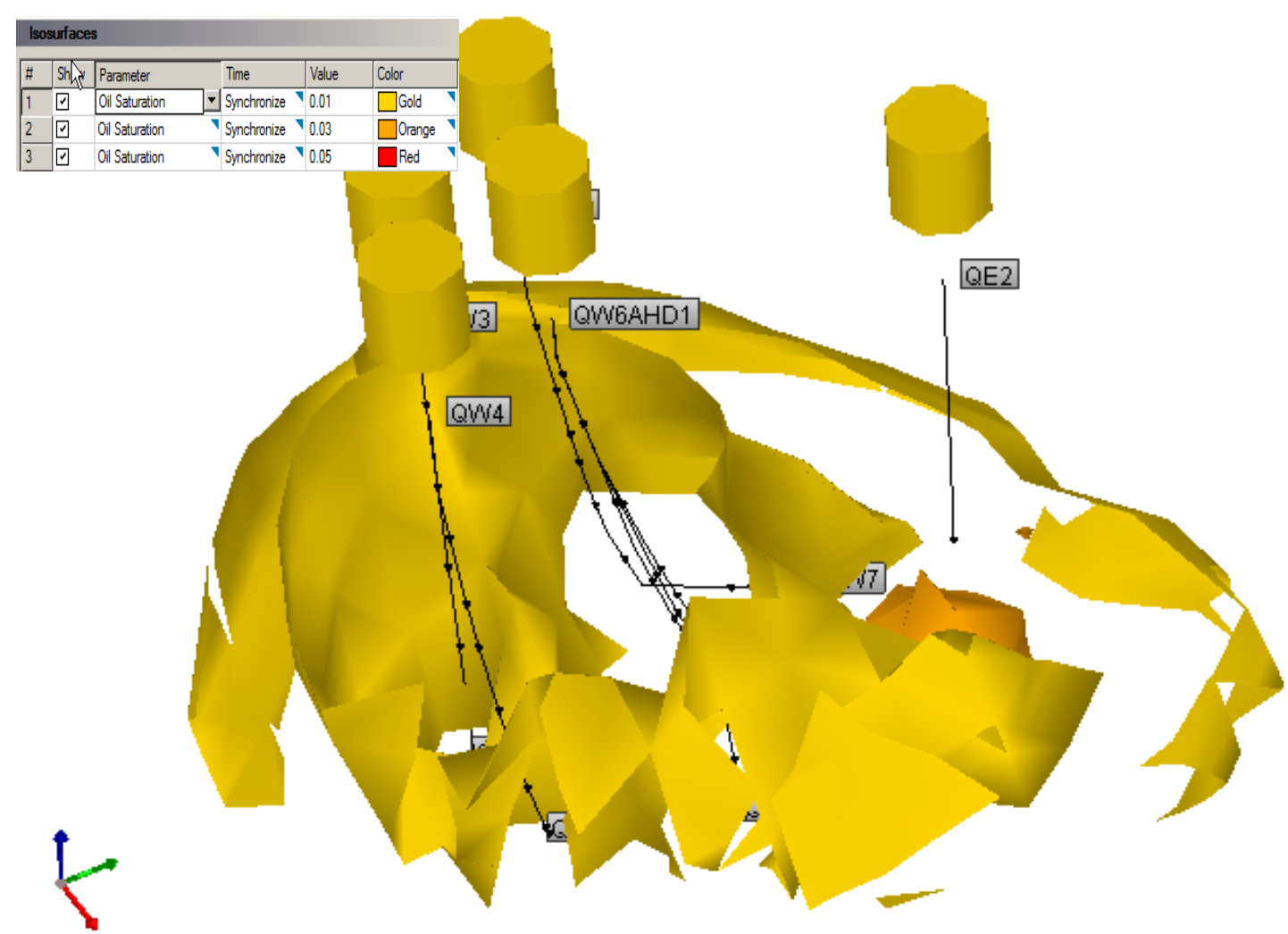

Figure 22. Isosurface of Oil Saturation at end of second storage injection cycle (note increased saturation at old production well) 
Constant Composition Expansion(CCE)

1. Before Regression
a. Relative Volume
b. Oil Viscosity
c. Liquid Volume \%Cell Vol.
d. Gas Z factor
e. Oil Compressibility
f. Gas Density

2. After Regression
a. Relative Volume
b. Oil Viscosity
c. Liquid Volume \%Cell Vol.
d. Gas Z factor
e. Oil Compressibility
f. Gas Density

1. Summary

a. Relative Volume

\section{Constant Volume Depletion(CVD)}

1. Before Regression
a. Gas Compressibility Factor
b. Liquid Volume \% Original Vol.

2. After Regression

a. Gas Compressibility Factor

b. Liquid Volume \% Original Vol.

\section{Summary}
a. Gas Compressibility Factor
b. Liquid Volume \% Original Vol.

\section{PT Diagrams}




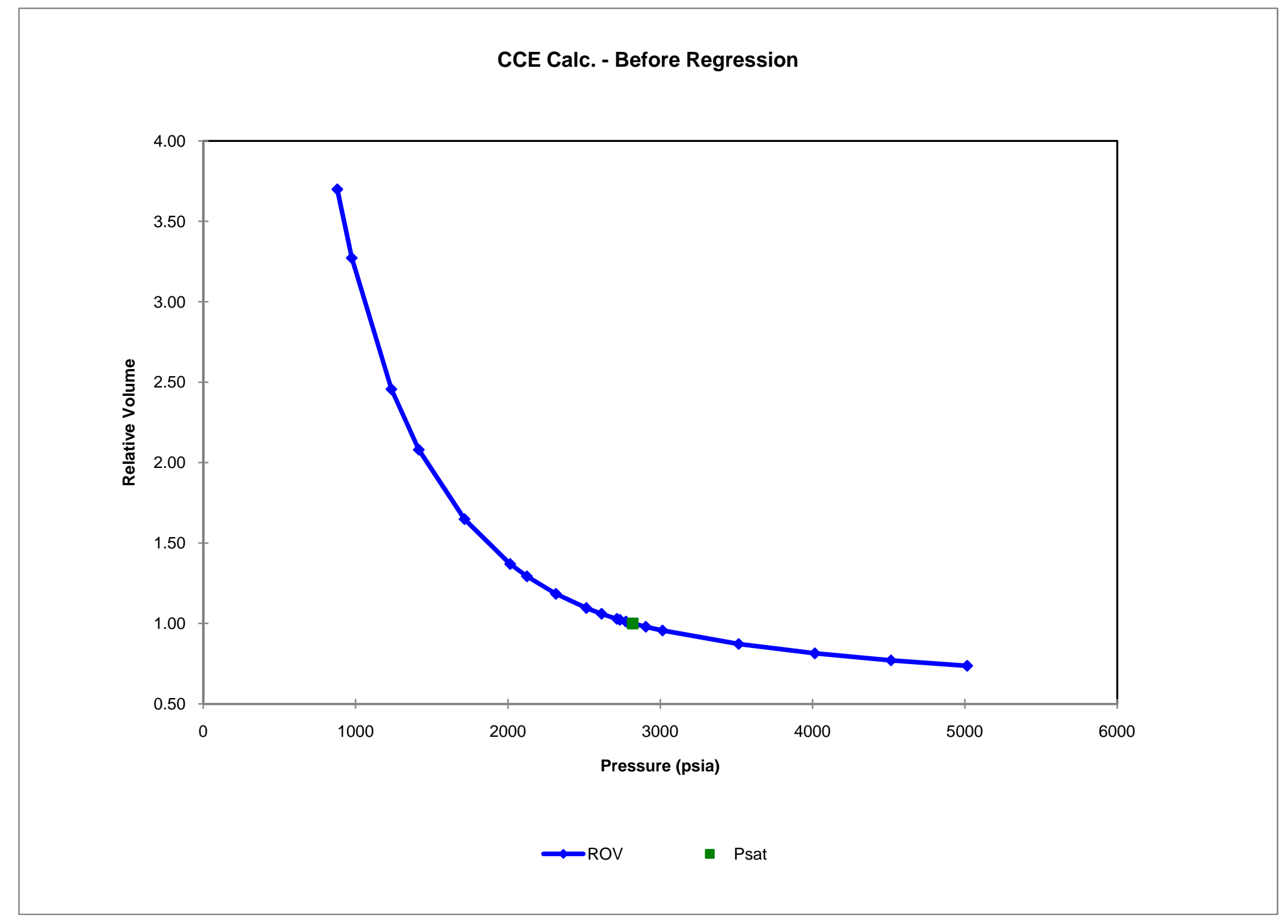




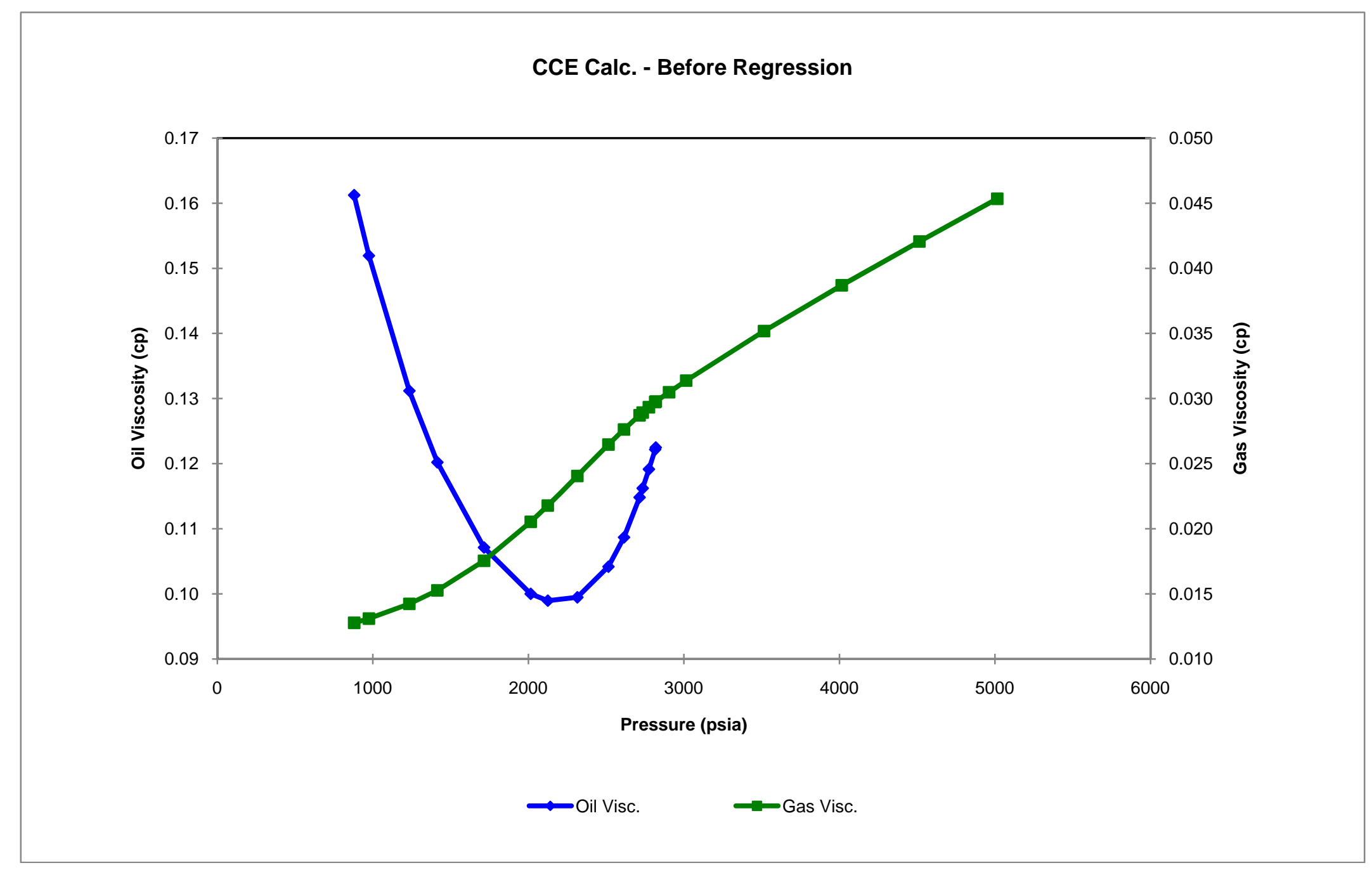




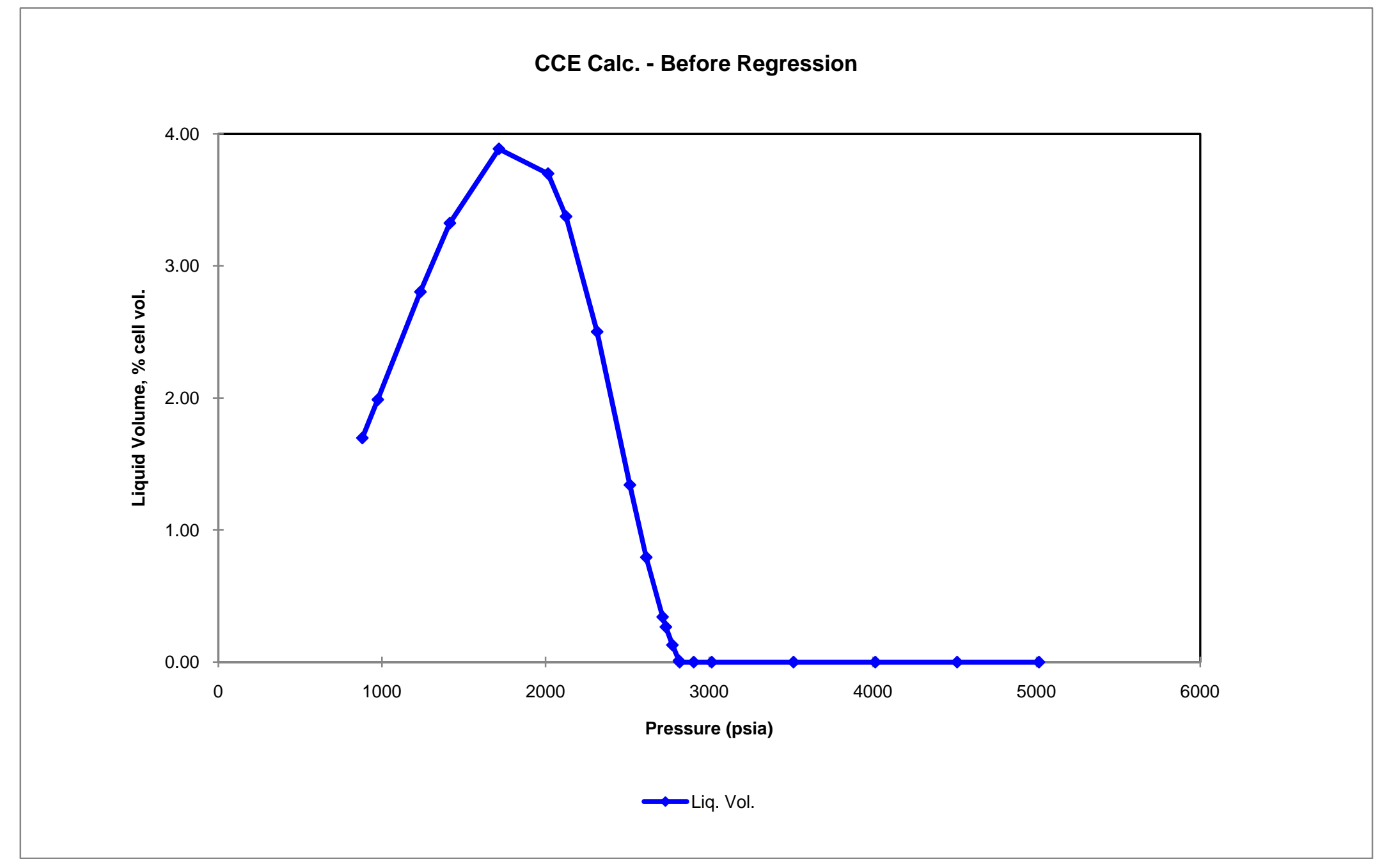




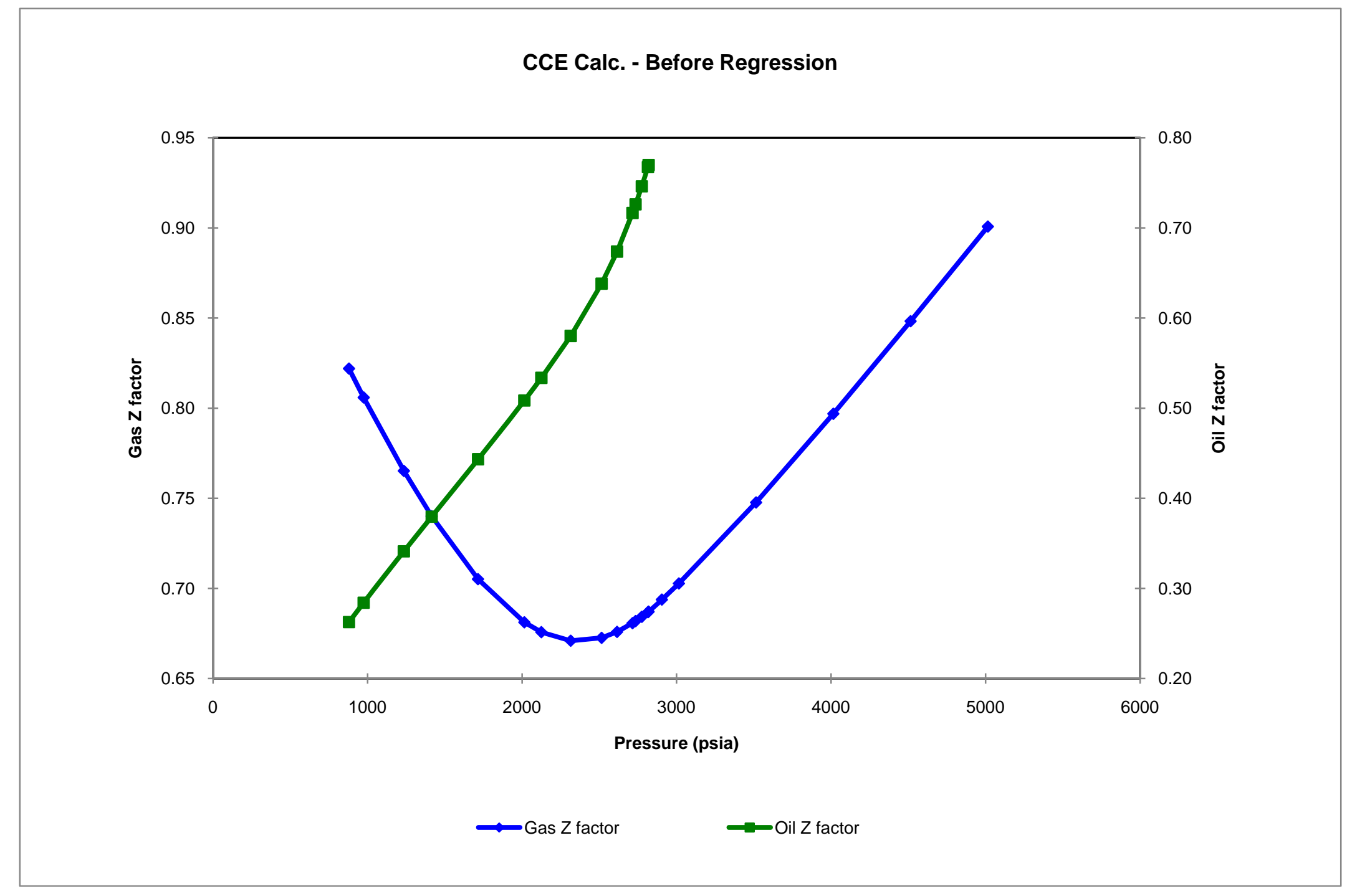




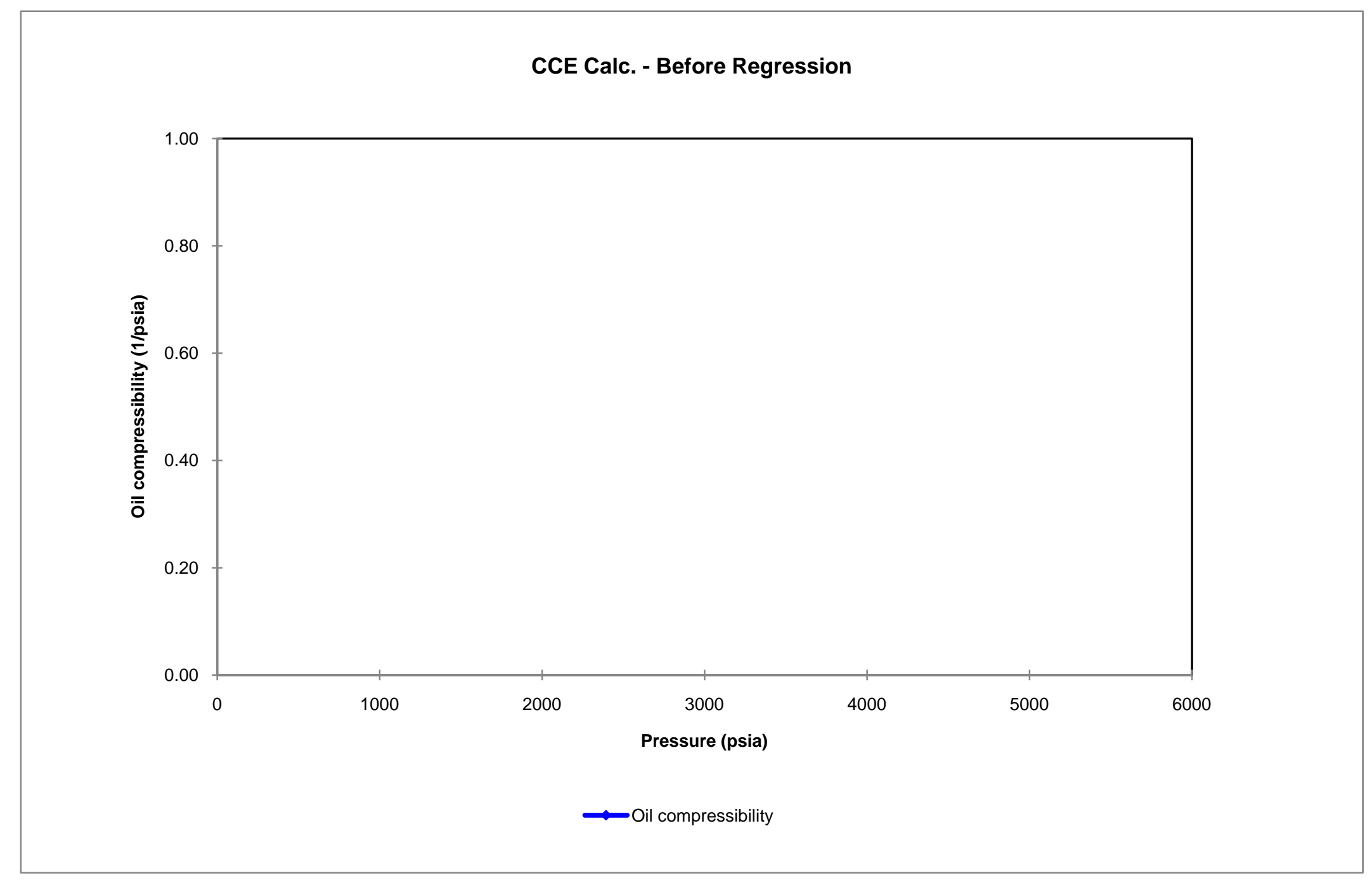




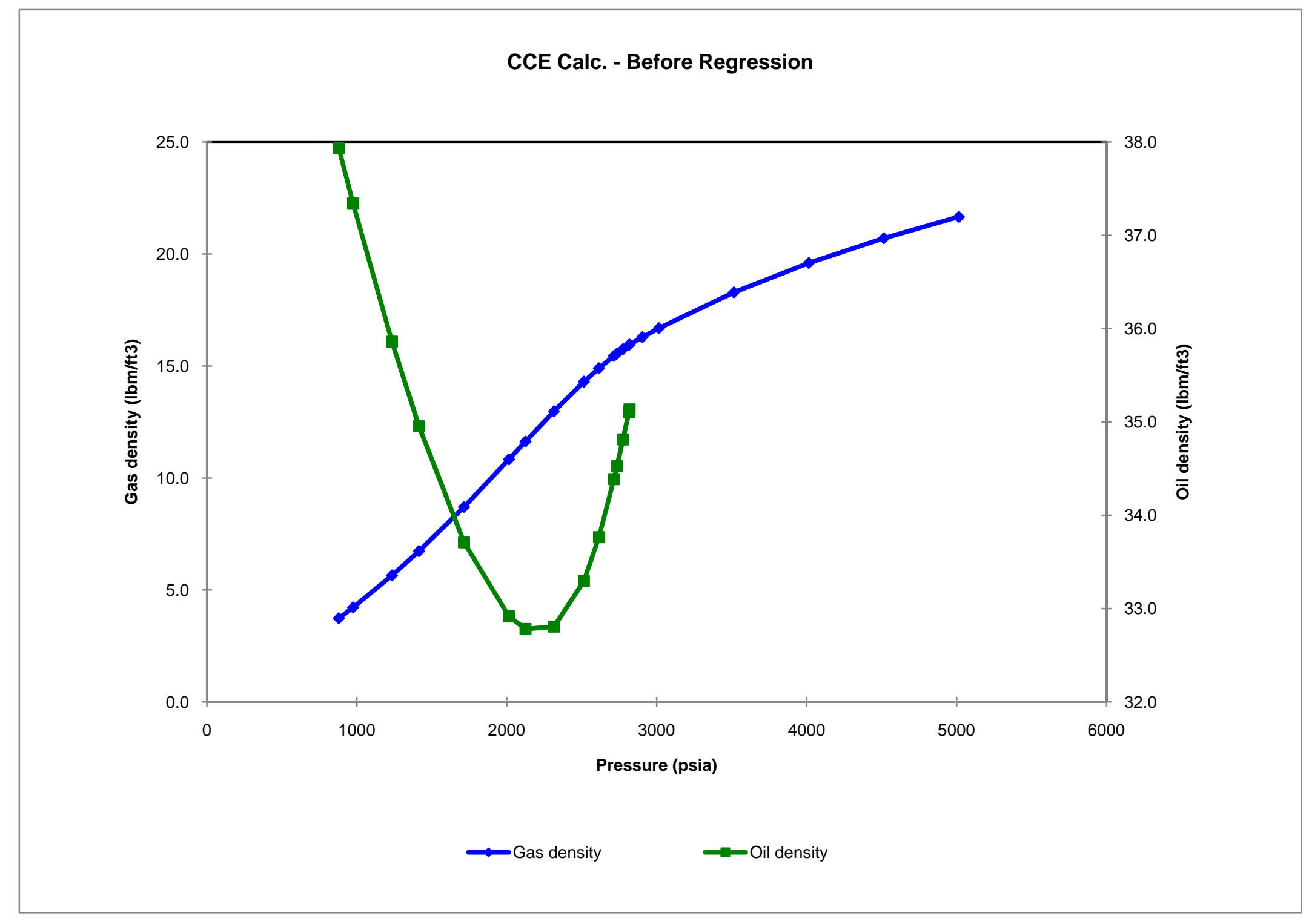




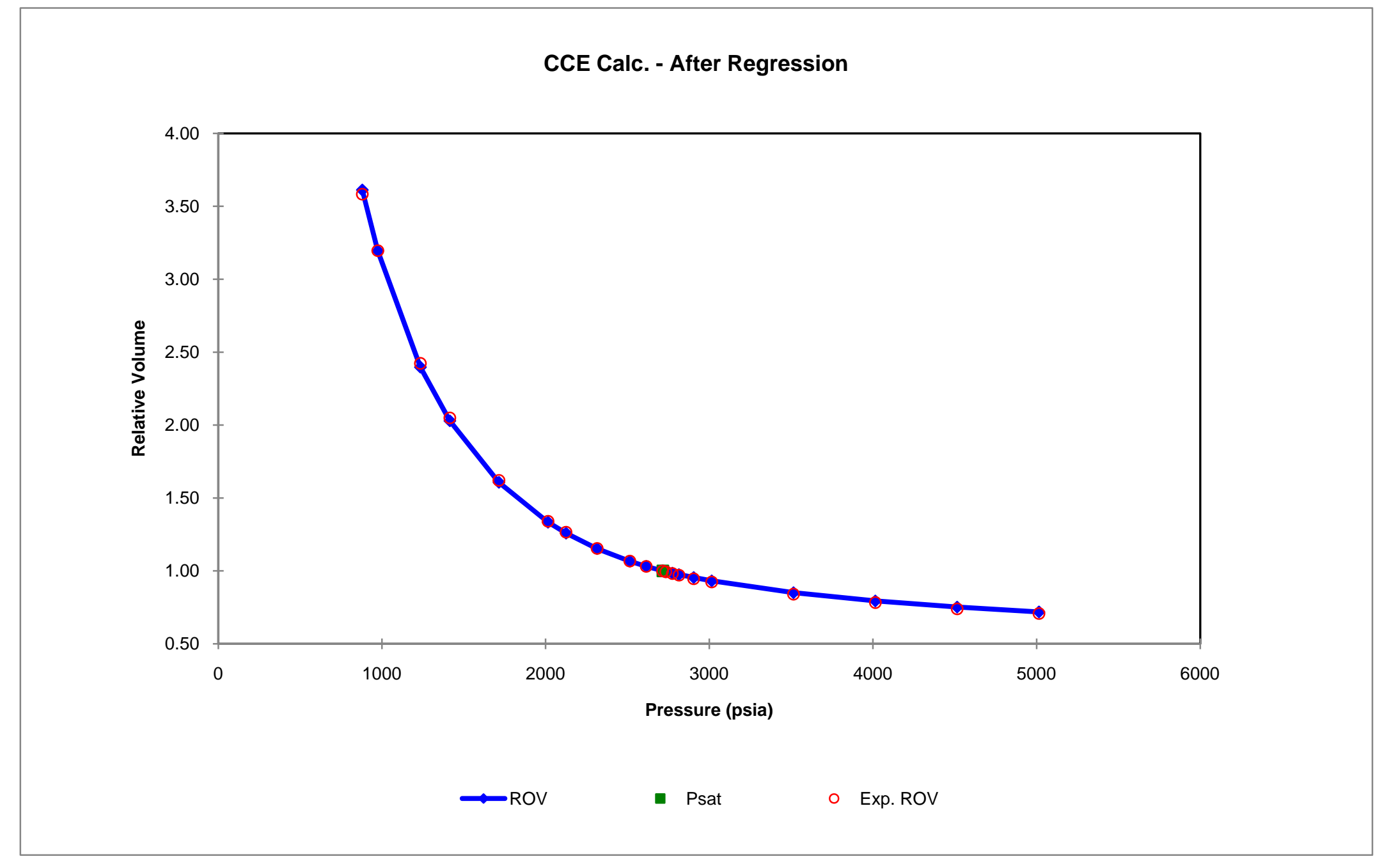




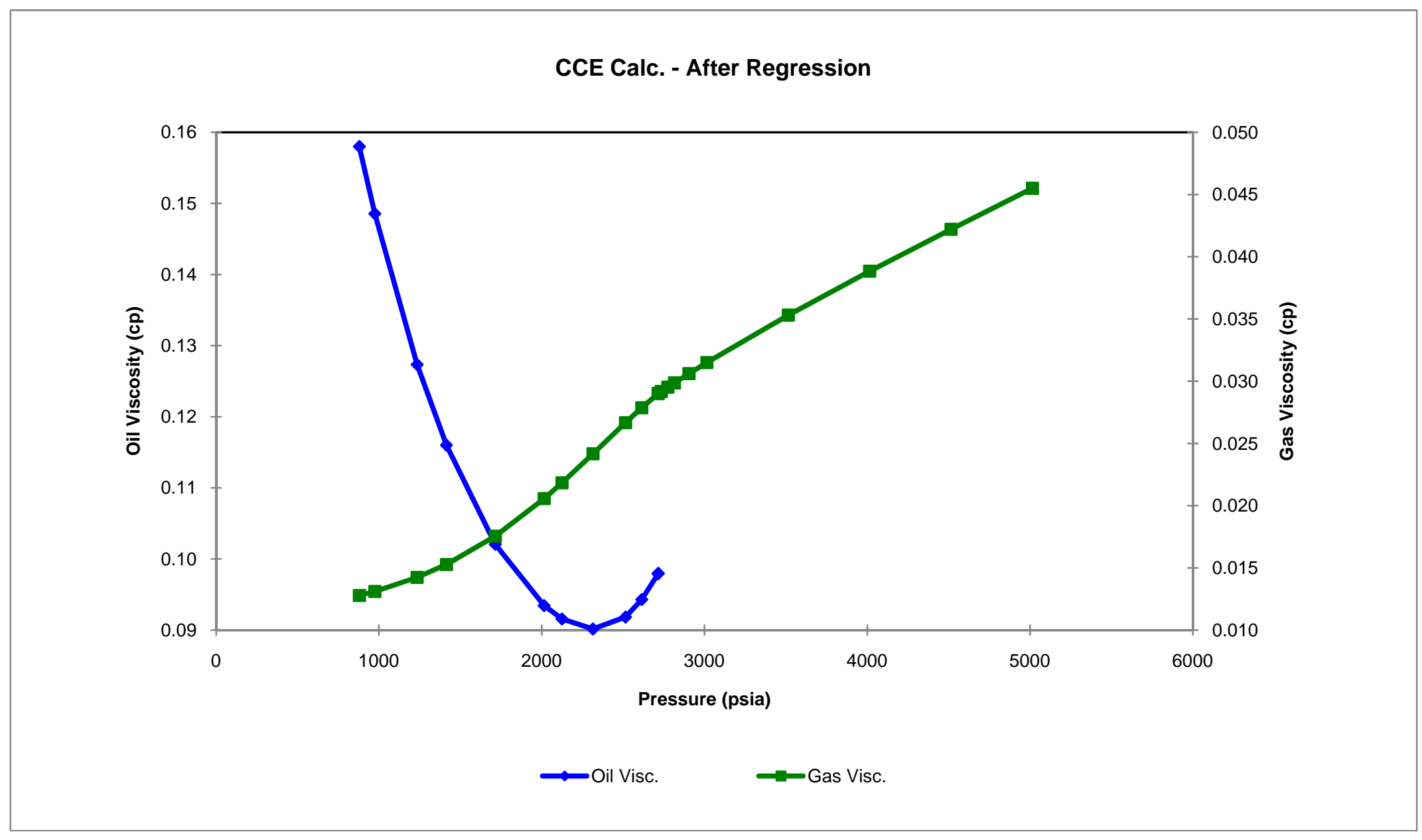




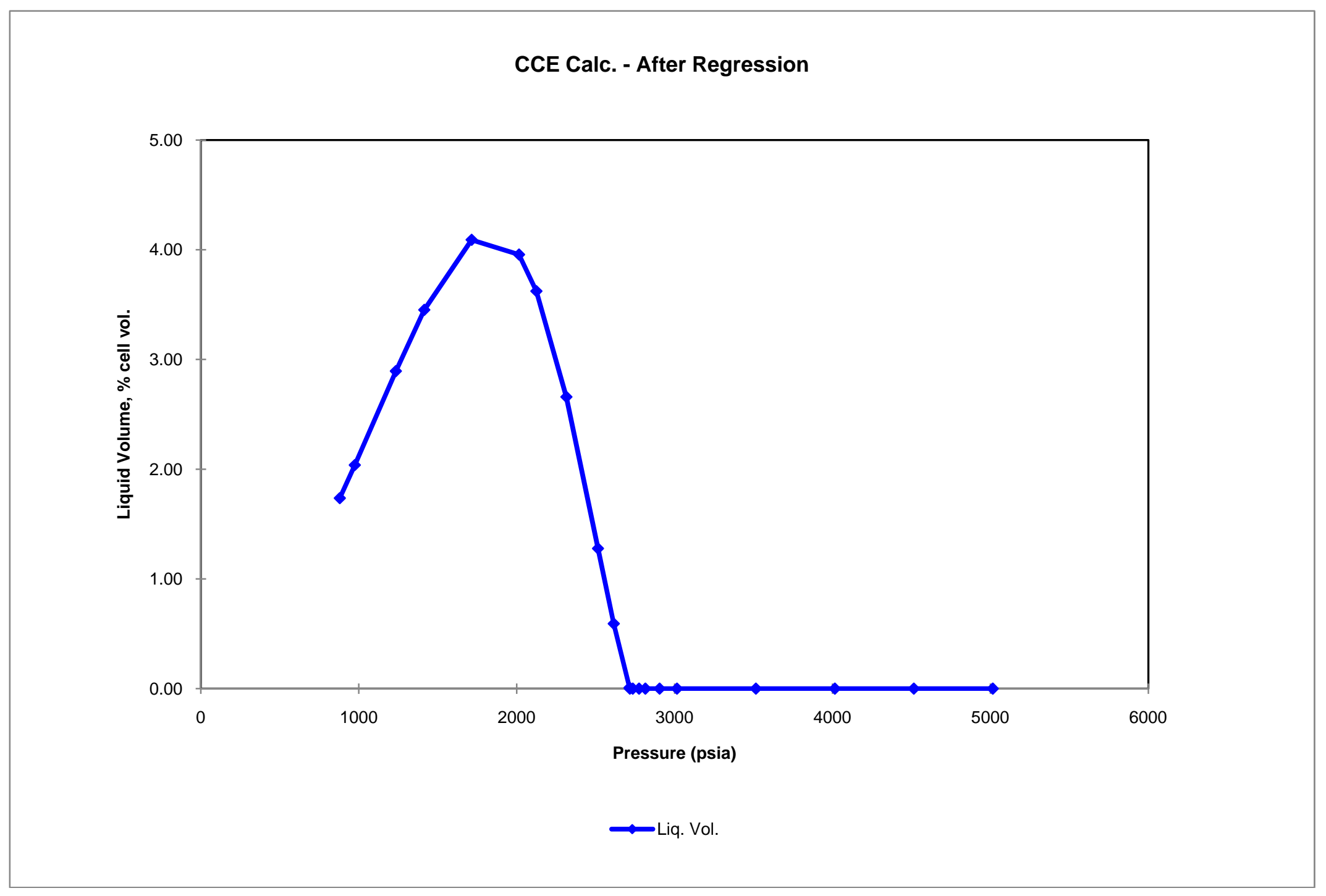

162 


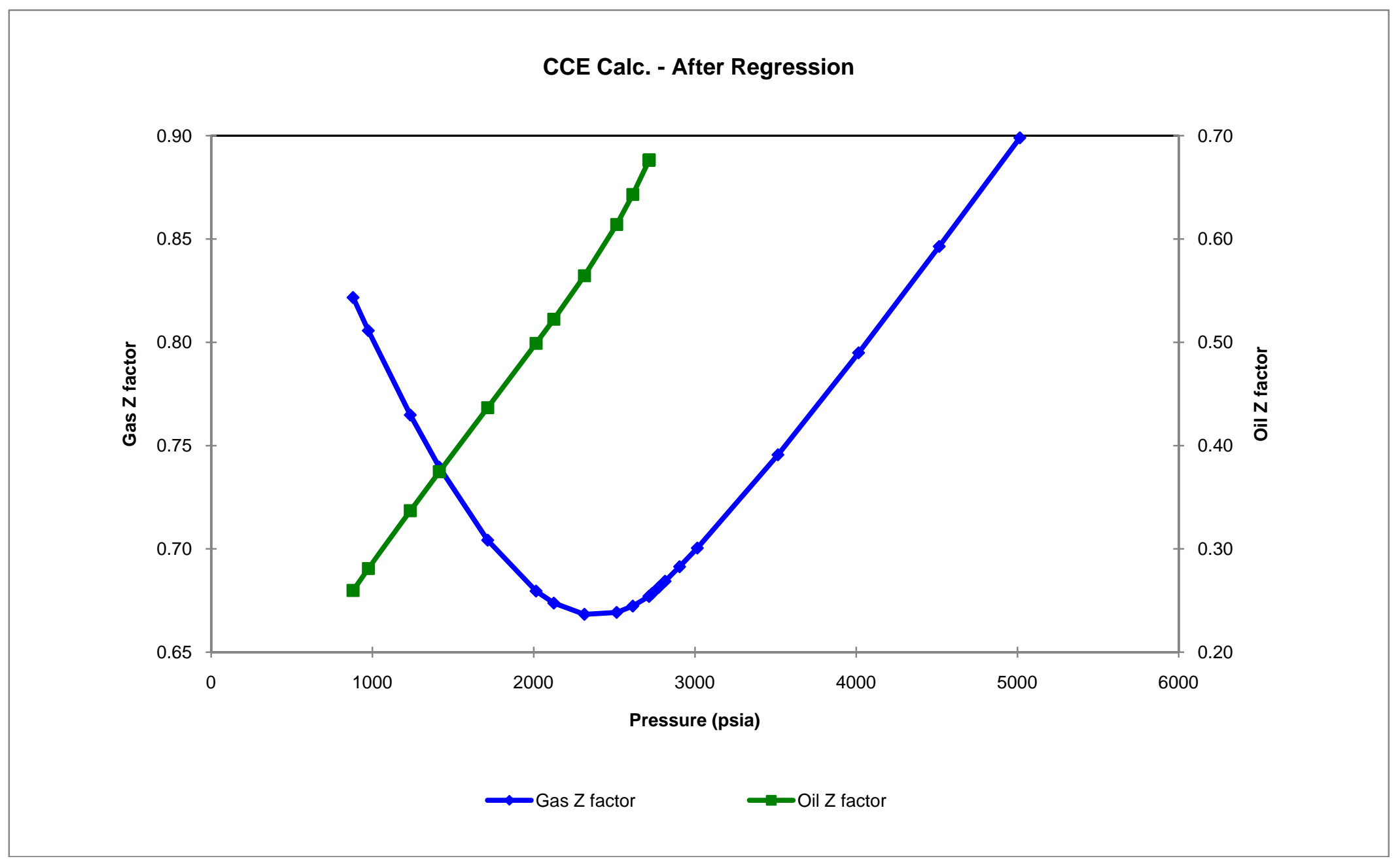




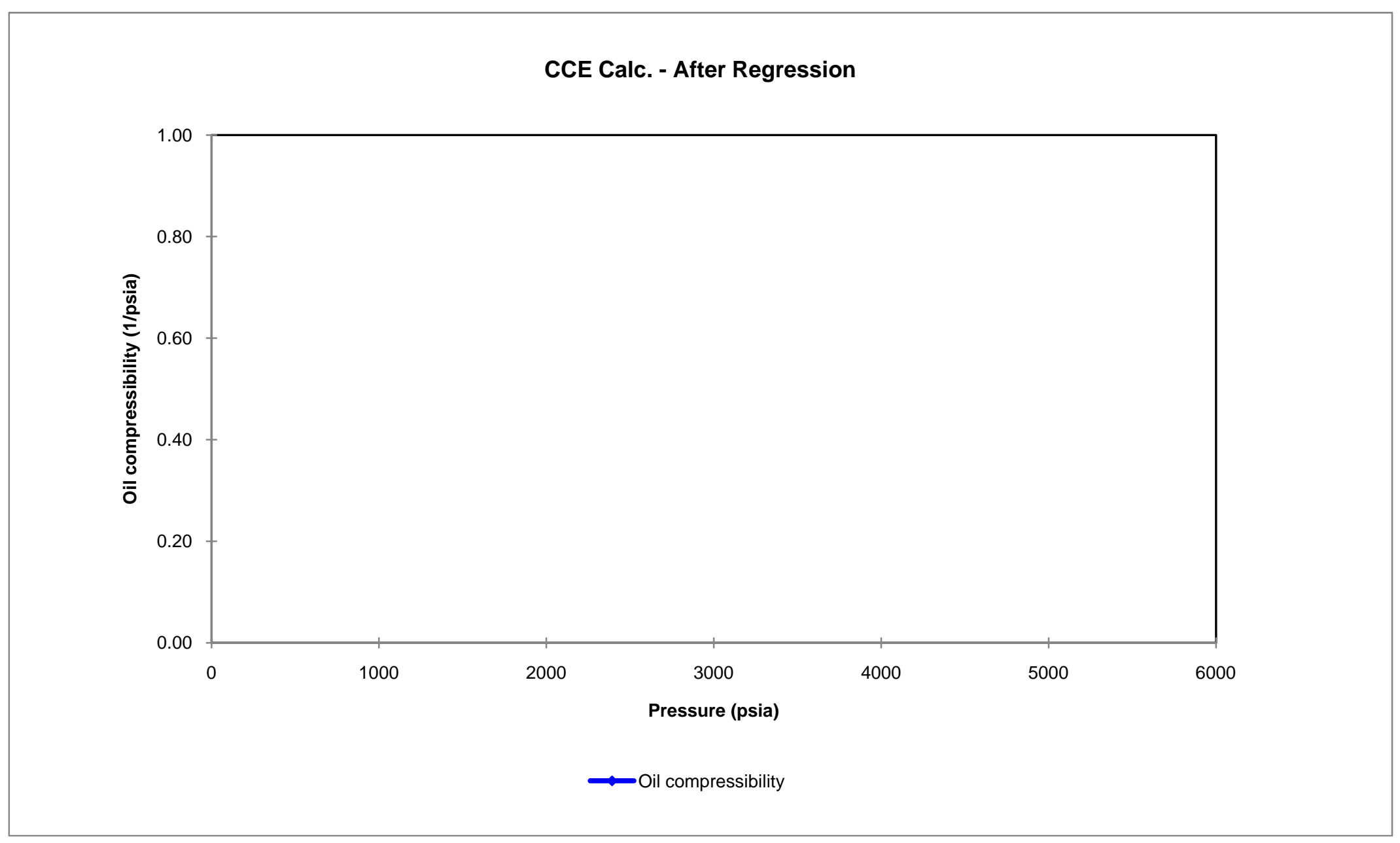




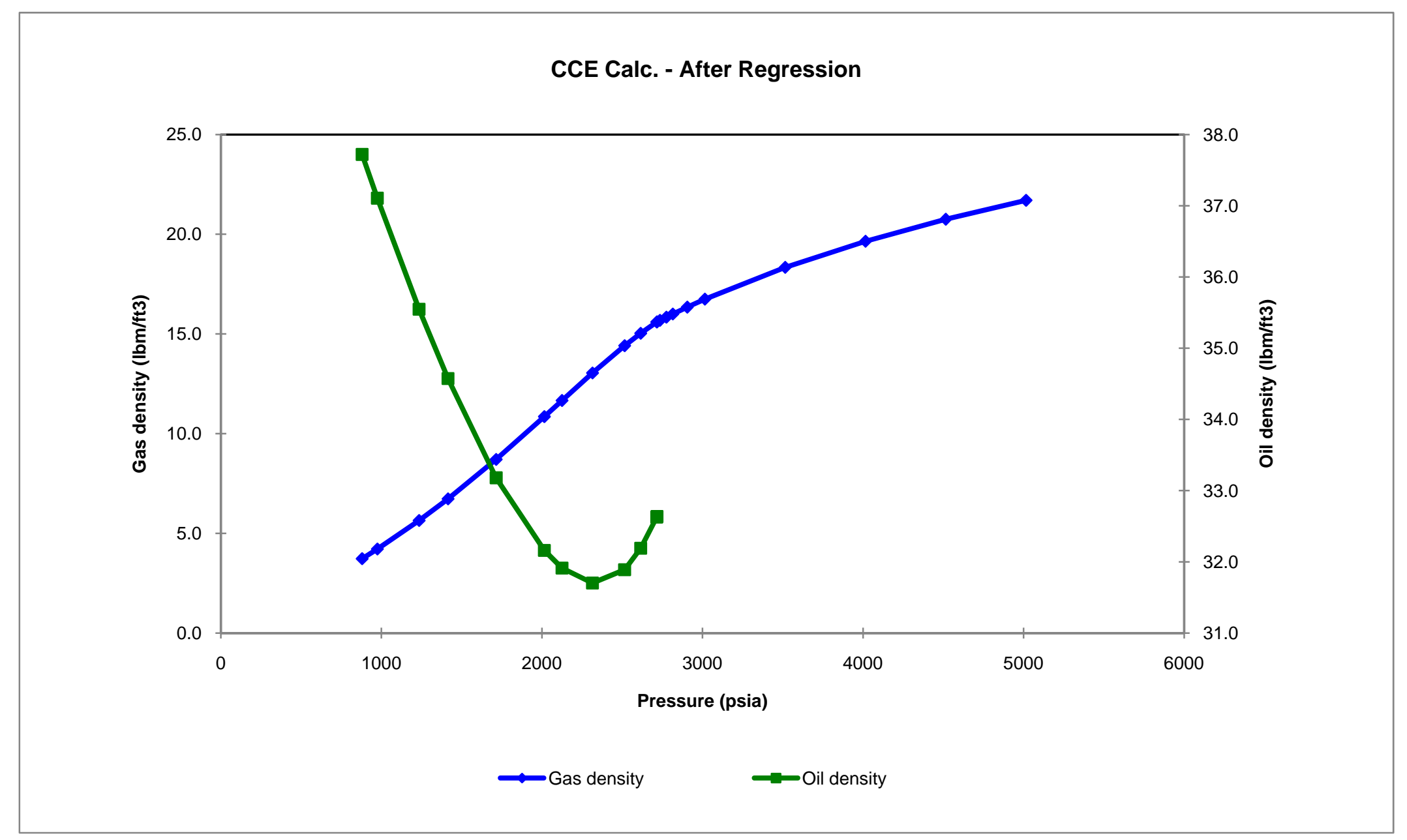




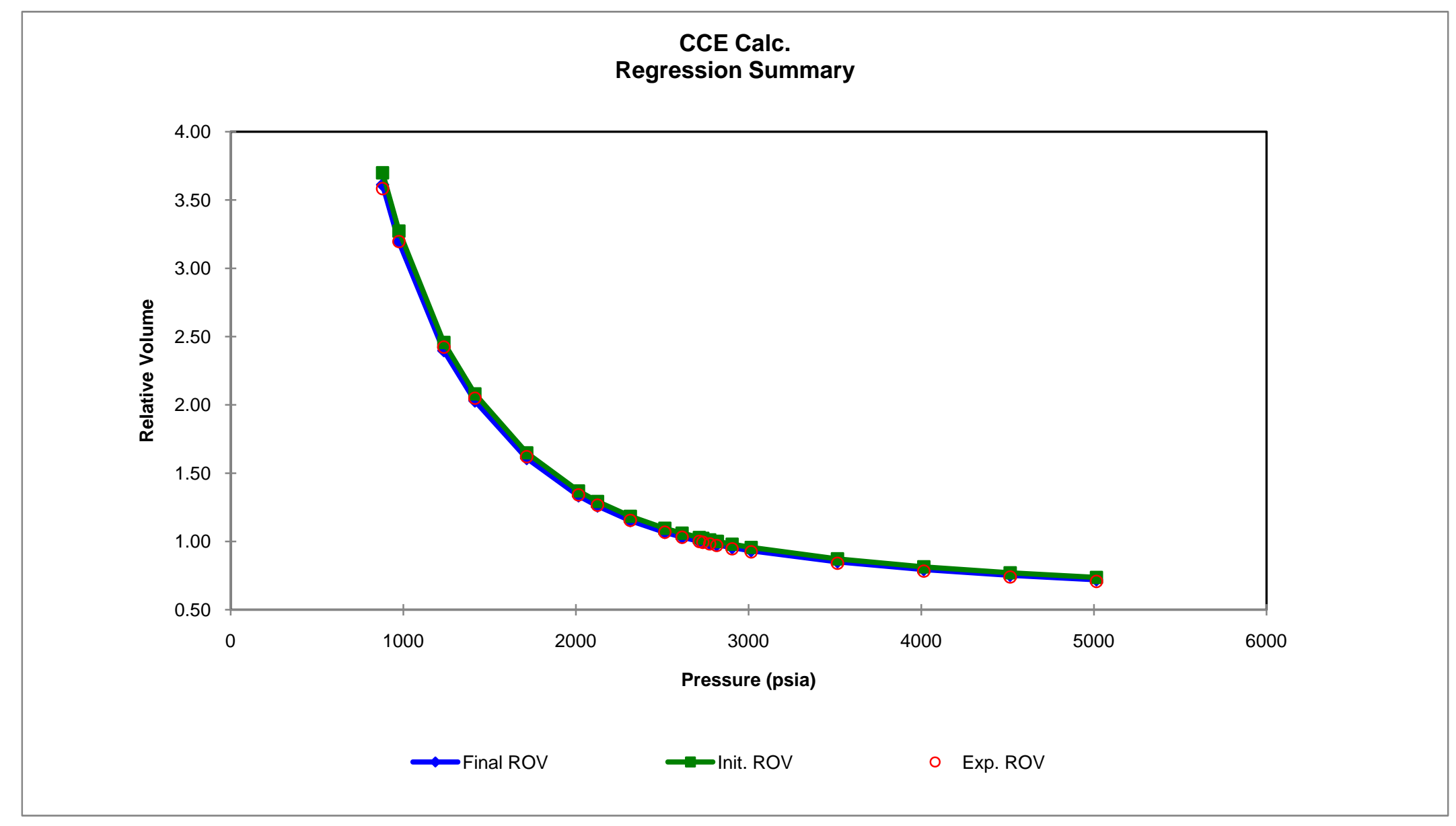




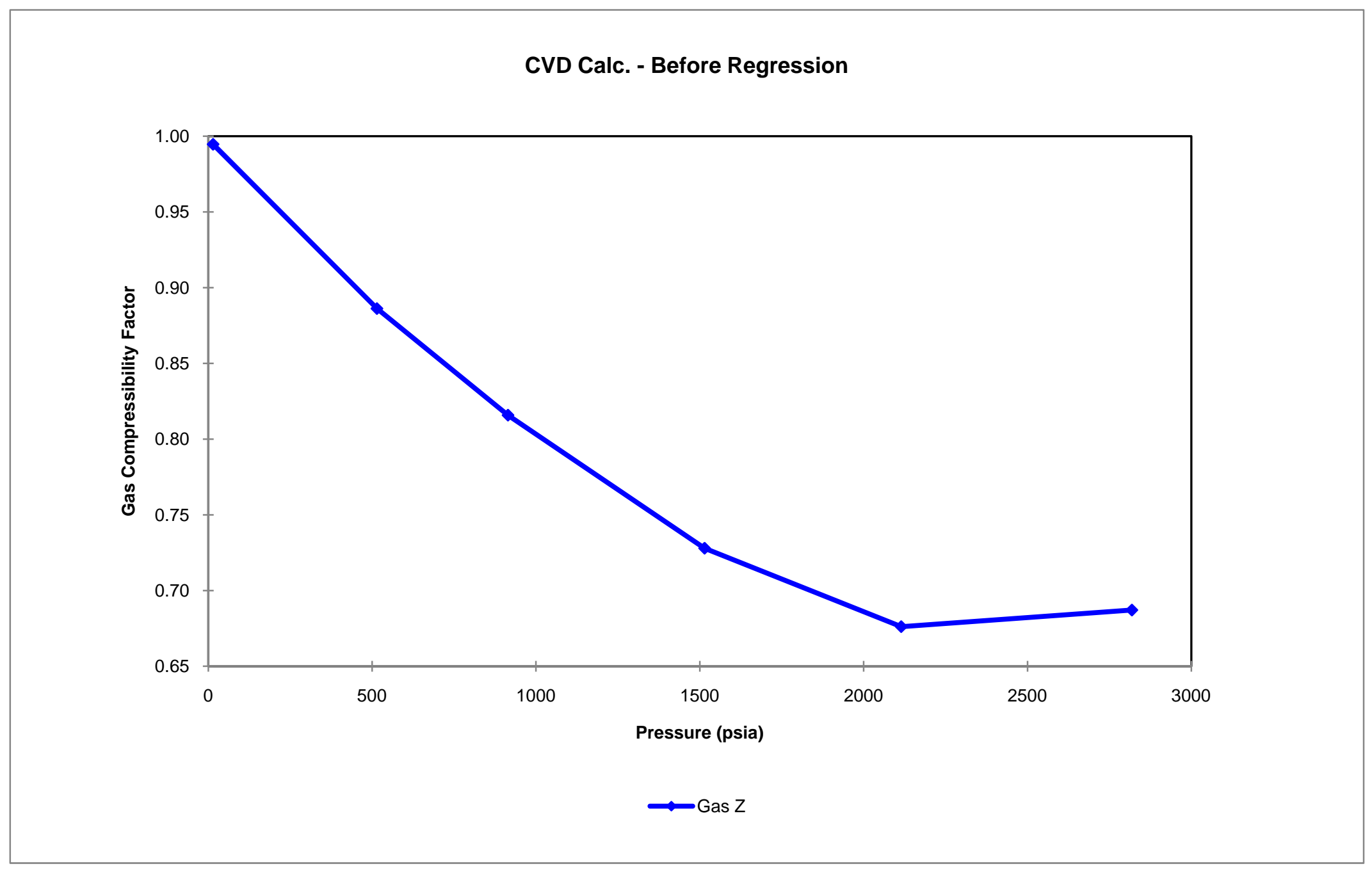




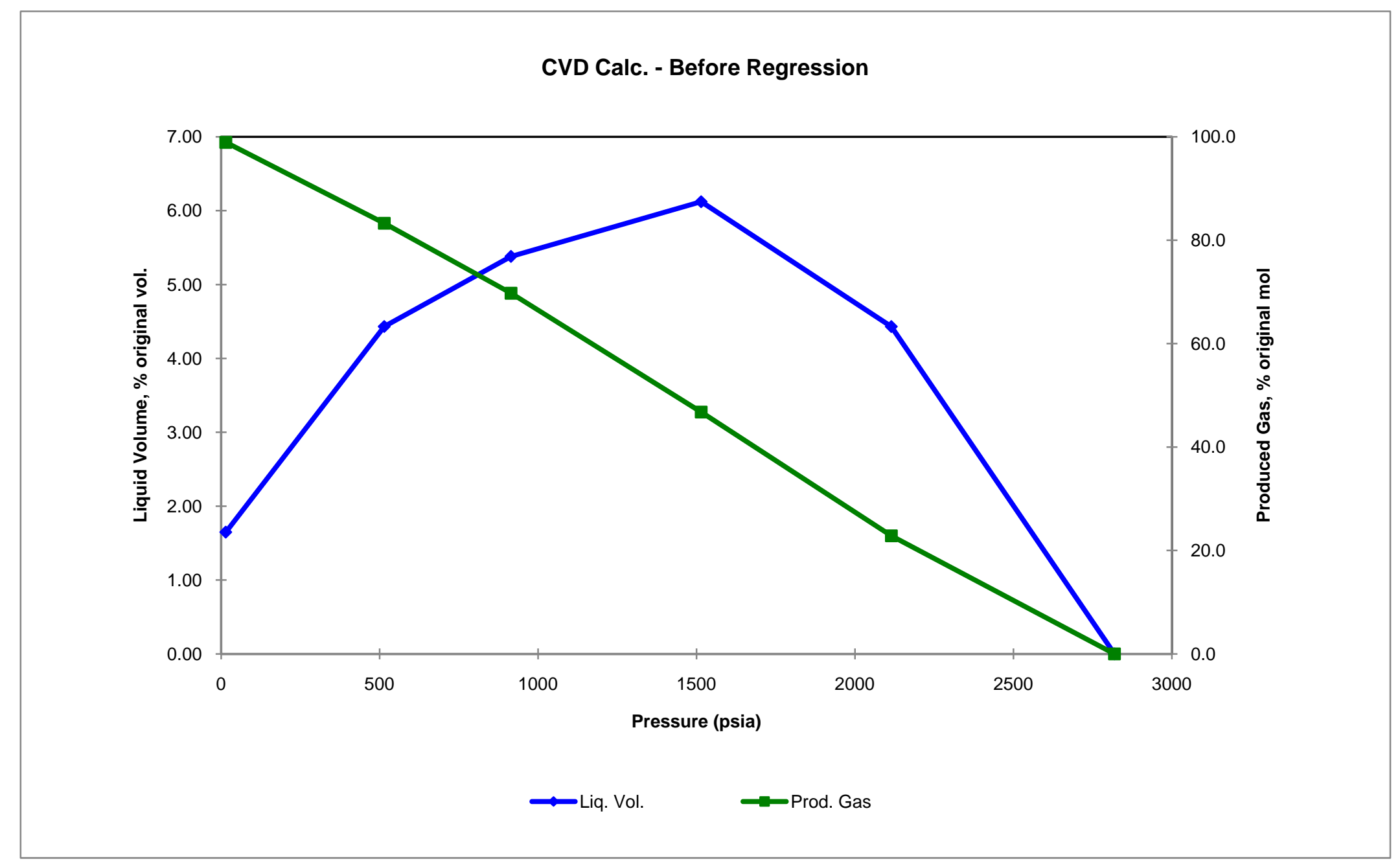




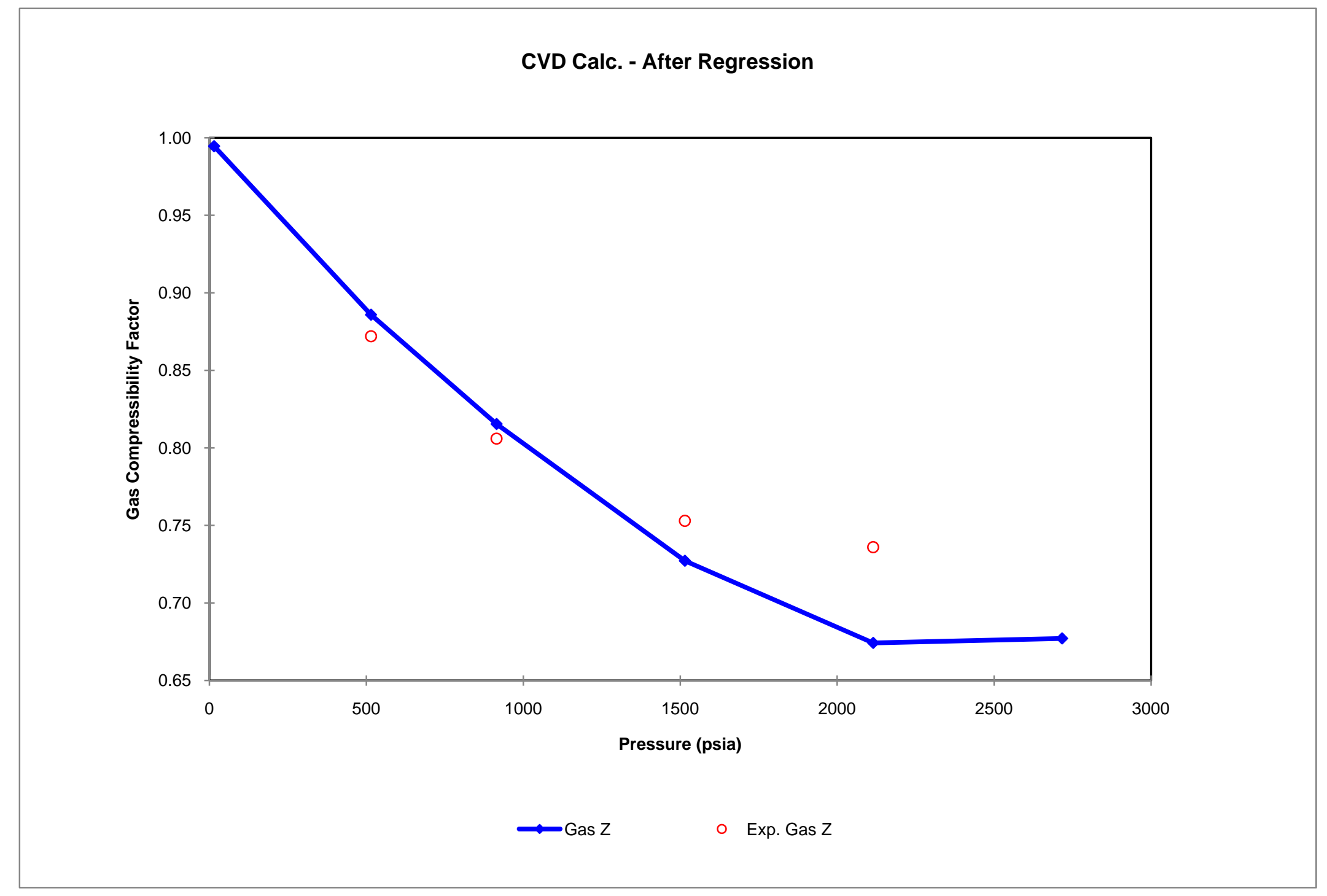




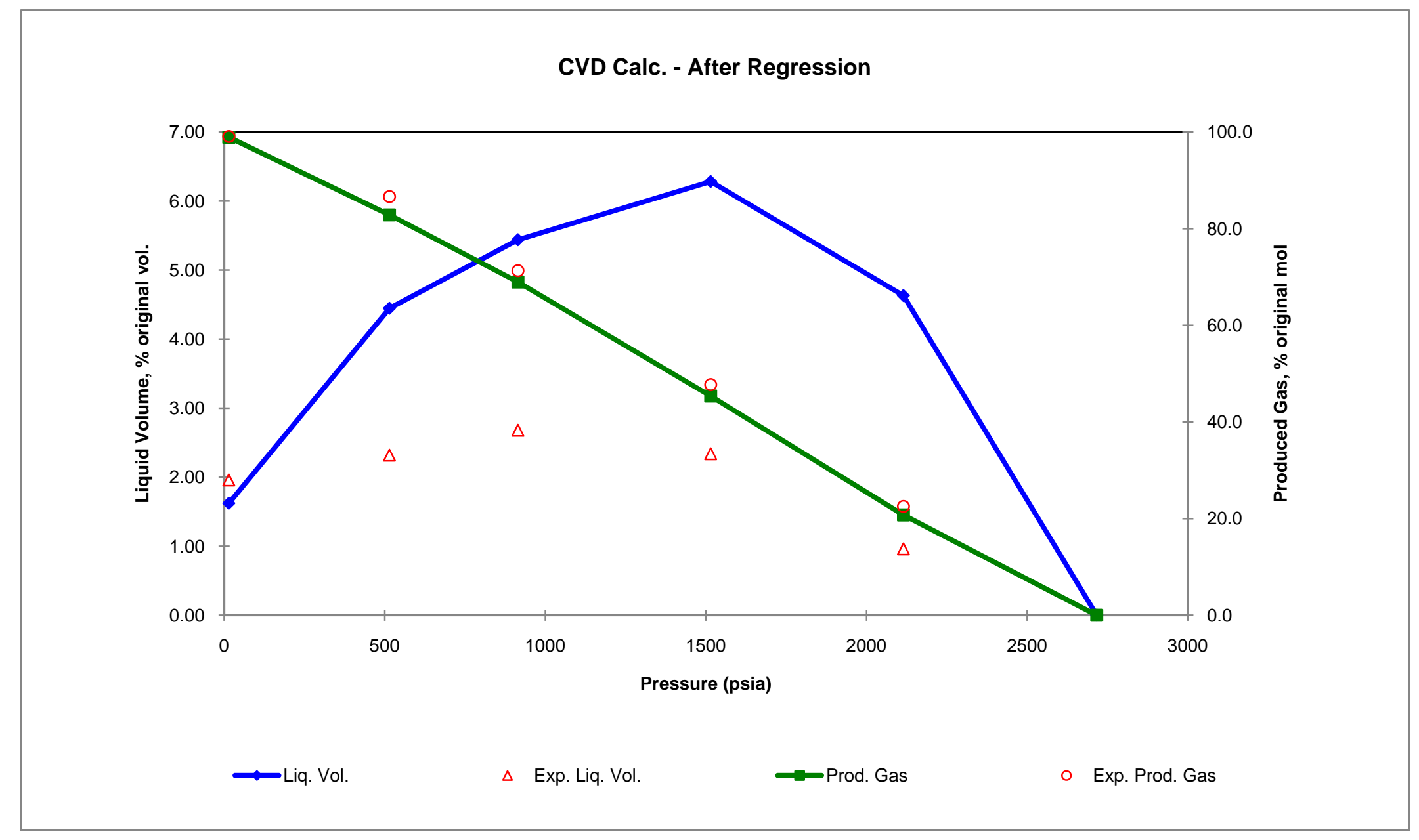




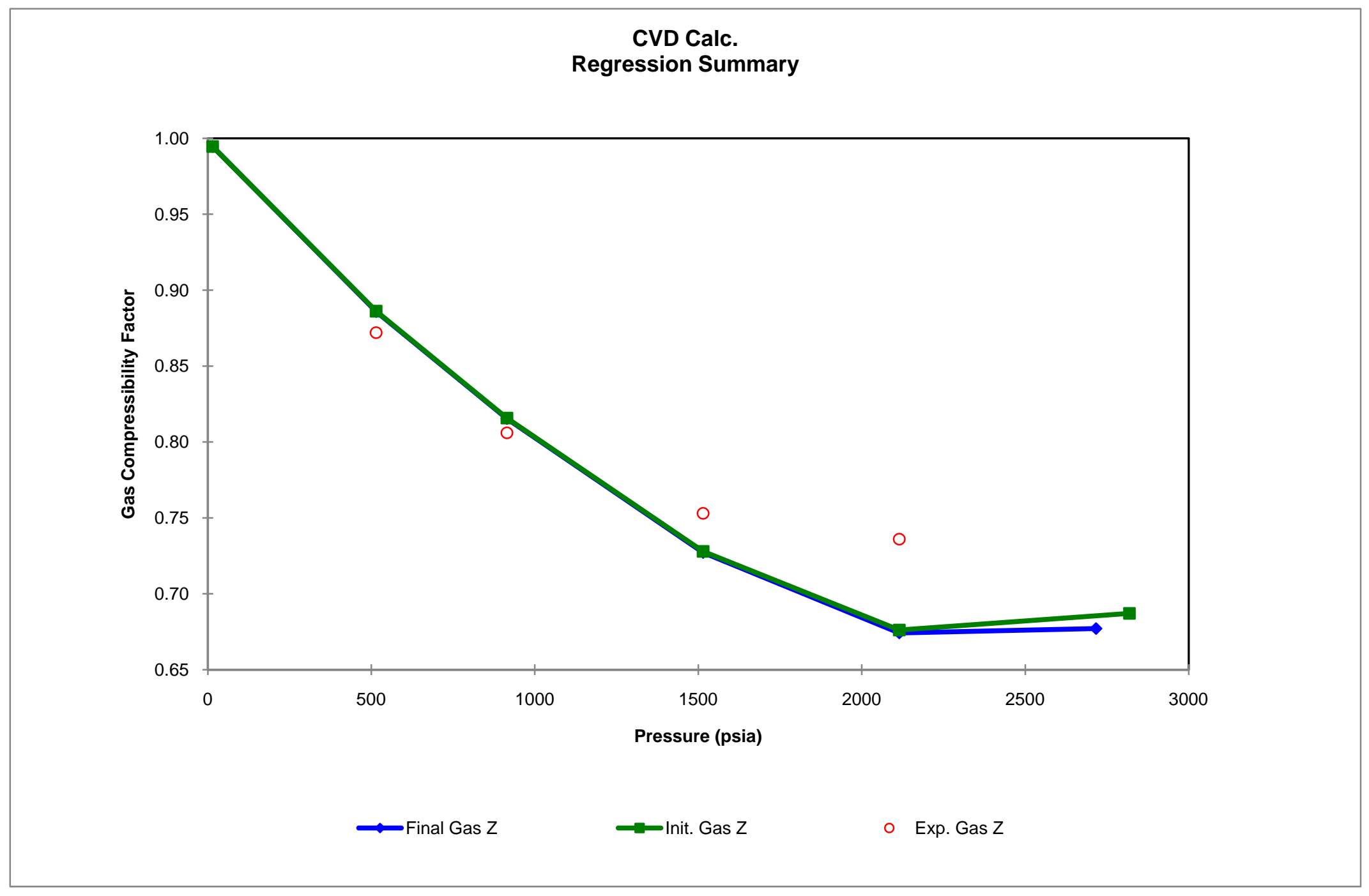




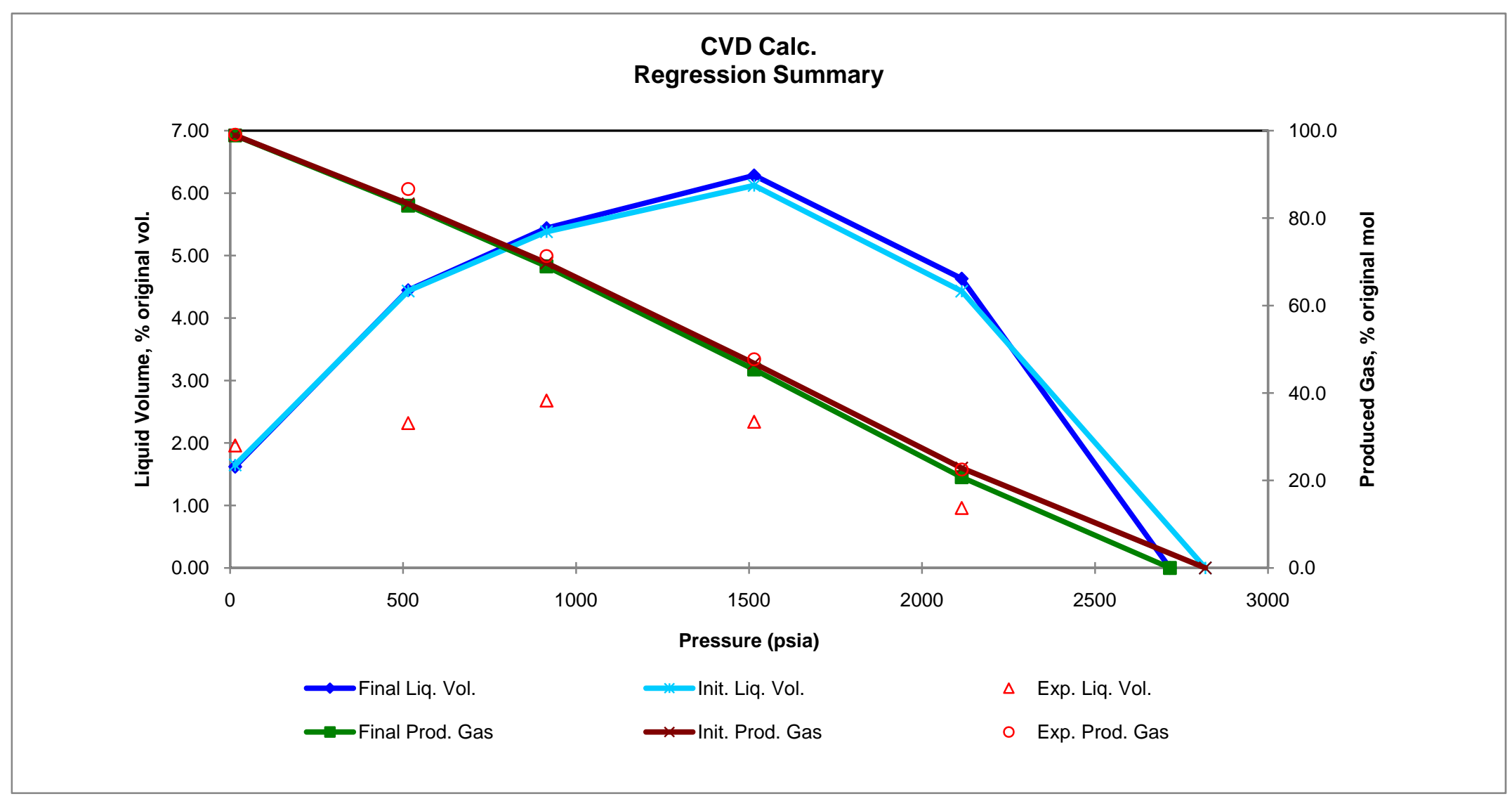




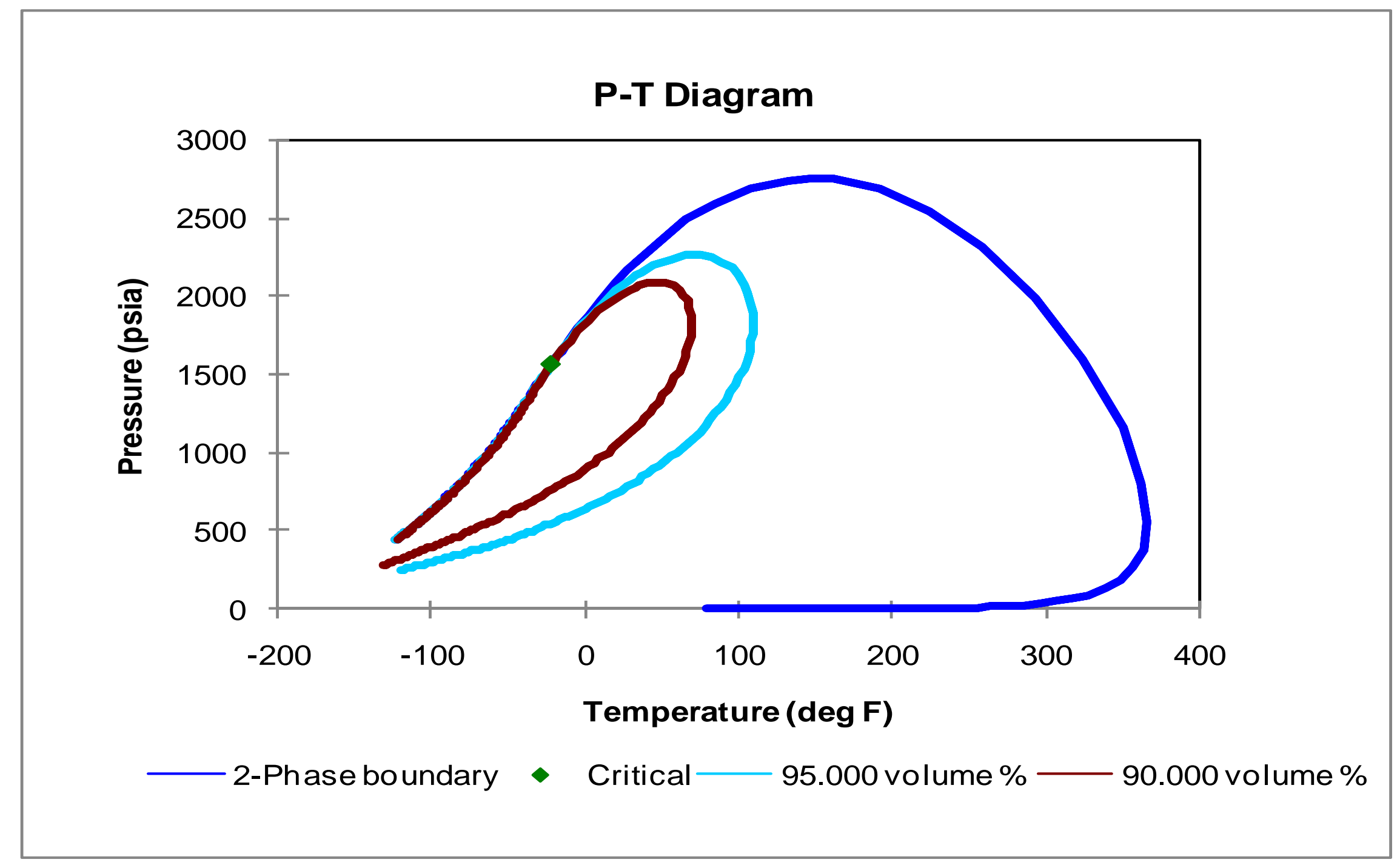




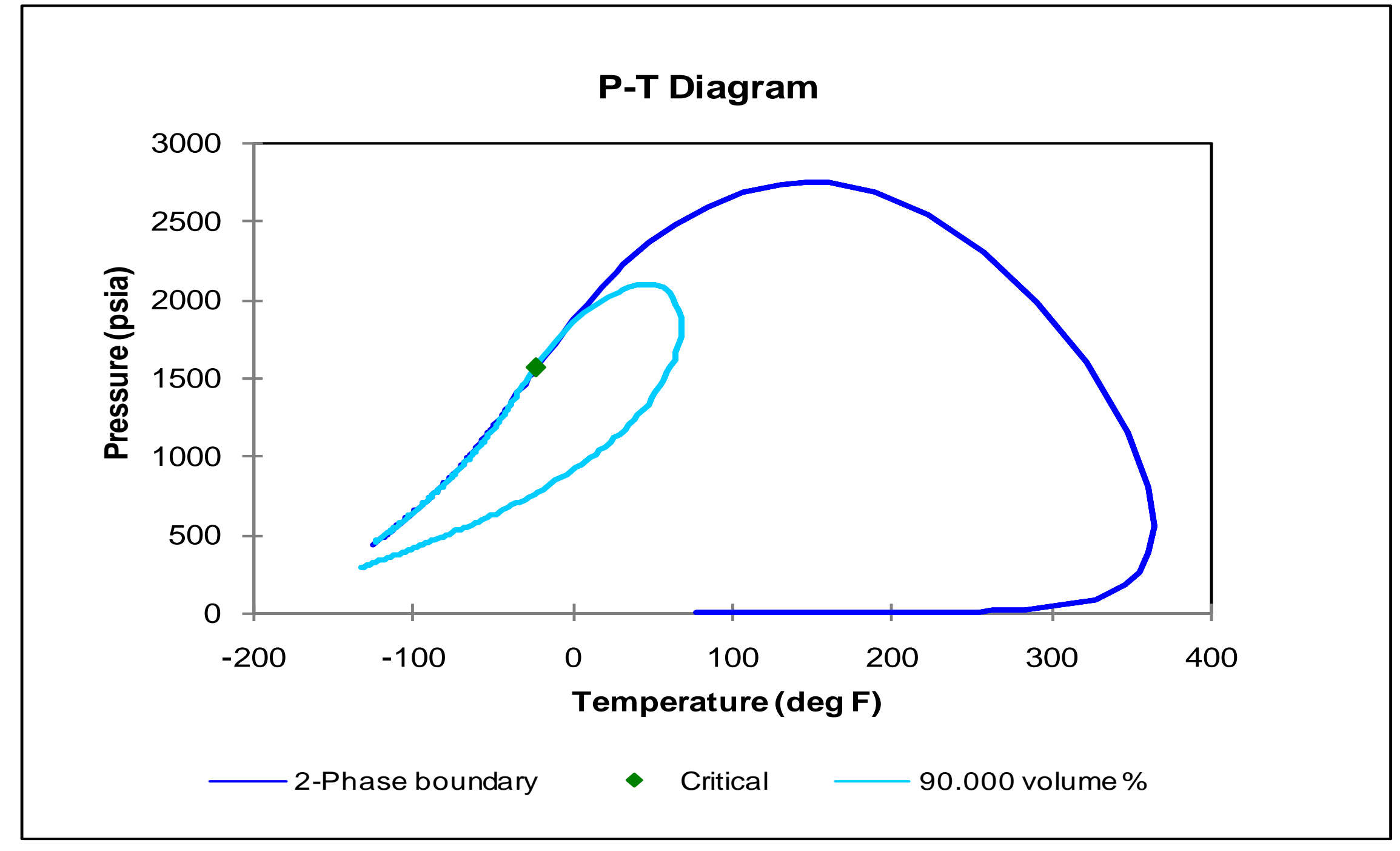

John $\mathrm{H}$.

Hagen 